NTP TECHNICAL REPORT ON THE TOXICOLOGY AND CARCINOGENESIS Studies OF

Dı(2-ethylheXYL) Phthalate (CASRN 117-81-7) ADMINISTERED IN FEED tO SPRAGUe DaWley (HSD:SPRAGUe DAWLEY ${ }^{\circledR}$ SD $^{\circledR}$ ) RATS

NTP TR 601

DECEMBER 2021 


\section{NTP Technical Report on the Toxicology and Carcinogenesis Studies of Di(2-ethylhexyl) Phthalate (CASRN 117-81-7) Administered in Feed to Sprague Dawley (Hsd:Sprague Dawley ${ }^{\circledR}$ SD $^{\circledR}$ ) Rats}

Technical Report 601

December 2021

National Toxicology Program

Public Health Service

U.S. Department of Health and Human Services ISSN: 2378-8925

Research Triangle Park, North Carolina, USA 


\section{Foreword}

The National Toxicology Program (NTP), established in 1978, is an interagency program within the Public Health Service of the U.S. Department of Health and Human Services. Its activities are executed through a partnership of the National Institute for Occupational Safety and Health (part of the Centers for Disease Control and Prevention), the Food and Drug Administration (primarily at the National Center for Toxicological Research), and the National Institute of Environmental Health Sciences (part of the National Institutes of Health), where the program is administratively located. NTP offers a unique venue for the testing, research, and analysis of agents of concern to identify toxic and biological effects, provide information that strengthens the science base, and inform decisions by health regulatory and research agencies to safeguard public health. NTP also works to develop and apply new and improved methods and approaches that advance toxicology and better assess health effects from environmental exposures.

The Technical Report series began in 1976 with carcinogenesis studies conducted by the National Cancer Institute. In 1981, this bioassay program was transferred to NTP. The studies described in the NTP Technical Report series are designed and conducted to characterize and evaluate the toxicological potential, including carcinogenic activity, of selected substances in laboratory animals (usually two species, rats and mice). Substances (e.g., chemicals, physical agents, and mixtures) selected for NTP toxicity and carcinogenicity studies are chosen primarily on the basis of human exposure, level of commercial production, and chemical structure. The interpretive conclusions presented in NTP Technical Reports are derived solely from the results of these NTP studies, and extrapolation of the results to other species, including characterization of hazards and risks to humans, requires analyses beyond the intent of these reports. Selection for study per se is not an indicator of a substance's carcinogenic potential.

NTP conducts its studies in compliance with its laboratory health and safety guidelines and the Food and Drug Administration Good Laboratory Practice Regulations and meets or exceeds all applicable federal, state, and local health and safety regulations. Animal care and use are in accordance with the Public Health Service Policy on Humane Care and Use of Laboratory Animals. Studies are subjected to retrospective quality assurance audits before they are presented for public review. Draft reports undergo external peer review before they are finalized and published.

The NTP Technical Reports are available free of charge on the NTP website and cataloged in PubMed, a free resource developed and maintained by the National Library of Medicine (part of the National Institutes of Health). Data for these studies are included in NTP's Chemical Effects in Biological Systems database.

For questions about the reports and studies, please email NTP or call 984-287-3211. 


\section{Table of Contents}

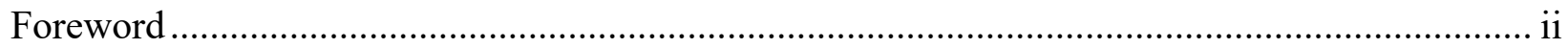

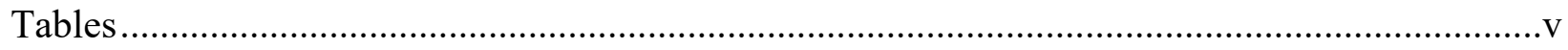

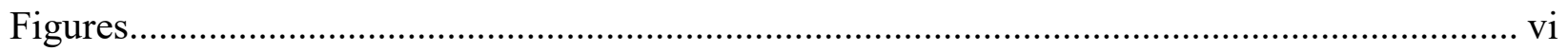

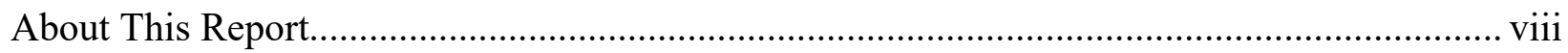

Explanation of Levels of Evidence of Carcinogenic Activity .................................................... xiii

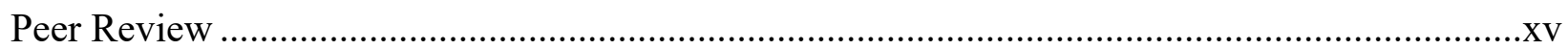

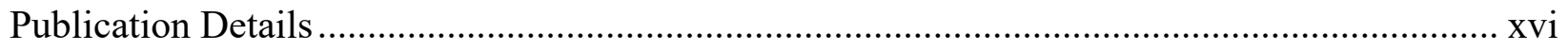

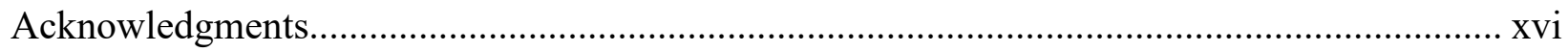

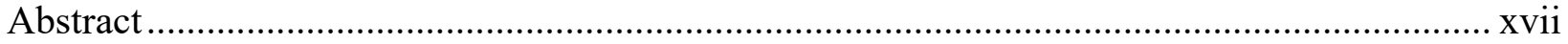

Perinatal and Postweaning Study in Rats (Study 1) ....................................................... xvii

Postweaning-only Study in Rats (Study 2) ..........................................................................

Comparative Carcinogenic Benchmark Dose Analyses ....................................................... xviii

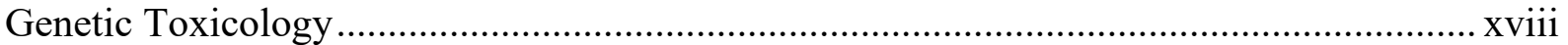

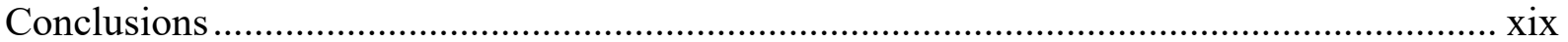

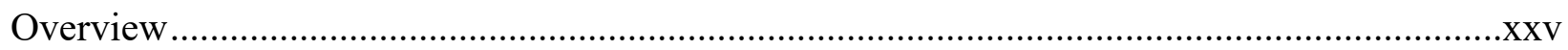

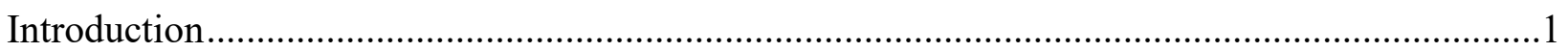

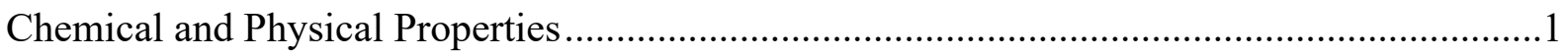

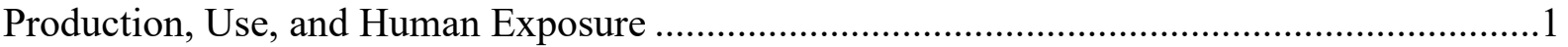

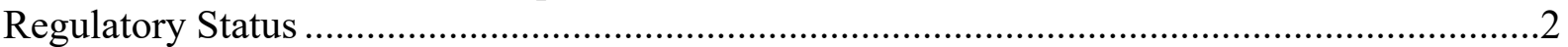

Absorption, Distribution, Metabolism, and Excretion ............................................................ 3

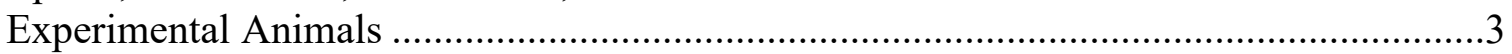

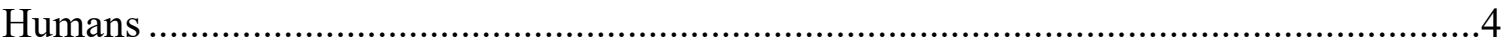

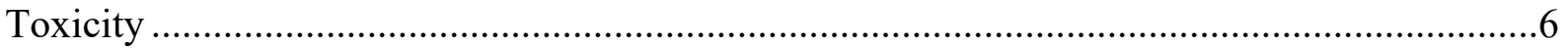

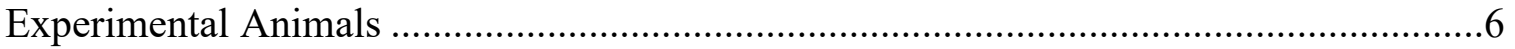

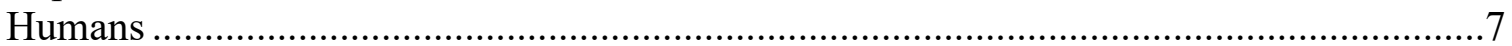

Reproductive and Developmental Toxicity .....................................................................

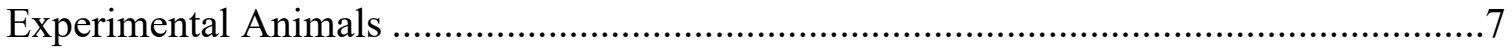

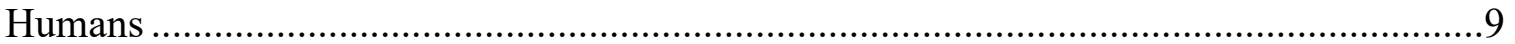

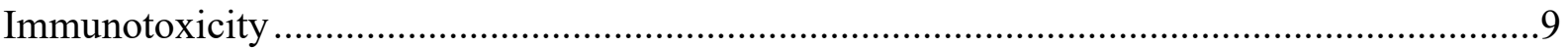

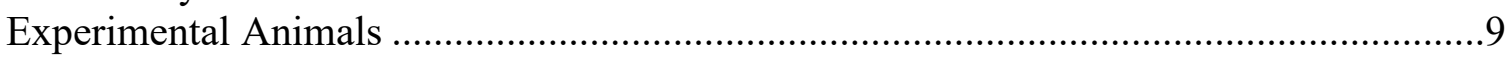

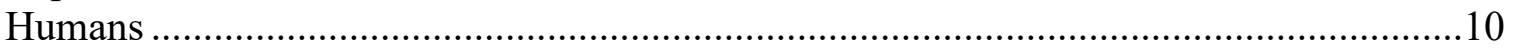

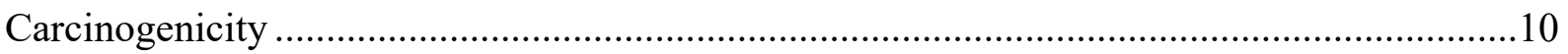

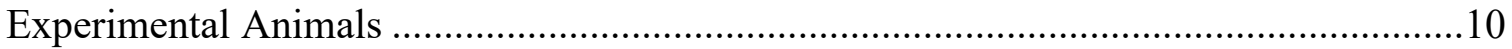

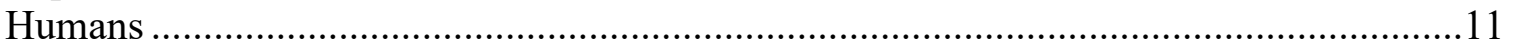

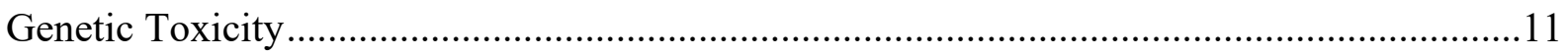

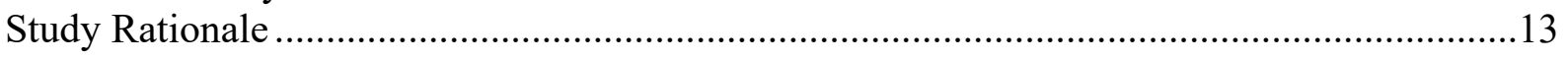

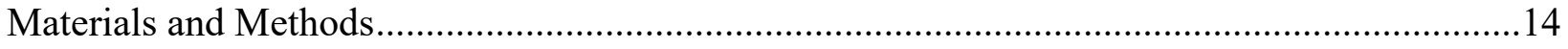

Procurement and Characterization of Di(2-ethylhexyl) Phthalate ............................................14

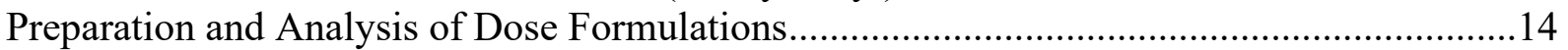


Animal Source

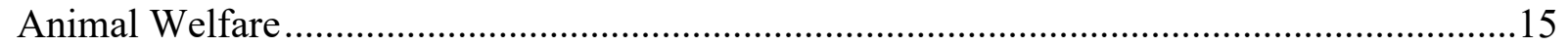

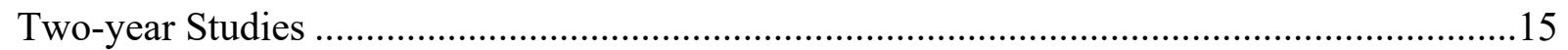

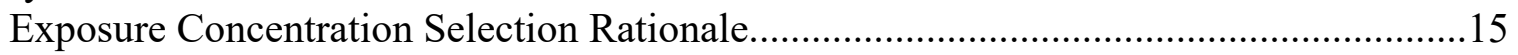

Perinatal and Postweaning Study in Rats (Study 1)......................................................16

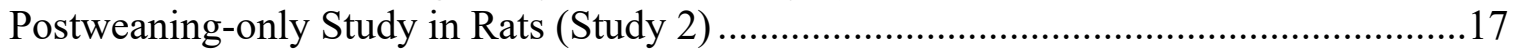

Clinical Examinations and Pathology ………………..............................................17

Benchmark Dose Analysis.....................................................................................21

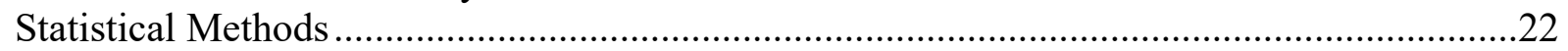

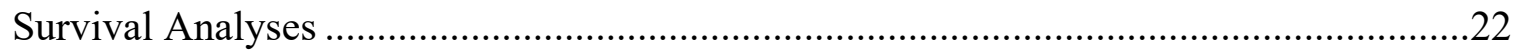

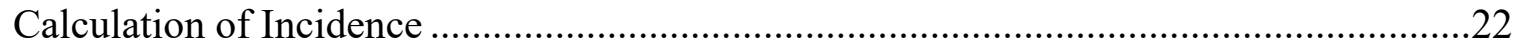

Analysis of Neoplasm and Nonneoplastic Lesion Incidence.............................................22

Analysis of Continuous Variables ..............................................................................24

Analysis of Gestational and Fertility Indices..............................................................24

Body Weight Adjustments..................................................................................24

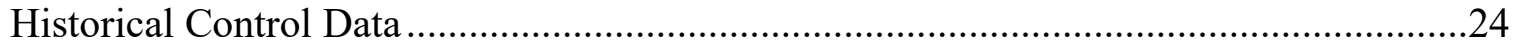

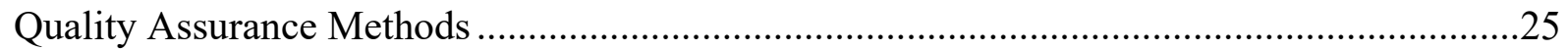

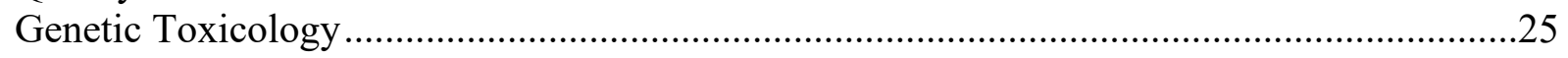

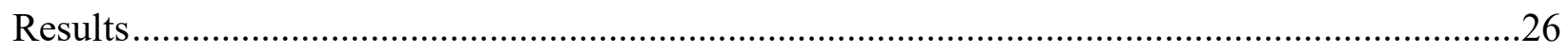

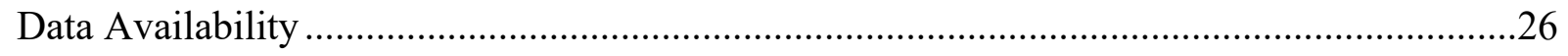

Perinatal and Postweaning Study in Rats (Study 1) ...........................................................26

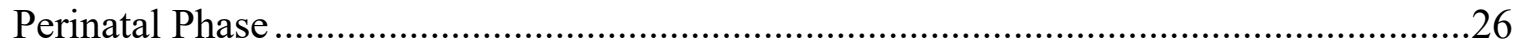

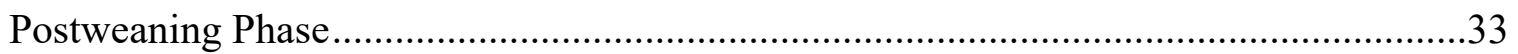

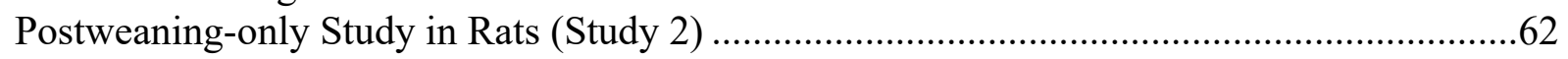

Comparative Carcinogenic Benchmark Dose Analysis ............................................................77

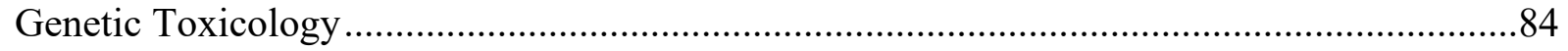

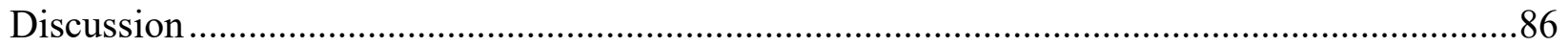

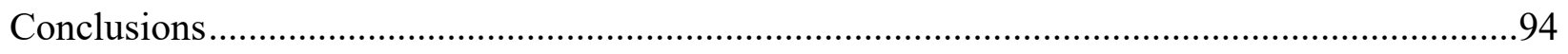

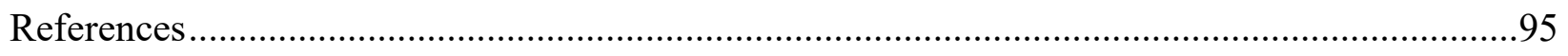

Appendix A. Chemical Characterization and Dose Formulation Studies.................................... A-1

Appendix B. Ingredients, Nutrient Composition, and Contaminant Levels in NIH-07 and

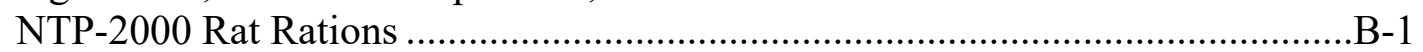

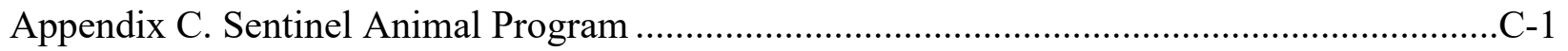

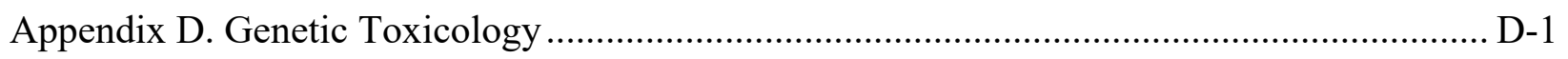

Appendix E. Mono(2-ethylhexyl) Phthalate Internal Dose Assessment …………………...........

Appendix F. Benchmark Dose Analysis ...........................................................................

Appendix G. Peer-review Report.................................................................................... G-1

Appendix H. Supplemental Data ……………............................................................. H-1 


\section{Tables}

Summary of the Two-year Carcinogenesis and Genetic Toxicology Studies of Di(2-ethylhexyl) Phthalate $\mathrm{xx}$

Table 1. Experimental Design and Materials and Methods in the Two-year Feed Studies of Di(2-ethylhexyl) Phthalate.

Table 2. Summary of the Disposition of $F_{0}$ Female Rats during Perinatal Exposure in the Perinatal and Postweaning Two-year Feed Study of Di(2-ethylhexyl) Phthalate...........26

Table 3. Summary of Mean Body Weights and Body Weight Gains of Fo Female Rats during Gestation and Lactation in the Perinatal and Postweaning Two-year Feed Study of Di(2-ethylhexyl) Phthalate

Table 4. Summary of Feed and Di(2-ethylhexyl) Phthalate Consumption by Fo Female Rats during Gestation and Lactation in the Perinatal and Postweaning Two-year Feed Study.....

Table 5. Summary of Mean Litter Size and Survival Ratio of $F_{1}$ Male and Female Rats during Lactation in the Perinatal and Postweaning Two-year Feed Study of Di(2-ethylhexyl) Phthalate

Table 6. Summary of Preweaning $F_{1}$ Male and Female Rat Pup Mean Body Weights Following Perinatal Exposure to Di(2-ethylhexyl) Phthalate

Table 7. Summary of Internal Dose Data for Rats in the Perinatal and Postweaning Twoyear Feed Study of Di(2-ethylhexyl) Phthalate

Table 8. Summary of Survival of Male and Female Rats in the Perinatal and Postweaning Two-year Feed Study of Di(2-ethylhexyl) Phthalate ....

Table 9. Summary of Survival and Mean Body Weights of Male Rats in the Perinatal and Postweaning Two-year Feed Study of Di(2-ethylhexyl) Phthalate.

Table 10. Summary of Survival and Mean Body Weights of Female Rats in the Perinatal and Postweaning Two-year Feed Study of Di(2-ethylhexyl) Phthalate

Table 11. Summary of Feed and Di(2-ethylhexyl) Phthalate Consumption of Male Rats in the Perinatal and Postweaning Two-year Feed Study

Table 12. Summary of Feed and Di(2-ethylhexyl) Phthalate Consumption of Female Rats in the Perinatal and Postweaning Two-year Feed Study....

Table 13. Incidences of Neoplastic and Nonneoplastic Lesions of the Liver in Male and Female Rats in the Perinatal and Postweaning Two-year Feed Study of Di(2ethylhexyl) Phthalate

Table 14. Incidences of Neoplastic and Nonneoplastic Lesions of the Pancreas in Male and Female Rats in the Perinatal and Postweaning Two-year Feed Study of Di(2-ethylhexyl) Phthalate

Table 15. Summary of Gross Lesions in the Reproductive Tract of Male and Female Rats in the Perinatal and Postweaning Two-year Feed Study of Di(2-ethylhexyl) Phthalate

Table 16. Incidences of Neoplastic and Nonneoplastic Lesions of the Testis and Epididymis in Male Rats in the Perinatal and Postweaning Two-year Feed Study of Di(2-ethylhexyl) Phthalate.

Table 17. Incidences of Neoplastic Lesions of the Uterus (Including Cervix) in Female Rats in the Perinatal and Postweaning Two-year Feed Study of Di(2ethylhexyl) Phthalate 
Table 18. Incidences of Nonneoplastic Lesions of the Kidney in Male and Female Rats in the Perinatal and Postweaning Two-year Feed Study of Di(2-ethylhexyl)

Phthalate

Table 19. Incidences of Select Nonneoplastic Lesions of the Heart, Bone Marrow, and Pituitary Gland in Male Rats in the Perinatal and Postweaning Two-year Feed Study of Di(2-ethylhexyl) Phthalate.

Table 20. Summary of Survival of Male and Female Rats in the Postweaning-only Twoyear Feed Study of Di(2-ethylhexyl) Phthalate

Table 21. Summary of Survival and Mean Body Weights of Male Rats in the Postweaning-only Two-year Feed Study of Di(2-ethylhexyl) Phthalate.

Table 22. Summary of Survival and Mean Body Weights of Female Rats in the Postweaning-only Two-year Feed Study of Di(2-ethylhexyl) Phthalate.

Table 23. Summary of Feed and Di(2-ethylhexyl) Phthalate Consumption of Male Rats in the Postweaning-only Two-year Feed Study

Table 24. Summary of Feed and Di(2-ethylhexyl) Phthalate Consumption of Female Rats in the Postweaning-only Two-year Feed Study

Table 25. Incidences of Neoplastic and Nonneoplastic Lesions of the Liver in Male and Female Rats in the Postweaning-only Two-year Feed Study of Di(2-ethylhexyl) Phthalate

Table 26. Incidences of Neoplastic and Nonneoplastic Lesions of the Pancreas in Male and Female Rats in the Postweaning-only Two-year Feed Study of Di(2ethylhexyl) Phthalate

Table 27. Incidences of Neoplastic and Nonneoplastic Lesions of the Testis and Epididymis in Male Rats in the Postweaning-only Two-year Feed Study of Di(2-ethylhexyl) Phthalate

Table 28. Incidences of Neoplastic and Nonneoplastic Lesions of the Uterus (Including Cervix) in Female Rats in the Postweaning-only Two-year Feed Study of Di(2ethylhexyl) Phthalate

Table 29. Incidences of Nonneoplastic Lesions of the Heart, Bone Marrow, and Pituitary Gland in Male Rats in the Postweaning-only Two-year Feed Study of Di(2ethylhexyl) Phthalate

Table 30. Adjusted Incidence Data and Benchmark Dose Modeling for Select Neoplasms in Male Rats in the Two-year Feed Studies of Di(2-Ethylhexyl) Phthalate

Table 31. Adjusted Incidence Data and Benchmark Dose Modeling for Select Neoplasms in Female Rats in the Two-year Feed Studies of Di(2-Ethylhexyl) Phthalate

Table 32. Incidence Data and Benchmark Dose Modeling Results for Testicular Interstitial Cell Adenoma in Male Rats in the Postweaning-only Two-year Feed Study of Di(2-ethylhexyl) Phthalate.

\section{Figures}

Figure 1. Di(2-ethylhexyl) Phthalate (CASRN 117-81-7; Chemical Formula: $\mathrm{C}_{24} \mathrm{H}_{38} \mathrm{O}_{4}$; Molecular Weight: 390.6)

Figure 2. Di(2-ethylhexyl) Phthalate (DEHP) and Metabolites Used to Quantify DEHP Exposure. 
Figure 3. Kaplan-Meier Survival Curves for Rats Exposed to Di(2-ethylhexyl) Phthalate in the Perinatal and Postweaning Two-year Feed Study.

Figure 4. Growth Curves for Rats Exposed to Di(2-ethylhexyl) Phthalate in the Perinatal and Postweaning Two-year Feed Study

Figure 5. Hepatocellular Cytoplasmic Alteration with Pigment in Male Rats Exposed to Di(2-ethylhexyl) Phthalate in the Perinatal and Postweaning Two-year Feed Study (H\&E)

Figure 6. Seminiferous Tubule Dysgenesis in a Male Rat Exposed to Di(2-ethylhexyl)

Phthalate in the Perinatal and Postweaning Two-year Feed Study (H\&E).

Figure 7. Kidney Papilla Edema in Male and Female Rats Exposed to Di(2-ethylhexyl) Phthalate in the Perinatal and Postweaning Two-year Feed Study (H\&E) .56

Figure 8. Renal Papillary Hemorrhage in a Male Rat Exposed to Di(2-ethylhexyl) Phthalate in the Perinatal and Postweaning Two-year Feed Study (H\&E)

Figure 9. Renal Papillary Epithelium Hyperplasia in a Male Rat Exposed to Di(2Ethylhexyl) Phthalate in the Perinatal and Postweaning Two-year Feed Study (H\&E).....

Figure 10. Heart Valve Fibrosis in a Male Rat Exposed to Di(2-Ethylhexyl) Phthalate in the Perinatal and Postweaning Two-year Feed Study (H\&E)...

Figure 11. Pituitary Pars Distalis Hypertrophy in Male Rats Exposed to Di(2-ethylhexyl) Phthalate in the Perinatal and Postweaning Two-year Feed Study (H\&E)

Figure 12. Kaplan-Meier Survival Curves for Rats Exposed to Di(2-ethylhexyl) Phthalate in the Postweaning-only Two-year Feed Study

Figure 13. Growth Curves for Rats Exposed to Di(2-ethylhexyl) Phthalate in the Postweaning-only Two-year Feed Study

Figure 14. Benchmark Dose Modeling Results for Hepatocellular Adenoma or Carcinoma (Combined) in Male Rats

Figure 15. Benchmark Dose Modeling Results for Hepatocellular Adenoma or Carcinoma (Combined) in Female Rats

Figure 16. Benchmark Dose Modeling Results for Pancreatic Acinar Adenoma or Carcinoma (Combined) in Male Rats

Figure 17. Benchmark Dose Modeling Results for Uterine Adenocarcinoma, Adenoma, Squamous Cell Carcinoma, or Squamous Cell Papilloma (Combined) in Female Rats 


\section{About This Report}

National Toxicology Program ${ }^{1}$

${ }^{1}$ Division of the National Toxicology Program, National Institute of Environmental Health

Sciences, Research Triangle Park, North Carolina, USA

\section{Collaborators}

C.R. Blystone, T.D. Hubbard, S.A. Elmore, J.C. Blake, P. Brown, B.L. Burback, M.F. Cesta, D.A. Contos, S.D. Cooper, M.C. Cora, T.A. Crabbs, H.C. Cunny, R.A. Fernando, J.M. Fostel, P.M. Foster, J. Gilliam, H. Gong, C.A. Granville, M. Gruebbel, M.R. Hejtmancik, M.J. Hooth, A.P. King-Herbert, J. Lieause, D.E. Malarkey, C. Martini, B.S. McIntyre, C. Myers, F.M. Parham, G.K. Roberts, V.G. Robinson, N. Sayers, M. Shaw, K.A. Shipkowski, K.R. Shockley, M. Silinski, N.L. South, M.D. Stout, G.S. Travlos, D.Y. Vasconcelos, S. Waidyanatha, N.J. Walker, A.T.D. Watson, R. Whittlesey, G.A. Willson, K.L. Witt

Division of the National Toxicology Program, National Institute of Environmental Health Sciences, Research Triangle Park, North Carolina, USA

Designed studies, evaluated and interpreted results, and reported findings

C.R. Blystone, Ph.D., Co-Study Scientist

T.D. Hubbard, Ph.D., Co-Study Scientist

S.A. Elmore, D.V.M., M.S., Study Pathologist

M.F. Cesta, D.V.M., Ph.D.

M.C. Cora, D.V.M.

H.C. Cunny, Ph.D.

P.M. Foster, Ph.D. (Retired)

M.J. Hooth, Ph.D.

A.P. King-Herbert, D.V.M.

D.E. Malarkey, D.V.M., Ph.D. (Retired)

B.S. McIntyre, Ph.D.

F.M. Parham, Ph.D.

G.K. Roberts, Ph.D.

V.G. Robinson, M.S.

K.A. Shipkowski, Ph.D.

K.R. Shockley, Ph.D.

M.D. Stout, Ph.D.

G.S. Travlos, D.V.M.

S. Waidyanatha, Ph.D.

N.J. Walker, Ph.D.

A.T.D. Watson, Ph.D.

K.L. Witt, M.S.

Provided oversight for data management

J.M. Fostel, Ph.D. 


\section{Battelle, Columbus, Ohio, USA}

Conducted studies and evaluated pathology findings

M.R. Hejtmancik, Ph.D., Principal Investigator

C.A. Granville, Ph.D.

D.Y. Vasconcelos, D.V.M., Ph.D.

Conducted prestart chemistry activities and dose formulations

B.L. Burback, Ph.D.

D.A. Contos, M.S.

N.L. South, B.S.

\section{RTI International, Research Triangle Park, North Carolina, USA}

Conducted preliminary chemistry activities, dose formulations, and biological sample chemistry analyses

R.A. Fernando, Ph.D., Principal Investigator

J.C. Blake, B.A., Deputy Principal Investigator

S.D. Cooper, M.S.

J. Gilliam, B.S.

J. Lieause, M.S.

M. Silinski, Ph.D.

Experimental Pathology Laboratories, Inc., Research Triangle Park, North Carolina, USA Provided pathology review

T.A. Crabbs, D.V.M.

M. Gruebbel, D.V.M., Ph.D.

G.A. Willson, B.V.M.S.

Coordinated NTP Pathology Working Groups on perinatal and postweaning rats (Study 1)

(May 10, 2018) and on postweaning-only rats (Study 2) (September 29, 2016)

T.A. Crabbs, D.V.M. (Study 1)

G.A. Willson, B.V.M.S. (Study 2)

Coordinated NTP Pathology Peer Review on brain glial cell proliferative lesions in rats (Study 1 and Study 2) (April 18, 2016)

M. Gruebbel, D.V.M., Ph.D.

Coordinated NTP Pathology Peer Review on brain granular cell proliferative lesions and head/neck schwannomas/sarcomas in rats (Study 1 and Study 2) (April 18, 2016)

M. Gruebbel, D.V.M., Ph.D.
ASRC Federal,
Prepared data
P. Brown, B.S.
H. Gong, M.S.
C. Martini, B.S.
C. Myers, M.S.
N. Sayers, B.S. 
M. Shaw, B.S.

R. Whittlesey, M.S.

\section{Contributors}

Division of the National Toxicology Program, National Institute of Environmental Health Sciences, Research Triangle Park, North Carolina, USA

Provided oversight for external peer review

S.L. Scruggs, Ph.D.

M.S. Wolfe, Ph.D.

Kelly Government Services, Research Triangle Park, North Carolina, USA

Supported external peer review

E.A. Maull, Ph.D. (retired from NIEHS, Research Triangle Park, North Carolina, USA)

NTP Pathology Working Group, National Institute of Environmental Health Sciences, Research Triangle Park, North Carolina, USA

Participated in NTP Pathology Working Group on perinatal and postweaning rats (Study 1) and postweaning-only rats (Study 2) (August 14, 2018)

N. Barlow, D.V.M., Ph.D., Seattle Genetics, Inc.

M.F. Cesta, D.V.M., Ph.D., National Institute of Environmental Health Sciences

S.A. Elmore, D.V.M., M.S., National Institute of Environmental Health Sciences

R.A. Herbert, D.V.M., Ph.D., National Institute of Environmental Health Sciences

R.C. Kovi, M.V.Sc., Ph.D., Experimental Pathology Laboratories, Inc.

D.E. Malarkey, D.V.M., Ph.D., National Institute of Environmental Health Sciences

C.J. Willson, D.V.M., Ph.D., Integrated Laboratory Systems, LLC

NTP Pathology Peer Review, National Institute of Environmental Health Sciences, Research Triangle Park, North Carolina, USA

Participated in NTP Pathology Peer Review on control rat hearts (Study 1 and Study 2)

(April 14, 2016)

M.F. Cesta, D.V.M., Ph.D., National Institute of Environmental Health Sciences

S.A. Elmore, D.V.M., M.S., National Institute of Environmental Health Sciences

M.P. Jokinen, D.V.M., Integrated Laboratory Systems, LLC

D.E. Malarkey, D.V.M., Ph.D., National Institute of Environmental Health Sciences

Participated in NTP Pathology Peer Review on brain glial cell proliferative lesions (Study 1 and

Study 2) (April 18, 2016)

M.F. Cesta, D.V.M., Ph.D., National Institute of Environmental Health Sciences

P. Little, D.V.M., Ph.D., Experimental Pathology Laboratories, Inc.

D.E. Malarkey, D.V.M., Ph.D., National Institute of Environmental Health Sciences

Participated in NTP Pathology Peer Review on brain granular cell proliferative lesions and head/neck schwannomas and sarcomas (Study 1 and Study 2) (April 18, 2016)

M.F. Cesta, D.V.M., Ph.D., National Institute of Environmental Health Sciences

P. Little, D.V.M., Ph.D., Experimental Pathology Laboratories, Inc.

D.E. Malarkey, D.V.M., Ph.D., National Institute of Environmental Health Sciences 
Performed special review of testis lesions (Study 1) (September 5, 2018)

K.Y. Cimon, D.V.M., Experimental Pathology Laboratories, Inc.

Experimental Pathology Laboratories, Inc., Research Triangle Park, North Carolina, USA Supervised pathology review

T.J. Steinbach, D.V.M., Principal Investigator

G.A. Willson, B.V.M.S., Principal Investigator

Integrated Laboratory Systems, LLC, Research Triangle Park, North Carolina, USA

Conducted micronucleus assays

R.R. Tice, Ph.D., Principal Investigator

Brookhaven National Laboratory, Upton, New York, USA

Conducted chromosomal aberration and micronucleus assays

R.R. Tice, Ph.D., Principal Investigator

CSS Corporation, Research Triangle Park, North Carolina, USA

Prepared quality assessment audits

S. Brecher, Ph.D., Principal Investigator

S. Iyer, B.S.

V.S. Tharakan, D.V.M.

Social \& Scientific Systems, a DLH Company, Research Triangle Park, North Carolina, USA

Provided statistical analyses

S.J. McBride, Ph.D., Principal Investigator

L.J. Betz, M.S.

S.F. Harris, M.S.

J.D. Krause, Ph.D.

ICF, Fairfax, Virginia, USA

Provided contract oversight

D. Burch, M.E.M., Principal Investigator

J. Cleland, M.E.M.

J.A. Wignall, M.S.P.H.

Prepared and edited report

K.S. Duke, Ph.D.

T. Hamilton, M.S.

B. Ingle, Ph.D.

K. McKinley, M.E.M.

M.E. McVey, Ph.D.

J.I. Powers, M.A.P.

K.E. Setty, Ph.D.

K.A. Shipkowski, Ph.D.

S.J. Snow, Ph.D. 
J.W. Tracy, M.H.S.

Supported external peer review

C.N. Byrd, B.S.

L.M. Green, M.P.H.

M.C. Rooney, B.A.

A.J. Schumacher, B.A. 


\section{Explanation of Levels of Evidence of Carcinogenic Activity}

The National Toxicology Program (NTP) describes the results of individual experiments on a chemical agent and notes the strength of the evidence for conclusions regarding each study. Negative results, in which the study animals do not have a greater incidence of neoplasia than control animals, do not necessarily mean that a chemical is not a carcinogen, in as much as the experiments are conducted under a limited set of conditions. Positive results demonstrate that a chemical is carcinogenic for laboratory animals under the conditions of the study and indicate that exposure to the chemical has the potential for hazard to humans. Other organizations, such as the International Agency for Research on Cancer, assign a strength of evidence for conclusions based on an examination of all available evidence, including animal studies such as those conducted by NTP, epidemiologic studies, and estimates of exposure. Thus, the actual determination of risk to humans from chemicals found to be carcinogenic in laboratory animals requires a wider analysis that extends beyond the purview of these studies.

Five categories of evidence of carcinogenic activity are used in the Technical Report series to summarize the strength of evidence observed in each experiment: two categories for positive results (clear evidence and some evidence); one category for uncertain findings (equivocal evidence); one category for no observable effects (no evidence); and one category for experiments that cannot be evaluated because of major flaws (inadequate study). These categories of interpretative conclusions were first adopted in June 1983 and then revised on March 1986 for use in the Technical Report series to incorporate more specifically the concept of actual weight of evidence of carcinogenic activity. For each separate experiment (male rats, female rats, male mice, female mice), one of the following five categories is selected to describe the findings. These categories refer to the strength of the experimental evidence and not to potency or mechanism.

- Clear evidence of carcinogenic activity is demonstrated by studies that are interpreted as showing a dose-related (i) increase of malignant neoplasms, (ii) increase of a combination of malignant and benign neoplasms, or (iii) marked increase of benign neoplasms if there is an indication from this or other studies of the ability of such tumors to progress to malignancy.

- Some evidence of carcinogenic activity is demonstrated by studies that are interpreted as showing a chemical-related increased incidence of neoplasms (malignant, benign, or combined) in which the strength of the response is less than that required for clear evidence.

- Equivocal evidence of carcinogenic activity is demonstrated by studies that are interpreted as showing a marginal increase of neoplasms that may be chemical related.

- No evidence of carcinogenic activity is demonstrated by studies that are interpreted as showing no chemical-related increases in malignant or benign neoplasms.

- Inadequate study of carcinogenic activity is demonstrated by studies that, because of major qualitative or quantitative limitations, cannot be interpreted as valid for showing either the presence or absence of carcinogenic activity.

For studies showing multiple chemical-related neoplastic effects that if considered individually would be assigned to different levels of evidence categories, the following convention has been 
adopted to convey completely the study results. In a study with clear evidence of carcinogenic activity at some tissue sites, other responses that alone might be deemed some evidence are indicated as "were also related" to chemical exposure. In studies with clear or some evidence of carcinogenic activity, other responses that alone might be termed equivocal evidence are indicated as "may have been" related to chemical exposure.

When a conclusion statement for a particular experiment is selected, consideration must be given to key factors that would extend the actual boundary of an individual category of evidence. Such consideration should allow for incorporation of scientific experience and current understanding of long-term carcinogenesis studies in laboratory animals, especially for those evaluations that may be on the borderline between two adjacent levels. These considerations should include:

- adequacy of the experimental design and conduct;

- occurrence of common versus uncommon neoplasia;

- progression (or lack thereof) from benign to malignant neoplasia as well as from preneoplastic to neoplastic lesions;

- some benign neoplasms have the capacity to regress but others (of the same morphologic type) progress. At present, it is impossible to identify the difference. Therefore, where progression is known to be a possibility, the most prudent course is to assume that benign neoplasms of those types have the potential to become malignant;

- combining benign and malignant tumor incidence known or thought to represent stages of progression in the same organ or tissue;

- latency in tumor induction;

- multiplicity in site-specific neoplasia;

- metastases;

- supporting information from proliferative lesions (hyperplasia) in the same site of neoplasia or other experiments (same lesion in another sex or species);

- presence or absence of dose relationships;

- statistical significance of the observed tumor increase;

- concurrent control tumor incidence as well as the historical control rate and variability for a specific neoplasm;

- survival-adjusted analyses and false positive or false negative concerns;

- structure-activity correlations; and

- in some cases, genetic toxicology. 


\section{Peer Review}

The National Toxicology Program (NTP) convened a virtual external ad hoc panel to peer review the draft NTP Technical Report on the Toxicology and Carcinogenesis Studies of Di(2ethylhexyl) Phthalate (CASRN 117-81-7) Administered in Feed to Sprague Dawley Hsd:Sprague Dawley ${ }^{\circledR} S D^{\circledR}$ ) Rats on April 2, 2021. NTP announced the peer-review meeting in the Federal Register (86 FR. 9947. February 17, 2021). The public could view the proceedings online and opportunities were provided for submission of written and oral public comments. The selection of panel members and conduct of the peer review were in accordance with federal policies and regulations. The panel was charged to:

(1) Review and evaluate the scientific and technical elements of each study and its presentation.

(2) Determine whether each study's experimental design, conduct, and findings support the NTP's conclusions regarding the conditions of each study.

NTP carefully considered the panel's recommendations in finalizing the report. The peer-review report is provided in Appendix G. Other meeting materials are available on the NTP website (https://ntp.niehs.nih.gov/go/meeting).

\section{Peer Reviewers}

\section{Gabriele Ludewig, Ph.D., Chairperson}

Professor, Department of Occupational and Environmental Health, College of Public Health University of Iowa

Iowa City, Iowa, USA

\section{Tracie Bunton, D.V.M., Ph.D.}

Founder

Eicarte LLC: Pharmaceutical Preclinical Toxicology and Pathology Consultancy

Gettysburg, Pennsylvania, USA

Michael Elwell, D.V.M., Ph.D.

Consultant, Toxicologic Pathology

APEX TOXPATH, LLC

Apex, North Carolina, USA

Charles Mahrt, D.V.M., Ph.D.

Flagship Biosciences (Retired)

Broomfield, Colorado, USA

Daniel Spade, Ph.D.

Assistant Professor, Department of Pathology and Laboratory Medicine

Brown University

Providence, Rhode Island, USA

John Pierce Wise, Sr., Ph.D.

Professor, Department of Pharmacology and Toxicology

University of Louisville

Louisville, Kentucky, USA 


\section{Publication Details}

Publisher: National Toxicology Program

Publishing Location: Research Triangle Park, NC

ISSN: $2378-8925$

DOI: https://doi.org/10.22427/NTP-TR-601

Report Series: NTP Technical Report Series

Report Series Number: 601

Official citation: National Toxicology Program (NTP). 2021. NTP technical report on the toxicology and carcinogenesis studies of di(2-ethylhexyl) phthalate (CASRN 117-81-7) administered in feed to Sprague Dawley (Hsd:Sprague Dawley ${ }^{\circledR} \mathrm{SD}^{\circledR}$ ) rats. Research Triangle Park, NC: National Toxicology Program. Technical Report 601.

\section{Acknowledgments}

This work was supported by the Intramural Research Program (ES103316, ES103318, and ES103319) at the National Institute of Environmental Health Sciences, National Institutes of Health and performed for the National Toxicology Program, Public Health Service, U.S. Department of Health and Human Services under contracts HHSN273201800006C, HHSN271201800012I, GS00Q14OADU417 (Order No. HHSN273201600015U), HHSN273201600011C, HHSN273201500006C, HHSN273201500014C, HHSN273200800005C, HHSN273201400022C, HHSN273201300009C, HHSN316201200054W, HHSN273201100003C, N01-ES-1-75412, N01-ES-55541, and N01ES-65554. 


\section{Abstract}

Di(2-ethylhexyl) phthalate (DEHP) is a member of the phthalate ester chemical class that occurs commonly in the environment and to which humans are widely exposed. Lifetime exposure to DEHP is likely to occur, including during the in utero and early postnatal windows of development. To date, no carcinogenicity assessments of DEHP have used a lifetime exposure paradigm that includes the perinatal period (gestation and lactation). The National Toxicology Program (NTP) tested the hypothesis that exposure during the perinatal period would alter the DEHP carcinogenic response quantitatively (more neoplasms) or qualitatively (different neoplasm types).

Two chronic carcinogenicity assessments of DEHP were conducted in which Sprague Dawley (Hsd:Sprague Dawley ${ }^{\circledR} \mathrm{SD}^{\circledR}$ ) rats were exposed to dosed feed containing 0, 300, 1,000, 3,000, or 10,000 ppm DEHP for 2 years using different exposure paradigms. In Study 1, groups of $45 \mathrm{~F}_{0}$ time-mated females were provided dosed feed beginning on gestation day (GD) 6 through lactation. On postnatal day (PND) 21, groups of $50 \mathrm{~F}_{1}$ rats per sex continued on the study and were provided dosed feed containing the same DEHP concentration as their respective dam for 2 years. In Study 2, groups of 50 rats per sex, aged 6 to 7 weeks at study start, were provided dosed feed containing DEHP for 2 years.

\section{Perinatal and Postweaning Study in Rats (Study 1)}

During the perinatal period, lower maternal mean body weight, maternal mean body weight gain, and feed consumption were observed in $\mathrm{F}_{0}$ dams exposed to 10,000 ppm DEHP relative to control animals. Also in that exposure group, litter size and pup weights on PND 1 were significantly decreased compared to the control group. Male and female pup mean body weight gains were significantly decreased in the 10,000 ppm group during lactation and resulted in significantly decreased pup body weights at weaning when compared to the control group. Pup survival was not affected following gestational and lactational DEHP exposure.

Following perinatal and 2 years of postweaning DEHP exposure, survival of exposed male and female rats to study termination was similar to that of control groups; however, there were decreases in mean body weight in the 10,000 ppm group compared to the control group.

Significant increases in the incidences of hepatocellular adenoma, hepatocellular adenoma or carcinoma (combined), pancreatic acinar adenoma, and pancreatic acinar adenoma or carcinoma (combined) were observed in the 3,000 and 10,000 ppm male rats relative to the control group. Higher incidences of hepatocellular carcinomas (10,000 ppm males) and pancreatic acinar carcinomas (3,000 ppm males) were also observed. In female rats, significant increases in the incidences of liver neoplasms occurred in the 3,000 ppm (hepatocellular adenoma and hepatocellular adenoma or carcinoma [combined]) and 10,000 ppm (hepatocellular carcinoma and hepatocellular adenoma or carcinoma [combined]) groups. Occurrences of pancreatic acinar adenomas were observed in the 3,000 and 10,000 ppm female groups, and a trend of higher incidence of uterine adenocarcinomas with increasing exposure was observed given the incidence in the 10,000 ppm group. Nonneoplastic lesions were observed in the liver (male and female), pancreas (female), testis, epididymis, kidney (male and female), heart (male only), bone marrow (male only), and pituitary gland (male only). 


\section{Postweaning-only Study in Rats (Study 2)}

Following 2 years of postweaning DEHP exposure, survival of male and female rats was commensurate with or greater than that of control animals, and lower body weights were observed in the 10,000 ppm group. Notably, the magnitude of decreased weight was smaller in the control animals in Study 2 than in the control animals in Study 1. Significant increases in the incidences of hepatocellular adenoma, carcinoma, and adenoma or carcinoma (combined) were observed in male and female rats exposed to $10,000 \mathrm{ppm}$ DEHP relative to the respective control group. In male rats, significantly increased incidences of pancreatic acinar neoplasms were observed in the 3,000 (adenoma) and 10,000 ppm (adenoma and carcinomas) groups. A trend of increasing incidence of testicular interstitial cell adenoma with increasing exposure was observed in male rats given the incidence observed in the 10,000 ppm DEHP group. In female rats, significantly increased incidences of uterine adenocarcinoma and uterine adenoma, adenocarcinoma, squamous cell carcinoma, or squamous cell papilloma (combined) were observed in the 10,000 ppm group compared to the control group. Occurrences of uterine squamous cell papilloma (including multiple) were observed in the $10,000 \mathrm{ppm}$ group. Nonneoplastic lesions were observed in the liver (male and female), pancreas (male and female), testis, epididymis, uterus, heart (male only), bone marrow (male), and pituitary gland (male only).

\section{Comparative Carcinogenic Benchmark Dose Analyses}

Benchmark dose (BMD) levels corresponding to a 10\% increased risk of carcinogenic response $\left(\mathrm{BMD}_{10}\right)$ were estimated for exposure-related carcinogenic responses that were observed in both studies. Generally, the BMDs between studies were within threefold of each other. The lowest estimated BMD 10 (30.99 mg DEHP/kg body weight/day) corresponded to pancreatic acinar adenoma or carcinoma (combined) in males in the postweaning-only study (Study 2).

\section{Genetic Toxicology}

DEHP was tested in a variety of genotoxicity assays in vitro and in vivo; most results were negative. In vitro, negative results were obtained in the following assays: six independent bacterial mutation assays in Salmonella typhimurium bacterial strains (TA100, TA1535, TA1537, TA97, and TA98) with and without exogenous metabolic activation systems (S9 mix; induced hamster, rat, and mouse liver S9), a single mouse lymphoma gene mutation assay (with and without induced rat liver S9 mix), and three independent chromosomal aberration assays conducted in Chinese hamster ovary (CHO) cells (with and without rat liver S9). In nine in vitro sister chromatid exchange tests conducted in CHO cells with and without S9, DEHP produced positive responses in four tests, equivocal results in three, and negative results in two.

In vivo, no increases in chromosomal aberrations were observed in bone marrow cells of female B6C3F1 mice following exposure to DEHP in dosed feed for 14 days. DEHP produced mixed results in three independent erythrocyte micronucleus assays: equivocal in female B6C3F1 mice exposed to DEHP in dosed feed for 14 days, equivocal in male TgAC (FVB/N) mice and positive in female TgAC $(\mathrm{FVB} / \mathrm{N})$ mice following exposure via dosed feed for 26 weeks, and negative in male and female $\mathrm{TgAC}(\mathrm{FVB} / \mathrm{N})$ mice following a 26-week dermal exposure. DEHP produced negative results in two independent studies that tested for induction of sex-linked recessive lethal mutations in Drosophila melanogaster. 


\section{Conclusions}

Under the conditions of the perinatal and postweaning feed study (Study 1), there was clear evidence of carcinogenic activity of di(2-ethylhexyl) phthalate (DEHP) in male Hsd:Sprague Dawley ${ }^{\circledR} \mathrm{SD}^{\circledR}$ rats based on the increased incidences of hepatocellular adenoma or carcinoma (combined) and acinar adenoma or carcinoma (combined) neoplasms (predominately adenomas) of the pancreas. There was clear evidence of carcinogenic activity of DEHP in female Hsd:Sprague Dawley ${ }^{\circledR} \mathrm{SD}^{\circledR}$ rats based on the increased incidence of hepatocellular adenoma or carcinoma (combined). The occurrence of pancreatic acinar adenoma or carcinoma (combined) was considered to be related to exposure. The occurrence of uterine (including cervix) adenoma, adenocarcinoma, squamous cell carcinoma, or squamous cell papilloma (combined) in female rats may have been related to exposure.

Under the conditions of the postweaning-only feed study (Study 2), there was clear evidence of carcinogenic activity of DEHP in male Hsd:Sprague Dawley ${ }^{\circledR} \mathrm{SD}^{\circledR}$ rats based on the increased incidences of hepatocellular adenoma or carcinoma (combined) and acinar adenoma or carcinoma (combined) neoplasms (predominately adenomas) of the pancreas. The occurrence of testicular interstitial cell adenoma in male rats may have been related to exposure. There was clear evidence of carcinogenic activity of DEHP in female Hsd:Sprague Dawley ${ }^{\circledR} \mathrm{SD}^{\circledR}$ rats based on the increased incidences of hepatocellular adenoma or carcinoma (combined) and uterine (including cervix) adenoma, adenocarcinoma, squamous cell carcinoma, or squamous cell papilloma (combined). The occurrence of pancreatic acinar adenoma or carcinoma (combined) in female rats was considered to be related to exposure.

The BMD analysis shows there was no consistent pattern indicating that perinatal and postweaning exposure was more sensitive compared to postweaning-only exposure and modeled responses were within threefold of each other. However, there was a stronger carcinogenic response in the reproductive organs (uterus and testis) in the postweaning-only exposure study compared to the perinatal and postweaning exposure study.

Perinatal and postweaning exposure to DEHP (Study 1) resulted in increased incidence of nonneoplastic lesions in the liver, kidney, heart (male), pancreas (female), pituitary gland (male), bone marrow (male), testis, and epididymis. In addition, exposure increased gross lesions within the reproductive tract of males and females.

Postweaning exposure to DEHP (Study 2) resulted in increased incidence of nonneoplastic lesions in the liver, pancreas, heart (male), pituitary gland (male), bone marrow (male), testis, epididymis, and uterus.

Synonyms: Bis(2-ethylhexyl)phthalate; dioctyl phthalate; phthalic acid di(2-ethylhexyl) ester; bis(2-ethylhexyl) 1,2-benzenedicarboxylate; 1,2-benzenedicarboxylic acid bis(2-ethylhexyl) ester

Trade names: Platinol DOP; Octoil; Silicol 150; Bisoflex 81; Eviplast 80 
Summary of the Two-year Carcinogenesis and Genetic Toxicology Studies of Di(2-ethylhexyl) Phthalate

\begin{tabular}{|c|c|c|c|c|}
\hline & \multicolumn{2}{|c|}{$\begin{array}{l}\text { Perinatal and Postweaning Study } \\
\text { (Study 1) }\end{array}$} & \multicolumn{2}{|c|}{$\begin{array}{l}\text { Postweaning-only Study } \\
\text { (Study 2) }\end{array}$} \\
\hline & $\begin{array}{c}\text { Male } \\
\text { Sprague Dawley } \\
\text { Rats } \\
\end{array}$ & $\begin{array}{c}\text { Female } \\
\text { Sprague Dawley } \\
\text { Rats }\end{array}$ & $\begin{array}{c}\text { Male } \\
\text { Sprague Dawley } \\
\text { Rats }\end{array}$ & $\begin{array}{c}\text { Female } \\
\text { Sprague Dawley } \\
\text { Rats }\end{array}$ \\
\hline Concentrations in Feed & $\begin{array}{l}0,300,1,000,3,000, \\
\text { or } 10,000 \mathrm{ppm}\end{array}$ & $\begin{array}{l}0,300,1,000,3,000, \\
\text { or } 10,000 \mathrm{ppm}\end{array}$ & $\begin{array}{l}0,300,1,000,3,000, \\
\text { or } 10,000 \mathrm{ppm}\end{array}$ & $\begin{array}{l}0,300,1,000,3,000 \\
\text { or } 10,000 \mathrm{ppm}\end{array}$ \\
\hline Survival Rates & $\begin{array}{l}25 / 50,33 / 49,40 / 50 \\
35 / 50,29 / 50\end{array}$ & $\begin{array}{l}31 / 50,32 / 50,34 / 50 \\
34 / 50,27 / 50\end{array}$ & $\begin{array}{l}32 / 50,35 / 50,39 / 50 \\
35 / 50,42 / 50\end{array}$ & $\begin{array}{l}33 / 50,34 / 50,33 / 50 \\
34 / 50,32 / 50\end{array}$ \\
\hline Body Weights & $\begin{array}{l}10,000 \text { ppm group } \\
29.7 \% \text { less than the } \\
\text { control group }\end{array}$ & $\begin{array}{l}3,000 \text { ppm group } \\
9.9 \% \text { less than the } \\
\text { control group; } \\
10,000 \text { ppm group } \\
31.7 \% \text { less than the } \\
\text { control group }\end{array}$ & $\begin{array}{l}10,000 \text { ppm group } \\
15.6 \% \text { less than the } \\
\text { control group }\end{array}$ & $\begin{array}{l}10,000 \text { ppm group } \\
21.9 \% \text { less than the } \\
\text { control group }\end{array}$ \\
\hline Gross Lesions & 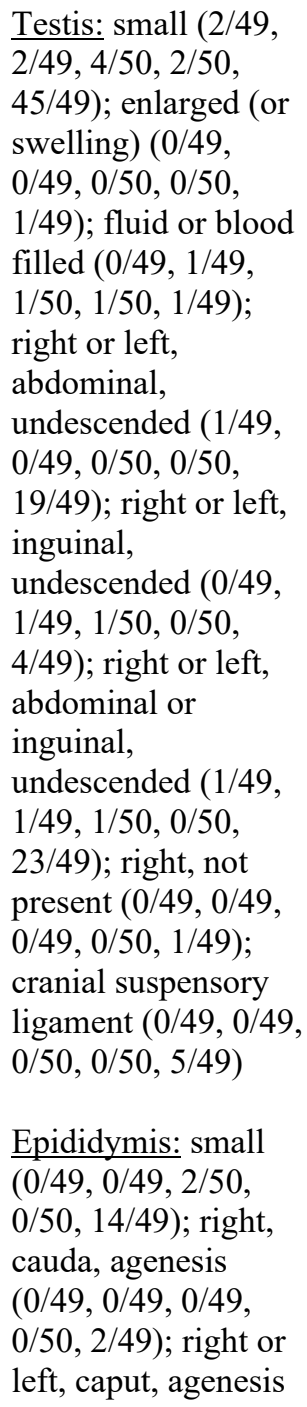 & $\begin{array}{l}\text { Vagina: not patent } \\
(0 / 50,0 / 50,0 / 50 \\
0 / 50,5 / 48) \\
\text { Phallus: cleft }(0 / 50, \\
0 / 50,0 / 50,2 / 50 \\
1 / 48)\end{array}$ & None & None \\
\hline
\end{tabular}




\begin{tabular}{|c|c|c|c|}
\hline \multicolumn{2}{|c|}{$\begin{array}{l}\text { Perinatal and Postweaning Study } \\
\text { (Study 1) }\end{array}$} & \multicolumn{2}{|c|}{$\begin{array}{c}\text { Postweaning-only Study } \\
\text { (Study 2) }\end{array}$} \\
\hline $\begin{array}{c}\text { Male } \\
\text { Sprague Dawley } \\
\text { Rats }\end{array}$ & $\begin{array}{c}\text { Female } \\
\text { Sprague Dawley } \\
\text { Rats }\end{array}$ & $\begin{array}{c}\text { Male } \\
\text { Sprague Dawley } \\
\text { Rats }\end{array}$ & $\begin{array}{c}\text { Female } \\
\text { Sprague Dawley } \\
\text { Rats }\end{array}$ \\
\hline $\begin{array}{l}(0 / 49,0 / 49,0 / 50, \\
0 / 50,4 / 49) ; \text { right or } \\
\text { left, cauda, agenesis } \\
(0 / 49,0 / 49,0 / 50, \\
0 / 50,2 / 49) ; \text { right or } \\
\text { left, corpus, agenesis } \\
(0 / 49,0 / 49,0 / 50 \text {, } \\
0 / 50,3 / 49)\end{array}$ & & & \\
\hline $\begin{array}{l}\frac{\text { Levator }}{\text { ani/bulbocavernosus }} \\
\frac{\text { muscle: small }(0 / 50,}{0 / 49,0 / 50,0 / 50,} \\
2 / 48)\end{array}$ & & & \\
\hline $\begin{array}{l}\text { Cowper's glands: } \\
\text { left, small }(0 / 50, \\
0 / 49,0 / 50,0 / 50, \\
1 / 47) ; \text { right, small } \\
(0 / 50,0 / 49,0 / 50, \\
0 / 50,1 / 47)\end{array}$ & & & \\
\hline $\begin{array}{l}\text { Prostate glands: } \\
\text { small }(0 / 50,0 / 49 \\
0 / 50,0 / 50,1 / 47)\end{array}$ & & & \\
\hline $\begin{array}{l}\frac{\text { Seminal vesicles/ }}{\text { coagulating glands: }} \\
\text { small }(1 / 50,0 / 49, \\
1 / 50,1 / 50,8 / 47)\end{array}$ & & & \\
\hline $\begin{array}{l}\text { Phallus: small }(0 / 50, \\
0 / 49,0 / 49,0 / 50 \\
3 / 49) \text {; cleft }(0 / 50 \\
0 / 49,0 / 49,0 / 50 \\
3 / 49)\end{array}$ & & & \\
\hline $\begin{array}{l}\text { Prepuce: cleft }(0 / 50, \\
0 / 49,0 / 50,0 / 50, \\
1 / 49) ; \text { incomplete } \\
\text { preputial separation } \\
(0 / 50,0 / 49,0 / 50, \\
0 / 50,7 / 49)\end{array}$ & & & \\
\hline $\begin{array}{l}\text { Gubernaculum: right } \\
\text { or left, not present } \\
(0 / 47,0 / 49,0 / 49 \text {, } \\
0 / 50,18 / 41) ; \uparrow \text { right } \\
\text { length }\end{array}$ & & & \\
\hline
\end{tabular}




\begin{tabular}{|c|c|c|c|c|}
\hline & \multicolumn{2}{|c|}{$\begin{array}{l}\text { Perinatal and Postweaning Study } \\
\text { (Study 1) }\end{array}$} & \multicolumn{2}{|c|}{$\begin{array}{l}\text { Postweaning-only Study } \\
\text { (Study 2) }\end{array}$} \\
\hline & $\begin{array}{c}\text { Male } \\
\text { Sprague Dawley } \\
\text { Rats }\end{array}$ & $\begin{array}{c}\text { Female } \\
\text { Sprague Dawley } \\
\text { Rats }\end{array}$ & $\begin{array}{c}\text { Male } \\
\text { Sprague Dawley } \\
\text { Rats }\end{array}$ & $\begin{array}{c}\text { Female } \\
\text { Sprague Dawley } \\
\text { Rats }\end{array}$ \\
\hline Nonneoplastic Effects & $\begin{array}{l}\text { Liver: hepatocyte, } \\
\text { cytoplasmic } \\
\text { alteration }(0 / 50, \\
0 / 49,1 / 50,28 / 50, \\
\text { 37/49); hepatocyte, } \\
\text { hypertrophy }(0 / 50, \\
0 / 49,0 / 50,3 / 50, \\
\text { 17/49); pigment } \\
(0 / 50,1 / 49,5 / 50, \\
\text { 40/50, 38/49); } \\
\text { necrosis }(3 / 50,4 / 49, \\
1 / 50,6 / 50,13 / 49) ; \\
\text { eosinophilic focus } \\
(4 / 50,1 / 49,7 / 50, \\
\text { 2/50,11/49); } \\
\text { basophilic focus } \\
(1 / 50,1 / 49,4 / 50, \\
\text { 4/50,17/49) } \\
\text { Testis: germinal } \\
\text { epithelium, } \\
\text { degeneration } \\
\text { (includes bilateral) } \\
(16 / 49,25 / 49,21 / 50, \\
\text { 21/50, 44/49); } \\
\text { interstitial cell, } \\
\text { hyperplasia, focal } \\
\text { (includes bilateral) } \\
\text { (4/49, 3/49, 6/50, } \\
\text { 5/50, 30/49); } \\
\text { seminiferous tubule, } \\
\text { dysgenesis (includes } \\
\text { bilateral) }(0 / 49,0 / 49, \\
0 / 50,0 / 50,10 / 49) \\
\text { Epididymis: } \\
\text { hypospermia } \\
\text { (includes bilateral) } \\
(4 / 49,5 / 49,12 / 50, \\
8 / 50,43 / 49) \\
\text { Kidney: papilla, } \\
\text { edema (0/50, } 0 / 49, \\
0 / 50,0 / 50,39 / 49) ; \\
\text { papilla, hemorrhage } \\
\text { (0/50, 1/49, 0/50, } \\
\text { 2/50, 12/49); } \\
\text { epithelium, papilla, } \\
\text { hyperplasia }(9 / 50,\end{array}$ & 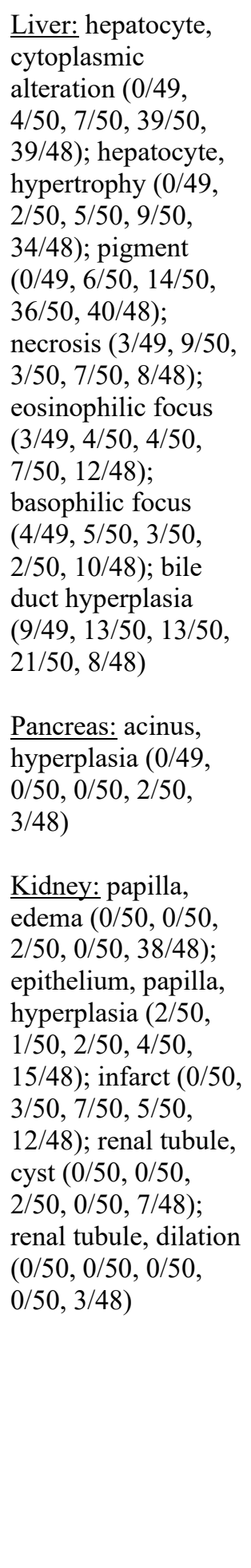 & 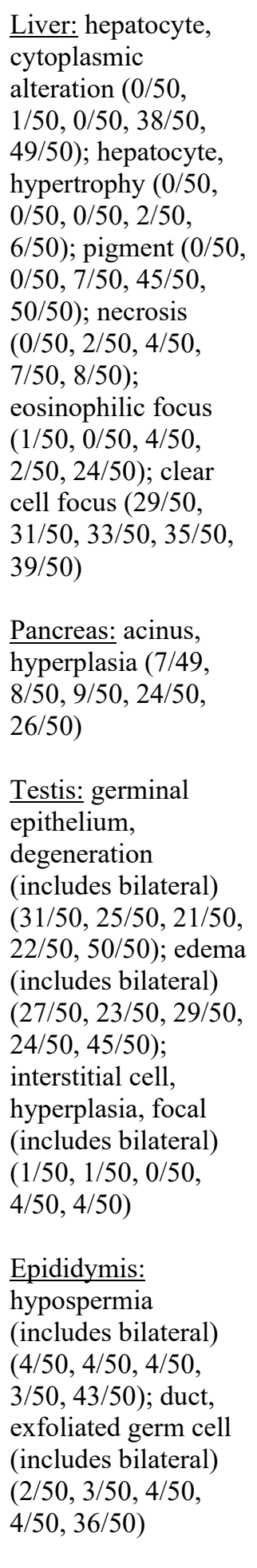 & $\begin{array}{l}\text { Liver: hepatocyte, } \\
\text { cytoplasmic } \\
\text { alteration }(0 / 50, \\
\text { 2/50,15/50,38/50, } \\
\text { 45/49); hepatocyte, } \\
\text { hypertrophy }(0 / 50, \\
\text { 0/50, 6/50, 14/50, } \\
\text { 28/49); pigment } \\
(3 / 50,0 / 50,18 / 50, \\
\text { 30/50, 48/49) } \\
\text { Pancreas: acinus, } \\
\text { hyperplasia }(0 / 50, \\
\text { 1/50, 1/50, 1/50, } \\
\text { 5/47) } \\
\text { Uterus: } \\
\text { inflammation, } \\
\text { chronic }(2 / 50,9 / 50, \\
6 / 50,8 / 50,8 / 49)\end{array}$ \\
\hline
\end{tabular}




\begin{tabular}{|c|c|c|c|c|}
\hline & \multicolumn{2}{|c|}{$\begin{array}{l}\text { Perinatal and Postweaning Study } \\
\text { (Study 1) }\end{array}$} & \multicolumn{2}{|c|}{$\begin{array}{l}\text { Postweaning-only Study } \\
\text { (Study 2) }\end{array}$} \\
\hline & $\begin{array}{c}\text { Male } \\
\text { Sprague Dawley } \\
\text { Rats }\end{array}$ & $\begin{array}{c}\text { Female } \\
\text { Sprague Dawley } \\
\text { Rats }\end{array}$ & $\begin{array}{c}\text { Male } \\
\text { Sprague Dawley } \\
\text { Rats }\end{array}$ & $\begin{array}{c}\text { Female } \\
\text { Sprague Dawley } \\
\text { Rats }\end{array}$ \\
\hline & $\begin{array}{l}\text { 4/49, 4/50, 3/50, } \\
17 / 49) \text {; infarct }(2 / 50, \\
10 / 49,9 / 50,7 / 50, \\
17 / 49) \\
\text { Heart: valve, fibrosis } \\
(0 / 50,2 / 49,1 / 50, \\
3 / 50,11 / 49) ; \text { valve, } \\
\text { thrombus }(0 / 50 \text {, } \\
0 / 49,0 / 50,0 / 50, \\
6 / 49)\end{array}$ & & $\begin{array}{l}\text { Heart: valve, fibrosis } \\
(2 / 50,0 / 50,0 / 50, \\
1 / 50,9 / 50) ; \text { valve, } \\
\text { thrombus }(0 / 50, \\
0 / 50,0 / 50,2 / 50, \\
6 / 50) \\
\text { Bone marrow: } \\
\text { hypercellularity } \\
(18 / 50,22 / 50,30 / 50, \\
25 / 50,34 / 50)\end{array}$ & \\
\hline & $\begin{array}{l}\text { Bone marrow: } \\
\text { hypercellularity } \\
(21 / 50,17 / 49,29 / 50, \\
34 / 50,36 / 50)\end{array}$ & & $\begin{array}{l}\text { Pituitary gland: pars } \\
\text { distalis, hypertrophy } \\
(8 / 50,10 / 50,11 / 50, \\
14 / 50,37 / 50)\end{array}$ & \\
\hline & $\begin{array}{l}\text { Pituitary gland: pars } \\
\text { distalis, hypertrophy } \\
(3 / 50,7 / 49,5 / 50 \\
15 / 50,37 / 49)\end{array}$ & & & \\
\hline \multirow[t]{2}{*}{ Neoplastic Effects } & $\begin{array}{l}\text { Liver: hepatocellular } \\
\text { adenoma }(0 / 50,1 / 49, \\
0 / 50,3 / 50,8 / 49) ; \\
\text { hepatocellular } \\
\text { carcinoma }(1 / 50, \\
0 / 49,0 / 50,0 / 50, \\
\text { 3/49); hepatocellular } \\
\text { adenoma or } \\
\text { carcinoma } \\
\text { (combined) }(1 / 50 \text {, } \\
1 / 49,0 / 50,3 / 50 \text {, } \\
11 / 49)\end{array}$ & $\begin{array}{l}\text { Liver: hepatocellular } \\
\text { adenoma }(1 / 49,0 / 50, \\
5 / 50,9 / 50,5 / 48) ; \\
\text { hepatocellular } \\
\text { carcinoma }(0 / 49, \\
0 / 50,0 / 50,0 / 50, \\
8 / 48) ; \text { hepatocellular } \\
\text { adenoma or } \\
\text { carcinoma } \\
\text { (combined) }(1 / 49 \text {, } \\
0 / 50,5 / 50,9 / 50 \text {, } \\
13 / 48)\end{array}$ & $\begin{array}{l}\text { Liver: hepatocellular } \\
\text { adenoma }(0 / 50,2 / 50, \\
0 / 50,1 / 50,6 / 50) ; \\
\text { hepatocellular } \\
\text { carcinoma }(0 / 50, \\
0 / 50,0 / 50,0 / 50, \\
6 / 50) \text {, hepatocellular } \\
\text { adenoma or } \\
\text { carcinoma } \\
\text { (combined) }(0 / 50 \text {, } \\
2 / 50,0 / 50,1 / 50 \text {, } \\
12 / 50)\end{array}$ & $\begin{array}{l}\text { Liver: hepatocellular } \\
\text { adenoma }(0 / 50,0 / 50 \text {, } \\
1 / 50,1 / 50,13 / 49) ; \\
\text { hepatocellular } \\
\text { carcinoma }(0 / 50, \\
0 / 50,0 / 50,0 / 50, \\
\text { 2/49); hepatocellular } \\
\text { adenoma or } \\
\text { carcinoma } \\
\text { (combined) }(0 / 50, \\
0 / 50,1 / 50,1 / 50, \\
14 / 49)\end{array}$ \\
\hline & $\begin{array}{l}\text { Pancreas: acinar } \\
\text { adenoma }(10 / 50, \\
7 / 49,8 / 50,36 / 50, \\
\text { 22/49); acinar } \\
\text { carcinoma }(0 / 50, \\
0 / 49,0 / 50,3 / 50, \\
\text { 1/49); acinar } \\
\text { adenoma or } \\
\text { carcinoma } \\
\text { (combined) }(10 / 50, \\
7 / 49,8 / 50,38 / 50, \\
22 / 49)\end{array}$ & $\begin{array}{l}\text { Pancreas: acinar } \\
\text { adenoma or } \\
\text { carcinoma } \\
\text { (combined) }(0 / 49, \\
0 / 50,0 / 50,2 / 50, \\
1 / 48)\end{array}$ & $\begin{array}{l}\text { Pancreas: acinar } \\
\text { adenoma }(1 / 49,4 / 50, \\
5 / 50,23 / 50,30 / 50) ; \\
\text { acinar carcinoma } \\
(0 / 49,1 / 50,0 / 50, \\
1 / 50,5 / 50) ; \text { acinar } \\
\text { adenoma or } \\
\text { carcinoma } \\
\text { (combined) }(1 / 49 \text {, } \\
5 / 50,5 / 50,23 / 50, \\
33 / 50)\end{array}$ & $\begin{array}{l}\text { Pancreas: acinar } \\
\text { adenoma or } \\
\text { carcinoma } \\
\text { (combined) }(0 / 50, \\
0 / 50,0 / 50,1 / 50, \\
2 / 47) \\
\text { Uterus: adenoma, } \\
\text { adenocarcinoma, } \\
\text { squamous cell } \\
\text { carcinoma, or } \\
\text { squamous cell } \\
\text { papilloma } \\
\text { (combined) }(2 / 50,\end{array}$ \\
\hline
\end{tabular}




\begin{tabular}{|c|c|c|c|c|}
\hline & \multicolumn{2}{|c|}{$\begin{array}{l}\text { Perinatal and Postweaning Study } \\
\qquad \text { (Study 1) }\end{array}$} & \multicolumn{2}{|c|}{$\begin{array}{l}\text { Postweaning-only Study } \\
\text { (Study 2) }\end{array}$} \\
\hline & $\begin{array}{c}\text { Male } \\
\text { Sprague Dawley } \\
\text { Rats }\end{array}$ & $\begin{array}{c}\text { Female } \\
\text { Sprague Dawley } \\
\text { Rats }\end{array}$ & $\begin{array}{c}\text { Male } \\
\text { Sprague Dawley } \\
\text { Rats }\end{array}$ & $\begin{array}{c}\text { Female } \\
\text { Sprague Dawley } \\
\text { Rats }\end{array}$ \\
\hline & & & & $\begin{array}{l}4 / 50,1 / 50,6 / 50 \\
13 / 50)\end{array}$ \\
\hline Equivocal Findings & None & $\begin{array}{l}\text { Uterus: adenoma, } \\
\text { adenocarcinoma, } \\
\text { squamous cell } \\
\text { carcinoma, or } \\
\text { squamous cell } \\
\text { papilloma } \\
\text { (combined) }(3 / 50, \\
1 / 50,1 / 50,3 / 50, \\
7 / 50)\end{array}$ & $\begin{array}{l}\text { Testis: interstitial } \\
\text { cell, adenoma }(7 / 50 \text {, } \\
3 / 50,3 / 50,6 / 50, \\
15 / 50)\end{array}$ & None \\
\hline $\begin{array}{l}\text { Level of Evidence of } \\
\text { Carcinogenic Activity }\end{array}$ & Clear evidence & Clear evidence & Clear evidence & Clear evidence \\
\hline \multicolumn{5}{|l|}{ Genetic Toxicology } \\
\hline \multicolumn{5}{|c|}{$\begin{array}{l}\text { Bacterial gene mutations: Negative in Salmonella typhimurium strains TA100, TA1535, TA1537, TA97, and } \\
\text { TA98, with and without S9 }\end{array}$} \\
\hline \multicolumn{5}{|c|}{ Mouse lymphoma L5178Y $t k^{+/-}$cells: Negative with and without S9 } \\
\hline \multicolumn{5}{|c|}{ In vitro $\mathrm{CHO}$ cell chromosomal aberration test: Negative with and without $\mathrm{S} 9$} \\
\hline \multicolumn{5}{|c|}{ In vitro $\mathrm{CHO}$ cell sister chromatid exchange test: } \\
\hline \multicolumn{5}{|c|}{ Without rat liver S9: Positive or equivocal in 7 out of 9 studies } \\
\hline \multicolumn{5}{|c|}{ With rat liver S9: Negative in 9 out of 9 studies } \\
\hline \multicolumn{5}{|c|}{ In vivo chromosome aberration test: Negative in female B6C3F1 mice exposed via dosed feed for 14 days } \\
\hline \multicolumn{5}{|c|}{ In vivo micronucleus test in mice: } \\
\hline \multicolumn{5}{|c|}{ B6C3F1 mice: Equivocal in females exposed via dosed feed for 14 days } \\
\hline \multicolumn{5}{|c|}{$\operatorname{TgAC}(\mathrm{FVB} / \mathrm{N})$ mice: Equivocal in males and positive in females exposed via dosed feed for 26 weeks } \\
\hline \multicolumn{5}{|c|}{ TgAC (FVB/N) mice: Negative in males and females exposed dermally for 26 weeks } \\
\hline \multicolumn{5}{|c|}{ Drosophila melanogaster sex-linked recessive lethal test: } \\
\hline \multicolumn{5}{|c|}{ Adult injection: Negative } \\
\hline Larval feeding: Negati & & & & \\
\hline
\end{tabular}




\section{Overview}

Phthalates are plasticizers used to provide flexibility in products composed of polyvinyl chloride plastic or vinyl chloride resins. Studies have shown that in utero and early life phthalate exposure can result in adverse reproductive, developmental, and potentially carcinogenic effects. The National Toxicology Program therefore initiated a broad-based program of work to provide toxicity data and a cancer hazard assessment for lifetime exposure to environmental phthalates. Data generated from this program are intended to facilitate cumulative and aggregate risk characterization efforts for multiple phthalates, including di(2-ethylhexyl) phthalate (DEHP), di$n$-butyl phthalate, and di-isobutyl phthalate.

Previous carcinogenicity assessments of phthalates did not include perinatal exposure (gestation and lactation), whereas human exposure studies indicate that there is lifetime exposure to some phthalates. Thus, whether developmental exposure would alter lifetime DEHP-associated carcinogenic risk is unknown. Exposure during these critical periods of development and growth might be relevant for the evaluation of lifetime toxicological and carcinogenic risk.

The two 2-year toxicity and carcinogenicity studies described in this technical report were conducted to assess whether perinatal exposure would alter lifetime DEHP-associated carcinogenic risk. Specifically, the goal of the studies was to evaluate whether exposure to DEHP during the perinatal period would influence the pattern, dose response, incidence, or severity of the carcinogenic or noncarcinogenic response in rats relative to chronic exposure that does not include this critical period of development and growth. 


\section{Introduction}<smiles>CCCCCC(CC)COC(=O)c1ccccc1C(=O)OCC(CC)CCCC</smiles>

Figure 1. Di(2-ethylhexyl) Phthalate (CASRN 117-81-7; Chemical Formula: $\mathrm{C}_{24} \mathrm{H}_{38} \mathrm{O}_{4}$; Molecular Weight: 390.6).

Synonyms: Bis(2-ethylhexyl)phthalate; dioctyl phthalate; phthalic acid di(2-ethylhexyl) ester; bis(2-ethylhexyl) 1,2benzenedicarboxylate; 1,2-benzenedicarboxylic acid bis(2-ethylhexyl) ester.

Trade names: Platinol DOP; Octoil; Silicol 150; Bisoflex 81; Eviplast 80.

\section{Chemical and Physical Properties}

Di(2-ethylhexyl) phthalate (DEHP) is the diester of phthalic acid and the branched-chain 2-ethylhexanol. DEHP is a pale-yellow to colorless viscous liquid at room temperature and can have a slight odor. DEHP has a boiling point of $384^{\circ} \mathrm{C}$, a melting point of $-55^{\circ} \mathrm{C}$, and a flash point of $215^{\circ} \mathrm{C}$. $^{1}$ At $25^{\circ} \mathrm{C}$, DEHP has a limited water solubility of approximately $0.3 \mathrm{mg} / \mathrm{L}$ and a vapor pressure ranging from $1.42 \times 10^{-7}$ to $9.75 \times 10^{-6} \mathrm{~mm} \mathrm{Hg} .^{2} \mathrm{DEHP}$ has an estimated $\log \mathrm{K}_{\mathrm{ow}}$ of $7.6^{3}$ and is miscible in organic solvents, such as hexane.

\section{Production, Use, and Human Exposure}

DEHP is a widely used member of the phthalate ester chemical class. Phthalates are employed predominantly as plasticizers to provide flexibility in products composed of polyvinyl chloride (PVC) plastic or vinyl chloride resins. DEHP is produced by the esterification of phthalic anhydride with 2-ethylhexanol in the presence of an acid catalyst, such as sulfuric acid or paratoluenesulfonic acid. ${ }^{4}$ DEHP is considered a high-production volume chemical with an estimated 10 to 50 million pounds produced in the United States in 2015, as reported to the U.S.

Environmental Protection Agency (EPA), ${ }^{5}$ a production level consistent with annual production reports from 1986 to 2014, indicating that DEHP use remained consistent.

Globally, between $90 \%$ and $95 \%$ of DEHP is used as a plasticizer in the manufacture of PVC polymers and corresponding products. ${ }^{6 ;} 7$ DEHP is used in a variety of plastic consumer products, including construction materials, shower curtains, garden hoses, floor tiles, automobile upholstery, and food packaging materials. Plastics may contain 1\% to 40\% DEHP by weight, with materials that exhibit increased softness or flexibility likely containing higher levels of DEHP or other phthalates. DEHP is used in the production of medical devices, such as blood bags, enteral/parenteral nutrition bags, peritoneal dialysis bags, and medical tubing. ${ }^{8 ;}{ }^{9}$ Because DEHP is not covalently bonded to the PVC polymer, potential exists for DEHP to leach into contact media. Migration from PVC storage bags into collected blood, blood products, and other biological products is likely associated with the lipophilic nature of DEHP. 
Exposure to DEHP can occur via numerous pathways, such as contact with DEHP-containing plastic products, consumption of foods packaged in plastics, drinking of well water near waste sites, workplace/indoor inhalation of aerosols or particulates containing DEHP, or exposure during certain medical procedures. ${ }^{4}$ The most common exposure pathway is through ingestion of food contaminated with DEHP, which typically occurs because of contact with plastic packaging materials. Migration efficiency of DEHP into foodstuffs from packaging materials is likely associated with the lipophilic nature of DEHP and the contact surface area with the packing materials. In the United States, average daily DEHP exposure from food is estimated to be $0.3 \mathrm{mg} /$ day with a maximum of $2.0 \mathrm{mg}$ /day per individual. ${ }^{10}$ Higher DEHP concentrations ( $\geq 300 \mu \mathrm{g} / \mathrm{kg}$ ) have been noted in poultry, cooking oil, and cream-based dairy products relative to other assessed foodstuffs. ${ }^{11}$ In water, DEHP exhibits low solubility, suggesting a lower relative contribution of drinking water to estimated total daily exposure. ${ }^{12 ; 13}$ Additionally, the low vapor pressure of DEHP indicates a limited capacity for DEHP to volatilize into the air; however, it can readily adsorb to dust particles that can then be respired or ingested. Fromm et al. measured concentrations of DEHP in indoor air and vacuum cleaner dust samples. ${ }^{14}$ The median indoor air DEHP concentration was $156 \mathrm{ng} / \mathrm{m}^{3}$ (95th percentile, $390 \mathrm{ng} / \mathrm{m}^{3}$ ) and $703.4 \mathrm{mg} / \mathrm{kg}(95 \mathrm{th}$ percentile, $1,542 \mathrm{mg} / \mathrm{kg}$ ) in dust samples.

Measurable urinary DEHP metabolite concentrations from participants in the National Health and Nutrition Examination Survey (NHANES) indicate widespread exposure to DEHP in the U.S. population, but have been declining over the years. ${ }^{15}$ Urinary concentration (50th percentile) of a DEHP metabolite, mono(2-ethylhexyl) phthalate (MEHP) (2015-2016) was $1.24 \mu \mathrm{g} / \mathrm{g}$ of creatinine (95th percentile, $5.93 \mu \mathrm{g} / \mathrm{g}$ of creatinine).${ }^{15}$ Using the NHANES data, researchers estimated a median cumulative DEHP exposure of $0.17 \mu \mathrm{g}$ DEHP $/ \mathrm{kg}$ body weight/day ( $\mu \mathrm{g} / \mathrm{kg} /$ day) (95th percentile, $12.0 \mu \mathrm{g} / \mathrm{kg} /$ day). ${ }^{16}$ Urinary concentrations of DEHP and its metabolites are higher in exposed workers relative to unexposed workers and are detected at higher concentrations in postshift relative to preshift samples. ${ }^{14 ; 17 ;} 18$ High exposures have been documented in workers in countries other than the United States and observed in various industries. ${ }^{19-22}$ Newborns and infants may be at risk for higher DEHP exposure relative to the general population due to differences in metabolic capacity, increased food, water, and air intake per unit body weight, and behaviors such as crawling and mouthing, which can increase exposure to contaminants present in soil, house dust, and consumer products. ${ }^{23}$ Additionally, DEHP and its metabolites have been detected in breast milk and baby formula. Average DEHP exposure in nursing infants has been estimated at between 6 and $24 \mu \mathrm{g} / \mathrm{kg} / \mathrm{day} .{ }^{24 ; 25} \mathrm{Multiple}$ DEHP metabolites have also been measured in human amniotic fluid samples, indicating exposure can occur in utero. ${ }^{26}$

DEHP exposure has been associated with certain medical procedures that use PVC plastic bags and tubing is thought to be much higher than from other anticipated environmental exposures. Parenteral exposure to DEHP can occur in patients undergoing medical procedures, such as intravenous administration of drugs, total parenteral nutrition, transfusion of blood or blood products, cardiopulmonary bypass, and extracorporeal membrane oxygenation. ${ }^{8 ; 27-29}$

\section{Regulatory Status}

Numerous regulatory statutes and guidelines are concerned with DEHP levels in consumer products, allowable environmental levels, and limits of occupational exposure. In the Consumer Product and Safety Improvement Act of 2008, issued by Congress, and in a final ruling by the 
U.S. Consumer Product Safety Commission in 2017, any children's toy or childcare articles are prohibited from containing concentrations of more than $0.1 \%$ of eight designated phthalates, including DEHP. ${ }^{30 ; 31}$ FDA regulates the use of DEHP as an indirect food additive used in foodcontact materials. DEHP can be used in semi-rigid and rigid acrylic plastic materials at levels up to $3 \%$ by weight. ${ }^{32}$ Additionally, DEHP can be a component of cellophane food packaging materials if DEHP levels alone or in combination with other phthalates do not exceed 5\% by weight. ${ }^{33}$ EPA established a maximum contaminant level for DEHP in drinking water at $6 \mu \mathrm{g} / \mathrm{L}$ and an oral reference dose of $0.02 \mathrm{mg} / \mathrm{kg}$ /day on the basis of increased relative liver weights in exposed guinea pigs. ${ }^{34-36}$ Due to the potential for increased exposure via inhalation in occupational settings, the Occupational Safety and Health Administration (OSHA) has set an 8hour time-weighted average permissible exposure limit of $5 \mathrm{mg} / \mathrm{m}^{3}$, which is equivalent to the limits recommended by the American Conference of Governmental Industrial Hygienists and the National Institute for Occupational Safety and Health. ${ }^{37}$ The short-term (15-minute) exposure limit allowable by OSHA is $10 \mathrm{mg} / \mathrm{m}^{3}$. The Agency for Toxic Substances and Disease Registry developed DEHP minimal risk levels of 0.1 and $0.06 \mathrm{mg} / \mathrm{kg} /$ day via an oral exposure route for intermediate and chronic exposure durations, respectively. ${ }^{4}$

\section{Absorption, Distribution, Metabolism, and Excretion}

\section{Experimental Animals}

Numerous studies have evaluated the absorption, distribution, metabolism, and excretion (ADME) properties of DEHP. High levels of hydrolase activity present in the intestinal tract of various mammalian species hydrolyze DEHP to its monoester form, MEHP, and 2-ethylhexanol. Endogenous hydrolytic activity has been shown to vary between species. ${ }^{38-41}$ In general, investigators believed that most of consumed DEHP is efficiently hydrolyzed to its monoester form prior to absorption in the intestinal tract, and that absorption of the diester form is associated with high-exposure levels that exceed the hydrolytic capacity of the intestinal pancreatic lipases. Albro et al. ${ }^{40}$ found no DEHP in the livers of rats after oral administration of DEHP at low doses $(<0.4 \mathrm{~g} / \mathrm{kg})$, but did find detectable levels after administration of higher doses $(>0.5 \mathrm{~g} / \mathrm{kg})$. Comparative studies in which male Sprague Dawley rats were administered DEHP by intraperitoneal injection $(4 \mathrm{~g} / \mathrm{kg})$ or oral gavage $(2 \mathrm{~g} / \mathrm{kg})$ revealed that approximately $80 \%$ of the oral dose undergoes mono-de-esterification compared to only $1 \%$ of the parenteral dose. $^{42}$ Co-administration of a pancreatic lipase inhibitor (S,S,S-tributylphosphorothionate) resulted in a marked inhibition of DEHP intestinal absorption, suggesting MEHP is more readily absorbed than its parent molecule, DEHP.

In adult Wistar rats following a single oral administration of $\left[{ }^{14} \mathrm{C}\right]$-DEHP $(2.9 \mathrm{mg} / \mathrm{kg})$, the dose was excreted primarily in the urine $(42 \%)$ and feces $(57 \%)$ by 7 days postadministration, with an estimated absorbed dose of $50 \%$ from the gastrointestinal tract. ${ }^{43}$ Dermal absorption efficiency of $\left[{ }^{14} \mathrm{C}\right]$-DEHP is limited. Only an estimated $6.5 \%$ of a single $30-40 \mathrm{mg} / \mathrm{kg}$ dose in ethanol was absorbed by 7 days postapplication on exposed skin of male Fischer 344 (F344) rats. ${ }^{44}$ Numerous studies report little retention of radiolabeled DEHP or its metabolites in isolated tissues. ${ }^{38 ; 45 ; 46}$ However, elevated concentrations have been detected in rodent liver, adipose tissue, kidney, bladder, testis, and lungs; these findings may be associated with variables of study design such as the administered dose, route of exposure, or duration of exposure prior to necropsy. ${ }^{47-49} \mathrm{~A}$ comparative study in adult male Sprague Dawley rats, male dogs (beagles), and male miniature 
pigs (Hormel strain) reported differential DEHP excretion profiles following dietary exposure to $50 \mathrm{mg} / \mathrm{kg} /$ day for $21-28$ days before administration of a single dose of $\left[{ }^{14} \mathrm{C}\right]-\mathrm{DEHP}$ $(50 \mathrm{mg} / \mathrm{kg}) .{ }^{46}$ Excretion of radioactivity in urine and feces by 24 hours postadministration was $27 \%$ and $57 \%$ (rats), $12 \%$ and $56 \%$ (dogs), and $37 \%$ and $0.1 \%$ (pigs), respectively; and after 4 days was $37 \%$ and $53 \%$ (rats), $21 \%$ and $75 \%$ (dogs), and $79 \%$ and $26 \%$ (mini-pigs), respectively. Overall elimination of radioactivity was complete by postadministration day 4 in all species and was most rapid in rats, followed by dogs, and least rapid in mini-pigs. Sjöberg et al. ${ }^{50}$ investigated the kinetics of DEHP and MEHP in Sprague Dawley rats following a single oral gavage of $1,000 \mathrm{mg} / \mathrm{kg}$ DEHP. In blood samples collected at 1, 3, 7, 9, 12, 15, 24, and 30 hours after dosing, DEHP was only detectable within the first 7 hours after dosing. The maximal plasma concentration $\left(\mathrm{C}_{\max }\right)$ of MEHP occurred within 1 hour of dosing $\left(\mathrm{C}_{\max }\right.$ of $\left.0.093 \mu \mathrm{g} / \mathrm{mL}\right)$, and a plasma elimination half-life of approximately 2.8-3.9 hours was determined. In another study, plasma $\mathrm{C}_{\max }$ of DEHP $(8.8 \mu \mathrm{g} / \mathrm{mL})$ and MEHP $(63.2 \mu \mathrm{g} / \mathrm{mL}$ plasma $)$ were reached within 6 hours of a single oral administration of DEHP in male Wistar rats $(2.8 \mathrm{~g} / \mathrm{kg}) .{ }^{51} \mathrm{In}$ the same study, daily dosing for a week resulted in no accumulation of DEHP or MEHP in plasma.

Additional studies suggest that differential ADME properties during gestational and juvenile development could increase exposure in these sensitive subgroups. DEHP is able to cross the placental barrier, ${ }^{52 ;} 53$ and maternal transfer of DEHP and its metabolites can occur via lactation. ${ }^{54}$ Increased intestinal tissue surface area relative to body weight and higher relative blood flow to the intestines may contribute to higher absorption rates in neonate/juvenile animals than in adults. ${ }^{55}$

Following hydrolysis of DEHP to MEHP by pancreatic lipases in the intestinal tract, MEHP can be further metabolized through oxidation to additional products and undergo subsequent conjugation with glucuronic acid. Interspecies variation in metabolic competencies can lead to distinct urinary metabolite profiles. Oral gavage of DEHP or MEHP to Sprague Dawley or F344 rats resulted in identification of over 20 distinct urinary metabolites. ${ }^{39}$; 40 Phthalic diacids typically constitute most metabolites identified in rat urine. ${ }^{40}$ Rats differ from other tested species in that they display extensive oxidative metabolism of DEHP, but little capacity to conjugate these metabolites. In mice, exposure to MEHP resulted in detectable concentrations of MEHP and metabolite glucuronide conjugates in urine. ${ }^{56 ; 57}$ Primates generally display reduced pancreatic lipase activity in the intestinal tract compared with rodents, leading to reduced conversion of DEHP to MEHP. ${ }^{58}$ Additionally, primates exhibit a reduced capacity to oxidize DEHP metabolites, but an increased capacity to conjugate (glucuronidate) MEHPmetabolites. ${ }^{58}$ Therefore, primates predominately excrete glucuronides of MEHP and metabolites with hydroxyl side chains that require limited oxidative metabolism. ${ }^{40}$

\section{Humans}

Studies have been conducted investigating DEHP toxicokinetic properties in humans. Similar to laboratory mammals, humans hydrolyze DEHP to MEHP by pancreatic lipases in the lumen of the intestinal tract, generate further oxidative metabolites, and conjugate these metabolites for excretion in urine and feces. In a study by Koch et al., urinary and serum concentrations of DEHP metabolites were determined from a human male volunteer following a single oral dose $(0.64 \mathrm{mg} / \mathrm{kg})$ of deuterium-labeled DEHP. ${ }^{59}$ Peak concentrations of three DEHP metabolites [MEHP, mono(2-ethyl-5-hydroxyhexyl) phthalate (MEHHP), and mono(2-ethyl-5-oxohexyl) phthalate (MEOHP)] were reached in serum after 2 hours and in urine after 4 hours. In serum, 
DEHP metabolites were unconjugated and contained high concentrations of MEHP relative to MEHP oxidation products. Urine samples contained higher concentrations of polar MEHP oxidation products than MEHP. Estimated serum elimination half-lives were $<2$ hours for the three measured DEHP metabolites. In a follow-up study, five DEHP urinary metabolites were identified [MEHP, MEHHP, MEOHP, mono(2-ethyl-5-carboxypentyl) phthalate (MECPP), and mono(2-[carboxymethyl]hexyl) phthalate (MCMHP)] that could be used as biomarkers for more accurate estimations of DEHP exposure (Figure 1). ${ }^{60}$ An additional three oxidative metabolitesmono(2-ethyl-3-carboxypropyl) phthalate (MECPrP), mono-2-(1-oxoethylhexyl) phthalate (MOEHP), and mono(2-ethyl-4-carboxybutyl) phthalate (MECBP) - were reported in human biomonitoring studies (Figure 1). ${ }^{61}$ Most of these metabolites undergo phase 2 metabolism to form glucuronide conjugates. Urinary concentrations of MEHHP and MEOHP have been detected at 10-fold higher concentrations than MEHP in 127 paired human samples, suggesting these metabolites may be more sensitive measures of DEHP exposure in the general population. ${ }^{62}$ Given these findings, DEHP exposure could be significantly underestimated in studies that measure only MEHP concentrations to predict human exposure. Other numerous oxidative metabolites of DEHP have also been proposed. ${ }^{61}$

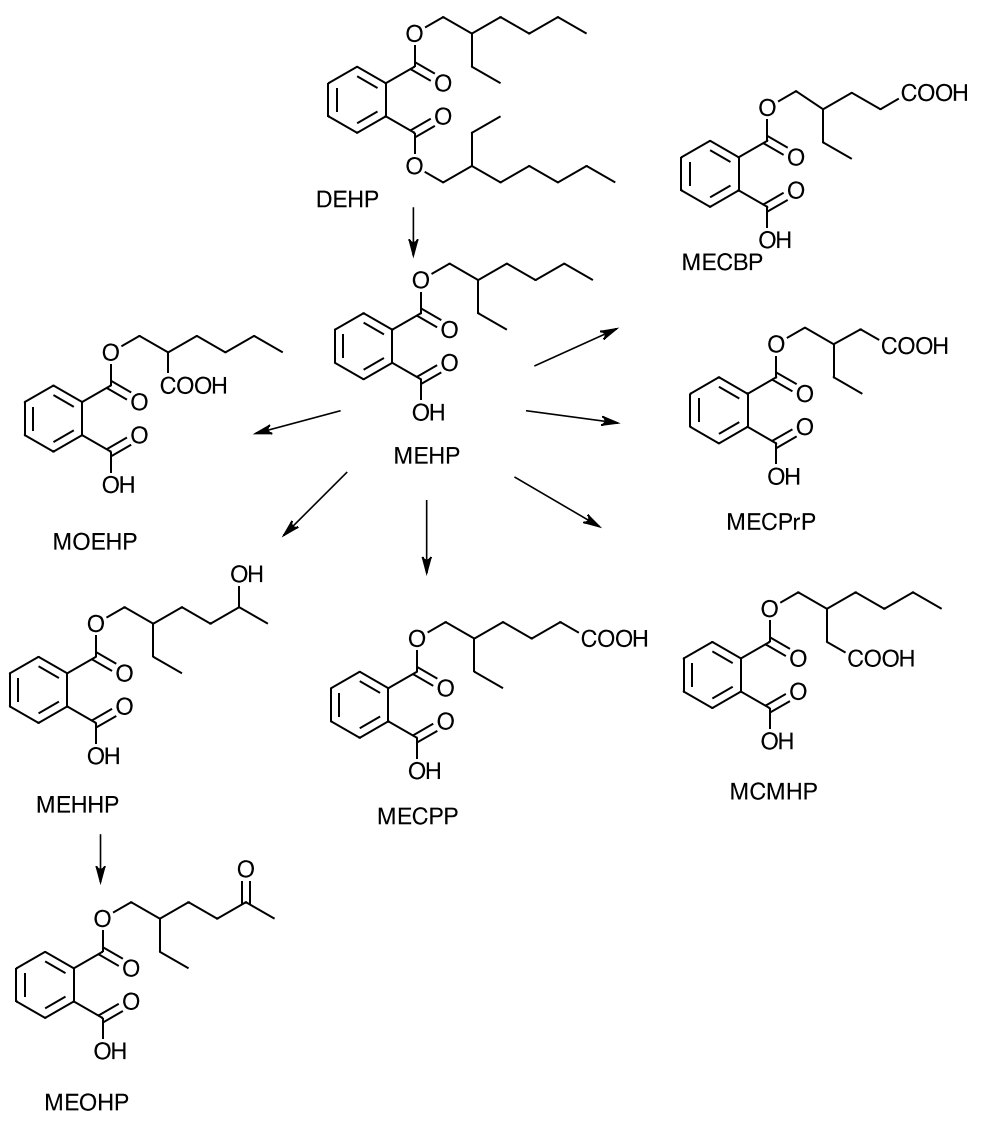

Figure 2. Di(2-ethylhexyl) Phthalate (DEHP) and Metabolites Used to Quantify DEHP Exposure

MCMHP = mono(2-[carboxymethyl]hexyl) phthalate; $\mathrm{MECBP}=\operatorname{mono}(2$-ethyl-4-carboxybutyl $)$ phthalate $\operatorname{MECPP}=\operatorname{mono}(2-$ ethyl-5-carboxypentyl) phthalate; $\mathrm{MECPrP}=\operatorname{mono}(2$-ethyl-3-carboxypropyl) phthalate; $\mathrm{MEHHP}=\operatorname{mono}(2$-ethyl-5hydroxyhexyl) phthalate; MEHP = mono(2-ethylhexyl) phthalate; MEOHP = mono(2-ethyl-5-oxohexyl) phthalate; MOEHP = mono-2-(1-oxoethylhexyl) phthalate. 


\section{Toxicity}

\section{Experimental Animals}

Extensive literature exists on the toxicity of DEHP in numerous animal models. Acute oral median lethal dose (LD50) values for DEHP range from 9,800 to $>40,000 \mathrm{mg} / \mathrm{kg}$ in rats, ${ }^{4 ; 63-65}$ and 9,860 to $>31,360 \mathrm{mg} / \mathrm{kg}$ in mice, ${ }^{4 ; 63 ; 66} \mathrm{LD} 50$ values of $33,900 \mathrm{mg} / \mathrm{kg}$ in rabbits ${ }^{64}$ and $26,300 \mathrm{mg} / \mathrm{kg}$ in guinea pigs have also been reported. ${ }^{63 ;}, 67$ Neonatal and young animals may be more sensitive, however, to the acute effects of DEHP. Mortality was observed in 6- to 21-dayold male Sprague Dawley rats administered five daily oral doses of 1,000 or 2,000 $\mathrm{mg}$ DEHP $/ \mathrm{kg}$, whereas no mortality occurred in rats age 6 weeks or older when administered the same five daily doses. ${ }^{68}$

NTP has reported findings from studies investigating the acute, subchronic, and chronic toxicities of DEHP in rodent models..$^{69}$ No effect on survival was observed in F344 rats or B6C3F1 mice during a 14-day observation period following a single administration of DEHP by oral gavage ( 800 to $20,000 \mathrm{mg} / \mathrm{kg}$ for rats; $1,252 \mathrm{mg} / \mathrm{kg}$ for mice). In 13-week feeding studies, F344 rats were administered a diet containing $0,1,600,3,100,6,300,12,500$, or $25,000 \mathrm{ppm}$ DEHP. Significantly reduced mean body weight gains were observed in male and female rats exposed to $25,000 \mathrm{ppm}$, and testicular atrophy was observed in males exposed to dietary concentrations of $12,500 \mathrm{ppm}$ or higher. B6C3F1 mice exposed to $0,800,1,600,3,100,6,300$, or $12,500 \mathrm{ppm}$ DEHP in the diet for 13 weeks showed similar effects on body weight at the higher concentrations. Decreased mean body weight gains ( $\geq 10 \%$ relative to the control groups) were noted in male mice exposed to 3,100 ppm DEHP or higher and in all DEHP-exposed female mice, except the 1,600 ppm group. In a 2-year study, F344 rats were exposed to $0,6,000$, or $12,000 \mathrm{ppm}$ DEHP in the diet, resulting in mean daily chemical intakes of 322 and $674 \mathrm{mg} / \mathrm{kg}$ body weight ( $\mathrm{mg} / \mathrm{kg}$ ) for males, respectively; and 394 and $774 \mathrm{mg} / \mathrm{kg}$ for females, respectively. At the end of the 2-year study, mean body weights of exposed rats were up to $20 \%$ lower in the high-exposure groups compared to the control groups. In a companion 2-year study, $\mathrm{B} 6 \mathrm{C} 3 \mathrm{~F} 1$ mice were exposed to $0,3,000$, or $6,000 \mathrm{ppm}$ DEHP in the diet, resulting in mean daily chemical intakes of 672 and $1,325 \mathrm{mg} / \mathrm{kg}$ for males, respectively, and 799 and $1,821 \mathrm{mg} / \mathrm{kg}$ for females, respectively. At the end of the 2-year study, mean body weights were $7 \%$ and $10 \%$ lower in the 3,000 and $6,000 \mathrm{mg} / \mathrm{kg}$ male groups, and $21 \%$ and $33 \%$ lower in the 3,000 and $6,000 \mathrm{mg} / \mathrm{kg}$ female groups, respectively, relative to the control groups. The incidence of testicular tubule degeneration or atrophy was significantly elevated in high-exposure group male rats (approximately 90\%) and male mice (approximately 14\%) relative to the control groups.

Numerous laboratory animal studies have reported reductions in body weight and body weight gain following repeated exposures to DEHP, and common target organs of DEHP toxicity include the testis, kidney, and liver. Toxic effects of phthalates on the male reproductive tract are well characterized and are addressed in a subsequent section of this Introduction. DEHP effects on the kidney include reduced creatinine clearance, increased absolute and relative kidney weights, increased incidence and/or severity of mineralization of renal papilla, increased incidence and/or severity of tubule cell pigment, and increased incidence and/or severity of chronic progressive nephropathy ${ }^{70-72}$ Liver enlargement due to both hepatocyte hyperplasia and hypertrophy, with associated morphological changes such as increased size and number of peroxisomes and corresponding increases in fatty-acid metabolism, are known hallmarks of DEHP toxicity in rodents. Activation of the peroxisome proliferator-activated receptor alpha 
$(\mathrm{PPAR} \alpha)$ in hepatocytes is recognized as a key molecular initiating event by which DEHP induces adverse effects in the liver. ${ }^{73}$ PPAR $\alpha$-deficient mice did not exhibit characteristic liver toxicity following 24 weeks of DEHP exposure but did exhibit moderate kidney and testicular toxicity. ${ }^{74}$ These findings suggest that while DEHP-induced liver toxicity is associated with PPAR $\alpha$ status, renal and testicular toxicities likely manifest via alternative mechanisms.

Decreased severity of hepatic effects in nonrodent species may be related to interspecies differences in PPAR $\alpha$ expression, binding, localization, and downstream molecular signaling pathways. ${ }^{41}$ DEHP metabolites such as MEHP have been reported to be more potent activators of human and mouse PPAR $\alpha$ relative to its parent molecule. ${ }^{75}$ Therefore, interspecies differences in pancreatic lipase activity, which converts DEHP to MEHP, may influence observed DEHP toxicities. Additionally, routes of administration that bypass first pass metabolism in the intestinal tract and liver (intravenous), reducing hydrolysis of DEHP to its metabolites, could influence subsequent toxicity.

\section{Humans}

The health effects of DEHP have been evaluated extensively in animal models, but data that address the relationship between human health effects or adverse outcomes and exposure to DEHP are limited. Shaffer et al. ${ }^{64}$ presented a case report in which two male subjects had ingested single DEHP doses of $5 \mathrm{~g}$ and $10 \mathrm{~g}$, respectively. ${ }^{64}$ The individual who consumed the $10 \mathrm{~g}$ dose presented with symptoms of mild gastric disturbance and moderate diarrhea, whereas no effects were observed at the lower dose.

\section{Reproductive and Developmental Toxicity}

\section{Experimental Animals}

Studies with laboratory rodents demonstrate that DEHP exposure can cause adverse effects on reproduction and development. In adult rats, oral DEHP exposure is associated with numerous deleterious effects on the male reproductive tract, including decreased weights of the testes, prostate, seminal vesicles, and epididymis; degeneration and atrophy of the seminiferous tubules; altered sperm parameters; and reduced fertility. $4 ; 13$ The testes are considered a primary target tissue of DEHP toxicity. Decreased testicular weight and increased incidence of tubular atrophy have been observed in numerous rodent studies in which doses exceeded $100 \mathrm{mg} / \mathrm{kg} / \mathrm{day} .{ }^{69 ; 76-82}$ Within the testes, DEHP appears to preferentially target Sertoli cell populations and directly or indirectly Leydig cell populations, which can impair spermatogenesis and fertility. ${ }^{78 ; 83 ; 84}$ Irregular seminiferous tubule structure and altered spermatogenesis were evident in male rats ingesting DEHP at 2,000 mg/kg/day via the diet for 15 days ${ }^{85}$ In these rats, few spermatozoa were present in the lumen of the tubules, and damaged spermatogenic cells were observed in the tubular space. Significantly increased incidences of bilateral aspermatogenesis were observed at lower exposure concentrations $(29 \mathrm{mg} / \mathrm{kg} /$ day) in male rats fed DEHP-supplemented diets for 2 years. ${ }^{86}$ Prepubertal rodents appear to be more sensitive to DEHP-mediated effects on the testes relative to sexually mature rodents. ${ }^{76 ; 82}$ In contrast to studies in rodents, no changes in testes/epididymides weight or testicular histology were observed in cynomolgus monkeys following administration of $500 \mathrm{mg} / \mathrm{kg} /$ day DEHP by gavage for 14 consecutive days. ${ }^{87}$ Decreased fertility also has been observed in female rodents exposed to DEHP, and may be related to DEHP-induced disruption of normal estrous/ovulatory cycles. ${ }^{88}$ 
DEHP is a developmental toxicant in rodents, producing embryotoxic, fetotoxic, and teratogenic effects. Decreased fetal/pup body weight, increased rates of abortion and fetal resorptions, and malformations (hydronephrosis, cardiovascular malformations, and tail malformations) have been reported in rat dams and corresponding litters after exposure to DEHP during pregnancy/gestation. ${ }^{89 ; 90}$ Exposure to DEHP during the perinatal period (gestation and/or lactation) can induce abnormal development of the male reproductive tract and other androgen-sensitive tissues. Although the exact mechanism is unknown, DEHP acts as an endocrine disruptor via an antiandrogenic mode of action and decreases insulin-like hormone 3 production by Leydig cells. Normally, during the window of fetal male sexual differentiation (gestation days 15.5-21.5), androgen-dependent masculinization of the fetal reproductive tract occurs, resulting in differentiation of the internal (epididymis, vas deferens, seminal vesicles, prostate) and external (penis, scrotum, perineum) genitalia. ${ }^{91 ; 92}$ Exposure to DEHP during this critical window of susceptibility decreases fetal testosterone synthesis leading to structural malformations and functional alterations of the male reproductive system. ${ }^{93 ;} 94$ Reduced anogenital distance (AGD), retained nipples, penile morphological abnormalities (hypospadias), undescended testes (cryptorchidism), small/absent sex accessory glands, delays in onset of pubertal landmarks (preputial separation), and histopathological alterations in testes and epididymides have been observed in male rats following perinatal DEHP exposure..$^{93 ; 95-97}$ Dysmorphogenic effects in the testes include microscopic disorganization of the seminiferous tubules with detachment of the spermatogonial cells from the basal membrane and absence of spermatocytes. ${ }^{98}$ The term "phthalate syndrome" is often used to describe the compendium of reproductive tract malformations observed in male test animals following in utero phthalate exposure. $^{99}$

The reproductive and developmental effects of DEHP exposure were comprehensively investigated by NTP in a multigenerational reproductive assessment by continuous breeding study. ${ }^{100}$ In this study, DEHP was administered in the diet at concentrations of 1.5 (control), 10, $30,100,300,1,000,7,500$, or $10,000 \mathrm{ppm}$ to Sprague Dawley rats over multiple successive generations $\left(\mathrm{F}_{0}, \mathrm{~F}_{1}, \mathrm{~F}_{2}\right.$, and $\left.\mathrm{F}_{3}\right)$ throughout the breeding, gestational, lactational, and postweaning intervals. Measured feed consumption and body weights informed the calculation of approximate daily doses of $0.12,0.78,2.4,7.9,23,77,592$, and $775 \mathrm{mg} / \mathrm{kg} /$ day in the $\mathrm{F}_{0}$ animals; $0.09,0.48$, $1.4,4.9,14,48,391$, and $543 \mathrm{mg} / \mathrm{kg} /$ day in the $\mathrm{F}_{1}$ animals; and $0.1,0.47,1.4,4.8,14$, 46, and $359 \mathrm{mg} / \mathrm{kg} /$ day in the $\mathrm{F}_{2}$ animals. The $10,000 \mathrm{ppm}$ group was removed from the study following the $\mathrm{F}_{1}$ generation due to the inability to produce offspring ( $\mathrm{F}_{2}$ generation). Adverse reproductive and developmental effects such as decreased pregnancy index, decreased male AGD, delayed onset of pubertal landmarks (testes descent, vaginal opening, and balanopreputial separation), sperm counts, small male reproductive organs (testes, epididymis, and caudal epididymis), and seminiferous tubule atrophy were observed in all generations in the 7,500 and 10,000 ppm groups. No reproductive toxicity was evident at exposure concentrations $<7,500 \mathrm{ppm}$; however, increased incidences of small testes and prostates were noted in 300 and 1,000 ppm male rats. After further review of animal studies by NTP's Center for the Evaluation of Risks to Human Reproduction (CERHR) expert panel, a developmental no-observed-effect level of 1,000 ppm was suggested and calculated to be no more than $46 \mathrm{mg} / \mathrm{kg} /$ day based on the average dose over three generations. ${ }^{13}$ 


\section{Humans}

Given the results from animal studies, there is significant concern that DEHP can adversely affect human reproduction and male development. FDA's CDRH concluded that "children undergoing certain medical procedures may represent a population at increased risk for the effects of DEHP." A similar conclusion was reached by NTP's CERHR, which found cause for "serious concern" that certain medical treatments may result in DEHP exposure levels that could adversely affect the development of the reproductive tract in male infants. ${ }^{13}$ Numerous epidemiological studies have found no significant association of DEHP or its metabolites with sperm abnormalities, circulating hormone concentrations, or indications of infertility. ${ }^{101-105}$ Other studies have reported associations between maternal urinary DEHP metabolite concentrations and effects on several markers of human male genital development. In complementary studies by Swan et al., measures of AGD and penile width in male infants were significantly associated with exposure to DEHP and three of its metabolites. ${ }^{106 ; 107}$ Many parallels exist between the "phthalate syndrome" suite of effects in animal models and descriptions of human testicular dysgenesis syndrome. This syndrome is characterized by increased incidences of reproductive tract malformations in male newborns (cryptorchidism, hypospadias) and adverse effects in young adults (low sperm counts, testicular germ cell cancer) and is likely related to in utero exposure to environmental chemicals. ${ }^{108 ; 109}$

\section{Immunotoxicity}

\section{Experimental Animals}

Several studies have been conducted to assess the potential of DEHP or MEHP to modulate immune function. Studies by Larsen et al. found subcutaneous injections of MEHP (100 $\mu \mathrm{g})$ to induce an immunosuppressive effect characterized by a reduction in immunoglobulin $\mathrm{E}$ (IgE) and IgG1 antibody production in BALB/cJ mice following co-administration of ovalbumin antigen. ${ }^{110}$ However, in the same study, lower doses of MEHP $(1 \mu \mathrm{g})$ induced an adjuvant effect characterized by increased IgE production. Administration of DEHP to male F344 rats via the $\operatorname{diet}(12 \mathrm{ppm})$ for 21 days resulted in a suppressed hepatic T-helper Type 1 (Th1) immune response initiated via intraperitoneal exposure to Mycobacterium bovis purified protein derivative. ${ }^{111}$ The authors hypothesized that this effect was associated with biotransformation of DEHP to MEHP and subsequent activation of PPAR $\alpha$. Further studies investigating the mixed Thelper cell adjuvant properties of DEHP found that this effect occurred independent of PPAR $\alpha$ status in mouse models. ${ }^{112}$ Differential effects on the immune system have been noted in studies that use a developmental exposure paradigm. Increased sensitivity to DEHP exposure, characterized by altered immune parameters (T-dependent antigen response, natural killer cell activity, and tumor necrosis factor-alpha [TNF- $\alpha$ ] production), was observed in male Wistar rats administered DEHP $(0,1,3,10,30,100,300$, or $1,000 \mathrm{mg} / \mathrm{kg} /$ day $)$ by oral gavage during their juvenile period (postnatal day [PND] 10-50) relative to adult-only exposure (PND 50-90). ${ }^{113}$ In contrast, no persistent effects on numerous assessed immune parameters were noted in a study by Piepenbrink et al. in which CD rats were gestationally exposed to $\operatorname{DEHP}(0,37.5,75,150$, or $300 \mathrm{mg} / \mathrm{kg} /$ day). ${ }^{114}$ In the same study, no DEHP-associated immunotoxicity was noted in nulliparous exposed adults. Topical DEHP administration in adult B6C3F1 female mice did not increase serum concentrations of IgE, interleukin-4 (IL-4), or IL-13, suggesting a limited potential to induce allergic asthma. ${ }^{115}$ Additional studies report dose-dependent increases in 
some inflammatory cell numbers (macrophages, eosinophils, neutrophils, and/or lymphocytes) in bronchoalveolar lavage fluid in $\mathrm{BALB} / \mathrm{c}$ or $\mathrm{BALB} / \mathrm{cJ}$ mice following inhalation exposure to MEHP aerosols. ${ }^{116 ; 117}$ Using median residential indoor air and worst-case exposure concentrations of $0.04 \mu \mathrm{g}$ and $1.2 \mu \mathrm{g} \mathrm{DEHP} / \mathrm{m}^{3}$, respectively, Hansen et al. estimated a margin of exposure between 2,500-75,000, suggesting that immune effects from inhalation exposures are only expected at air concentrations that are well in excess of environmental inhalation exposures typically encountered by humans. ${ }^{116}$

\section{Humans}

Numerous case reports and epidemiological studies suggest a link between phthalate exposure from PVC products and development of allergies and/or asthma. ${ }^{118}$ In a study of 39 PVC-processing plant workers, a higher prevalence of asthma, rhinitis, and eye and respiratory symptoms was observed in individuals exposed to PVC pyrolysis products and phthalates relative to an unexposed reference group. ${ }^{119}$ In a population-based incident case-control study of 521 new asthma cases and 932 control cases, asthma risk was related to the presence of plastic wall materials. ${ }^{120}$ Two epidemiological studies suggest childhood exposure to phthalates via house dust is related to the onset of allergy and/or asthma. In a nested case-control study within a 10,852 child cohort (198 persistent allergic cases, 202 control cases), higher median concentrations of DEHP (cases $-0.828 \mathrm{mg} / \mathrm{g}$ dust; control group $-0.723 \mathrm{mg} / \mathrm{g}$ dust) in house dust were significantly $(\mathrm{p}=0.022)$ associated with asthma. ${ }^{121}$ In a separate study in Bulgarian preschool-age children $(n=102)$, wheezing was associated with higher DEHP concentrations in dust samples collected from children's rooms $(1.24 \mathrm{mg} / \mathrm{g}$ dust for children with wheezing, rhinitis, and/or eczema versus $0.86 \mathrm{mg} / \mathrm{g}$ dust for nonsymptomatic) in the preceding 12-month time interval. ${ }^{122}$ A greater understanding of human exposure relative to animal effect levels and additional mechanistic studies are needed to support a causal inference between DEHP exposure and immunomodulatory effects in humans.

\section{Carcinogenicity}

\section{Experimental Animals}

Multiple rodent studies were identified in the literature that examined the carcinogenic activity of DEHP, all of which initiated exposure once test animals had reached adulthood. Increased incidences of hepatocellular neoplasms have been corroborated across multiple rodent studies, along with reports of increased incidences of testicular Leydig cell tumors and pancreatic acinar adenomas in male rats following chronic exposure to DEHP. In 2-year cancer bioassays conducted by NTP, F344 rats and $\mathrm{B} 6 \mathrm{C} 3 \mathrm{~F} 1$ mice were administered diets containing $0,6,000$, or 12,000 ppm DEHP and 0, 3,000, or 6,000 ppm DEHP, respectively. ${ }^{69 ;}{ }^{123}$ DEHP was found to be carcinogenic in both $\mathrm{F} 344$ rats and $\mathrm{B} 6 \mathrm{C} 3 \mathrm{~F} 1$ mice on the basis of increased incidences of hepatocellular adenomas/carcinomas or neoplastic nodules in both males and females. Significantly increased incidences of hepatocellular adenoma or carcinoma (combined) in F344 rats and B6C3F1 mice were observed at lower DEHP exposure concentrations (male rats: 2,500 ppm; male mice: 500 ppm; female mice: $1,500 \mathrm{ppm}$ ) in chronic studies by David et al. ${ }^{86}$; 124; 125 Additionally, incidences of pancreatic acinar adenomas were increased in male F344 rats at the highest tested exposure concentration $(12,500 \mathrm{ppm}) .{ }^{86}$ A 159 -week study in male Sprague Dawley (SD-CD) rats found that the high-exposure concentration of DEHP $(6,000 \mathrm{ppm}$, or $300 \mathrm{mg} / \mathrm{kg}$ /day) increased the incidence of benign Leydig cell tumors. ${ }^{126}$ 
In rats, the combination of hepatocellular, pancreatic, and testicular tumors is often referred to as the "tumor triad" and is associated with sustained peroxisome proliferator activity. ${ }^{127}$ Although the definitive mode of action of DEHP-mediated carcinogenesis is undetermined, several key events, including activation of PPAR $\alpha$, perturbation of cellular proliferation and apoptosis, selective clonal expansion, and oxidative stress, are hypothesized to contribute to the onset of tumorigenesis.

\section{Humans}

The carcinogenic activity of DEHP in humans has been reviewed by numerous federal and international agencies. In the 14th Report on Carcinogens published by NTP, DEHP was listed as reasonably anticipated to be a human carcinogen based on sufficient evidence of carcinogenicity in experimental animals. ${ }^{128}$ EPA classified DEHP as a Group B2 carcinogen, probable human carcinogen, based on clear evidence of DEHP-mediated induction of liver tumors in rodent models. ${ }^{35}$ The International Agency for Research on Cancer (IARC) previously classified DEHP as "not classifiable as to its carcinogenicity to humans" (Group 3). ${ }^{73}$ The IARC determination was based on two assumptions: (1) that DEHP-induced hepatocellular cancer in rodents occurred as a result of induced peroxisome proliferation activity, and (2) that this mechanism is not relevant to humans due to lower PPAR $\alpha$ expression and lack of observable peroxisome proliferation phenotypes in humans following exposure to known PPAR ligands. However, in light of new information about mechanisms of action, in 2011 IARC reclassified DEHP as a Group 2B carcinogen, indicating that there is "sufficient evidence of carcinogenicity in experimental animals" in combination with "no or limited epidemiological data." 129 The reevaluation included consideration of recent studies with novel transgenic mouse models, such as PPAR $\alpha$-null mice, humanized PPAR $\alpha$ mice, and mice that express a constitutively active PPAR $\alpha$ isoform in hepatocytes. ${ }^{130-132}$ These studies indicate that DEHP can induce hepatocarcinogenesis through a PPAR $\alpha$-independent mechanism, and that other molecular signaling pathways, not just activation of PPAR $\alpha$, likely contribute to the development of cancer.

Epidemiological studies that investigate a link between DEHP exposure and cancer endpoints are limited. In a case-control study of female breast cancer patients, increased cancer risk was associated with elevated urinary concentrations of the DEHP metabolite MECPP, but not other identified DEHP metabolites. ${ }^{133}$ Additional cancer epidemiology studies have been conducted in occupational groups where subjects had worked in PVC processing and plastic manufacturing facilities where increased exposure to phthalate plasticizers was probable. ${ }^{134-140}$ However, many of these studies lacked analytical assessment of exposure to specific phthalates, limiting the ability to determine a causal relationship between DEHP exposure and human cancer.

\section{Genetic Toxicity}

The genetic toxicity of DEHP has been extensively investigated and reviewed over many years (e.g., Huber et al., IARC, and Caldwell). ${ }^{141-143}$ Overall, DEHP shows limited evidence of genotoxic potential, and for the sporadic positive results that have been reported, associations are weak, not reproducible, obtained in a nonstandard test system, or qualified to some degree by the authors. MEHP, one of the main DEHP metabolites, does elicit positive responses, however, in some genotoxicity assays. An early study reported increases in revertant colonies in Salmonella typhimurium strain TA100 and Escherichia coli strain WP2 B/r treated with 2.5 and $5.0 \mathrm{mM}$ MEHP, doses that induced marked cytotoxicity. ${ }^{144}$ More recently, MEHP was reported to 
generate reactive oxygen species and, consequently, DNA strand breaks in cultured AS52 cells ${ }^{145}$ as well as in cultured mouse Leydig tumor cells and in human prostate adenocarcinoma cells as measured by the comet assay. ${ }^{146 ; 147}$ In both cell lines in the latter two studies, the parent compound DEHP ( $3 \mathrm{mM}$ for 24 hours) was also reported to induce DNA damage, although the concentration tested was 1,000 times higher than the concentration tested of MEHP (3 uM). Similarly, DNA damage, measured by the comet assay, was also reported for DEHP in cultured HeLa cells treated with $96.6 \mu \mathrm{M}$ DEHP for 24 hours. $^{148}$

NTP has conducted several in vitro and in vivo genotoxicity assays with DEHP. Unpublished NTP data are included in Appendix D of this report. Published NTP studies, results of which are consistent with most published studies, showed no induction of gene mutations in any of several strains of $S$. typhimurium ${ }^{149 ; 150}$ or in cultured mouse lymphoma L5178Y t $k^{+/-}$cells. $^{151}$ Additional bacterial mutation studies also reported negative results (e.g., Simon et al. ${ }^{152}$ ). Cytogenetic studies in cultured Chinese hamster ovary cells were negative for induction of chromosomal aberrations and were either positive or equivocal for induction of sister chromatid exchanges. ${ }^{153}$ In vitro chromosomal aberration studies, not conducted by NTP, in human leukocytes and human fetal lung cells with DEHP also showed no significant increases in chromosomally aberrant cells, ${ }^{154}$ as did chromosome aberration studies in Chinese hamster cells. ${ }^{155-157}$ Studies that assessed the ability of DEHP to induce sex-linked recessive lethal mutations in germ cells of male Drosophila melanogaster after exposure of either adults (via injection) or larvae (via feeding) were negative. ${ }^{158 ; 159}$

Although sporadic reports of DNA damage or chromosomal effects following in vitro exposure to DEHP exist, results from in vivo studies are almost uniformly negative. In an in vivo comet assay conducted as part of the Japanese led multi-laboratory international validation effort for the assay, DEHP, administered by gavage at a top dose of 2,000 $\mathrm{mg} / \mathrm{kg} /$ day for 3 days, did not induce DNA damage in cells obtained from the stomach, liver, and bone marrow of male Sprague Dawley rats. ${ }^{160}$ In addition, bone marrow samples from those rats showed no increase in the percentage of micronucleated erythrocytes, which are biomarkers of chromosomal damage. In another study, no increases in the frequencies of gpt and Spi(-) mutations were seen in DNA extracted from liver cells of gpt transgenic rats (both F344 and Sprague Dawley backgrounds) with exposure of up to 12,000 ppm DEHP exposure in the diet for 4 weeks, a concentration that produced generalized toxicity (e.g., increased liver weights). ${ }^{161}$ Similarly, an earlier study found that a 13-week Sprague Dawley gpt delta transgenic rats exposed to 12,000 ppm DEHP in the diet resulted in no increases in mutations in liver cell DNA. ${ }^{162}$

A study designed to investigate the potential for DEHP to induce unscheduled DNA synthesis (UDS) in liver cells of male B6C3F1 mice-a species that is sensitive to tumor induction by DEHP — found that exposures up to $500 \mathrm{mg} / \mathrm{kg}$ DEHP acutely or $6,000 \mathrm{ppm}$ in the diet for up to 28 days did not induce UDS, measured using autoradiographic methods. ${ }^{163}$ The investigators also treated primary mouse hepatocytes in culture with up to $1.0 \mathrm{mM}$ DEHP and observed no UDS at time points ranging from $12-48$ hours posttreatment.

The negative results from the in vivo studies described above contrast with an earlier study by Singh et al. that reported a weak positive response in an in vivo rodent dominant lethal test using ICR mice. In that study, DEHP was administered via intraperitoneal injection at $66 \%$ of the acute LD 50 dose, determined as $38.35 \mathrm{~mL} / \mathrm{kg} .{ }^{164}$ However, Jäckh et al. ${ }^{165}$ reported that a second rodent dominant lethal test that used similar doses of DEHP but administered the chemical via oral 
gavage showed no induction of dominant lethal mutations. They therefore suggested that the weak positive response in the Singh et al. study was likely related to nongenotoxic mechanisms, as covalent binding to DNA was not detected in liver cells of rats administered ${ }^{14} \mathrm{C}$ - and ${ }^{3} \mathrm{H}-$ labeled DEHP $(500 \mathrm{mg} / \mathrm{kg})$ by gavage. ${ }^{165}$

\section{Study Rationale}

In response to data gaps related to in utero and early life phthalate exposure and resultant adverse reproductive, developmental, and carcinogenic effects, NTP initiated a cancer hazard assessment for lifetime exposure to environmental phthalates. For DEHP, studies in rodents have established the gestational period as the time of greatest sensitivity to adverse developmental effects, specifically dysmorphogenesis of the male reproductive system. DEHP is a known rodent carcinogen; however, no previous cancer assessments have included exposure during the perinatal period (gestation and lactation). Therefore, it is unknown whether the carcinogenic response is altered when lifetime exposure encompasses these critical developmental windows.

NTP designed two 2-year studies in rats to evaluate whether DEHP lifetime exposure, including during the perinatal developmental period, would alter the dose response of the carcinogenic response relative to postweaning-only exposure. In these studies, DEHP was administered in dosed feed to mimic a common route of human exposure. 


\section{Materials and Methods}

\section{Procurement and Characterization of Di(2-ethylhexyl) Phthalate}

Di(2-ethylhexyl) phthalate (DEHP) was obtained from Aldrich Chemical Company Inc. (St. Louis, MO) in a single lot (lot $01514 \mathrm{TH}$ ) that was used in both 2-year studies. Identity, purity, and stability analyses were conducted by the analytical chemistry laboratory at RTI International (Research Triangle Park, NC) (Appendix A). Reports on analyses performed in support of the DEHP studies are on file at the National Institute of Environmental Health Sciences (NIEHS).

Lot $01514 \mathrm{TH}$ of the chemical, a clear liquid, was identified as DEHP by infrared (IR) spectroscopy, ${ }^{1} \mathrm{H}$ and ${ }^{13} \mathrm{C}$ nuclear magnetic resonance (NMR) spectroscopy, gas chromatography (GC) with mass spectrometry (MS) detection, and high-resolution MS (HRMS) (Table A-1). The IR spectrum was in good agreement with a reference spectrum and the structure was consistent with DEHP. Both ${ }^{1} \mathrm{H}$ and ${ }^{13} \mathrm{C}$ NMR spectra were consistent with reference and predicted spectra. The GC/MS spectra correlated well with the structure of DEHP and the HRMS resulted in measured mass within $0.5 \mathrm{ppm}$ of the theoretical value. The elemental analysis was consistent with the composition of DEHP.

Karl Fisher titration determined the water content of lot $01514 \mathrm{TH}$ to be $0.145 \%$. Ultra-performance liquid chromatography (UPLC) with photodiode array (PDA) detection and GC with flame ionization detection (FID) were used to determine a purity of $99.7 \%$ (Table A-1). The UPLC/PDA and GC/FID showed a minor peak accounting for $0.2 \%$ and $0.3 \%$, respectively, of the total response in the chromatograms. Therefore, bulk purity was determined to be $>99 \%$.

Accelerated stability studies confirmed that lot $01514 \mathrm{TH}$ was stable for at least 2 weeks when stored in sealed glass vials at $5^{\circ} \mathrm{C}$ and $60^{\circ} \mathrm{C}$. The bulk chemical of lot $01514 \mathrm{TH}$ was homogenized by shaking each of the $50 \mathrm{~L}$ plastic jugs for approximately 2 minutes and then transferred to $4 \mathrm{~L}$ amber glass storage bottles, which were stored at room temperature. Periodic reanalysis of the bulk chemical was performed during the studies by the study laboratory using high-performance liquid chromatography (HPLC) with ultraviolet (UV) detection, and no degradation was detected (Table A-1).

\section{Preparation and Analysis of Dose Formulations}

The dose formulations were prepared approximately monthly by mixing DEHP with NIH-07 or NTP-2000 feed (Table A-2). Both the perinatal and postweaning study (Study 1) and the postweaning-only study (Study 2) used dose formulations of 300, 1,000, 3,000, and 10,000 ppm. Formulations were stored in sealed plastic bag-lined containers at room temperature (approximately $25^{\circ} \mathrm{C}$ ) for up to 42 days. The plastic bags used by the study laboratory in the preparation and storage of blank and dosed feed were determined to have no DEHP above the limit of detection of the assay (1.27 ppm).

Homogeneity studies of the dose formulations in a 72-kg NIH-07 feed batch (300 and $10,000 \mathrm{ppm})$ and in a 92-kg NTP-2000 feed batch $(300,3,000$, and 10,000 ppm) were performed prior to animal studies by the study laboratory with HPLC/UV (Table A-1). Formulations were 
determined to be homogenous and stable for 42 days at room temperature and under simulated dosing conditions.

Periodic analysis of the DEHP dose formulations was conducted by the study laboratory using HPLC/UV to determine purity and concentration (Table A-3, Table A-4). All preadministration dose formulations were within $10 \%$ of the target concentrations. For the perinatal and postweaning study (Study 1), all postadministration dose formulations of DEHP were within $10 \%$ of target concentrations. In the postweaning-only study (Study 2), one sample collected from the residual feed in the feeder was below $10 \%$ of the target concentration $(-12.3 \%)$. All other postadministration values were within $10 \%$ of the target concentrations.

\section{Animal Source}

Time-mated $\left(\mathrm{F}_{0}\right)$ female Sprague Dawley $\left(\mathrm{Hsd}\right.$ :Sprague Dawley $\left.{ }^{\circledR} \mathrm{SD}^{\circledR}\right)$ rats were obtained from Envigo (formerly Harlan Laboratories, Inc., Indianapolis, IN) for use in the perinatal and postweaning study (Study 1). Weanling (4 to 5 weeks old) male and female Sprague Dawley (Hsd:Sprague Dawley ${ }^{\circledR} \mathrm{SD}^{\circledR}$ ) rats were also obtained from Envigo for use in the postweaningonly study (Study 2).

\section{Animal Welfare}

Animal care and use are in accordance with the Public Health Service Policy on Humane Care and Use of Animals. All animal studies were conducted in an animal facility accredited by AAALAC International. Studies were approved by the Battelle (Columbus, OH) Animal Care and Use Committee and conducted in accordance with all relevant National Institutes of Health (NIH) and National Toxicology Program (NTP) animal care and use policies and applicable federal, state, and local regulations and guidelines.

\section{Two-year Studies}

\section{Exposure Concentration Selection Rationale}

Dietary exposure concentrations of $0,300,1,000,3,000$, or 10,000 ppm DEHP were selected based on previous data from an NTP multigenerational reproductive assessment by continuous breeding (RACB) study, which included a perinatal exposure paradigm in the Sprague Dawley rat model. In the RACB study, the highest tested exposure concentration (10,000 ppm) was welltolerated by pregnant dams and did not affect litter size or pup survival to weaning. However, this exposure concentration induced significant numbers of reproductive tract and testicular malformations in the $\mathrm{F}_{1}$ male offspring and perturbed developmental androgen signaling as evidenced by decreased anogenital distance (AGD) and delayed attainment of puberty. In a previous NTP cancer bioassay using Fischer 344 (F344) rats, increased incidences of hepatocellular neoplasms occurred at exposure concentrations of 6,000 and 12,000 ppm DEHP. Together, these data suggest that the selected exposure concentrations are likely to induce a carcinogenic response and adequately challenge developmentally exposed test animals. To facilitate comparison of the results of the two 2-year studies, with and without perinatal exposure, both studies used the same exposure concentrations. 


\section{Perinatal and Postweaning Study in Rats (Study 1)}

$\mathrm{F}_{0}$ female rats were 12 to 14 weeks old upon receipt. Evidence of mating is defined as gestation day (GD) 1; $F_{0}$ females were received on GD 2 and held for 4 days. $F_{0}$ females were randomly assigned to one of five exposure groups on GD $5(n=45 /$ group). Randomization was stratified by body weight that produced similar group mean weights using PATH/TOX SYSTEM software (Xybion Medical Systems Co., Cedar Knolls, NJ).

$\mathrm{F}_{0}$ females were quarantined for 11 days after receipt. Ten nonmated females received with the time-mated females were designated for disease monitoring 11 days after arrival; samples were collected for serological analyses and the rats were euthanized, necropsied, and examined for the presence of disease or parasites. The health of the $\mathrm{F}_{1}$ rats was monitored during the study according to the protocols of the NTP Sentinel Animal Program (Appendix C). Pinworms (Syphacia spp.) were diagnosed in sentinel animals during routine health monitoring evaluations. Infected animals did not display clinical signs and no pathological lesions were noted in relation to the presence of the pinworms. Study animals did not receive medication for potential pinworm infection. Following this finding, NTP, in coordination with the testing laboratory, developed and implemented a successful plan of pinworm containment and eradication. NTP required the testing laboratories to actively monitor animals to ensure the continued exclusion of pinworms from all studies going forward. All other test results were negative.

Beginning on GD 6, Fo time-mated female rats were fed diets containing 0, 300, 1,000, 3,000, or $10,000 \mathrm{ppm}$ DEHP throughout gestation and lactation. Groups of $50 \mathrm{~F}_{1}$ rats/sex/exposure concentration continued on in the study after weaning and were fed diets containing the same respective DEHP concentration for 2 years.

$\mathrm{F}_{0}$ female rats were housed individually during gestation and with their respective litters during lactation. Water and dosed feed were available ad libitum. $\mathrm{F}_{0}$ females were weighed on GDs 5, 6, $9,12,15,18$, and 21 and on lactation days (LDs) 1, 4, 7, 14, and 21. During gestation, feed consumption was continuously measured over 3-day intervals from GD 6 through GD 21 (GD 69, 9-12, 12-15, 15-18, and 18-21). The day of parturition was considered to be LD 0 . On apparent GD 26, all time-mated female rats that failed to deliver were euthanized and the uteri were examined and stained for evidence of implantation. Total litter weight and litter weights by sex were collected on postnatal day (PND) 1. Individual F1 pups were weighed on PNDs 4, 7, 14, and 21. Clinical observations and survival were evaluated throughout lactation. During lactation, feed consumption was continuously measured over 3-day intervals from LD 1 through LD 21 (LD 1-4, 4-7, 7-10, 10-14, 14-17, 17-21).

Select dams and their litters were removed on GD 18 to quantify mono(2-ethylhexyl) phthalate plasma and tissue concentrations. On GD 18, blood was collected from the retroorbital sinus of randomly selected dams ( $\mathrm{n}=5$ per exposure group). Blood samples were collected in tubes containing $\mathrm{K}_{3}$ EDTA (tripotassium ethylene diamine tetraacetic acid), centrifuged, and the plasma harvested. Amniotic fluid was collected and pooled by dam, and each dam's fetuses were collected and pooled by litter. All samples were flash frozen in liquid nitrogen and stored frozen at approximately $-20^{\circ} \mathrm{C}$ before shipment to RTI International (Research Triangle Park, NC). All samples were analyzed using a validated analytical method (Appendix E).

On PND 4, all litters with surviving pups were retained. Before weaning, two males and two females per litter from 25 litters in the $0,300,1,000$, and 3,000 ppm groups and from 21 litters in 
the $10,000 \mathrm{ppm}$ group were randomly assigned to continue on in the 2-year postweaning phase of the study. To complete assignment in the 10,000 ppm group, two male pups and three female pups were selected from two litters, and two male pups and one female pup were selected from two additional litters. After assignments to the 2-year study were complete, 20 pups per sex from the remaining control pups were randomly selected as the sentinel animals. On the day the last litter reached PND 18, litters were randomly selected and $F_{1}$ pups from these litters were randomly selected for the 2-year study. On the day the last litter reached PND 21, dams were removed and the pups were weaned. Weaning marked the beginning of the 2-year chronic phase of the study.

After weaning, $F_{1}$ pups were housed up to two (males) or four (females) per cage. Dosed feed and water were available ad libitum. Feed consumption was measured weekly for the first 13 weeks and at 4-week intervals thereafter. Cages were changed weekly through PND 4, then changed at least twice weekly. Racks were changed and rotated at least every 2 weeks. Further details of animal maintenance are given in Table 1.

Two diets were utilized in this study: (1) NIH-07 during the perinatal phase, and (2) NTP-2000 during the postweaning phase. The NIH-07 diet is a higher protein diet that supports reproduction and lactation in rodents, whereas the NTP-2000 diet is a lower protein diet that decreases the incidence of chronic nephropathy in adult rats. Information on feed composition and contaminants for both diets is provided in Appendix B.

\section{Postweaning-only Study in Rats (Study 2)}

Male and female rats were 4 to 5 weeks old upon receipt and quarantined for 13 days prior to study start. Rats were randomly assigned to one of five exposure groups ( $\mathrm{n}=50 \mathrm{rats} / \mathrm{sex} / \mathrm{exposure}$ group). Randomization was stratified by body weight that produced similar group mean weights using PATH/TOX SYSTEM software (Xybion Medical Systems Corporation, Cedar Knolls, NJ). Rats were 6 to 7 weeks old on the first day of the study and were provided DEHP in dosed feed for 2 years at one of five exposure concentrations $(0,300,1,000$, $3,000$, or $10,000 \mathrm{ppm})$.

Five male and five female rats were designated for disease monitoring 13 days after arrival; samples were collected for serological analyses, and the rats were euthanized, necropsied, and examined for the presence of disease or parasites. The health of the rats was monitored during the study according to the protocols of the NTP Sentinel Animal Program (Appendix C). Pinworms (Syphacia spp.) were diagnosed in sentinel animals during routine health monitoring evaluations. All other test results were negative.

Rats were housed up to two (males) or four (females) per cage. Water and dosed feed were available ad libitum. Feed consumption was measured weekly for the first 13 weeks and at 4-week intervals thereafter. Cages were changed at least twice weekly. Racks were changed and rotated at least every 2 weeks. Further details of animal maintenance are given in Table 1. Information on feed composition and contaminants is given in Appendix B.

\section{Clinical Examinations and Pathology}

In both of the 2-year studies, animals were observed twice daily for morbidity and moribundity. Animals were weighed initially, weekly for the next 13 weeks, every 4 weeks thereafter, and at 
study termination. Beginning on study day 36 (Study 1) or study week 5 (Study 2), clinical observations were recorded every 4 weeks and at the end of the studies.

Complete necropsies and microscopic examinations were performed on all $F_{1}$ rats in Study 1 and all rats in Study 2. At necropsy, all organs and tissues were examined for grossly visible lesions, and all major tissues were fixed and preserved in 10\% neutral buffered formalin except for eyes, testes, vaginal tunics, and epididymides, which were first fixed in Davidson's solution or modified Davidson's solution. Tissues were processed and trimmed, embedded in paraffin, sectioned at a thickness of 4 to $6 \mu \mathrm{m}$, and stained with hematoxylin and eosin (H\&E) for microscopic examination. For all paired organs (e.g., adrenal gland, kidney, ovary), samples from each organ were examined. In the original evaluation of the uterus, a transverse section through each uterine horn, approximately $0.5 \mathrm{~cm}$ cranial to cervix, was collected for histopathology evaluation. For the residual tissue evaluation of the uterus, all remaining uterine, including the cervix, and vaginal tissue was sectioned longitudinally and examined histologically. Results from the residual uterine evaluation were combined with those from the original, transverse section of uterus. Tissues examined microscopically are listed in Table 1.

Microscopic evaluations were completed by the study laboratory pathologist, and the pathology data were entered into the Toxicology Data Management System. The report, slides, paraffin blocks, residual wet tissues, and pathology data were sent to the NTP Archives for inventory, slide/block match, wet tissue audit, and storage. The slides, individual animal data records, and pathology tables were evaluated by quality assessment (QA) pathologists at a pathology laboratory independent of the study laboratory. The individual animal records and tables were compared for accuracy, the slide and tissue counts were verified, and the histotechnique was evaluated. For both 2-year studies, the QA pathologists evaluated slides from all neoplasms and all potential target organs, which included the liver, pancreas, kidney, heart, bone marrow, and pituitary gland of rats; testes and epididymis of male rats; and the uterus of female rats. Kidney pathology is reported only for Study 1. Additional sex-specific target tissues identified in Study 1 included the prostate glands, gubernacula, phallus, prepuce, seminal vesicles, and vagina.

The QA report and the reviewed slides were submitted to the NTP Pathology Working Group (PWG) coordinator, who reviewed the selected tissues and addressed any inconsistencies in the diagnoses made by the laboratory and QA pathologists. Representative histopathology slides containing examples of lesions related to chemical administration, examples of disagreements in diagnoses between the laboratory and QA pathologists, or lesions of general interest were presented by the QA/PWG coordinators to the PWG for review. The PWG consisted of the QA pathologists and other pathologists experienced in rodent toxicological pathology. The PWG examined the tissues without any knowledge of exposure groups. When the PWG consensus diagnosis differed from that of the laboratory pathologist, the diagnosis was changed. Final diagnoses for reviewed lesions represent a consensus between the laboratory pathologist, reviewing pathologist(s), and the PWG. Details of these review procedures have been described, in part, by Maronpot and Boorman ${ }^{166}$ and Boorman et al. ${ }^{167}$ For subsequent analyses of the pathology data, the decision whether or not to evaluate the diagnosed lesions for each tissue type separately or combined was generally based on the guidelines of McConnell et al. ${ }^{168}$ 
Table 1. Experimental Design and Materials and Methods in the Two-year Feed Studies of Di(2-ethylhexyl) Phthalate

Perinatal and Postweaning Study (Study 1)

Postweaning-only Study (Study 2)

Study Laboratory

Battelle (Columbus, $\mathrm{OH}$ )

Same as Study 1

Strain and Species

Sprague Dawley (Hsd:Sprague Dawley ${ }^{\circledR} \mathrm{SD}^{\circledR}$ ) rats

Same as Study 1

Animal Source

Envigo (formerly Harlan Laboratories, Inc., Indianapolis, IN)

Same as Study 1

\section{Time Held Before Studies}

$\mathrm{F}_{0}$ female rats: 4 days

14 or 15 days

\section{Average Age When Studies Began}

$F_{0}$ female rats: 13 to 15 weeks

6 to 7 weeks

\section{Date of First Exposure}

$\mathrm{F}_{0}$ female rats: May 20, 2011

February 17 (males) or 18 (females), 2011

$F_{1}$ rats: June 27 (males) or 28 (females), 2011

Duration of Exposure

$\mathrm{F}_{0}$ female rats: GD 6 to LD 21

2 years

$\mathrm{F}_{1}$ : Perinatal plus 2 years

\section{Date of Last Exposure}

$\mathrm{F}_{0}$ female rats: June 27, 2011

February 21 (males) or 27 (females), 2013

$F_{1}$ rats: June 27 (males) or July 3 (females), 2013

\section{Necropsy Dates}

$F_{1}$ rats: June 24 to 27 (males) or June 28 to July 3 (females), 2013

February 18 to 21 (males) or February 22 to 27 (females), 2013

Average Age at Necropsy

$F_{1}$ rats: 2 years

2 years

Size of Study Groups

$\mathrm{F}_{0}$ female rats: 45

$50 / \operatorname{sex}$

$\mathrm{F}_{1}$ rats: $50 /$ sex

\section{Method of Distribution}

Animals were distributed randomly into groups of approximately equal initial mean body weights

Same as Study 1

\section{Animals per Cage}

$\mathrm{F}_{0}$ female rats: 1 (with litter)

2 (males) or 4 (females) 


\section{Method of Animal Identification}

$\mathrm{F}_{0}$ female rats: Cage card and tail marking with permanent pen

$\mathrm{F}_{1}$ (pups): Limb tattoo

$\mathrm{F}_{1}$ rats (2-year study): Cage card and tail tattoo

\section{Diet}

Irradiated NIH-07 meal feed (perinatal phase) or irradiated NTP-2000 meal feed (2-year study) (Zeigler Brothers, Inc, Gardners, PA), available ad libitum, changed twice weekly

\section{Water}

Tap water (Columbus municipal supply) via automatic watering system (Edstrom Industries, Inc., Waterford, WI), available ad libitum

\section{Cages}

Solid polycarbonate (Lab Products, Inc., Seaford, DE); changed weekly through PND 4, then at least twice weekly; rotated every 2 weeks

\section{Bedding}

Irradiated Sani-Chips ${ }^{\circledR}$ (P.J. Murphy Forest Products

Corporation, Montville, NJ), changed with cage changes

\section{Rack Filters}

Spun-bonded polyester (Snow Filtration Company, Cincinnati, OH, or National Media Filter Corporation, Olive Branch, MS), changed every 2 weeks

\section{Racks}

Stainless steel (Lab Products, Inc., Seaford, DE), changed and rotated at least every 2 weeks

\section{Animal Room Environment}

Temperature: $72^{\circ} \mathrm{F} \pm 3^{\circ} \mathrm{F}$

Relative humidity: $50 \% \pm 15 \%$

Room fluorescent light: 12 hours/day

Room air changes: at least 10/hour

\section{Exposure Concentrations}

$0,300,1,000,3,000$, and $10,000 \mathrm{ppm}$ in feed
Cage card and tail tattoo

Irradiated NTP-2000 meal feed (Zeigler Brothers, Inc., Gardners, PA), available ad libitum, changed twice weekly

Same as Study 1

Solid polycarbonate (Lab Products, Inc., Seaford, DE); changed twice weekly; rotated every 2 weeks

Same as Study 1

Same as Study 1

Same as Study 1

Same as Study 1

Same as Study 1 
Perinatal and Postweaning Study (Study 1)

\section{Type and Frequency of Observation}

$F_{0}$ female rats: Observed twice daily. Weighed on GDs 5, 6, 9, 12, 15, 18, and 21 and on LDs 1, 4, 7, 14, and 21 . Feed consumption was measured over 3-day intervals from GD 6 to GD 21 and LD 1 to LD 21.

$F_{1}$ rats: Observed twice daily. Litter data (total litter weight, litter weights by sex, and litter observations) were recorded on PND 1. Pup survival was evaluated and recorded. Individual pups were weighed on PNDs 4, 7,14 , and 21 , weekly for the first 13 weeks after weaning, every 4 weeks thereafter, and at the end of the study. Clinical findings were recorded every 4 weeks beginning on day 36 and at the end of the study. Feed consumption was recorded weekly for the first 13 weeks and at 4-week intervals thereafter.

\section{Method of Euthanasia}

Carbon dioxide

\section{Necropsy}

Necropsies were performed on all $F_{1}$ animals.

\section{Histopathology}

Complete histopathology was performed on all rats. In addition to gross lesions and tissue masses, the following tissues were examined: adrenal gland, bone with marrow, brain, clitoral gland, esophagus, eyes, Harderian gland, heart, large intestine (cecum, colon, rectum), small intestine (duodenum, jejunum, ileum), kidneys, liver, lung, lymph nodes (mandibular and mesenteric), mammary gland, nose, ovary, pancreas, parathyroid gland, pituitary gland, preputial gland, prostate gland, salivary gland, seminal vesicle, spleen, stomach (forestomach and glandular), testis with epididymis, thymus, thyroid gland, trachea, urinary bladder, uterus, and vagina.

\section{Internal Dose Assessment}

Maternal plasma, amniotic fluid, and fetal (pooled by
Postweaning-only Study (Study 2)

Observed twice daily. Body weights were recorded initially, weekly for the first 13 weeks, every 4 weeks thereafter, and at the end of the study. Clinical findings were recorded every 4 weeks beginning at week 5 and at the end of the study. Feed consumption was recorded weekly for the first 13 weeks and at 4-week intervals thereafter.
Same as Study 1

Necropsies were performed on all animals.

Same as Study 1

litter) mono(2-ethylhexyl) phthalate concentrations were measured at GD 18.

$\overline{\mathrm{GD}}=$ gestation day; $\mathrm{LD}$ = lactation day; $\mathrm{PND}=$ postnatal day.

\section{Benchmark Dose Analysis}

Benchmark doses (BMDs) were calculated using the EPA Benchmark Dose Software (BMDS), version 3.1.2. ${ }^{169}$ and are presented in Appendix F. The dose variable for the models was the amount of DEHP consumed in $\mathrm{mg} / \mathrm{kg}$ body weight/day ( $\mathrm{mg} / \mathrm{kg} /$ day). Numbers of animals per exposure group were poly-3-adjusted survival numbers. The response variable was the incidence of the endpoint being modeled. 
All of the frequentist dichotomous models in the BMDS were used. The logistic, log-probit, and probit models were used with no parameter restrictions. Other models (dichotomous Hill, gamma, log-logistic, multistage, and Weibull) were used with default restrictions on the ranges of some of the parameters, as described in the BMDS User Guide. ${ }^{170}$

The benchmark response (BMR) used in the models was $0.1(10 \%)$ extra risk, with estimated background levels. The benchmark dose lower confidence limit (BMDL) was calculated using a $95 \%$ confidence interval. The decision logic used to recommend one model from the fitted models was the default logic. ${ }^{170}$

\section{Statistical Methods}

\section{Survival Analyses}

The probability of survival was estimated by the product-limit procedure of Kaplan and Meier ${ }^{171}$ and is presented graphically. Animals surviving to the end of the observation period are treated as censored observations, as are animals dying from unnatural causes within the observation period. Animals dying from natural causes are included in analyses and are treated as uncensored observations. For the postweaning-only study (Study 2), exposure concentration-related trends are identified with Tarone's life-table test, ${ }^{172}$ and pairwise exposure concentration-related effects are assessed using Cox's method. ${ }^{173}$ For the perinatal and postweaning study (Study 1), exposure concentration-related trends and pairwise exposure-related effects on survival are assessed using a Cox proportional hazards model ${ }^{173}$ with a random litter effect. All reported $p$ values for the survival analyses are two-sided.

\section{Calculation of Incidence}

The incidences of neoplasms or nonneoplastic lesions are presented as the numbers of animals bearing such lesions at a specific anatomic site. For calculation of incidence rates, the denominator for most neoplasms and all nonneoplastic lesions is the number of animals where the site was examined microscopically. When macroscopic examination was required to detect neoplasms in certain tissues (e.g., mesentery, pleura, peripheral nerve, skeletal muscle, tongue, tooth, and Zymbal's gland) before microscopic evaluation, however, the denominator consists of the number of animals that had a gross abnormality. When neoplasms had multiple potential sites of occurrence (e.g., leukemia or lymphoma), the denominator consists of the number of animals on which a necropsy was performed. Additional study data also give the survival-adjusted neoplasm rate for each group and each site-specific neoplasm. This survival-adjusted rate (based on the Poly-3 method described below) accounts for differential mortality by assigning a reduced risk of neoplasm, proportional to the third power of the fraction of time on study, only to site-specific, lesion-free animals that do not reach terminal euthanasia.

\section{Analysis of Neoplasm and Nonneoplastic Lesion Incidence}

Statistical analyses of neoplasm and nonneoplastic lesion incidence considered two features of the data. Some animals did not survive the entire 2 years of the study, so survival differences between groups had to be considered. Also, up to two animals per sex were randomly selected from each litter to participate in the study, except for the 10,000 ppm group in the perinatal and postweaning study (Study 1) for which additional males and females were needed to populate the 
study. The statistical analysis of lesion incidence used the Poly-3 test to account for survival differences, with a Rao-Scott adjustment for litter effects, as described below.

The Poly-k test ${ }^{174-176}$ was used to assess neoplasm and nonneoplastic lesion prevalence. This test is a survival-adjusted quantal-response procedure that modifies the Cochran-Armitage linear trend test to account for survival differences. More specifically, this method modifies the denominator in the quantal estimate of lesion incidence to approximate more closely the total number of animal years at risk. For analysis of a given site, each animal is assigned a risk weight. This value is 1 if the animal had a lesion at that site or if it survived until terminal euthanasia; if the animal died before terminal euthanasia and did not have a lesion at that site, its risk weight is the fraction of the entire study time that it survived, raised to the kth power.

This method yields a lesion prevalence rate that depends only on the choice of a shape parameter for a Weibull hazard function describing cumulative lesion incidence over time. ${ }^{174}$ Unless otherwise specified, a value of $\mathrm{k}=3$ was used in the analysis of site-specific lesions. This value was recommended by Bailer and Portier ${ }^{174}$ after an evaluation of neoplasm onset time distributions for a variety of site-specific neoplasms in control F344 rats and B6C3F1 mice. ${ }^{177}$ Bailer and Portier ${ }^{174}$ showed that the Poly-3 test provided valid results if the true value of $\mathrm{k}$ is anywhere in the range from 1 to 5. A further advantage of the Poly-3 method is that it does not require lesion lethality assumptions. Variation introduced by the use of risk weights, which reflect differential mortality, was accommodated by adjusting the variance of the Poly-3 statistic as recommended by Bieler and Williams. ${ }^{178}$ Poly-3 tests used the continuity correction described by Nam. ${ }^{179}$

Littermates tend to be more like each other than like fetuses/pups in other litters. Failure to account for correlation within litters leads to underestimates of variance in statistical tests, resulting in higher probabilities of Type I errors ("false positives"). Because up to two pups per sex per litter were present in the perinatal and postweaning study (Study 1), the Poly-3 test was modified to accommodate litter effects using the Rao-Scott approach. ${ }^{180}$ The Rao-Scott approach accounts for litter effects by estimating the ratio of the variance in the presence of litter effects to the variance in the absence of litter effects. This ratio is then used to adjust the sample size downward to yield the estimated variance in the presence of litter effects. The Rao-Scott approach was implemented in the Poly-3 test as recommended by Fung et al., ${ }^{181}$ formula $\overline{\boldsymbol{T}}_{R S 2}$.

Tests of significance included pairwise comparisons of each exposed group with control groups and a test for an overall exposure concentration-related trend. Continuity-corrected Rao-Scottadjusted Poly-3 tests were used in the analysis of lesion incidence and reported $p$ values are onesided. The significance of a lower incidence or negative trend in lesions is approximated as $1-p$ with the letter $\mathrm{N}$ added (e.g., $\mathrm{p}=0.99$ is presented as $\mathrm{p}=0.01 \mathrm{~N}$ ). For neoplasms and nonneoplastic lesions observed without litter structure (e.g., at the interim evaluation), Poly3 tests that included the continuity correction, but without adjustment for potential litter effects, were used for trend and pairwise comparisons to the control group.

To evaluate incidence rates by litter, the proportions of litters affected by each lesion type were tested among groups. The Cochran-Armitage trend test and Fisher's exact test ${ }^{182}$ were used to test for trends and pairwise differences from the control group, respectively. 


\section{Analysis of Continuous Variables}

Before statistical analysis, extreme values identified by the outlier test of Dixon and Massey, ${ }^{183}$ for small samples $(\mathrm{n}<20)$, and Tukey's outer fences method, ${ }^{184}$ for large samples $(\mathrm{n} \geq 20)$, were examined by NTP personnel, and implausible values were eliminated from the analysis. Organ and body weight measurements, which historically have approximately normal distributions, were analyzed with the parametric multiple comparison procedures of Dunnett ${ }^{185}$ and Williams. ${ }^{186 ;} 187$ Dam gestational and lactational feed consumption, litter sizes, pup survival, implantations, number of resorptions, and proportions of male pups per litter for all studies were analyzed using the nonparametric multiple comparison methods of Shirley ${ }^{188}$ [as modified by Williams ${ }^{189}$ ] and Dunn ${ }^{190}$ given that these endpoints typically have skewed distributions. For all quantitative endpoints unaffected by litter structure, the Jonckheere test ${ }^{191}$ was used to assess the significance of exposure concentration-related trends and to determine, at the 0.01 level of significance, whether a trend-sensitive test (the Williams or Shirley test) was more appropriate for pairwise comparisons than a test that does not assume a monotonic exposure concentrationrelated trend (the Dunnett or Dunn test).

Postweaning body weights were measured on two pups/sex/litter in most cases in the perinatal and postweaning study (Study 1); more than two pups/sex/litter were common in preweaning body weight measurements. The analyses of pup mean body weights and mean body weights adjusted for litter size (described below) of these animals took litter effects into account using a mixed model with litter as a random effect. To adjust for multiple comparisons, a Dunnett-Hsu adjustment was used. ${ }^{192}$ Dam mean body weights during gestation and lactation were analyzed with the parametric multiple comparison procedures of Dunnett ${ }^{185}$ and Williams, ${ }^{186 ;} 187$ depending on whether the Jonckheere test indicated the use of a trend-sensitive test. P values for these analyses are two-sided.

\section{Analysis of Gestational and Fertility Indices}

Cochran-Armitage trend tests were used to test the significance of trends in gestational and fertility indices across exposure groups. Fisher's exact test was used to conduct pairwise comparisons of each exposed group with the control group. $\mathrm{P}$ values for these analyses are two-sided.

\section{Body Weight Adjustments}

To adjust preweaning pup body weights for live litter size, a linear model was fit to body weights as a function of exposure concentration and litter size. The estimated coefficient of litter size was then used to adjust each pup body weight on the basis of the difference between its litter size and the mean litter size. Preweaning pup body weights were adjusted for PND 1 live litter size. After adjustment, mean body weights were analyzed with a linear mixed model with a random litter effect.

\section{Historical Control Data}

The concurrent control group is the most valid comparison to the exposed groups and is the only control group analyzed statistically in NTP bioassays. However, historical control data are often helpful in interpreting potential exposure-related effects, particularly for uncommon or rare neoplasm types. For meaningful comparisons, the conditions for studies in the historical control 
data must be generally similar. Significant factors that can affect the background incidence of neoplasms at a variety of sites are diet, sex, strain/stock, and route of exposure. The NTP historical control database contains all 2-year studies for each species, sex, and strain/stock with histopathology findings in control animals completed within the most recent 5-year period, ${ }^{193-195}$ including the concurrent control for comparison across multiple technical reports. In general, the historical control data for a given study includes studies using the same route of administration, and the overall incidence of neoplasms in control animals for all routes of administration are included for comparison, including the current study.

\section{Quality Assurance Methods}

Both the perinatal and postweaning study (Study 1) and the postweaning-only study (Study 2) were conducted in compliance with the Food and Drug Administration Good Laboratory Practice Regulations. ${ }^{196}$ In addition, both study reports were audited retrospectively by an independent QA contractor against study records submitted to the NTP Archives. Separate audits covered completeness and accuracy of the pathology data, pathology specimens, final pathology tables, and a draft of this NTP Technical Report. Audit procedures and findings are presented in the reports and are on file at NIEHS. The audit findings were reviewed and assessed by NTP staff, and all comments were resolved or otherwise addressed during the preparation of this Technical Report.

\section{Genetic Toxicology}

A large number of genetic toxicity tests for DEHP were conducted by NTP. The genetic toxicity data presented here are the culmination of several NTP evaluations of DEHP that have not been previously published. The protocols used for the conduct and evaluation of the in vivo chromosomal aberrations and micronucleus tests are described in detail in Appendix D.

The genetic toxicity studies have evolved from an earlier effort by NTP to develop a comprehensive database permitting a critical anticipation of a chemical's carcinogenicity in experimental animals based on numerous considerations, including the molecular structure of the chemical and its observed effects in short-term in vitro and in vivo genetic toxicity tests (structure-activity relationships). The short-term tests were originally developed to clarify proposed mechanisms of chemical-induced DNA damage on the basis of the relationship between electrophilicity and mutagenicity ${ }^{197}$ and the somatic mutation theory of cancer. ${ }^{198 ; 199}$ It should be noted, however, that not all cancers arise through genotoxic mechanisms.

DNA reactivity combined with Salmonella mutagenicity is highly correlated with induction of carcinogenicity in multiple species/sexes of rodents and at multiple tissue sites. ${ }^{200}$ Information from other in vitro genotoxicity assays does not appear to increase the predictivity of the bacterial mutation assay for rodent carcinogenicity, but these other tests can provide useful information on the types of DNA and chromosomal damage induced by the chemical under investigation. Positive results seen in in vivo assays that measure induction of chromosomal damage have been shown to have a high correlation with rodent carcinogenicity. ${ }^{201}$ 


\section{Results}

\section{Data Availability}

The National Toxicology Program (NTP) evaluated all study data. Data relevant for evaluating toxicological findings are presented here. All study data are available in the NTP Chemical Effects in Biological Systems (CEBS) database: https://doi.org/10.22427/NTP-DATA-TR$601 . .^{202}$

\section{Perinatal and Postweaning Study in Rats (Study 1)}

\section{Perinatal Phase}

No effects on maternal survival were observed following exposure to di(2-ethylhexyl) phthalate (DEHP), and no exposure-related maternal clinical observations were noted (Appendix H). Administration of DEHP had no effects on the percentage of pregnant females that produced pups, gestation length, or pup sex distribution (Table 2; Appendix H). The lower number of females that produced pups in the $10,000 \mathrm{ppm}$ group was due to 13 mated females that were not pregnant. This was not attributed to the test article, because dam exposure to DEHP started after the period of implantation.

Table 2. Summary of the Disposition of $F_{0}$ Female Rats during Perinatal Exposure in the Perinatal and Postweaning Two-year Feed Study of Di(2-ethylhexyl) Phthalate

\begin{tabular}{|c|c|c|c|c|c|}
\hline & 0 ppm & 300 ppm & 1,000 ppm & 3,000 ppm & 10,000 ppm \\
\hline \multicolumn{6}{|l|}{ Reproductive Performance } \\
\hline Time-mated Females (GD 6) & 45 & 45 & 45 & 45 & 45 \\
\hline Females Pregnant $(\%)^{\mathrm{a}}$ & $39(86.7)$ & $35(77.8)$ & $38(84.4)$ & $35(77.8)$ & $32(71.1)$ \\
\hline Females Not Pregnant (\%) & $6(13.3)$ & $10(22.2)$ & $7(15.6)$ & $10(22.2)$ & $13(28.9)$ \\
\hline $\begin{array}{l}\text { Dams Not Delivering with Evidence } \\
\text { of Pregnancy }(\%)\end{array}$ & $5(12.8)$ & $6(17.1)$ & $7(18.4)$ & $6(17.1)$ & $6(18.8)$ \\
\hline Dams with Litters on LD $0(\%)^{\mathrm{a}}$ & $34(87.2)$ & $29(82.9)$ & $31(81.6)$ & $29(82.9)$ & $26(81.2)$ \\
\hline Gestation Length (Days) $^{\mathrm{b}, \mathrm{c}}$ & $22.1 \pm 0.1$ & $22.1 \pm 0.1$ & $22.3 \pm 0.1$ & $22.0 \pm 0.0$ & $22.2 \pm 0.1$ \\
\hline Number of Litters on LD $4^{\mathrm{d}}$ & 34 & 29 & 28 & 29 & 26 \\
\hline Weaned Males/Females & $204 / 213$ & $166 / 178$ & $180 / 166$ & $166 / 173$ & $138 / 117$ \\
\hline \multicolumn{6}{|c|}{$\begin{array}{l}\text { GD }=\text { gestation day; } \mathrm{LD}=\text { lactation day. } \\
\text { a'Statistical analysis performed by the Cochran-Armitage (trend) and Fisher's exact (pairwise) tests. } \\
\text { 'Statistical analysis performed by the Jonckheere (trend) and Shirley or Dunn (pairwise) tests. } \\
\text { 'Gestation length calculated for sperm-positive females that delivered a litter. Data are presented as mean } \pm \text { standard error. } \\
\text { d'Litters were not standardized in this study. }\end{array}$} \\
\hline \multicolumn{6}{|c|}{$\begin{array}{l}\text { Mean gestation body weights of dams receiving up to } 3,000 \text { ppm DEHP in the diet were within } \\
\text { approximately } 3.5 \% \text { of control animals (Table } 3 \text { ). Dams that received } 10,000 \text { ppm DEHP in the } \\
\text { diet displayed significantly decreased mean body weights (up to } 10 \% \text { ), relative to control } \\
\text { animals, throughout the gestational period. Lower relative body weights in } 10,000 \text { ppm dams } \\
\text { were associated with significantly decreased body weight gain over the GD } 6-9 \text {, GD 15-18, and } \\
\text { GD 18-21 intervals (Table 3). During the gestational period (GD 6-21), the overall mean body }\end{array}$} \\
\hline
\end{tabular}


weight gain of $10,000 \mathrm{ppm}$ dams was significantly decreased $27 \%$ compared to that of the control animals. During the lactational period, there were no effects on maternal mean body weight or body weight gain among dam groups receiving up to 3,000 ppm DEHP (Table 3). Lactational body weights of $10,000 \mathrm{ppm}$ dams were significantly decreased (up to approximately $25 \%$ ) at all assessed lactational time points relative to control animals. This decrease was more severe in magnitude than what was observed during gestation and is likely the result of decreases in absolute body weight during the lactational period. All other exposure groups, including the control group, displayed positive weight gains during the lactation day (LD) 4-21 interval, whereas the $10,000 \mathrm{ppm}$ dams lost an average of $24 \mathrm{~g}$ in body weight, corresponding to an approximate $10 \%$ decrease in body weight from LD 1 to LD 21 .

For dams exposed at 10,000 ppm DEHP, significantly decreased feed consumption during both gestation (GD 6-21; approximately 14\%) and lactation (LD 1-14; 39\%), relative to control animals (Table 4), likely contributed to the observed decrements in body weight (Table 3). Decreased feed consumption (8\%) was also observed in 3,000 ppm dams during the LD 17-21 interval, and attained statistical significance, relative to control animals. Gestational DEHP intake (GD 6-21) for dams in the 300, 1,000, 3,000, and 10,000 ppm groups was approximately $21,68,206$, and $626 \mathrm{mg} \mathrm{DEHP} / \mathrm{kg}$ body weight/day ( $\mathrm{mg} / \mathrm{kg} /$ day), respectively (Table 4$)$. Lactational DEHP intake (LD 1-14) for dams in the 300, 1,000,3,000, and 10,000 ppm groups was approximately $49,166,482$, and $1,244 \mathrm{mg} / \mathrm{kg} /$ day, respectively (Table 4 ). Chemical intake for the LD 14-21 interval was not calculated due to the unknown contribution of offspring feed consumption to the overall cage-based measurements. 
Table 3. Summary of Mean Body Weights and Body Weight Gains of $F_{0}$ Female Rats during Gestation and Lactation in the Perinatal and Postweaning Two-year Feed Study of Di(2-ethylhexyl) Phthalate

\begin{tabular}{cccccc}
\hline Parameter $\mathbf{a}, \mathbf{b}$ & $\mathbf{0 ~ p p m}$ & $\mathbf{3 0 0} \mathbf{~ p p m}$ & $\mathbf{1 , 0 0 0} \mathbf{~ p p m}$ & $\mathbf{3 , 0 0 0} \mathbf{~ p p m}$ & $\mathbf{1 0 , 0 0 0} \mathbf{~ p p m}$ \\
\hline Gestation Day & & & & & \\
6 & $237.4 \pm 2.14(39)$ & $236.1 \pm 2.63(35)$ & $234.6 \pm 2.63(38)$ & $237.7 \pm 1.90(35)$ & $236.4 \pm 2.38(32)$ \\
9 & $253.1 \pm 2.43^{* *}(39)$ & $251.9 \pm 2.84(35)$ & $246.6 \pm 3.67(38)$ & $250.1 \pm 3.07(35)$ & $244.6 \pm 1.88^{*}(32)$ \\
12 & $270.4 \pm 2.03^{* *}(39)$ & $267.9 \pm 2.42(35)$ & $264.8 \pm 2.52(38)$ & $267.7 \pm 2.05(35)$ & $258.2 \pm 1.93 * *(32)$ \\
15 & $288.0 \pm 2.06^{* *}(39)$ & $284.5 \pm 2.64(35)$ & $280.7 \pm 3.14(38)$ & $286.5 \pm 2.39(35)$ & $274.8 \pm 2.30^{* *(32)}$ \\
18 & $326.2 \pm 2.48^{* *(39)}$ & $323.4 \pm 3.57(35)$ & $319.1 \pm 3.97(38)$ & $323.4 \pm 4.77(35)$ & $304.4 \pm 3.04 * *(32)$ \\
$21^{\text {c }}$ & $374.2 \pm 3.62^{* *(34)}$ & $365.6 \pm 5.14(30)$ & $361.0 \pm 6.19(33)$ & $365.2 \pm 6.95(31)$ & $335.6 \pm 4.63 * *(28)$
\end{tabular}

\section{Gestation Weight Change}

Gestation Day Interval

$\begin{array}{cccccc}6-9 & 15.70 \pm 1.35^{* *(39)} & 15.77 \pm 1.60(35) & 12.02 \pm 2.04(38) & 12.34 \pm 1.75(35) & 8.22 \pm 1.14 * *(32) \\ 9-12 & 17.31 \pm 1.17^{*}(39) & 15.98 \pm 1.19(35) & 18.24 \pm 1.61(38) & 17.64 \pm 1.71(35) & 13.66 \pm 0.64(32) \\ 12-15 & 17.61 \pm 0.68(39) & 16.68 \pm 0.67(35) & 15.91 \pm 1.45(38) & 18.73 \pm 0.87(35) & 16.53 \pm 0.72(32) \\ 15-18 & 38.18 \pm 0.95^{* *(39)} & 38.87 \pm 1.40(35) & 38.39 \pm 2.11(38) & 36.94 \pm 3.14(35) & 29.60 \pm 1.04 * *(32) \\ 18-21 & 48.47 \pm 1.29 * *(34) & 44.81 \pm 1.79(30) & 43.84 \pm 2.22(33) & 44.50 \pm 3.20(31) & 32.58 \pm 1.81 * *(28) \\ 6-21 & 137.2 \pm 2.96^{* *(34)} & 130.5 \pm 4.49(30) & 126.2 \pm 6.02(33) & 128.6 \pm 6.10(31) & 100.2 \pm 3.03 * *(28)\end{array}$

\section{Lactation Day}

$1 \quad 279.3 \pm 2.28 * *(34) \quad 278.4 \pm 2.68$ (29) $279.0 \pm 2.21(31) \quad 281.3 \pm 2.19$ (29) $254.3 \pm 2.06 * *(26)$

$4 \quad 294.4 \pm 2.53 * *(34) \quad 292.9 \pm 2.99(28)^{\mathrm{d}} \quad 292.2 \pm 2.36(29) \quad 292.7 \pm 2.60$ (29) $255.8 \pm 2.77 * *$ (26)

$7 \quad 312.7 \pm 2.43 * *(34) \quad 306.6 \pm 3.35(29) \quad 309.9 \pm 2.84(29) \quad 312.5 \pm 2.35$ (29) $254.2 \pm 3.07 * *(26)$

$14 \quad 317.4 \pm 2.29 * *(34) \quad 315.2 \pm 2.41$ (29) $317.3 \pm 3.07$ (29) $315.9 \pm 2.30$ (29) $238.7 \pm 3.84^{* *}(26)$

$21293.8 \pm 2.34 * *(34) \quad 294.9 \pm 2.52(29) \quad 293.6 \pm 2.77$ (29) $298.3 \pm 2.40$ (29) $230.1 \pm 3.79 * *(26)$

\section{Lactation Weight Change}

Lactation Day Interval

$$
\begin{array}{cccccc}
1-4 & 15.12 \pm 1.24 * *(34) & 14.23 \pm 0.99(28) & 12.53 \pm 1.44(29) & 11.34 \pm 1.75(29) & 1.53 \pm 1.81 * *(26) \\
4-7 & 18.27 \pm 1.19 * *(34) & 15.09 \pm 1.46(28) & 17.73 \pm 1.23(29) & 19.83 \pm 1.74(29) & -1.65 \pm 1.55^{* *}(26) \\
7-14 & 4.74 \pm 1.55^{* *(34)} & 8.58 \pm 2.22(29) & 7.42 \pm 2.56(29) & 3.40 \pm 1.93(29) & -15.5 \pm 2.67 * *(26) \\
14-21 & -23.6 \pm 1.40^{* *(34)} & -20.4 \pm 1.63(29) & -23.7 \pm 2.36(29) & -17.5 \pm 1.54 *(29) & -8.61 \pm 3.25 * *(26) \\
1-21 & 14.53 \pm 1.40^{* *}(34) & 16.47 \pm 1.44(29) & 13.93 \pm 2.36(29) & 17.02 \pm 2.80(29) & -24.2 \pm 3.02 * *(26)
\end{array}
$$

Statistical significance for an exposure group indicates a significant pairwise test compared to the vehicle control group.

Statistical significance for the vehicle control group indicates a significant trend test.

$*$ Statistically significant at $\mathrm{p} \leq 0.05 ; * * \mathrm{p} \leq 0.01$.

${ }^{a}$ Data are presented as mean \pm standard error (number of dams). Body weight data are presented in grams.

${ }^{b}$ Each exposure group was compared to the vehicle control group with the Williams test when a trend was present ( $p \leq 0.01$ from the Jonckheere trend test) or with the Dunnett test when no trend was present.

${ }^{\mathrm{c}}$ Decreased number of dams at gestation day (GD) 21 reflects animals removed at GD 18 for internal dose assessment.

${ }^{\mathrm{d}}$ One dam in the $300 \mathrm{ppm}$ group was removed as an outlier on lactation day 4. 
Table 4. Summary of Feed and Di(2-ethylhexyl) Phthalate Consumption by $F_{0}$ Female Rats during Gestation and Lactation in the Perinatal and Postweaning Two-year Feed Study

\begin{tabular}{|c|c|c|c|c|c|}
\hline Parameter $^{\mathbf{a}}$ & 0 ppm & $300 \mathrm{p}$ & 1,000 ppm & 3,000 ppm & 10,000 ppm \\
\hline \multicolumn{6}{|c|}{ Gestation Day Interval ${ }^{b, c}$} \\
\hline $6-9$ & $17.56 \pm 0.41^{* *}(39)$ & $17.64 \pm 0$ & $16.16 \pm$ & $16.75 \pm 0.58(35)$ & $13.97 \pm 0.22 * *(32)$ \\
\hline $9-12$ & $.64 \pm 0.2$ & $18.61 \pm 0$ & $18.33 \pm$ & $18.43 \pm 0.24(35)$ & 16.11 \\
\hline $12-15$ & $18.59 \pm 0.27(39)$ & $18.24 \pm 0.23(35)$ & $18.01 \pm 0.42(38)$ & $18.53 \pm 0.28(35)$ & $17.73 \pm 0.2$ \\
\hline $15-18$ & $20.41 \pm 0.36^{* *}(39)$ & $20.87 \pm 0.32(35)$ & $20.74 \pm 0.38(38)$ & $21.02 \pm 0.59(35)$ & $18.16 \pm 0.21^{* *}$ \\
\hline $18-21$ & $23.26 \pm 0.29 * *(34)$ & $22.59 \pm 0.37(30)$ & $22.89 \pm 0.41(33)$ & $23.41 \pm 0.57(31)$ & $19.29 \pm 0.29$ \\
\hline $6-21$ & $19.69 \pm 0.23 * *(34)$ & $19.45 \pm 0.26(30)$ & $19.17 \pm 0.29(33)$ & $19.50 \pm 0.30(31)$ & $17.03 \pm 0.19 * *(28)$ \\
\hline \multicolumn{6}{|c|}{ Lactation Day Interval ${ }^{b, c}$} \\
\hline $1-4$ & $35.54 \pm 0.71 * *(30)$ & $34.83 \pm 0.67(28)$ & $35.65 \pm 0.70(25)$ & $34.97 \pm 1.01(18)$ & $28.73 \pm 0.98$ \\
\hline $4-7$ & $47.91 \pm 0.79 * *$ & $45.83 \pm 0.78(29)$ & $47.26 \pm 0.98(29)$ & $47.81 \pm 1.29(29)$ & $30.00 \pm 0.14$ \\
\hline $7-10$ & $54.85 \pm 1.02 * *(34)$ & $51.92 \pm 0.98(29)$ & $53.85 \pm 1.04(28)$ & $53.65 \pm 1.49(29)$ & $31.38 \pm 0.75^{* *}(26)$ \\
\hline $10-14$ & $.36 \pm 0.98 * *$ & $63.12 \pm 0.76(29)$ & $61.30 \pm 1.42(29)$ & $61.74 \pm 1.70(29)$ & $33.36 \pm 0.92 * *$ \\
\hline $14-17$ & $62.05 \pm 1.13 * *(34)$ & $63.72 \pm 0.89(29)$ & $63.26 \pm 0.98(29)$ & $62.52 \pm 1.63(29)$ & $37.70 \pm 1.19 * *(26)$ \\
\hline $17-21$ & $86.25 \pm 1.28^{* *}(33)$ & $84.99 \pm 1.64(29)$ & $82.42 \pm 1.85(29)$ & $79.65 \pm 2.34 *(29)$ & $45.74 \pm 2.12 * *(26)$ \\
\hline $1-14$ & $51.01 \pm 0.80^{* *}(29)$ & $50.13 \pm 0.65(28)$ & $50.28 \pm 0.89(24)$ & $49.26 \pm 1.78(18)$ & $31.04 \pm 0.74 * *(23)$ \\
\hline
\end{tabular}

Chemical Intake (mg/kg/day) $)^{\mathrm{d}, \mathrm{e}}$

$\begin{array}{llllll}\text { GD 6-21 } & 0.00 \pm 0.00(34) & 20.58 \pm 0.16(30) & 68.11 \pm 0.71(33) & 205.6 \pm 2.10(31) & 625.6 \pm 6.23(28) \\ \text { LD 1-14 } & 0.00 \pm 0.00(29) & 49.40 \pm 0.52(27) & 165.5 \pm 2.77(24) & 482.1 \pm 15.93(18) & 1,244 \pm 25.58(23)\end{array}$

Statistical significance for an exposure group indicates a significant pairwise test compared to the vehicle control group.

Statistical significance for the vehicle control group indicates a significant trend test.

$*$ Statistically significant at $\mathrm{p} \leq 0.05 ; * \mathrm{p} \leq 0.01$.

$\mathrm{GD}=$ gestation day; $\mathrm{LD}=$ lactation day.

${ }^{\mathrm{a}}$ Data are presented as mean \pm standard error (number of dams).

${ }^{b}$ Feed consumption data are presented as grams/animal/day.

${ }^{c}$ Each exposure group was compared to the vehicle control group with the Shirley test when a trend was present ( $p \leq 0.01$ from the Jonckheere trend test) or with the Dunn test when no trend was present.

${ }^{\mathrm{d} C h e m i c a l}$ intake calculated as: ([exposure concentration $\times$ feed consumption]/[average body weight of day range]).

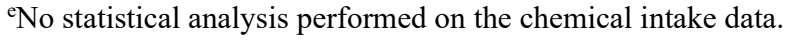

On postnatal day (PND) 1, total litter size and total live litter size of the 10,000 ppm group were significantly decreased, relative to the control group, corresponding to a reduction of approximately two pups per litter (Table 5). This litter effect corresponded to a significantly decreased number of live female offspring in 10,000 ppm DEHP-exposed litters. A significant decrease in the survival ratio (PND 5-21) of offspring exposed to 3,000 ppm was observed; however, this effect was not considered related to exposure because the survival ratio at $10,000 \mathrm{ppm}$ was not different from that of the control group. No other effect of DEHP exposure on offspring survival was observed during the preweaning intervals (PND 1-4, PND 5-21, or PND 1-21) (Table 5). 
Table 5. Summary of Mean Litter Size and Survival Ratio of $F_{1}$ Male and Female Rats during Lactation in the Perinatal and Postweaning Two-year Feed Study of Di(2-ethylhexyl) Phthalate

\begin{tabular}{|c|c|c|c|c|c|}
\hline Parameter & 0 ppm & 300 ppm & $1,000 \mathbf{p p m}^{\mathrm{a}}$ & $3,000 \mathrm{ppm}$ & $10,000 \mathrm{ppm}$ \\
\hline \multicolumn{6}{|l|}{ PND $1^{b, c}$} \\
\hline Total & $\begin{array}{c}12.59 \pm 0.38^{*} \\
(34)\end{array}$ & $\begin{array}{c}12.07 \pm 0.31 \\
(29)\end{array}$ & $\begin{array}{c}11.73 \pm 0.66 \\
(30)\end{array}$ & $\begin{array}{c}12.24 \pm 0.55 \\
(29)\end{array}$ & $\begin{array}{c}10.54 \pm 0.45^{* *} \\
(26)\end{array}$ \\
\hline Live & $\begin{array}{c}12.44 * * \pm 0.36 \\
(34)\end{array}$ & $\begin{array}{c}12.07 \pm 0.31 \\
(29)\end{array}$ & $\begin{array}{c}11.67 \pm 0.69 \\
(30)\end{array}$ & $\begin{array}{c}12.21 \pm 0.57 \\
(29)\end{array}$ & $\begin{array}{c}10.08 \pm 0.46^{* * *} \\
\text { (26) }\end{array}$ \\
\hline$\%$ Male per Litter & $\begin{array}{c}48.73 \pm 2.39 \\
\quad(34)\end{array}$ & $\begin{array}{c}46.87 \pm 2.02 \\
(29)\end{array}$ & $\begin{array}{c}51.95 \pm 2.89 \\
(28)\end{array}$ & $\begin{array}{c}49.06 \pm 2.80 \\
(29)\end{array}$ & $\begin{array}{c}54.83 \pm 2.93 \\
(26)\end{array}$ \\
\hline$\%$ Male $^{\mathrm{d}, \mathrm{e}}$ & $49(423)$ & $47(350)$ & $52(350)$ & $48(354)$ & $54(262)$ \\
\hline \multicolumn{6}{|l|}{ Male $^{b, c}$} \\
\hline PND 1 & $\begin{array}{l}6.12 \pm 0.38 \\
\quad(34)\end{array}$ & $\begin{array}{l}5.72 \pm 0.33 \\
\quad(29)\end{array}$ & $\begin{array}{l}6.03 \pm 0.48 \\
(30)\end{array}$ & $\begin{array}{l}5.90 \pm 0.42 \\
\quad(29)\end{array}$ & $\begin{array}{l}5.42 \pm 0.34 \\
\quad(26)\end{array}$ \\
\hline PND 4 & $\begin{array}{c}6.09 \pm 0.39 \\
\quad(34)\end{array}$ & $\begin{array}{l}5.76 \pm 0.32 \\
\quad(29)\end{array}$ & $\begin{array}{l}6.46 \pm 0.41 \\
\quad(28)\end{array}$ & $\begin{array}{l}5.86 \pm 0.41 \\
\quad(29)\end{array}$ & $\begin{array}{l}5.42 \pm 0.36 \\
\quad(26)\end{array}$ \\
\hline PND 21 & $\begin{array}{c}6.00 \pm 0.39 \\
(34)\end{array}$ & $\begin{array}{l}5.72 \pm 0.32 \\
(29)\end{array}$ & $\begin{array}{l}6.43 \pm 0.41 \\
(28)\end{array}$ & $\begin{array}{l}5.72 \pm 0.41 \\
\quad(29)\end{array}$ & $\begin{array}{l}5.31 \pm 0.35 \\
\quad(26)\end{array}$ \\
\hline \multicolumn{6}{|l|}{ Female $^{b, c}$} \\
\hline PND 1 & $\begin{array}{c}6.32 \pm 0.33^{*} \\
(34)\end{array}$ & $\begin{array}{c}6.34 \pm 0.24 \\
(29)\end{array}$ & $\begin{array}{l}5.63 \pm 0.48 \\
(30)\end{array}$ & $\begin{array}{c}6.31 \pm 0.47 \\
(29)\end{array}$ & $\begin{array}{c}4.65 \pm 0.35^{* *} \\
(26)\end{array}$ \\
\hline PND 4 & $\begin{array}{c}6.32 \pm 0.33^{* *} \\
(34)\end{array}$ & $\begin{array}{l}6.24 \pm 0.25 \\
(29)\end{array}$ & $\begin{array}{l}5.96 \pm 0.40 \\
\quad(28)\end{array}$ & $\begin{array}{l}6.28 \pm 0.48 \\
\quad(29)\end{array}$ & $\begin{array}{c}4.54 \pm 0.36^{* *} \\
(26)\end{array}$ \\
\hline PND 21 & $\begin{array}{c}6.26 \pm 0.33 * * \\
(34)\end{array}$ & $\begin{array}{c}6.14 \pm 0.25 \\
(29)\end{array}$ & $\begin{array}{l}5.93 \pm 0.41 \\
(28)\end{array}$ & $\begin{array}{l}5.97 \pm 0.49 \\
\quad(29)\end{array}$ & $\begin{array}{c}4.50 \pm 0.36 \text { ** } \\
\text { (26) }\end{array}$ \\
\hline \multicolumn{6}{|l|}{ Male and Female ${ }^{b, c}$} \\
\hline PND 4 & $\begin{array}{c}12.41 \pm 0.36^{* *} \\
(34)\end{array}$ & $\begin{array}{c}12.00 \pm 0.32 \\
(29)\end{array}$ & $\begin{array}{l}12.43 \pm 0.40 \\
\quad(28)\end{array}$ & $\begin{array}{c}12.14 \pm 0.57 \\
(29)\end{array}$ & $\begin{array}{c}9.96 \pm 0.44 * * \\
(26)\end{array}$ \\
\hline PND 7 & $\begin{array}{c}12.41 \pm 0.36^{* *} \\
(34)\end{array}$ & $\begin{array}{c}11.93 \pm 0.31 \\
(29)\end{array}$ & $\begin{array}{l}12.43 \pm 0.40 \\
\quad(28)\end{array}$ & $\begin{array}{c}11.97 \pm 0.57 \\
\quad(29)\end{array}$ & $\begin{array}{c}9.88 \pm 0.44^{* *} \\
(26)\end{array}$ \\
\hline PND 10 & $\begin{array}{c}12.38 \pm 0.36^{* *} \\
(34)\end{array}$ & $\begin{array}{c}11.90 \pm 0.31 \\
(29)\end{array}$ & $\begin{array}{c}12.43 \pm 0.40 \\
(28)\end{array}$ & $\begin{array}{c}11.83 \pm 0.59 \\
(29)\end{array}$ & $\begin{array}{c}9.88 \pm 0.44^{* *} \\
(26)\end{array}$ \\
\hline PND 14 & $\begin{array}{c}12.29 \pm 0.35^{* *} \\
(34)\end{array}$ & $\begin{array}{c}11.86 \pm 0.30 \\
(29)\end{array}$ & $\begin{array}{l}12.39 \pm 0.40 \\
\quad(28)\end{array}$ & $\begin{array}{c}11.69 \pm 0.59 \\
(29)\end{array}$ & $\begin{array}{c}9.81 \pm 0.43 * * \\
(26)\end{array}$ \\
\hline PND 17 & $\begin{array}{c}12.29 \pm 0.35^{* *} \\
(34)\end{array}$ & $\begin{array}{c}11.86 \pm 0.30 \\
(29)\end{array}$ & $\begin{array}{c}12.36 \pm 0.39 \\
(28)\end{array}$ & $\begin{array}{c}11.69 \pm 0.59 \\
(29)\end{array}$ & $\begin{array}{c}9.81 \pm 0.43^{* *} \\
(26)\end{array}$ \\
\hline PND 21 & $\begin{array}{c}12.26 \pm 0.35^{* *} \\
(34)\end{array}$ & $\begin{array}{c}11.86 \pm 0.30 \\
(29)\end{array}$ & $\begin{array}{c}12.36 \pm 0.39 \\
(28)\end{array}$ & $\begin{array}{c}11.69 \pm 0.59 \\
(29)\end{array}$ & $\begin{array}{c}9.81 \pm 0.43 * * \\
(26)\end{array}$ \\
\hline \multicolumn{6}{|l|}{ Survival per Litter } \\
\hline Total Dead: PND 1-4 ${ }^{\mathrm{f}}$ & $6(34)$ & $2(29)$ & $3(30)$ & $3(29)$ & $15(26)$ \\
\hline Total Dead: PND 5-21 ${ }^{\mathrm{f}}$ & $5(34)$ & $4(29)$ & $2(28)$ & $13(29)$ & $4(26)$ \\
\hline Dead: PND 1-4,c,g & $\begin{array}{c}0.176 \pm 0.079 \\
(34)\end{array}$ & $\begin{array}{c}0.069 \pm 0.048 \\
\text { (29) }\end{array}$ & $\begin{array}{c}0.100 \pm 0.056 \\
(30)\end{array}$ & $\begin{array}{c}0.103 \pm 0.058 \\
\text { (29) }\end{array}$ & $\begin{array}{c}0.577 \pm 0.294 \\
(26)\end{array}$ \\
\hline
\end{tabular}




\begin{tabular}{|c|c|c|c|c|c|}
\hline Parameter & O ppm & 300 ppm & $1,000 \mathbf{p p m}^{\mathrm{a}}$ & 3,000 ppm & 10,000 ppm \\
\hline Dead: PND 5-21 b,c,g & $\begin{array}{c}0.147 \pm 0.096 \\
\text { (34) }\end{array}$ & $\begin{array}{c}0.138 \pm 0.108 \\
\text { (29) }\end{array}$ & $\begin{array}{c}0.071 \pm 0.050 \\
(28)\end{array}$ & $\begin{array}{c}0.448 \pm 0.183 * \\
\text { (29) }\end{array}$ & $\begin{array}{c}0.154 \pm 0.091 \\
(26)\end{array}$ \\
\hline Dead: PND 1-21 $11^{\mathrm{b}, \mathrm{c}, \mathrm{g}}$ & $\begin{array}{c}0.324 \pm 0.151 \\
(34)\end{array}$ & $\begin{array}{c}0.207 \pm 0.115 \\
\text { (29) }\end{array}$ & $\begin{array}{c}0.167 \pm 0.069 \\
(30)\end{array}$ & $\begin{array}{c}0.552 \pm 0.214 \\
(29)\end{array}$ & $\begin{array}{c}0.731 \pm 0.326 \\
(26)\end{array}$ \\
\hline Survival Ratio: PND 1-4 & $\begin{array}{c}0.998 \pm 0.002 \\
(34)\end{array}$ & $\begin{array}{c}0.994 \pm 0.004 \\
(29)\end{array}$ & $\begin{array}{c}0.997 \pm 0.003 \\
\quad(28)\end{array}$ & $\begin{array}{l}0.994 \pm 0.004 \\
\quad(29)\end{array}$ & $\begin{array}{c}0.990 \pm 0.005 \\
(26)\end{array}$ \\
\hline Survival Ratio: PND 5-21 & $\begin{array}{c}0.990 \pm 0.007 \\
(34)\end{array}$ & $\begin{array}{c}0.990 \pm 0.008 \\
(29)\end{array}$ & $\begin{array}{c}0.995 \pm 0.003 \\
(28)\end{array}$ & $\begin{array}{c}0.962 \pm 0.015 * \\
\text { (29) }\end{array}$ & $\begin{array}{c}0.986 \pm 0.008 \\
(26)\end{array}$ \\
\hline Survival Ratio: PND 1-21, b,c,j & $\begin{array}{c}0.987 \pm 0.007 \\
\text { (34) }\end{array}$ & $\begin{array}{c}0.984 \pm 0.008 \\
\text { (29) }\end{array}$ & $\begin{array}{c}0.992 \pm 0.004 \\
\text { (28) }\end{array}$ & $\begin{array}{c}0.958 \pm 0.016 \\
(29)\end{array}$ & $\begin{array}{c}0.977 \pm 0.010 \\
(26)\end{array}$ \\
\hline
\end{tabular}

Statistical significance for an exposure group indicates a significant pairwise test compared to the vehicle control group. Statistical significance for the vehicle control group indicates a significant trend test.

*Statistically significant at $\mathrm{p} \leq 0.05 ; * * \mathrm{p} \leq 0.01$.

PND $=$ postnatal day.

${ }^{a}$ One dam in the $1,000 \mathrm{ppm}$ group was not included in any endpoint calculations due to differing pup counts on PND 1 and PND 4. Two additional dams in the 1,000 ppm group produced single pups that died on PND 1; these dams were only included in the PND 1 calculations.

${ }^{b}$ Each exposure group was compared to the vehicle control group with the Shirley test when a trend was present ( $p \leq 0.01$ from the Jonckheere trend test) or with the Dunn test when no trend was present.

${ }^{c}$ Data are presented as mean \pm standard error (number of litters).

${ }^{\mathrm{d}}[100 \times($ number of live males in exposure group)/(number of live males and females in exposure group)] (number of pups).

${ }^{\mathrm{e}}$ No statistics performed on this endpoint.

${ }^{\mathrm{f}}$ Total number of dead pups in exposure group (number of litters).

gNumber dead per litter.

hurvival per litter: Number of live pups on PND 4/number of live pups on PND 1.

iSurvival per litter: Number of live pups on PND 21/number of live pups on PND 5.

jSurvival per litter: Number of live pups on PND 21/number of live pups on PND 1.

Decreased PND 1 pup mean body weights were observed in male, female, and combined (male + female) offspring in 3,000 and 10,000 ppm DEHP litters (Table 6). PND 1 pup mean body weights (male, female, and combined) were significantly decreased by approximately $4 \%$ in the 3,000 ppm group, and by $15 \%, 12 \%$, and $13 \%$ in males, females, and combined offspring in the 10,000 ppm group, respectively, relative to control animals. At weaning (PND 21), male and female pup mean body weights in the 1,000 and 3,000 ppm DEHP groups were significantly decreased approximately $6 \%$ compared to control animals. Severe effects on growth were evident in male and female offspring exposed to 10,000 ppm DEHP. At weaning (PND 21), mean body weights of male and female pups in the 10,000 ppm group were significantly decreased approximately 55\% and 53\% relative to those of control animals, respectively (Table 6). Significantly decreased mean body weights in 10,000 ppm pups were attributed to reduced body weight gain throughout the preweaning interval. Pup survival was not reduced, and there were no exposure-related clinical observations. Offspring from the 10,000 ppm group were therefore continued on study. 
Table 6. Summary of Preweaning $F_{1}$ Male and Female Rat Pup Mean Body Weights Following Perinatal Exposure to Di(2-ethylhexyl) Phthalate

\begin{tabular}{|c|c|c|c|c|c|}
\hline Parameter $^{\mathrm{a}}$ & 0 ppm & 300 ppm & 1,000 ppm $^{\mathrm{b}}$ & $3,000 \mathrm{ppm}$ & 10,000 ppm $^{\mathrm{c}}$ \\
\hline \multicolumn{6}{|l|}{ Male (g) } \\
\hline PND $1^{\mathrm{d}, \mathrm{e}, \mathrm{f}}$ & $7.45 \pm 0.09 * *$ & $7.59 \pm 0.08(29)$ & $7.43 \pm 0.10(28)$ & $7.16 \pm 0.10^{*}(29)$ & $6.34 \pm 0.09^{* *}(26)$ \\
\hline PND 4, ${ }^{\mathrm{g}, \mathrm{h}}$ & $\begin{array}{l}10.64 \pm 0.13^{* *} \\
(207 / 34)\end{array}$ & $\begin{array}{c}10.51 \pm 0.19 \\
(167 / 29)\end{array}$ & $\begin{array}{c}10.72 \pm 0.16 \\
(181 / 28)\end{array}$ & $\begin{array}{c}10.34 \pm 0.18 \\
(170 / 29)\end{array}$ & $\begin{array}{l}11.13 \pm 0.20^{* *} \\
\quad(140 / 26)\end{array}$ \\
\hline PND $7^{\mathrm{g}, \mathrm{h}}$ & $\begin{array}{c}15.12 \pm 0.20^{* *} \\
(207 / 34)\end{array}$ & $\begin{array}{c}14.69 \pm 0.28 \\
(166 / 29)\end{array}$ & $\begin{array}{c}14.71 \pm 0.27 \\
(181 / 28)\end{array}$ & $\begin{array}{c}14.60 \pm 0.23 \\
(168 / 29)\end{array}$ & $\begin{array}{c}11.13 \pm 0.20^{* * *} \\
(140 / 26)\end{array}$ \\
\hline PND $14^{\mathrm{g}, \mathrm{h}}$ & $\begin{array}{l}29.19 \pm 0.30^{* *} \\
\quad(205 / 34)\end{array}$ & $\begin{array}{l}28.44 \pm 0.42 \\
(166 / 29)\end{array}$ & $\begin{array}{c}27.80 \pm 0.47^{*} \\
(181 / 28)\end{array}$ & $\begin{array}{l}28.49 \pm 0.44 \\
(166 / 29)\end{array}$ & $\begin{array}{c}17.24 \pm 0.36^{* *} \\
(138 / 26)\end{array}$ \\
\hline PND $21^{\mathrm{g}, \mathrm{h}}$ & $\begin{array}{c}48.65 \pm 0.59^{* *} \\
(204 / 34)\end{array}$ & $\begin{array}{c}47.05 \pm 0.72 \\
(166 / 29)\end{array}$ & $\begin{array}{c}45.66 \pm 0.79^{*} \\
(180 / 28)\end{array}$ & $\begin{array}{c}45.53 \pm 0.83^{*} \\
(166 / 29)\end{array}$ & $\begin{array}{c}21.82 \pm 0.84^{* *} \\
\quad(138 / 26)\end{array}$ \\
\hline \multicolumn{6}{|l|}{ Female (g) } \\
\hline PND $1^{\mathrm{d}, \mathrm{e}, \mathrm{f}}$ & $7.01 \pm 0.06^{* *}$ & $7.17 \pm 0.07(29)$ & $7.02 \pm 0.09(28)$ & $6.76 \pm 0.09 *(29)$ & $6.19 \pm 0.13 * *$ \\
\hline PND $4^{\mathrm{g}, \mathrm{h}}$ & $\begin{array}{l}10.09 \pm 0.11^{* *} \\
(215 / 34)\end{array}$ & $\begin{array}{c}10.07 \pm 0.13 \\
(181 / 29)\end{array}$ & $\begin{array}{c}10.18 \pm 0.17 \\
(167 / 28)\end{array}$ & $\begin{array}{c}9.67 \pm 0.18 \\
(182 / 29)\end{array}$ & $\begin{array}{c}8.35 \pm 0.21 * * \\
(117 / 26)\end{array}$ \\
\hline PND $7^{\mathrm{g}, \mathrm{h}}$ & $\begin{array}{c}14.25 \pm 0.19^{* *} \\
(215 / 34)\end{array}$ & $\begin{array}{c}13.97 \pm 0.23 \\
(180 / 29)\end{array}$ & $\begin{array}{c}13.92 \pm 0.26 \\
(167 / 28)\end{array}$ & $\begin{array}{c}13.50 \pm 0.28 \\
(179 / 29)\end{array}$ & $\begin{array}{c}10.97 \pm 0.26^{* * *} \\
(117 / 26)\end{array}$ \\
\hline PND 14, & $\begin{array}{l}27.97 \pm 0.30^{* *} \\
(213 / 34)\end{array}$ & $\begin{array}{l}27.41 \pm 0.28 \\
(178 / 29)\end{array}$ & $\begin{array}{l}26.57 \pm 0.45^{*} \\
(166 / 28)\end{array}$ & $\begin{array}{c}27.19 \pm 0.44 \\
(173 / 29)\end{array}$ & $\begin{array}{l}16.91 \pm 0.44^{* *} \\
\quad(117 / 26)\end{array}$ \\
\hline PND $21^{g, h}$ & $\begin{array}{l}45.85 \pm 0.52 * * \\
\quad(213 / 34)\end{array}$ & $\begin{array}{c}44.84 \pm 0.56 \\
(178 / 29)\end{array}$ & $\begin{array}{c}43.27 \pm 0.78^{*} \\
(166 / 28)\end{array}$ & $\begin{array}{l}42.91 \pm 0.84^{*} \\
(173 / 29)\end{array}$ & $\begin{array}{l}21.64 \pm 0.91^{* *} \\
(117 / 26)\end{array}$ \\
\hline \multicolumn{6}{|c|}{ Male and Female (g) } \\
\hline PND $1^{\mathrm{d}, \mathrm{e}, \mathrm{f}}$ & $7.20 \pm 0.07 * *$ & $7.35 \pm 0.07(29)$ & $7.22 \pm 0.09(28)$ & $6.96 \pm 0.09 *(29)$ & $6.29 \pm 0.10^{* *}(26)$ \\
\hline PND 4, ${ }^{g, h}$ & $\begin{array}{l}10.33 \pm 0.11^{* *} \\
\quad(422 / 34)\end{array}$ & $\begin{array}{c}10.28 \pm 0.14 \\
(348 / 29)\end{array}$ & $\begin{array}{c}10.47 \pm 0.16 \\
(348 / 28)\end{array}$ & $\begin{array}{c}10.00 \pm 0.17 \\
(352 / 29)\end{array}$ & $\begin{array}{l}8.51 \pm 0.16^{* *} \\
(258 / 26)\end{array}$ \\
\hline PND $7^{\mathrm{g}, \mathrm{h}}$ & $\begin{array}{c}14.66 \pm 0.19 * * \\
(422 / 34)\end{array}$ & $\begin{array}{c}14.32 \pm 0.23 \\
(346 / 29)\end{array}$ & $\begin{array}{c}14.36 \pm 0.26 \\
(348 / 28)\end{array}$ & $\begin{array}{c}14.05 \pm 0.22 \\
(347 / 29)\end{array}$ & $\begin{array}{c}11.10 \pm 0.20^{* *} \\
(257 / 26)\end{array}$ \\
\hline PND 14, & $\begin{array}{l}28.52 \pm 0.28^{* *} \\
\quad(418 / 34)\end{array}$ & $\begin{array}{l}27.91 \pm 0.32 \\
(344 / 29)\end{array}$ & $\begin{array}{l}27.23 \pm 0.45^{*} \\
\quad(347 / 28)\end{array}$ & $\begin{array}{l}27.74 \pm 0.40 \\
(339 / 29)\end{array}$ & $\begin{array}{l}17.14 \pm 0.39 * * \\
(255 / 26)\end{array}$ \\
\hline PND $21^{g, h}$ & $\begin{array}{c}47.18 \pm 0.51^{* *} \\
(417 / 34)\end{array}$ & $\begin{array}{c}45.90 \pm 0.60 \\
(344 / 29)\end{array}$ & $\begin{array}{c}44.51 \pm 0.77 * \\
(346 / 28)\end{array}$ & $\begin{array}{l}44.08 \pm 0.77 * * \\
(339 / 29)\end{array}$ & $\begin{array}{c}21.79 \pm 0.86^{* * *} \\
(255 / 26)\end{array}$ \\
\hline
\end{tabular}

Statistical significance for an exposure group indicates a significant pairwise test compared to the vehicle control group.

Statistical significance for the vehicle control group indicates a significant trend test.

*Statistically significant at $\mathrm{p} \leq 0.05 ; * * \mathrm{p} \leq 0.01$.

PND = postnatal day.

aStatistical analysis performed using mixed models with random litter effect for both trend and pairwise tests, using the Dunnett-Hsu adjustment for multiple comparisons (unless otherwise noted).

${ }^{\mathrm{b}}$ All pups from one dam in the 1,000 ppm group were excluded from all body weight calculations due to that dam having differing litter size counts from PND 1 to PND 4. Additionally, one pup was removed from all body weight calculations due to litter misclassification.

${ }^{\mathrm{c}}$ Two pups from two dams in the 10,000 ppm group were not included in the PND 4 body weight calculations. One pup was removed as an outlier and the other was excluded due to the pup dying on PND 4.

${ }^{\mathrm{d}}$ Data are presented as mean \pm standard error (number of litters).

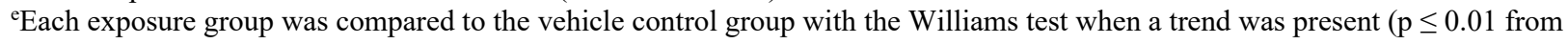
the Jonckheere trend test) or with the Dunnett test when no trend was present.

${ }^{\mathrm{f}}$ Total pup weight at PND 1 divided by number of live pups at PND 1.

gData are presented as mean of litter means \pm standard error (number of pups/number of litters).

hIndividual pup weights first adjusted for live litter size on PND 1. 
Concentrations of the DEHP metabolite mono(2-ethylhexyl) phthalate (MEHP) were determined in maternal plasma, amniotic fluid, and fetal tissues collected on GD 18 (Table 7) using validated analytical methods (Appendix E). MEHP concentrations increased proportionally to exposure concentration in dam plasma in groups exposed to lower dietary DEHP concentrations (300 and $1,000 \mathrm{ppm})$. However, at higher dietary concentrations $(3,000$ and 10,000 ppm), the increase was greater than proportional to exposure concentration, despite proportional increases in chemical consumption, suggesting potential saturation of clearance pathways of MEHP (Figure 2). MEHP was measured in amniotic fluid and fetuses demonstrating transfer of MEHP across the placental barrier and exposure of the developing conceptus. The concentrations in fetuses were approximately $18 \%-28 \%$ of the plasma concentrations in dams, suggesting gestational transfer was moderate for MEHP, but the rate of transfer of other metabolites was not measured. MEHP was detected in amniotic fluid and fetus samples from control animals, whereas control dam plasma concentrations were below the limit of detection.

Table 7. Summary of Internal Dose Data for Rats in the Perinatal and Postweaning Two-year Feed Study of Di(2-ethylhexyl) Phthalate

\begin{tabular}{lccccc}
\hline & $\mathbf{0 ~ p p m}$ & $\mathbf{3 0 0} \mathbf{~ p p m}$ & $\mathbf{1 , 0 0 0} \mathbf{~ p p m}$ & $\mathbf{3 , 0 0 0} \mathbf{~ p p m}$ & $\mathbf{1 0 , 0 0 0} \mathbf{~ p p m}$ \\
\hline $\mathbf{n}$ & 5 & 5 & 5 & $4^{\mathrm{a}}$ & $4^{\mathrm{b}}$ \\
\hline \multicolumn{7}{l}{$\begin{array}{l}\text { Mono(2-ethylhexyl) Phthalate Concentration } \\
\text { Gestation Day } 18\end{array}$} & & & & \\
Dam plasma (ng/mL) & $\mathrm{BD}^{\mathrm{e}}$ & $630.2 \pm 84.7$ & $2,000.0 \pm 156.9$ & $8,950.0 \pm 768.4$ & $39,800.0 \pm 4,192.9$ \\
Amniotic fluid (ng/mL) & $45.6 \pm 2.0^{* *}$ & $73.4 \pm 2.7^{* *}$ & $123.0 \pm 6.2^{* *}$ & $456.8 \pm 10.6^{* *}$ & $1,685.0 \pm 156.0^{* *}$ \\
$\quad$ Fetuses (ng/g) & $53.2 \pm 7.7^{\mathrm{f} * *}$ & $178.8 \pm 22.2^{* *}$ & $383.4 \pm 18.8^{* *}$ & $1,580.0 \pm 105.4^{* *}$ & $8,295.0 \pm 813.3^{* *}$ \\
\hline
\end{tabular}

Statistical significance for an exposure group indicates a significant pairwise test compared to the vehicle control group.

Statistical significance for the vehicle control group indicates a significant trend test.

$* *$ Statistically significant at $\mathrm{p} \leq 0.01$.

$\mathrm{BD}=$ below detection; group did not have over $20 \%$ of its values above the limit of detection.

${ }^{\mathrm{a}}$ One female in the 3,000 ppm group was found not to be pregnant and was replaced with an additional dam. The replacement dam was also found not to be pregnant, and another replacement dam was not selected.

${ }^{\mathrm{b}}$ Two females in the 10,000 ppm group were found not to be pregnant; one replacement dam was selected and added for analysis. ${ }^{\mathrm{c}}$ Data are presented as mean \pm standard error.

If over $20 \%$ of the animals in a group were above the limit of detection (LOD), one-half of the LOD value was substituted for values below the LOD. LOD for dam plasma $=5 \mathrm{ng} / \mathrm{mL}$; LOD for amniotic fluid $=12 \mathrm{ng} / \mathrm{mL}$; LOD for fetuses $=10 \mathrm{ng} / \mathrm{g}$.

${ }^{e}$ When the vehicle control group did not have over $20 \%$ of its values above the LOD, no mean or standard error were calculated and no statistical analysis was performed.

fStatistical analysis performed by the Jonckheere (trend) and the Shirley or Dunn (pairwise) tests.

\section{Postweaning Phase}

Overall, survival at study termination of males and females in exposed groups was commensurate with control groups (Table 8; Figure 3). However, 6 to 7 rats per sex in the $10,000 \mathrm{ppm}$ group died postweaning during the first two weeks of the study period. All but one of these early losses likely resulted from the severely reduced body weights of those animals. 
Table 8. Summary of Survival of Male and Female Rats in the Perinatal and Postweaning Two-year Feed Study of Di(2-ethylhexyl) Phthalate

\begin{tabular}{|c|c|c|c|c|c|}
\hline & O ppm & 300 ppm & 1,000 ppm & 3,000 ppm & 10,000 ppm \\
\hline \multicolumn{6}{|l|}{ Male } \\
\hline Animals Initially in Study & 50 & $49^{\mathrm{a}}$ & 50 & 50 & 50 \\
\hline Moribund & 12 & 9 & 4 & 8 & 7 \\
\hline Natural Deaths & 13 & 7 & 6 & 7 & 14 \\
\hline Animals Surviving to Study Termination & $25^{\mathrm{b}}$ & 33 & $40^{\mathrm{b}}$ & 35 & $29^{\mathrm{b}}$ \\
\hline $\begin{array}{l}\text { Percent Probability of Survival at End of } \\
\text { Study }^{c}\end{array}$ & 50.0 & 67.3 & 80.0 & 70.0 & 58.0 \\
\hline Mean Survival (Days) ${ }^{\mathrm{d}}$ & 648 & 683 & 713 & 694 & 555 \\
\hline Survival Analysis ${ }^{\mathrm{e}}$ & $\mathrm{p}=0.171$ & $\mathrm{p}=0.118 \mathrm{~N}$ & $\mathrm{p}=0.004 \mathrm{~N}$ & $\mathrm{p}=0.059 \mathrm{~N}$ & $\mathrm{p}=0.938 \mathrm{~N}$ \\
\hline \multicolumn{6}{|l|}{ Female } \\
\hline Animals Initially in Study & 50 & 50 & 50 & 50 & 50 \\
\hline Moribund & 13 & 9 & 9 & 7 & 12 \\
\hline Natural Deaths & 6 & 9 & 7 & 9 & 11 \\
\hline Animals Surviving to Study Termination & 31 & 32 & 34 & $34^{\mathrm{f}}$ & 27 \\
\hline $\begin{array}{l}\text { Percent Probability of Survival at End of } \\
\text { Study }\end{array}$ & 62.0 & 64.0 & 68.0 & 68.0 & 54.0 \\
\hline Mean Survival (Days) & 684 & 671 & 679 & 681 & 594 \\
\hline Survival Analysis & $\mathrm{p}=0.083$ & $\mathrm{p}=0.995 \mathrm{~N}$ & $\mathrm{p}=0.693 \mathrm{~N}$ & $\mathrm{p}=0.667 \mathrm{~N}$ & $\mathrm{p}=0.185$ \\
\hline
\end{tabular}

${ }^{a}$ One pup was mis-sexed at the beginning of the study and was removed.

bIncludes one animal that died naturally during the last week of the study.

'Kaplan-Meier determinations.

${ }^{\mathrm{d}}$ Mean of litter means of all deaths (uncensored, censored, and study termination).

'The result of the Cox proportional hazards trend test is in the vehicle control column, and the results of the Cox proportional hazards pairwise comparisons with the vehicle control group are in the exposed group columns. A negative trend or lower mortality in an exposure group is indicated by $\mathrm{N}$.

fIncludes one animal that died naturally and one animal that was euthanized moribund during the last week of the study. 

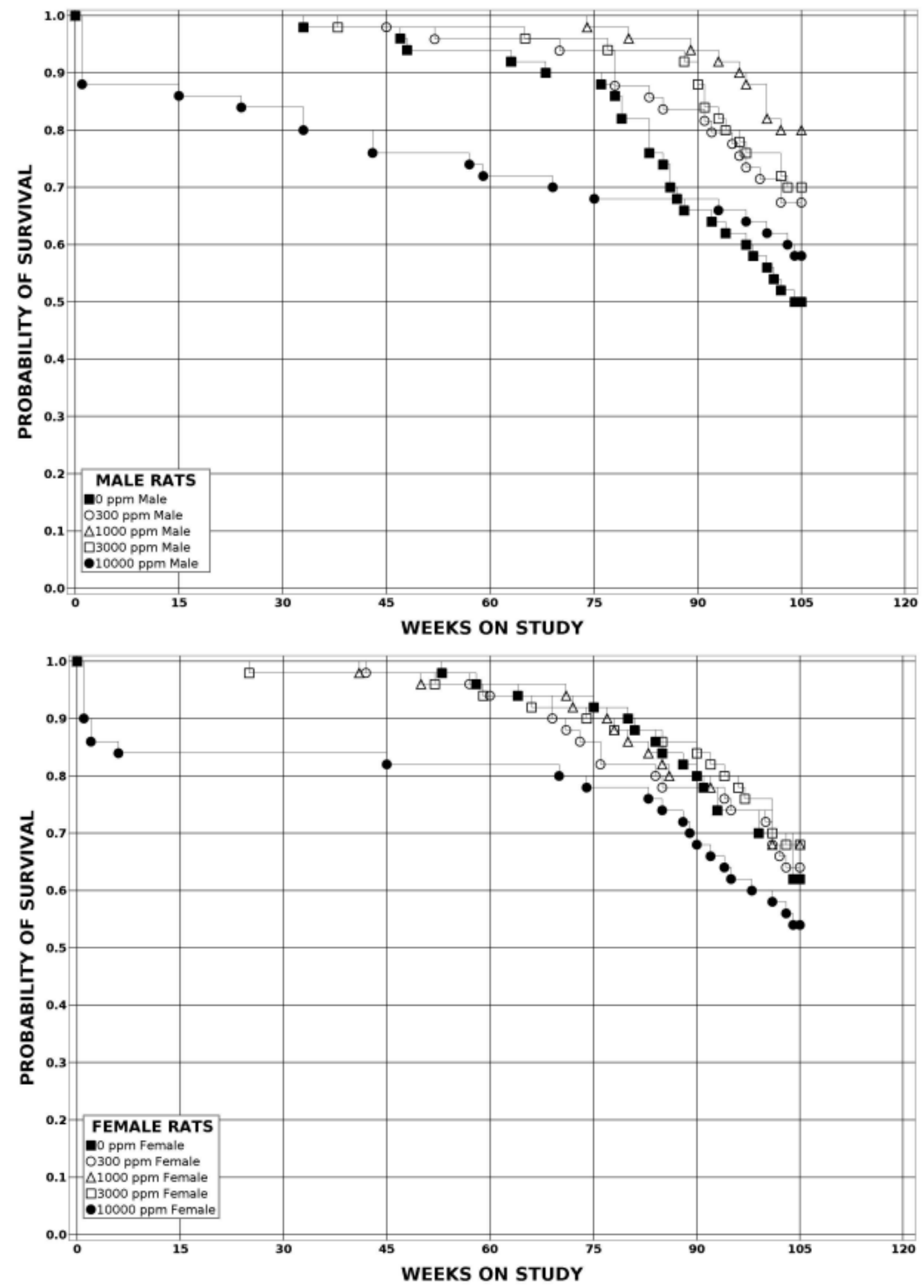

Figure 3. Kaplan-Meier Survival Curves for Rats Exposed to Di(2-ethylhexyl) Phthalate in the Perinatal and Postweaning Two-year Feed Study 
At study termination, group mean body weights for male and female rats in the $300,1,000$, and 3,000 ppm DEHP groups were within 10\% of their respective control groups (Table 9, Table 10; Figure 4). During the first 3 (females) or 6 (males) weeks on study postweaning, mean body weights of rats in the $10,000 \mathrm{ppm}$ groups were approximately half that of their respective control groups. Following week 3 (females) or week 6 (males) on study, however, mean body weights of those male and female rats exhibited a moderate recovery, attaining maximum group mean body weights that were $28 \%$ and $17 \%$ lower, respectively, relative to control animals during the chronic study period. The terminal mean body weights of 10,000 ppm males and females were $30 \%$ and $32 \%$ lower than those of control animals, respectively. Lower body weights observed in the $10,000 \mathrm{ppm}$ groups at study termination were attributed to reduced body weight gain observed over the duration of the study.

Feed consumption by male and female rats in the 300, 1,000, and 3,000 ppm DEHP groups was commensurate to that of the control groups throughout the study (Table 11, Table 12; Appendix H). Feed consumption was generally lower in the $10,000 \mathrm{ppm}$ male and female rat groups with the largest difference directly following weaning. This finding was restricted to the early time interval and likely resulted from the significantly reduced body size of animals in the highest exposure group. Dietary concentrations of 300,1,000, 3,000, and 10,000 ppm resulted in average daily doses of approximately $18,58,189$, and $678 \mathrm{mg} / \mathrm{kg} /$ day for males and 18, 62, 196, and $772 \mathrm{mg} / \mathrm{kg} /$ day for females (Appendix H).

No exposure-related clinical findings were observed in any of the exposed groups (Appendix $\mathrm{H}$ ). 
Table 9. Summary of Survival and Mean Body Weights of Male Rats in the Perinatal and Postweaning Two-year Feed Study of Di(2-ethylhexyl) Phthalate

\begin{tabular}{|c|c|c|c|c|c|c|c|c|c|c|c|c|c|c|}
\hline \multirow[b]{2}{*}{$\begin{array}{l}\text { Study } \\
\text { Day }^{\mathbf{a}}\end{array}$} & \multicolumn{2}{|c|}{ O ppm } & \multicolumn{3}{|c|}{300 ppm } & \multicolumn{3}{|c|}{1,000 ppm } & \multicolumn{3}{|c|}{$3,000 \mathrm{ppm}$} & \multicolumn{3}{|c|}{10,000 ppm } \\
\hline & $\begin{array}{l}\text { Av. } \\
\text { Wt. } \\
(\mathbf{g})^{\mathbf{b}}\end{array}$ & $\begin{array}{l}\text { No. of } \\
\text { Litters }\end{array}$ & $\begin{array}{l}\text { Av. } \\
\text { Wt. } \\
\text { (g) }\end{array}$ & $\begin{array}{c}\text { Wt. } \\
\text { (\% of } \\
\text { Controls) }\end{array}$ & $\begin{array}{l}\text { No. of } \\
\text { Litters }\end{array}$ & $\begin{array}{l}\text { Av. } \\
\text { Wt. } \\
\text { (g) }\end{array}$ & $\begin{array}{c}\text { Wt. } \\
\text { (\% of } \\
\text { Controls) } \\
\end{array}$ & $\begin{array}{l}\text { No. of } \\
\text { Litters }\end{array}$ & $\begin{array}{l}\text { Av. } \\
\text { Wt. } \\
\text { (g) }\end{array}$ & $\begin{array}{c}\text { Wt. } \\
\text { (\% of } \\
\text { Controls) }\end{array}$ & $\begin{array}{l}\text { No. of } \\
\text { Litters }\end{array}$ & $\begin{array}{l}\text { Av. } \\
\text { Wt. } \\
\text { (g) }\end{array}$ & $\begin{array}{c}\text { Wt. } \\
\text { (\% of } \\
\text { Controls) }\end{array}$ & $\begin{array}{l}\text { No. of } \\
\text { Litters }\end{array}$ \\
\hline 1 & 50.8 & 25 & 50.6 & 99.5 & 25 & 49.2 & 96.7 & 25 & 48.9 & 96.1 & 25 & 27.5 & 54.1 & 25 \\
\hline 8 & 82.1 & 25 & 80.6 & 98.1 & 25 & 78.5 & 95.6 & 25 & 76.6 & 93.3 & 25 & 35.7 & 43.4 & 23 \\
\hline 15 & 127.7 & 25 & 121.3 & 95.0 & 25 & 119.0 & 93.2 & 25 & 117.6 & 92.1 & 25 & 53.5 & 41.9 & 23 \\
\hline 22 & 175.0 & 25 & 168.2 & 96.1 & 25 & 165.9 & 94.8 & 25 & 164.6 & 94.1 & 25 & 75.6 & 43.2 & 23 \\
\hline 29 & 217.7 & 25 & 214.4 & 98.5 & 25 & 212.6 & 97.7 & 25 & 212.2 & 97.5 & 25 & 101.5 & 46.6 & 23 \\
\hline 36 & 262.2 & 25 & 257.7 & 98.3 & 25 & 255.3 & 97.4 & 25 & 257.0 & 98.0 & 25 & 130.3 & 49.7 & 23 \\
\hline 43 & 293.6 & 25 & 290.5 & 99.0 & 25 & 285.6 & 97.3 & 25 & 287.4 & 97.9 & 25 & 158.0 & 53.8 & 23 \\
\hline 50 & 316.3 & 25 & 314.8 & 99.5 & 25 & 310.3 & 98.1 & 25 & 310.6 & 98.2 & 25 & 186.1 & 58.8 & 23 \\
\hline 57 & 333.3 & 25 & 334.7 & 100.4 & 25 & 330.6 & 99.2 & 25 & 327.7 & 98.3 & 25 & 208.2 & 62.5 & 23 \\
\hline 64 & 350.8 & 25 & 351.4 & 100.2 & 25 & 347.2 & 98.9 & 25 & 342.9 & 97.7 & 25 & 225.4 & 64.2 & 23 \\
\hline 71 & 364.6 & 25 & 365.0 & 100.1 & 25 & 357.4 & 98.0 & 25 & 352.7 & 96.7 & 25 & 239.9 & 65.8 & 23 \\
\hline 78 & 375.8 & 25 & 375.0 & 99.8 & 25 & 368.8 & 98.1 & 25 & 364.4 & 97.0 & 25 & 251.2 & 66.8 & 23 \\
\hline 85 & 386.3 & 25 & 383.2 & 99.2 & 25 & 379.7 & 98.3 & 25 & 375.0 & 97.1 & 25 & 263.2 & 68.1 & 23 \\
\hline 92 & 396.8 & 25 & 391.0 & 98.5 & 25 & 386.4 & 97.4 & 25 & 381.8 & 96.2 & 25 & 271.6 & 68.4 & 23 \\
\hline 120 & 427.2 & 25 & 412.5 & 96.6 & 25 & 409.2 & 95.8 & 25 & 398.4 & 93.3 & 25 & 288.7 & 67.6 & 22 \\
\hline 148 & 451.3 & 25 & 443.2 & 98.2 & 25 & 436.5 & 96.7 & 25 & 420.6 & 93.2 & 25 & 315.8 & 70.0 & 22 \\
\hline 176 & 471.7 & 25 & 464.9 & 98.6 & 25 & 454.3 & 96.3 & 25 & 440.4 & 93.4 & 25 & 332.7 & 70.5 & 22 \\
\hline 204 & 483.6 & 25 & 473.0 & 97.8 & 25 & 471.4 & 97.5 & 25 & 454.8 & 94.0 & 25 & 345.8 & 71.5 & 22 \\
\hline 232 & 498.1 & 25 & 484.4 & 97.3 & 25 & 484.3 & 97.2 & 25 & 458.4 & 92.0 & 25 & 353.2 & 70.9 & 22 \\
\hline 260 & 516.0 & 25 & 508.3 & 98.5 & 25 & 498.2 & 96.6 & 25 & 475.7 & 92.2 & 25 & 364.5 & 70.6 & 22 \\
\hline 288 & 524.7 & 25 & 522.1 & 99.5 & 25 & 511.7 & 97.5 & 25 & 488.9 & 93.2 & 25 & 374.3 & 71.3 & 22 \\
\hline 316 & 532.7 & 25 & 527.0 & 98.9 & 25 & 520.1 & 97.6 & 25 & 496.6 & 93.2 & 25 & 376.0 & 70.6 & 22 \\
\hline 344 & 546.8 & 25 & 528.0 & 96.5 & 25 & 537.0 & 98.2 & 25 & 504.5 & 92.3 & 25 & 384.4 & 70.3 & 22 \\
\hline 372 & 555.4 & 25 & 547.9 & 98.7 & 25 & 545.8 & 98.3 & 25 & 510.5 & 91.9 & 25 & 389.2 & 70.1 & 22 \\
\hline 400 & 555.2 & 25 & 557.2 & 100.4 & 25 & 551.4 & 99.3 & 25 & 521.6 & 93.9 & 25 & 392.1 & 70.6 & 22 \\
\hline 428 & 566.3 & 25 & 563.6 & 99.5 & 25 & 564.7 & 99.7 & 25 & 528.6 & 93.3 & 25 & 400.4 & 70.7 & 22 \\
\hline 456 & 576.3 & 25 & 568.6 & 98.7 & 25 & 571.8 & 99.2 & 25 & 533.3 & 92.5 & 25 & 404.9 & 70.3 & 22 \\
\hline 484 & 577.8 & 25 & 575.4 & 99.6 & 25 & 579.0 & 100.2 & 25 & 534.8 & 92.6 & 25 & 406.8 & 70.4 & 22 \\
\hline 512 & 583.2 & 25 & 589.7 & 101.1 & 25 & 585.6 & 100.4 & 25 & 541.8 & 92.9 & 25 & 411.6 & 70.6 & 22 \\
\hline 540 & 584.6 & 25 & 587.1 & 100.4 & 25 & 588.6 & 100.7 & 25 & 546.7 & 93.5 & 25 & 415.1 & 71.0 & 22 \\
\hline 568 & 582.9 & 25 & 587.4 & 100.8 & 25 & 596.8 & 102.4 & 25 & 549.6 & 94.3 & 25 & 416.1 & 71.4 & 22 \\
\hline 596 & 583.2 & 24 & 598.1 & 102.5 & 24 & 600.8 & 103.0 & 25 & 552.7 & 94.8 & 25 & 417.4 & 71.6 & 22 \\
\hline 624 & 592.3 & 22 & 593.5 & 100.2 & 24 & 604.1 & 102.0 & 25 & 551.4 & 93.1 & 25 & 418.5 & 70.7 & 22 \\
\hline 652 & 585.7 & 22 & 587.4 & 100.3 & 24 & 610.4 & 104.2 & 25 & 565.4 & 96.5 & 24 & 420.5 & 71.8 & 21 \\
\hline 680 & 584.2 & 22 & 590.5 & 101.1 & 23 & 609.3 & 104.3 & 24 & 571.4 & 97.8 & 22 & 416.1 & 71.2 & 20 \\
\hline 708 & 588.5 & 20 & 586.9 & 99.7 & 23 & 606.7 & 103.1 & 23 & 556.5 & 94.6 & 22 & 403.0 & 68.5 & 20 \\
\hline EOS & 581.2 & 18 & 585.8 & 100.8 & 23 & 594.5 & 102.3 & 23 & 569.5 & 98.0 & 21 & 408.7 & 70.3 & 20 \\
\hline
\end{tabular}

EOS $=$ end of study; No. of litters $=$ number of litters represented in weight average.

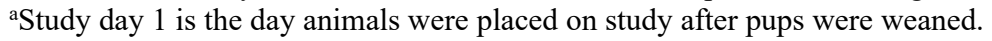

${ }^{\mathrm{b}}$ Average weights shown are means of litter means. 
Table 10. Summary of Survival and Mean Body Weights of Female Rats in the Perinatal and Postweaning Two-year Feed Study of Di(2-ethylhexyl) Phthalate

\begin{tabular}{|c|c|c|c|c|c|c|c|c|c|c|c|c|c|c|}
\hline \multirow[b]{2}{*}{$\begin{array}{c}\text { Study } \\
\text { Day }^{\mathrm{a}}\end{array}$} & \multicolumn{2}{|c|}{ 0 ppm } & \multicolumn{3}{|c|}{300 ppm } & \multicolumn{3}{|c|}{$1,000 \mathrm{ppm}$} & \multicolumn{3}{|c|}{$3,000 \mathrm{ppm}$} & \multicolumn{3}{|c|}{$10,000 \mathrm{ppm}$} \\
\hline & $\begin{array}{l}\text { Av. } \\
\text { Wt. } \\
(g)^{b}\end{array}$ & $\begin{array}{c}\text { No. of } \\
\text { Litters }\end{array}$ & $\begin{array}{l}\text { Av. } \\
\text { Wt. } \\
\text { (g) } \\
\end{array}$ & $\begin{array}{c}\text { Wt. } \\
\text { (\% of } \\
\text { Controls) } \\
\end{array}$ & $\begin{array}{l}\text { No. of } \\
\text { Litters }\end{array}$ & $\begin{array}{l}\text { Av. } \\
\text { Wt. } \\
\text { (g) }\end{array}$ & $\begin{array}{c}\text { Wt. } \\
\text { (\% of } \\
\text { Controls) } \\
\end{array}$ & $\begin{array}{c}\text { No. of } \\
\text { Litters }\end{array}$ & $\begin{array}{l}\text { Av. } \\
\text { Wt. } \\
\text { (g) }\end{array}$ & $\begin{array}{c}\text { Wt. } \\
\text { (\% of } \\
\text { Controls) } \\
\end{array}$ & $\begin{array}{l}\text { No. of } \\
\text { Litters }\end{array}$ & $\begin{array}{l}\text { Av. } \\
\text { Wt. } \\
\text { (g) }\end{array}$ & $\begin{array}{c}\text { Wt. } \\
\text { (\% of } \\
\text { Controls) } \\
\end{array}$ & $\begin{array}{l}\text { No. of } \\
\text { Litters }\end{array}$ \\
\hline 1 & 49.8 & 25 & 51.5 & 103.3 & 25 & 49.2 & 98.7 & 25 & 48.7 & 97.6 & 25 & 26.0 & 52.1 & 25 \\
\hline 8 & 77.5 & 25 & 78.6 & 101.4 & 25 & 75.6 & 97.5 & 25 & 74.3 & 95.9 & 25 & 36.9 & 47.6 & 22 \\
\hline 15 & 112.7 & 25 & 111.2 & 98.6 & 25 & 107.9 & 95.7 & 25 & 107.4 & 95.2 & 25 & 53.5 & 47.5 & 22 \\
\hline 22 & 141.8 & 25 & 141.7 & 99.9 & 25 & 138.0 & 97.3 & 25 & 137.8 & 97.2 & 25 & 73.1 & 51.5 & 22 \\
\hline 29 & 162.1 & 25 & 164.7 & 101.6 & 25 & 159.7 & 98.5 & 25 & 160.7 & 99.1 & 25 & 94.4 & 58.2 & 22 \\
\hline 36 & 181.3 & 25 & 187.3 & 103.3 & 25 & 180.5 & 99.6 & 25 & 180.7 & 99.7 & 25 & 114.5 & 63.1 & 22 \\
\hline 43 & 193.3 & 25 & 200.7 & 103.8 & 25 & 193.4 & 100.0 & 25 & 196.1 & 101.4 & 25 & 132.4 & 68.5 & 22 \\
\hline 50 & 206.7 & 25 & 211.4 & 102.3 & 25 & 205.2 & 99.3 & 25 & 206.5 & 99.9 & 25 & 147.9 & 71.5 & 22 \\
\hline 57 & 216.8 & 25 & 223.2 & 103.0 & 25 & 215.7 & 99.5 & 25 & 216.9 & 100.1 & 25 & 161.1 & 74.3 & 22 \\
\hline 64 & 224.0 & 25 & 232.0 & 103.6 & 25 & 223.7 & 99.9 & 25 & 224.1 & 100.0 & 25 & 171.9 & 76.8 & 22 \\
\hline 71 & 231.0 & 25 & 238.3 & 103.1 & 25 & 230.4 & 99.7 & 25 & 232.2 & 100.5 & 25 & 181.5 & 78.6 & 22 \\
\hline 78 & 236.4 & 25 & 242.1 & 102.4 & 25 & 233.7 & 98.9 & 25 & 235.8 & 99.8 & 25 & 188.0 & 79.6 & 22 \\
\hline 85 & 240.9 & 25 & 246.2 & 102.2 & 25 & 241.3 & 100.2 & 25 & 241.3 & 100.1 & 25 & 195.1 & 81.0 & 22 \\
\hline 92 & 245.6 & 25 & 249.0 & 101.4 & 25 & 245.8 & 100.1 & 25 & 245.4 & 99.9 & 25 & 199.2 & 81.1 & 22 \\
\hline 120 & 259.1 & 25 & 259.5 & 100.1 & 25 & 257.8 & 99.5 & 25 & 258.6 & 99.8 & 25 & 212.8 & 82.1 & 22 \\
\hline 148 & 268.6 & 25 & 273.3 & 101.8 & 25 & 266.2 & 99.1 & 25 & 269.0 & 100.2 & 25 & 222.0 & 82.7 & 22 \\
\hline 176 & 278.4 & 25 & 280.9 & 100.9 & 25 & 274.2 & 98.5 & 25 & 275.9 & 99.1 & 25 & 230.6 & 82.8 & 22 \\
\hline 204 & 283.3 & 25 & 282.1 & 99.6 & 25 & 282.1 & 99.6 & 25 & 282.3 & 99.6 & 25 & 232.8 & 82.2 & 22 \\
\hline 232 & 290.7 & 25 & 291.7 & 100.4 & 25 & 285.8 & 98.3 & 25 & 286.4 & 98.5 & 25 & 237.1 & 81.6 & 22 \\
\hline 260 & 297.0 & 25 & 300.4 & 101.1 & 25 & 290.8 & 97.9 & 25 & 293.7 & 98.9 & 25 & 240.6 & 81.0 & 22 \\
\hline 288 & 302.4 & 25 & 303.1 & 100.2 & 25 & 296.8 & 98.1 & 25 & 297.9 & 98.5 & 25 & 244.2 & 80.8 & 22 \\
\hline 316 & 301.0 & 25 & 305.3 & 101.4 & 25 & 300.9 & 100.0 & 25 & 302.3 & 100.4 & 25 & 244.9 & 81.4 & 22 \\
\hline 344 & 311.2 & 25 & 311.1 & 100.0 & 25 & 306.0 & 98.3 & 25 & 302.0 & 97.0 & 25 & 247.8 & 79.6 & 22 \\
\hline 372 & 314.7 & 25 & 319.2 & 101.4 & 25 & 307.2 & 97.6 & 25 & 304.4 & 96.7 & 25 & 247.5 & 78.7 & 22 \\
\hline 400 & 320.6 & 25 & 325.9 & 101.6 & 25 & 314.2 & 98.0 & 25 & 311.8 & 97.2 & 25 & 250.0 & 78.0 & 22 \\
\hline 428 & 325.4 & 25 & 330.2 & 101.5 & 25 & 320.9 & 98.6 & 25 & 310.6 & 95.5 & 25 & 250.4 & 76.9 & 22 \\
\hline 456 & 331.4 & 25 & 335.0 & 101.1 & 25 & 327.5 & 98.8 & 25 & 315.8 & 95.3 & 25 & 251.1 & 75.8 & 22 \\
\hline 484 & 339.0 & 25 & 345.3 & 101.9 & 25 & 332.7 & 98.2 & 25 & 326.2 & 96.2 & 25 & 253.4 & 74.8 & 22 \\
\hline 512 & 348.5 & 25 & 355.8 & 102.1 & 25 & 344.8 & 98.9 & 25 & 332.6 & 95.4 & 25 & 253.3 & 72.7 & 22 \\
\hline 540 & 350.3 & 24 & 344.4 & 98.3 & 24 & 348.1 & 99.4 & 25 & 330.2 & 94.3 & 25 & 256.7 & 73.3 & 22 \\
\hline 568 & 358.2 & 24 & 354.9 & 99.1 & 24 & 356.2 & 99.4 & 25 & 335.8 & 93.8 & 24 & 258.5 & 72.2 & 22 \\
\hline 596 & 361.9 & 24 & 353.6 & 97.7 & 23 & 357.9 & 98.9 & 24 & 342.5 & 94.6 & 24 & 257.0 & 71.0 & 22 \\
\hline 624 & 368.8 & 24 & 361.6 & 98.1 & 23 & 367.2 & 99.6 & 24 & 348.0 & 94.4 & 24 & 253.1 & 68.6 & 21 \\
\hline 652 & 378.9 & 24 & 368.8 & 97.3 & 23 & 376.1 & 99.3 & 23 & 351.4 & 92.7 & 24 & 251.4 & 66.3 & 20 \\
\hline 680 & 379.7 & 24 & 369.5 & 97.3 & 23 & 379.6 & 100.0 & 23 & 344.5 & 90.7 & 22 & 252.3 & 66.4 & 20 \\
\hline 708 & 376.4 & 23 & 359.5 & 95.5 & 21 & 380.1 & 101.0 & 23 & 338.2 & 89.9 & 21 & 252.6 & 67.1 & 19 \\
\hline EOS & 374.3 & 22 & 371.0 & 99.1 & 21 & 391.4 & 104.6 & 23 & 337.4 & 90.1 & 20 & 255.6 & 68.3 & 18 \\
\hline
\end{tabular}

EOS = end of study; No. of litters = number of litters represented in weight average.

aStudy day 1 is the day animals were placed on study after pups were weaned.

${ }^{\mathrm{b}}$ Average weights shown are means of litter means. 

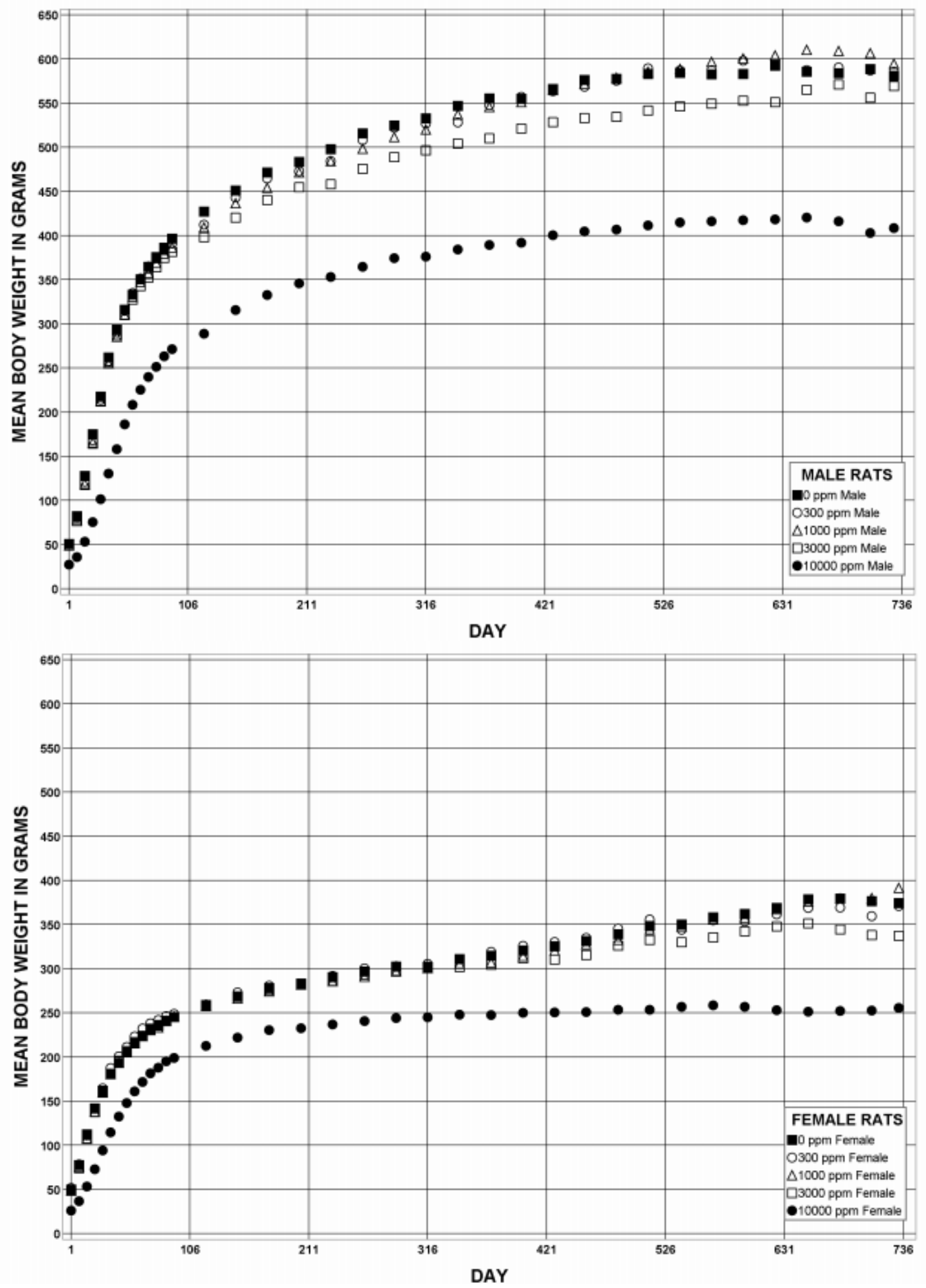

Figure 4. Growth Curves for Rats Exposed to Di(2-ethylhexyl) Phthalate in the Perinatal and Postweaning Two-year Feed Study 
Table 11. Summary of Feed and Di(2-ethylhexyl) Phthalate Consumption of Male Rats in the Perinatal and Postweaning Two-year Feed Study

\begin{tabular}{cccccccccc}
\hline \multirow{2}{*}{ Week } & $\mathbf{0 ~ p p m}$ & \multicolumn{2}{c}{$\mathbf{3 0 0} \mathbf{~ p p m}$} & \multicolumn{2}{c}{$\mathbf{1 , 0 0 0} \mathbf{~ p p m}$} & \multicolumn{2}{c}{$\mathbf{3 , 0 0 0} \mathbf{~ p p m}$} & \multicolumn{2}{c}{$\mathbf{1 0 , 0 0 0} \mathbf{~ p p m}$} \\
\cline { 2 - 9 } & $\begin{array}{c}\text { Feed } \\
(\mathbf{g} / \mathbf{d a y})^{\mathbf{a}}\end{array}$ & $\begin{array}{c}\text { Feed } \\
(\mathbf{g} / \mathbf{d a y})\end{array}$ & $\begin{array}{c}\text { Dose } \\
(\mathbf{m g} / \mathbf{k g} / \mathbf{d a y})^{\mathbf{b}}\end{array}$ & $\begin{array}{c}\text { Feed } \\
(\mathbf{g} / \mathbf{d a y})\end{array}$ & $\begin{array}{c}\text { Dose } \\
(\mathbf{m g} / \mathbf{k g} / \mathbf{d a y})\end{array}$ & $\begin{array}{c}\text { Feed } \\
(\mathbf{g} / \mathbf{d a y})\end{array}$ & $\begin{array}{c}\text { Dose } \\
(\mathbf{m g} / \mathbf{k g} / \mathbf{d a y})\end{array}$ & $\begin{array}{c}\text { Feed } \\
(\mathbf{g} / \mathbf{d a y})\end{array}$ & $\begin{array}{c}\text { Dose } \\
(\mathbf{m g} / \mathbf{k g} / \mathbf{d a y})\end{array}$ \\
\hline 1 & 9.7 & 9.8 & 58.2 & 9.5 & 193.2 & 8.8 & 540.0 & 2.5 & 908.5 \\
13 & 25.0 & 24.0 & 18.8 & 24.4 & 64.3 & 25.3 & 202.4 & 21.0 & 797.7 \\
54 & 27.7 & 28.6 & 15.7 & 26.6 & 48.7 & 28.6 & 168.0 & 22.3 & 569.5 \\
102 & 26.6 & 27.6 & 14.1 & 25.0 & 41.3 & 29.0 & 154.4 & 27.4 & 681.4 \\
\hline
\end{tabular}

${ }^{\mathrm{a}}$ Grams of feed consumed per animal per day.

${ }^{b}$ Milligrams of di(2-ethylhexyl) phthalate consumed/kilogram body weight/day.

Table 12. Summary of Feed and Di(2-ethylhexyl) Phthalate Consumption of Female Rats in the Perinatal and Postweaning Two-year Feed Study

\begin{tabular}{cccccccccc}
\hline \multirow{2}{*}{ Week } & $\mathbf{0 ~} \mathbf{~ p p m}$ & \multicolumn{2}{c}{$\mathbf{3 0 0} \mathbf{~ p p m}$} & \multicolumn{2}{c}{$\mathbf{1 , 0 0 0} \mathbf{~ p p m}$} & \multicolumn{2}{c}{$\mathbf{3 , 0 0 0} \mathbf{~ p p m}$} & \multicolumn{2}{c}{$\mathbf{1 0 , 0 0 0} \mathbf{~ p p m}$} \\
\cline { 2 - 9 } & $\begin{array}{c}\text { Feed } \\
(\mathbf{g} / \mathbf{d a y})^{\mathbf{a}}\end{array}$ & $\begin{array}{c}\text { Feed } \\
(\mathbf{g} / \mathbf{d a y})\end{array}$ & $\begin{array}{c}\text { Dose } \\
(\mathbf{m g} / \mathbf{k g} / \mathbf{d a y})^{\mathbf{b}}\end{array}$ & $\begin{array}{c}\text { Feed } \\
(\mathbf{g} / \mathbf{d a y})\end{array}$ & $\begin{array}{c}\text { Dose } \\
(\mathbf{m g} / \mathbf{k g} / \mathbf{d a y})\end{array}$ & $\begin{array}{c}\text { Feed } \\
(\mathbf{g} / \mathbf{d a y})\end{array}$ & $\begin{array}{c}\text { Dose } \\
(\mathbf{m g} / \mathbf{k g} / \mathbf{d a y})\end{array}$ & $\begin{array}{c}\text { Feed } \\
(\mathbf{g} / \mathbf{d a y})\end{array}$ & $\begin{array}{c}\text { Dose } \\
(\mathbf{m g} / \mathbf{k g} / \mathbf{d a y})\end{array}$ \\
\hline 1 & 9.1 & 9.2 & 53.6 & 9.0 & 182.9 & 8.8 & 542.5 & 2.7 & $1,076.8$ \\
13 & 15.8 & 16.0 & 19.5 & 16.5 & 68.4 & 16.8 & 208.9 & 15.5 & 796.9 \\
54 & 16.9 & 16.6 & 15.6 & 16.5 & 53.6 & 16.9 & 166.6 & 17.6 & 709.8 \\
102 & 20.3 & 20.3 & 17.0 & 21.0 & 56.1 & 23.2 & 204.4 & 22.9 & 899.7 \\
\hline
\end{tabular}

${ }^{\mathrm{a}}$ Grams of feed consumed per animal per day.

${ }^{b}$ Milligrams of di(2-ethylhexyl) phthalate consumed per kilogram body weight per day.

\section{Pathology}

This section describes statistically significant or biologically noteworthy changes in the incidences of gross lesions, neoplasms, and/or nonneoplastic lesions of the liver, pancreas, male and female reproductive organs, kidney, heart, bone marrow, and pituitary gland. Summaries of the incidences of neoplasms and nonneoplastic lesions, individual animal neoplasm diagnoses, and statistical analyses of primary neoplasms that occurred with an incidence of at least $5 \%$ in at least one animal group are presented as supplemental data in Appendix H.

Liver: There were significant increases in the incidences of hepatocellular adenoma $(10,000 \mathrm{ppm}$ males and 3,000 ppm females) and carcinoma (10,000 ppm females) relative to control animals and a positive trend in incidence with increasing exposure concentration in males for hepatocellular carcinomas (Table 13). The incidences of hepatocellular adenoma or carcinoma (combined) were significantly increased in the $10,000 \mathrm{ppm}$ male and female groups and in the 3,000 ppm female group relative to control animals. Hepatocellular adenomas were characterized by regions that were sharply demarcated from surrounding liver parenchyma, nodular, and compressing adjacent normal hepatocytes, with loss of normal lobular architecture and an irregular growth pattern. The liver plates typically impinged obliquely on the surrounding liver parenchyma. The hepatocytes within an adenoma generally varied in size. Hepatocellular carcinomas were characterized by one or more of the following features: local infiltrating growth and/or distinct lack of demarcation with the adjacent tissue, the presence of trabeculae composed 
of multiple layers of hepatocytes, cellular pleomorphism, loss of normal lobular architecture, regions of hemorrhage and/or necrosis, and increased mitotic figures.

There were significant increases in the incidences of many nonneoplastic liver lesions in DEHPexposed groups relative to the control groups (Table 13). The incidences of hepatocellular cytoplasmic alteration were significantly increased in 3,000 and 10,000 ppm males and in all exposed groups of females relative to control animals. There were significant increases in the incidence of hepatocellular hypertrophy in the $10,000 \mathrm{ppm}$ males and in the 1,000,3,000, and $10,000 \mathrm{ppm}$ females. Significant increases in the incidence of pigment were observed in the $1,000,3,000$, and 10,000 ppm males and in all exposed groups of females. There were significant increases in the incidence of hepatocellular necrosis in the $10,000 \mathrm{ppm}$ males relative to control animals. The incidence of hepatocellular eosinophilic foci was significantly increased in $10,000 \mathrm{ppm}$ females over that of the control group, and a positive trend was seen in males with increasing exposure concentration. The incidence of hepatocellular basophilic foci was significantly increased in the $10,000 \mathrm{ppm}$ males relative to the control group, and a positive trend with increasing exposure occurred in females. There was a significant increase in the incidence of bile duct hyperplasia in 3,000 ppm females compared to control animals.

Hepatocellular cytoplasmic alteration was characterized by hepatocytes that were expanded with eosinophilic granular cytoplasm (Figure 5). A four-grade severity scale reflected the degree of tissue affected in the section of liver that was evaluated histologically: minimal (grade 1), up to $25 \%$ of hepatocyte involvement; mild (grade 2 ), $26 \%$ to $50 \%$ of hepatocyte involvement; moderate (grade 3 ), $51 \%$ to $75 \%$ of hepatocyte involvement; and marked (grade 4), at least $76 \%$ of hepatocyte involvement. Hepatocellular hypertrophy often occurred in conjunction with cytoplasmic alteration and/or pigment. Hypertrophy was characterized by enlargement of the hepatocytes. In lesser affected animals, hypertrophy was confined to centrilobular regions, but in more severely affected animals, hypertrophy extended into the midzonal and periportal areas. A four-grade severity scale was used: minimal (grade 1), up to $10 \%$ of hepatocyte involvement; mild (grade 2), $11 \%$ to $25 \%$ of hepatocyte involvement; moderate (grade 3 ), up to $50 \%$ of hepatocyte involvement; and severe (grade 4 ), $>51 \%$ of hepatocyte involvement. Hypertrophy was generally centrilobular and often involved only a few cells per lobule. Although hypertrophy was only occasionally observed in males (at the 3,000 and 10,000 ppm exposure concentrations), in females it exhibited a concentration-responsive significant increase in incidence (but not severity) starting at the $1,000 \mathrm{ppm}$ exposure concentration.

Pigment only occurred in exposed animals and was characterized by a pale gold-colored pigment within the hepatocellular cytoplasm (Figure 5). A four-grade severity scale was used: minimal (grade 1), up to $30 \%$ of hepatocytes contained pigment; mild (grade 2), $31 \%$ to $50 \%$ of hepatocytes contained pigment; moderate (grade 3 ), $>51 \%$ of hepatocytes contained pigment; and marked (grade 4 ), $>51 \%$ of hepatocytes contained pigment and the pigment was very dense. Hepatocellular necrosis was characterized by multiple adjacent hepatocytes that were swollen with increased eosinophilia, karyorrhectic nuclear debris, with or without accompanying inflammatory cells. A four-grade severity scale was used: minimal (grade 1), up to three focal areas of necrosis present; mild (grade 2), necrosis in more than three involved regions or up to $25 \%$ of the liver; moderate (grade 3 ), necrosis in $26 \%$ to $60 \%$ of the liver; and severe (grade 4 ), necrosis in $>61 \%$ of the liver. 
Eosinophilic and basophilic hepatocellular foci were diagnosed when there was an enlargement of the hepatocytes with increased acidophilic or basophilic staining, respectively, compared with the surrounding normal liver cells. Foci typically had a discrete lesion margin, where attenuated hepatocytes at the lesion margin (compression) involved $<70 \%$ of the lesion circumference. There was preservation of lobular architecture and absence of cellular atypia. The distinction between large foci (usually eosinophilic or mixed) and hepatocellular adenomas was made on the basis of retention of normal lobular architecture in the foci, greater size of hepatocellular adenomas (usually measuring at least $3 \mathrm{~mm}$ ), and presence of compression or bulging from the liver surface along $>70 \%$ of the lesion circumference.

Bile duct hyperplasia was diagnosed when increased numbers of small bile ducts arose in portal regions. The biliary epithelium lining the ducts was well differentiated, forming normal ducts.

Table 13. Incidences of Neoplastic and Nonneoplastic Lesions of the Liver in Male and Female Rats in the Perinatal and Postweaning Two-year Feed Study of Di(2-ethylhexyl) Phthalate

\begin{tabular}{|c|c|c|c|c|c|}
\hline & O ppm & 300 ppm & $1,000 \mathrm{ppm}$ & $3,000 \mathrm{ppm}$ & $10,000 \mathrm{ppm}$ \\
\hline \multicolumn{6}{|l|}{ Male } \\
\hline $\mathbf{n}^{\mathbf{a}}$ & 50 & 49 & 50 & 50 & 49 \\
\hline Hepatocyte, Cytoplasmic Alteration ${ }^{b}$ & $0 * *$ & 0 & $1(1.0)^{\mathrm{c}}$ & $28 * *(1.3)$ & $37 * *(2.6)$ \\
\hline Hepatocyte, Hypertrophy & $0 * *$ & 0 & 0 & $3(1.3)$ & $17 * *(1.6)$ \\
\hline Pigment & $0 * *$ & $1(1.0)$ & $5 *(1.2)$ & $40 * *(1.7)$ & $38 * *(2.5)$ \\
\hline Necrosis & $3 * *(1.3)$ & $4(2.0)$ & $1(1.0)$ & $6(1.8)$ & $13 *(1.3)$ \\
\hline Eosinophilic Focus & $4 * *$ & 1 & 7 & 2 & 11 \\
\hline Basophilic Focus & $1 * *$ & 1 & 4 & 4 & $17 * *$ \\
\hline Bile Duct Hyperplasia & $13(1.2)$ & $18(1.1)$ & $13(1.2)$ & $21(1.0)$ & $15(1.1)$ \\
\hline \multicolumn{6}{|l|}{ Hepatocellular Adenoma $^{\mathrm{d}}$} \\
\hline Overall rate ${ }^{\mathrm{e}}$ & $0 / 50(0 \%)$ & $1 / 49(2 \%)$ & $0 / 50(0 \%)$ & $3 / 50(6 \%)$ & $8 / 49(16 \%)$ \\
\hline Rate per litters ${ }^{\mathrm{f}}$ & $0 / 25(0 \%)$ & $1 / 25(4 \%)$ & $0 / 25(0 \%)$ & $3 / 25(12 \%)$ & $7 / 25(28 \%)$ \\
\hline Adjusted rate ${ }^{g}$ & $0 \%$ & $2.4 \%$ & $0 \%$ & $6.7 \%$ & $22.3 \%$ \\
\hline Rao-Scott-adjusted Poly-3 test ${ }^{\mathrm{h}}$ & $\mathrm{p}<0.001$ & $\mathrm{p}=0.604$ & (e) & $\mathrm{p}=0.246$ & $\mathrm{p}=0.018$ \\
\hline \multicolumn{6}{|l|}{ Hepatocellular Carcinoma $^{\mathrm{i}}$} \\
\hline Overall rate & $1 / 50(2 \%)$ & $0 / 49(0 \%)$ & $0 / 50(0 \%)$ & $0 / 50(0 \%)$ & $3 / 49(6 \%)$ \\
\hline Rate per litters & $1 / 25(4 \%)$ & $0 / 25(0 \%)$ & $0 / 25(0 \%)$ & $0 / 25(0 \%)$ & $3 / 25(12 \%)$ \\
\hline Adjusted rate & $2.6 \%$ & $0 \%$ & $0 \%$ & $0 \%$ & $8.7 \%$ \\
\hline Rao-Scott-adjusted Poly-3 test & $\mathrm{p}=0.038$ & $\mathrm{p}=0.589 \mathrm{~N}$ & $\mathrm{p}=0.587 \mathrm{~N}$ & $\mathrm{p}=0.587 \mathrm{~N}$ & $\mathrm{p}=0.341$ \\
\hline \multicolumn{6}{|c|}{ Hepatocellular Adenoma or Carcinoma (Combined) ${ }^{\mathrm{j}}$} \\
\hline Overall rate & $1 / 50(2 \%)$ & $1 / 49(2 \%)$ & $0 / 50(0 \%)$ & $3 / 50(6 \%)$ & $11 / 49(22 \%)$ \\
\hline Rate per litters & $1 / 25(4 \%)$ & $1 / 25(4 \%)$ & $0 / 25(0 \%)$ & $3 / 25(12 \%)$ & $9 / 25(36 \%)$ \\
\hline Adjusted rate & $2.6 \%$ & $2.4 \%$ & $0 \%$ & $6.7 \%$ & $30.6 \%$ \\
\hline Rao-Scott-adjusted Poly-3 test & $\mathrm{p}<0.001$ & $\mathrm{p}=0.750 \mathrm{~N}$ & $\mathrm{p}=0.565 \mathrm{~N}$ & $\mathrm{p}=0.429$ & $\mathrm{p}=0.009$ \\
\hline
\end{tabular}




\begin{tabular}{|c|c|c|c|c|c|}
\hline & 0 ppm & 300 ppm & 1,000 ppm & 3,000 ppm & $10,000 \mathrm{ppm}$ \\
\hline \multicolumn{6}{|l|}{ Female } \\
\hline $\mathbf{n}$ & 49 & 50 & 50 & 50 & 48 \\
\hline Hepatocyte, Cytoplasmic Alteration & $0 * *$ & $4 *(1.0)$ & $7 * *(1.1)$ & $39 * *(1.6)$ & $39 * *(3.4)$ \\
\hline Hepatocyte, Hypertrophy & $0 * *$ & $2(2.0)$ & $5 *(1.6)$ & $9 * *(1.6)$ & $34 * *(2.4)$ \\
\hline Pigment & $0 * *$ & $6^{*}(1.0)$ & $14 * *(1.1)$ & $36 * *(1.6)$ & $40 * *(2.6)$ \\
\hline Necrosis & $3(2.0)$ & $9(2.3)$ & $3(2.3)$ & $7(1.9)$ & $8(2.3)$ \\
\hline Eosinophilic Focus & $3 * *$ & 4 & 4 & 7 & $12 * *$ \\
\hline Basophilic Focus & $4 * *$ & 5 & 3 & 2 & 10 \\
\hline Bile Duct Hyperplasia & $9(1.2)$ & $13(1.0)$ & $13(1.2)$ & $21 *(1.1)$ & $8(1.1)$ \\
\hline \multicolumn{6}{|l|}{ Hepatocellular Adenoma ${ }^{\mathrm{k}}$} \\
\hline Overall rate & $1 / 49(2 \%)$ & $0 / 50(0 \%)$ & $5 / 50(10 \%)$ & $9 / 50(18 \%)$ & $5 / 48(10 \%)$ \\
\hline Rate per litters & $1 / 25(4 \%)$ & $0 / 25(0 \%)$ & $4 / 25(6 \%)$ & $7 / 25(28 \%)$ & $5 / 25(20 \%)$ \\
\hline Adjusted rate & $2.4 \%$ & $0 \%$ & $11.8 \%$ & $20.9 \%$ & $13.8 \%$ \\
\hline Rao-Scott-adjusted Poly-3 test & $\mathrm{p}=0.089$ & $\mathrm{p}=0.587 \mathrm{~N}$ & $\mathrm{p}=0.170$ & $\mathrm{p}=0.033$ & $\mathrm{p}=0.126$ \\
\hline \multicolumn{6}{|l|}{ Hepatocellular Carcinoma ${ }^{1}$} \\
\hline Overall rate & $0 / 49(0 \%)$ & $0 / 50(0 \%)$ & $0 / 50(0 \%)$ & $0 / 50(0 \%)$ & $8 / 48(17 \%)$ \\
\hline Rate per litters & $0 / 25(0 \%)$ & $0 / 25(0 \%)$ & $0 / 25(0 \%)$ & $0 / 25(0 \%)$ & $7 / 25(28 \%)$ \\
\hline Adjusted rate & $0 \%$ & $0 \%$ & $0 \%$ & $0 \%$ & $21.8 \%$ \\
\hline Rao-Scott-adjusted Poly-3 test & $\mathrm{p}<0.001$ & (e) & (e) & (e) & $\mathrm{p}=0.023$ \\
\hline \multicolumn{6}{|c|}{ Hepatocellular Adenoma or Carcinoma (Combined) ${ }^{\mathrm{m}}$} \\
\hline Overall rate & $1 / 49(2 \%)$ & $0 / 50(0 \%)$ & $5 / 50(10 \%)$ & $9 / 50(18 \%)$ & $13 / 48(27 \%)$ \\
\hline Rate per litters & $1 / 25(4 \%)$ & $0 / 25(0 \%)$ & $4 / 25(16 \%)$ & $7 / 25(28 \%)$ & $11 / 25(44 \%)$ \\
\hline Adjusted rate & $2.4 \%$ & $0 \%$ & $11.8 \%$ & $20.9 \%$ & $35.4 \%$ \\
\hline Rao-Scott-adjusted Poly- 3 test & $\mathrm{p}<0.001$ & $\mathrm{p}=0.568 \mathrm{~N}$ & $\mathrm{p}=0.158$ & $\mathrm{p}=0.028$ & $\mathrm{p}=0.002$ \\
\hline
\end{tabular}

Statistical significance for an exposure group indicates a significant pairwise test compared to the vehicle control group.

Statistical significance for the vehicle control group indicates a significant trend test.

*Significantly different $(\mathrm{p} \leq 0.05)$ from the control group by the Rao-Scott-adjusted Poly- 3 test; ** $\mathrm{p} \leq 0.01$.

(e) = value of statistic could not be computed.

${ }^{a}$ Number of animals with tissue examined microscopically.

${ }^{b}$ Number of animals with lesion.

${ }^{\mathrm{c}}$ Average severity grade of lesions in affected animals: $1=$ minimal, $2=$ mild, $3=$ moderate, $4=$ marked .

${ }^{\mathrm{d}}$ Historical control incidence for all routes of 2-year studies (mean \pm standard deviation): $2 / 489(0.44 \% \pm 0.88 \%)$;

range: $0 \%$ to $2 \%$.

${ }^{\mathrm{e}}$ Number of animals with neoplasm per number of animals necropsied.

fNumber of litters with neoplasm-bearing animals per number of litters examined at site.

gPoly-3 estimated neoplasm incidence after adjustment for intercurrent mortality.

heneath the control incidence is the $\mathrm{p}$ value associated with the trend test. Beneath the exposed group incidence are the $\mathrm{p}$ values corresponding to pairwise comparisons between the control group and that exposed group. The Rao-Scott test adjusts the Poly-3 test (which accounts for differential mortality in animals that do not reach study termination) for within-litter correlation. A

negative trend or a lower incidence in an exposure group is indicated by $\mathrm{N}$.

${ }^{i}$ Historical control incidence: $2 / 489(0.45 \% \pm 0.89 \%)$; range: $0 \%$ to $2 \%$.

${ }^{\mathrm{j}}$ Historical control incidence: $4 / 489(0.89 \% \pm 1.06 \%)$; range: $0 \%$ to $2 \%$.

${ }^{k}$ Historical control incidence: $15 / 489(2.65 \% \pm 2.59 \%)$; range: $0 \%$ to $8 \%$.

${ }^{1}$ Historical control incidence: $1 / 489(0.22 \% \pm 0.67 \%)$; range: $0 \%$ to $2 \%$.

${ }^{\mathrm{m}}$ Historical control incidence: $16 / 489(2.87 \% \pm 2.8 \%)$; range: $0 \%$ to $8 \%$. 
A)

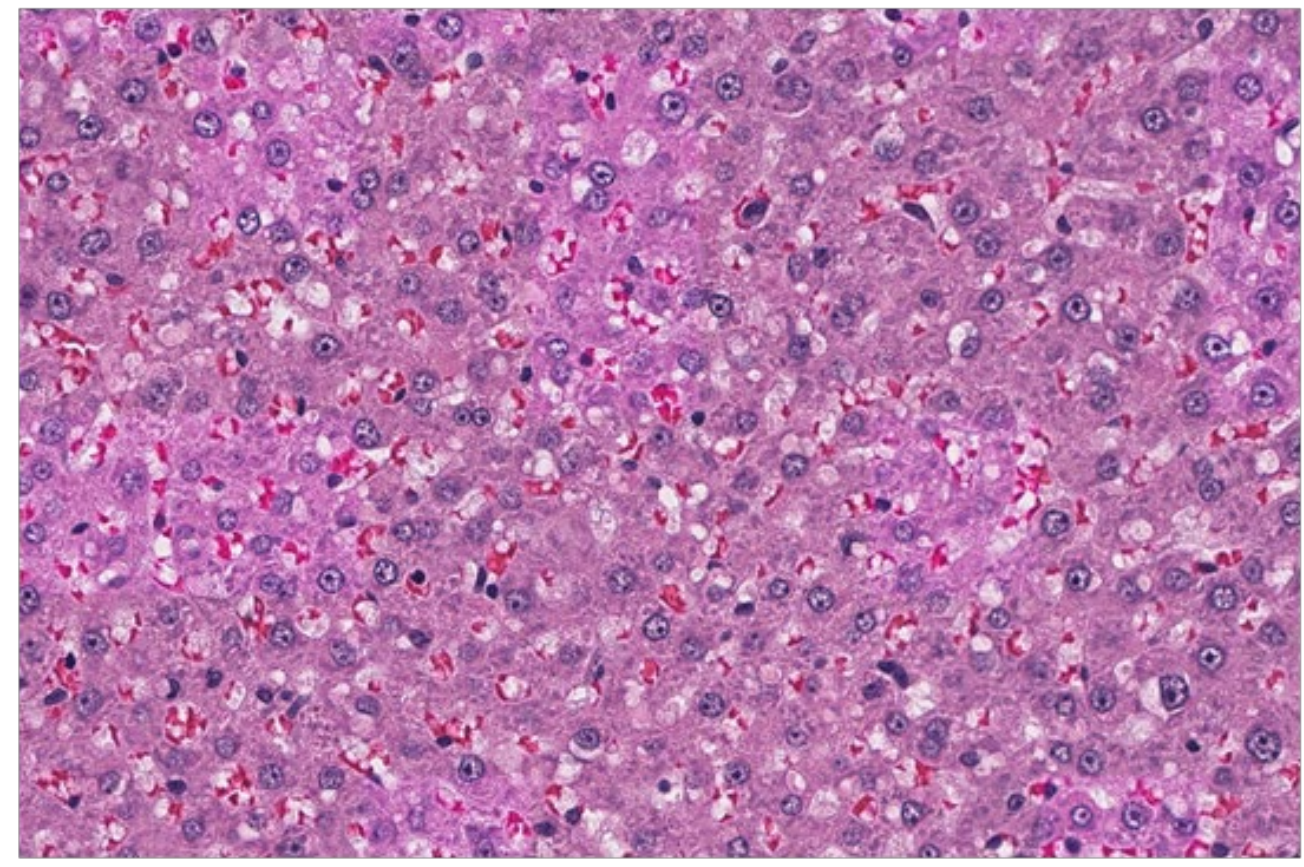

B)

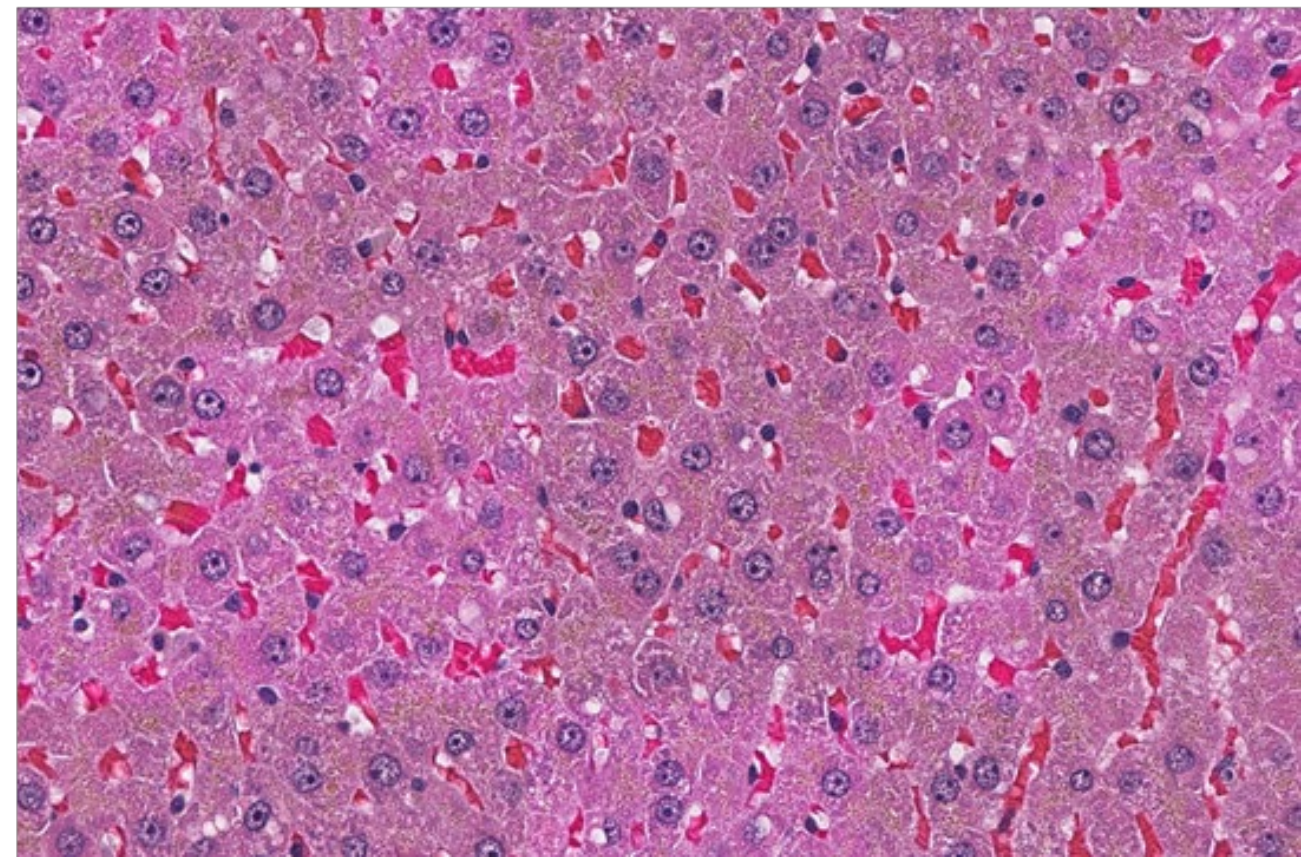

Figure 5. Hepatocellular Cytoplasmic Alteration with Pigment in Male Rats Exposed to Di(2-ethylhexyl) Phthalate in the Perinatal and Postweaning Two-year Feed Study (H\&E)

Compare normal liver from a male control rat (panel A) to liver from a male rat exposed to 10,000 ppm DEHP (panel B). Hepatocytes are expanded with eosinophilic granular cytoplasm and pigment at $40 \times$ magnification. 
Pancreas: In male rats, there were significant increases in the incidences of pancreatic acinar adenoma and pancreatic acinar adenoma or carcinoma (combined) in the 3,000 and 10,000 ppm groups compared to the control group (Table 14). In males, acinar carcinomas were present in the 3,000 and 10,000 ppm groups, but the incidences were not significant (Table 14). In females, acinar adenomas were observed in the 3,000 and 10,000 ppm groups, but the incidences were not significant (Table 14). Pancreatic acinar adenomas were characterized as distinct nodular masses that were not contiguous with the adjacent parenchyma, which were $>3 \mathrm{~mm}$ in diameter, and that compressed the adjacent tissue; pleomorphism or atypia was rarely present. Pancreatic acinar carcinomas were typically larger than adenomas and frequently exhibited cellular pleomorphism and atypia; invasion or metastasis was pathognomonic. Scirrhous reactions were occasionally present, characterized by dense fibrous or connective tissue. Pancreatic acinar hyperplasias were higher in the 1,000,3000, and 10,000 ppm male groups and in the 3,000 and 10,000 ppm female groups compared to the control group, but the differences were not significant.

There was a clear morphological continuum from focal acinar hyperplasia to adenoma and to carcinoma. Pancreatic acinar hyperplasia was characterized by circumscribed areas of enlarged acini that were $<3 \mathrm{~mm}$ in diameter and that were contiguous with the adjacent parenchyma. Severity grades were assigned using a four-grade scale: minimal (grade 1), no more than one lobule was affected and the lesion was smaller than $1 \mathrm{~mm}$; mild (grade 2), lesion was 1 to $2 \mathrm{~mm}$; moderate (grade 3), lesion was 2 to $3 \mathrm{~mm}$; and marked (grade 4), lesion was $3 \mathrm{~mm}$ but lacked features of an adenoma, such as compression. Severity grades were increased if multiple lesions were present.

Table 14. Incidences of Neoplastic and Nonneoplastic Lesions of the Pancreas in Male and Female Rats in the Perinatal and Postweaning Two-year Feed Study of Di(2-ethylhexyl) Phthalate

\begin{tabular}{|c|c|c|c|c|c|}
\hline & 0 ppm & 300 ppm & 1,000 ppm & 3,000 ppm & 10,000 ppm \\
\hline \multicolumn{6}{|l|}{ Male } \\
\hline $\mathbf{n}^{\mathbf{a}}$ & 50 & 49 & 50 & 50 & 49 \\
\hline Acinus, Hyperplasiab & $13(3.1)^{\mathrm{c}}$ & $9(2.6)$ & $16(3.3)$ & $25(3.5)$ & $15(3.3)$ \\
\hline \multicolumn{6}{|l|}{ Acinar Adenoma $^{\mathrm{d}}$} \\
\hline Overall rate $\mathrm{e}^{\mathrm{e}}$ & $10 / 50(20 \%)$ & $7 / 49(14 \%)$ & $8 / 50(16 \%)$ & $36 / 50(72 \%)$ & $22 / 49(45 \%)$ \\
\hline Rate per litters ${ }^{\mathrm{f}}$ & $8 / 25(32 \%)$ & $5 / 25(20 \%)$ & $8 / 25(32 \%)$ & $24 / 25(96 \%)$ & $18 / 25(72 \%)$ \\
\hline Adjusted rate & $26 \%$ & $16.6 \%$ & $16.9 \%$ & $77.9 \%$ & $62.5 \%$ \\
\hline Rao-Scott-adjusted Poly-3 test ${ }^{\mathrm{h}}$ & $\mathrm{p}<0.001$ & $\mathrm{p}=0.209 \mathrm{~N}$ & $\mathrm{p}=0.210 \mathrm{~N}$ & $\mathrm{p}<0.001$ & $\mathrm{p}<0.001$ \\
\hline \multicolumn{6}{|l|}{ Acinar Carcinoma ${ }^{\mathrm{i}}$} \\
\hline Overall rate & $0 / 50(0 \%)$ & $0 / 49(0 \%)$ & $0 / 50(0 \%)$ & $3 / 50(6 \%)$ & $1 / 49(2 \%)$ \\
\hline Rate per litters & $0 / 25(0 \%)$ & $0 / 25(0 \%)$ & $0 / 25(0 \%)$ & $3 / 25(12 \%)$ & $1 / 25(4 \%)$ \\
\hline Adjusted rate & $0 \%$ & $0 \%$ & $0 \%$ & $6.6 \%$ & $2.9 \%$ \\
\hline Rao-Scott-adjusted Poly-3 test & $\mathrm{p}=0.290$ & (e) & (e) & $\mathrm{p}=0.250$ & $\mathrm{p}=0.534$ \\
\hline \multicolumn{6}{|c|}{ Acinar Adenoma or Carcinoma (Combined $)^{\mathrm{j}}$} \\
\hline Overall rate & $10 / 50(20 \%)$ & $7 / 49(14 \%)$ & $8 / 50(16 \%)$ & $38 / 50(76 \%)$ & $22 / 49(45 \%)$ \\
\hline Rate per litters & $8 / 25(32 \%)$ & $5 / 25(20 \%)$ & $8 / 25(32 \%)$ & $25 / 25(100 \%)$ & $18 / 25(72 \%)$ \\
\hline
\end{tabular}




\begin{tabular}{lccccc}
\hline & $\mathbf{0 ~} \mathbf{~ p m}$ & $\mathbf{3 0 0} \mathbf{~ p p m}$ & $\mathbf{1 , 0 0 0} \mathbf{~ p p m}$ & $\mathbf{3 , 0 0 0} \mathbf{~ p p m}$ & $\mathbf{1 0 , 0 0 0} \mathbf{~ p p m}$ \\
\hline Adjusted rate & $26 \%$ & $16.6 \%$ & $16.9 \%$ & $81.2 \%$ & $62.5 \%$ \\
Rao-Scott-adjusted Poly-3 test & $\mathrm{p}<0.001$ & $\mathrm{p}=0.209 \mathrm{~N}$ & $\mathrm{p}=0.210 \mathrm{~N}$ & $\mathrm{p}<0.001$ & $\mathrm{p}<0.001$ \\
\hline
\end{tabular}

Female

\begin{tabular}{|c|c|c|c|c|c|}
\hline $\mathbf{n}$ & 49 & 50 & 50 & 50 & 48 \\
\hline Acinus, Hyperplasia & 0 & 0 & 0 & $2(1.5)$ & $3(2.0)$ \\
\hline \multicolumn{6}{|l|}{ Acinar Adenoma ${ }^{\mathrm{k}}$} \\
\hline Overall rate & $0 / 49(0 \%)$ & $0 / 50(0 \%)$ & $0 / 50(0 \%)$ & $2 / 50(4 \%)$ & $1 / 48(2 \%)$ \\
\hline Rate per litters & $0 / 25(0 \%)$ & $0 / 25(0 \%)$ & $0 / 25(0 \%)$ & $2 / 25(8 \%)$ & $1 / 25(4 \%)$ \\
\hline Adjusted rate & $0 \%$ & $0 \%$ & $0 \%$ & $4.6 \%$ & $2.8 \%$ \\
\hline Rao-Scott-adjusted Poly- 3 test & $\mathrm{p}=0.307$ & (e) & (e) & $p=0.366$ & $\mathrm{p}=0.561$ \\
\hline \multicolumn{6}{|l|}{ Acinar Carcinoma $^{1}$} \\
\hline Overall rate & $0 / 49(0 \%)$ & $0 / 50(0 \%)$ & $0 / 50(0 \%)$ & $0 / 50(0 \%)$ & $0 / 48(0 \%)$ \\
\hline Rate per litters & $0 / 25(0 \%)$ & $0 / 25(0 \%)$ & $0 / 25(0 \%)$ & $0 / 25(0 \%)$ & $0 / 25(0 \%)$ \\
\hline Adjusted rate & $0 \%$ & $0 \%$ & $0 \%$ & $0 \%$ & $0 \%$ \\
\hline Rao-Scott-adjusted Poly-3 test & $-^{\mathrm{m}}$ & - & - & - & - \\
\hline \multicolumn{6}{|c|}{ Acinar Adenoma or Carcinoma $(\text { Combined })^{\mathrm{n}}$} \\
\hline Overall rate & $0 / 49(0 \%)$ & $0 / 50(0 \%)$ & $0 / 50(0 \%)$ & $2 / 50(4 \%)$ & $1 / 48(2 \%)$ \\
\hline Rate per litters & $0 / 25(0 \%)$ & $0 / 25(0 \%)$ & $0 / 25(0 \%)$ & $2 / 25(8 \%)$ & $1 / 25(4 \%)$ \\
\hline Adjusted rate & $0 \%$ & $0 \%$ & $0 \%$ & $4.6 \%$ & $2.8 \%$ \\
\hline Rao-Scott-adjusted Poly-3 test & $\mathrm{p}=0.307$ & (e) & (e) & $\mathrm{p}=0.366$ & $\mathrm{p}=0.561$ \\
\hline
\end{tabular}

(e) = value of statistic could not be computed.

${ }^{a}$ Number of animals with tissue examined microscopically.

${ }^{b}$ Number of animals with lesion.

${ }^{\mathrm{c}}$ Average severity grade of lesions in affected animals: $1=$ minimal, $2=$ mild, $3=$ moderate, $4=$ marked.

${ }^{\mathrm{d} H i s t o r i c a l}$ control incidence for all routes of 2-year studies (mean \pm standard deviation): $60 / 488(11.58 \% \pm 9.25 \%)$;

range: $0 \%$ to $28 \%$.

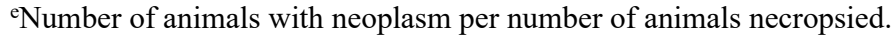

fNumber of litters with neoplasm-bearing animals per number of litters examined at site.

gPoly-3 estimated neoplasm incidence after adjustment for intercurrent mortality.

${ }^{\text {h} B e n e a t h}$ the control incidence is the $\mathrm{p}$ value associated with the trend test. Beneath the exposed group incidence are the $\mathrm{p}$ values corresponding to pairwise comparisons between the control group and that exposed group. The Rao-Scott test adjusts the Poly-3 test (which accounts for differential mortality in animals that do not reach study termination) for within-litter correlation. A negative trend or a lower incidence in an exposure group is indicated by $\mathrm{N}$.

iHistorical control incidence: $4 / 488(0.8 \% \pm 1.42 \%)$; range: $0 \%$ to $4 \%$.

${ }^{\mathrm{j} H}$ Historical control incidence: $62 / 488(12.03 \% \pm 9.16 \%)$; range: $0 \%$ to $28 \%$.

${ }^{k}$ Historical control incidence: $0 / 489$.

${ }^{1}$ Historical control incidence: $0 / 489$.

${ }^{m}$ Not applicable; no neoplasms in group.

${ }^{n}$ Historical control incidence: 0/489.

Gross lesions of the reproductive tract: Significantly increased incidences of several genitourinary abnormalities consistent with perturbation of developmental testosterone signaling and Wolffian duct differentiation were evident among 10,000 ppm DEHP-exposed males relative to control animals (Table 15). Small reproductive tissues were noted in 10,000 ppm males, including, but not limited to, the phallus, testes, epididymides, seminal vesicles, levator 
ani/bulbocavernosus (LABC) muscle, Cowper's glands, and prostate (Table 15; Appendix H). Findings in the testes (small) were particularly prevalent, occurring in all 10,000 ppm males that survived past the initial 2 weeks of the 2 -year study period relative to only two incidences in examined control rats (Table 15). Undescended testes (unilateral or bilateral) were noted in the abdomen in 19 (approximately 39\%) rats and the inguinal region in 4 (approximately 8\%) rats in the $10,000 \mathrm{ppm}$ group relative to a single incidence of undescended testes in the abdomen (bilateral) in control males. Findings commonly associated with testicular retention in the abdomen, such as the presence of a cranial suspensory ligament (approximately 10\%) and extended ( $>20 \mathrm{~mm}$; approximately $16 \%$ ) or absent gubernaculum (unilateral or bilateral; approximately $35 \%$ ), were also significantly increased in the $10,000 \mathrm{ppm}$ males relative to control animals (Table 15). Exposure-related increases in the left and right mean gubernaculum lengths were evident in 10,000 ppm males. Using a nonparametric (rank-based) analysis, a significant increase in the length of the right gubernaculum was observed in 10,000 ppm males. This corresponded to data in which the effect was largely associated with increased right gubernaculum lengths ( $\geq 35 \mathrm{~mm}$ ) in seven males in the $10,000 \mathrm{ppm}$ group compared to five animals with these lengths in the left gubernaculum (Table 15; Appendix $\mathrm{H}$ ). In addition, there were 18 animals with gubernaculum not present, 12 of which had a bilateral finding. Epididymal anomalies, such as agenesis, are typically seen concomitant with other abnormalities in organs that are developed from Wolffian ducts. Agenesis of all or part of the epididymis (unilateral or bilateral) was observed only in rats in the 10,000 ppm group. Incomplete separation of the prepuce (approximately 14\%), cleft phallus (approximately $6 \%$ ), and/or cleft prepuce (2\%) was observed in 10,000 ppm males relative to no incidences in control rats (Table 15).

Limited genitourinary abnormalities were associated with DEHP exposure in female rats. A greater incidence of vaginal nonpatency (approximately 10\%) occurred in 10,000 ppm females; in contrast a similar finding was not found in any other exposure group. Additionally, observations of cleft phallus were noted in both the 3,000 ppm (approximately 4\%) and 10,000 ppm (approximately $2 \%$ ) females.

Table 15. Summary of Gross Lesions in the Reproductive Tract of Male and Female Rats in the Perinatal and Postweaning Two-year Feed Study of Di(2-ethylhexyl) Phthalate

\begin{tabular}{lccccc}
\hline & $\mathbf{0 ~ p p m}$ & $\mathbf{3 0 0} \mathbf{~ p p m}$ & $\mathbf{1 , 0 0 0} \mathbf{~ p p m}$ & $\mathbf{3 , 0 0 0} \mathbf{~ p p m}$ & $\mathbf{1 0 , 0 0 0} \mathbf{~ p p m}$ \\
\hline Male & & & & & \\
\hline Testis ${ }^{\text {a,b }}$ & 49 & 49 & $50^{\mathrm{c}}$ & 50 & 49 \\
Size, small & $2^{* *}(2)^{\mathrm{e}}$ & $2(2)$ & $4(4)$ & $2(2)$ & $45^{* *}(23)$ \\
Size, enlarged (or swelling) & 0 & 0 & 0 & 0 & $1(1)$ \\
Fluid or blood filled & 0 & $1(1)$ & $1(1)$ & $1(1)$ & $1(1)$ \\
Left, abdominal, undescended & $1^{* *}(1)$ & 0 & 0 & 0 & $13^{*}(10)$ \\
Left, inguinal, undescended & $0^{*}$ & $1(1)$ & $1(1)$ & 0 & $4(4)$ \\
Right, abdominal, undescended & $1^{* *}(1)$ & 0 & 0 & 0 & $18^{* *}(14)$ \\
$\begin{array}{l}\text { Right, inguinal, undescended } \\
\text { Right or left; abdominal; }\end{array}$ & 0 & $1(1)$ & $1(1)$ & 0 & $3(3)$ \\
undescended & $1^{* *}(1)$ & 0 & 0 & 0 & $19^{* *}(14)$ \\
$\begin{array}{l}\text { Right or left; inguinal; } \\
\text { undescended }\end{array}$ & $0^{*}$ & $1(1)$ & $1(1)$ & 0 & $4(4)$ \\
\hline
\end{tabular}


Di(2-ethylhexyl) Phthalate, NTP TR 601

\begin{tabular}{|c|c|c|c|c|c|}
\hline & 0 ppm & 300 ppm & $1,000 \mathrm{ppm}$ & 3,000 ppm & 10,000 ppm \\
\hline $\begin{array}{l}\text { Right or left; abdominal or } \\
\text { inguinal; undescended }\end{array}$ & $1 * *(1)$ & $1(1)$ & $1(1)$ & 0 & $23 * *(16)$ \\
\hline Right, not present & 0 & 0 & 0 & 0 & $1(1)$ \\
\hline Cranial suspensory ligament & $0 * *$ & 0 & 0 & 0 & $5(4)$ \\
\hline Epididymis $^{\mathrm{a}, \mathrm{b}}$ & 49 & 49 & $50^{\mathrm{c}}$ & 50 & 49 \\
\hline Size, small & $0 * *$ & 0 & $2(2)$ & 0 & $14 * *(12)$ \\
\hline Left, caput, agenesis & 0 & 0 & 0 & 0 & $1(1)$ \\
\hline Left, corpus, agenesis & 0 & 0 & 0 & 0 & $1(1)$ \\
\hline $\begin{array}{l}\text { Left, corpus, cauda, or caput, } \\
\text { agenesis/not present }\end{array}$ & 0 & 0 & 0 & 0 & $1(1)$ \\
\hline Right, caput, agenesis & $0 * *$ & 0 & 0 & 0 & $4(4)$ \\
\hline Right, cauda, agenesis & 0 & 0 & 0 & 0 & $2(2)$ \\
\hline Right, corpus, agenesis & 0 & 0 & 0 & 0 & $2(2)$ \\
\hline $\begin{array}{l}\text { Right, corpus, cauda, or caput, } \\
\text { agenesis/not present }\end{array}$ & $0 * *$ & 0 & 0 & 0 & $6(5)$ \\
\hline Right or left, caput, agenesis & $0 * *$ & 0 & 0 & 0 & $4(4)$ \\
\hline Right or left, cauda, agenesis & 0 & 0 & 0 & 0 & $2(2)$ \\
\hline Right or left, corpus, agenesis & $0^{*}$ & 0 & 0 & 0 & $3(3)$ \\
\hline $\begin{array}{l}\text { Right or left, corpus, cauda, or } \\
\text { caput, agenesis/not present }\end{array}$ & $0 * *$ & 0 & 0 & 0 & $6(5)$ \\
\hline LABC Muscle & 50 & 49 & 50 & 50 & 48 \\
\hline Size, small & 0 & 0 & 0 & 0 & $2(2)$ \\
\hline Cowper's Glands & 50 & 49 & 50 & 50 & 47 \\
\hline Left, size, small & 0 & 0 & 0 & 0 & $1(1)$ \\
\hline Right, size, small & 0 & 0 & 0 & 0 & $1(1)$ \\
\hline Prostate Glands & 50 & 49 & 50 & 50 & 47 \\
\hline Size, small & 0 & 0 & 0 & 0 & $1(1)$ \\
\hline $\begin{array}{l}\text { Seminal Vesicles/Coagulating } \\
\text { Glands }\end{array}$ & 50 & 49 & 50 & 50 & 47 \\
\hline Size, small & $1^{* *}(1)$ & 0 & $1(1)$ & $1(1)$ & $8(7)$ \\
\hline Phallus ${ }^{\mathrm{a}, \mathrm{b}}$ & 50 & 49 & 49 & 50 & 49 \\
\hline Size, small & $0^{*}$ & 0 & 0 & 0 & $3(3)$ \\
\hline Cleft & $0^{*}$ & 0 & 0 & 0 & $3(3)$ \\
\hline Prepuce ${ }^{\mathrm{a}, \mathrm{b}}$ & 50 & 49 & 50 & 50 & 49 \\
\hline Cleft & 0 & 0 & 0 & 0 & $1(1)$ \\
\hline Incomplete preputial separation & $0 * *$ & 0 & 0 & 0 & $7 *(7)$ \\
\hline Gubernaculum ${ }^{\mathrm{a}, \mathrm{b}}$ & 47 & 49 & $49^{c}$ & 50 & 41 \\
\hline Left, not present & $0 * *$ & 0 & 0 & 0 & $15^{* *}(12)$ \\
\hline Right, not present & $0 * *$ & 0 & 0 & 0 & $15^{* *}(12)$ \\
\hline Right or left, not present & $0 * *$ & 0 & 0 & 0 & $18 * *(14)$ \\
\hline Gubernaculum Length ${ }^{\mathrm{f}, \mathrm{g}}$ & & & & & \\
\hline
\end{tabular}




\begin{tabular}{lccccc}
\hline & $\mathbf{0 ~ p p m}$ & $\mathbf{3 0 0} \mathbf{~ p p m}$ & $\mathbf{1 , 0 0 0} \mathbf{~ p p m}$ & $\mathbf{3 , 0 0 0} \mathbf{~ p p m}$ & $\mathbf{1 0 , 0 0 0} \mathbf{~ p p m}$ \\
\hline Left, length $(\mathrm{mm})$ & $15.32 \pm 0.69$ & $15.60 \pm 0.63$ & $14.50 \pm 0.52$ & $15.64 \pm 0.58$ & $20.91 \pm 1.91$ \\
& $47(25)^{\mathrm{h}}$ & $49(25)$ & $49(25)$ & $50(25)$ & $24(17)^{\mathrm{i}}$ \\
Right, length $(\mathrm{mm})$ & $15.60 \pm 0.61^{*}$ & $15.46 \pm 0.57$ & $14.34 \pm 0.52$ & $15.62 \pm 0.44$ & $24.22 \pm 2.61^{* *}$ \\
& $47(25)$ & $49(25)$ & $48(25)$ & $50(25)$ & $24(16)$ \\
\hline Female & & & & & \\
Vagina $^{\mathrm{b}}$ & 50 & 50 & 50 & 50 & 48 \\
Not patent $_{\text {Phallus }}$ & $0 *$ & 0 & 0 & 0 & $5(3)$ \\
Cleft $^{\mathrm{b}}$ & 50 & 50 & 50 & 50 & 48 \\
\hline
\end{tabular}

Statistical significance for an exposure group indicates a significant pairwise test compared to the vehicle control group. Statistical significance for the vehicle control group indicates a significant trend test.

$*$ Statistically significant at $\mathrm{p} \leq 0.05 ; * \mathrm{p} \leq 0.01$.

$\mathrm{LABC}=$ levator ani/bulbocavernosus.

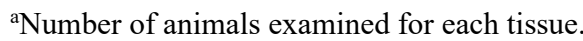

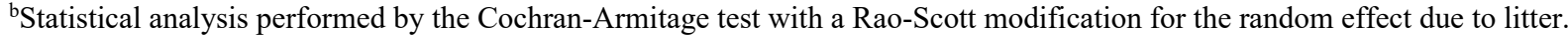
All trend and pairwise $\mathrm{p}$ values are reported as one-sided.

${ }^{\mathrm{c}}$ One animal in the 1,000 ppm group was not examined for right-sided gross lesion in this tissue.

${ }^{\mathrm{d} N u m b e r}$ of animals affected given for each observation.

${ }^{e}$ Number of litters with observations shown in parentheses for $F_{1}$ animals. $F_{1}$ litter incidence based on the number of $F_{0}$ dams.

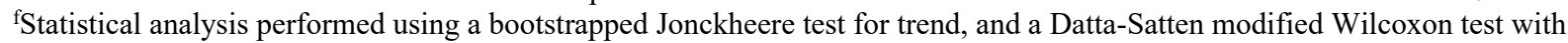

Hommel adjustment for pairwise comparisons.

gData are presented as mean \pm standard error.

${ }^{h} \mathrm{n}=$ number of animals examined (number of litters represented).

${ }^{\mathrm{i}} 15$ animals from the $10,000 \mathrm{ppm}$ group were excluded from statistical analysis due to at least one (left or right) of the gubernaculum observations listed as "not present." An additional two animals from the 10,000 ppm group were excluded from statistical analysis with values listed as "within normal limits," and eight animals had no data collected.

Testis: There were significant increases in germinal epithelium degeneration (includes bilateral), interstitial cell hyperplasia (includes bilateral), and seminiferous tubule dysgenesis (includes bilateral) in the 10,000 ppm group compared to the control group (Table 16). Two of the occurrences of seminiferous tubule dysgenesis were bilateral (Table 16; Appendix H). Germinal epithelium degeneration was recorded when one or more of the following features was present in tubules not adjacent to the rete testis: tubular vacuolation, partial depletion of germ cells, degenerating (multinucleated or apoptotic) germ cells, disordered arrangement of the germ cell layers, or seminiferous tubules completely devoid of germ cells (atrophy) and lined only by Sertoli cells. Germinal epithelium degeneration was scored for severity on a four-grade scale: minimal (grade 1), up to $25 \%$ of at least one testis involved; mild (grade 2), $26 \%$ to $50 \%$ of at least one testis involved; moderate (grade 3 ), $51 \%$ to $75 \%$ of at least one testis involved; and marked (grade 4), rare to no normal seminiferous tubules in either testis were present (i.e., remaining seminiferous tubules solely lined by Sertoli cells).

Interstitial cell hyperplasia, defined as focal aggregates of Leydig cells, was scored using a fourgrade scale: minimal (grade 1), when only a thin rim of interstitial cells or a cluster of cells onefourth the size of a normal seminiferous tubule was present; mild (grade 2), when several such areas were present or one cluster was present that was one-half the size of a normal seminiferous tubule; moderate (grade 3), when a cluster three-fourths the size of a normal seminiferous tubule was present; and marked (grade 4), when a cluster of interstitial cells approached the diameter of a normal seminiferous tubule. The interstitial cells involved were frequently elongated and flattened in profile. Interstitial cell adenomas were characterized by regions of increased 
interstitial cells, described as mostly uniform polyhedral cells with abundant eosinophilic, finely granular, or vacuolated cytoplasm, which exceeded the diameter of three seminiferous tubules. Circumferential compression of adjacent seminiferous tubules was observed occasionally.

Seminiferous tubule dysgenesis only occurred in the highest exposure concentration group $(10,000 \mathrm{ppm})$ and was characterized by seminiferous tubules that were misshapen and contorted, lined by only Sertoli cells, surrounded by a thickened basement membrane, and often accompanied by Leydig cell aggregates (Figure 6). The dysgenesis lesions were commonly associated with an undescended testis but were also identified in scrotal testes. Three of the 10 animals with seminiferous tubule dysgenesis died early, each on day 3 of the study (two were 24 days old and one was 23 days old), and all seminiferous tubule dysgenesis lesions were focal. Focal lesion locations were variable, sometimes being present in sections that did not include the rete testis.

Epididymis: There were significant increases in the incidences of epididymis hypospermia (includes bilateral) in the 10,000 ppm group compared to the control group (Table 16). Epididymis hypospermia was characterized by a reduced density of sperm in the lumen of the epididymal duct, often accompanied by luminal cell debris. It was scored using a four-grade scale: minimal (grade 1), 25\% to 50\% reduction of spermatozoa; mild (grade 2), $51 \%$ to $66 \%$ reduction; moderate (grade 3), $67 \%$ to $80 \%$ reduction; and marked (grade 4 ), $81 \%$ to $100 \%$ reduction. 
Table 16. Incidences of Neoplastic and Nonneoplastic Lesions of the Testis and Epididymis in Male Rats in the Perinatal and Postweaning Two-year Feed Study of Di(2-ethylhexyl) Phthalate

\begin{tabular}{|c|c|c|c|c|c|}
\hline & O ppm & 300 ppm & 1,000 ppm & 3,000 ppm & $10,000 \mathrm{ppm}$ \\
\hline $\mathrm{n}^{\mathrm{a}}$ & 49 & 49 & 50 & 50 & 49 \\
\hline \multicolumn{6}{|l|}{ Testis } \\
\hline $\begin{array}{l}\text { Germinal epithelium, degeneration } \\
\text { (includes bilateral), }\end{array}$ & $16^{* *}(1.6)^{\mathrm{d}}$ & $25(1.6)$ & $21(2.0)$ & $21(1.5)$ & $44 * *(4.0)$ \\
\hline $\begin{array}{l}\text { Interstitial cell, hyperplasia, focal } \\
\text { (includes bilateral) }\end{array}$ & $4 * *(2.0)$ & $3(2.0)$ & $6(2.0)$ & $5(1.4)$ & $30 * *(2.3)$ \\
\hline $\begin{array}{l}\text { Seminiferous tubule, dysgenesis } \\
\text { (includes bilateral) }\end{array}$ & $0 * *$ & 0 & 0 & 0 & $10 *(1.6)$ \\
\hline \multicolumn{6}{|l|}{ Epididymis } \\
\hline Hypospermia (includes bilateral) & $4 * *(2.3)$ & $5(3.4)$ & $12(2.8)$ & $8(2.4)$ & $43^{* *}(4.0)$ \\
\hline \multicolumn{6}{|l|}{ Testis } \\
\hline \multicolumn{6}{|l|}{ Interstitial cell, adenoma ${ }^{\mathrm{e}}$} \\
\hline Overall rate ${ }^{f}$ & $3 / 49(6 \%)$ & $1 / 49(2 \%)$ & $3 / 50(6 \%)$ & $5 / 50(10 \%)$ & $5 / 49(10 \%)$ \\
\hline Rate per litters ${ }^{\mathrm{g}}$ & $3 / 25(12 \%)$ & $1 / 25(4 \%)$ & $3 / 25(12 \%)$ & $5 / 25(20 \%)$ & $4 / 25(16 \%)$ \\
\hline Adjusted rate ${ }^{\mathrm{h}}$ & $7.9 \%$ & $2.4 \%$ & $6.4 \%$ & $11.2 \%$ & $14.1 \%$ \\
\hline Rao-Scott-adjusted Poly- 3 test $\mathrm{t}^{\mathrm{i}}$ & $\mathrm{p}=0.097$ & $\mathrm{p}=0.295 \mathrm{~N}$ & $\mathrm{p}=0.526 \mathrm{~N}$ & $\mathrm{p}=0.461$ & $\mathrm{p}=0.330$ \\
\hline
\end{tabular}

Statistical significance for an exposure group indicates a significant pairwise test compared to the vehicle control group.

Statistical significance for the vehicle control group indicates a significant trend test.

*Significantly different $(\mathrm{p} \leq 0.05)$ from the control group by the Rao-Scott-adjusted Poly-3 test; **p $\leq 0.01$.

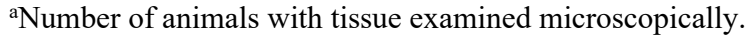

${ }^{b}$ Number of animals with lesion.

'Incidence reported is the combination of unilateral and bilateral lesions.

${ }^{\mathrm{d}}$ Average severity grade of lesions in affected animals: $1=$ minimal, $2=$ mild, $3=$ moderate, $4=$ marked.

${ }^{\mathrm{e}}$ Historical control incidence for all routes of 2-year studies (mean \pm standard deviation): $19 / 487(4.06 \% \pm 4.36 \%)$;

range: $0 \%$ to $14 \%$.

fNumber of animals with neoplasm per number of animals necropsied.

gNumber of litters with neoplasm-bearing animals per number of litters examined at site.

hPoly-3 estimated neoplasm incidence after adjustment for intercurrent mortality.

${ }^{i}$ Beneath the control incidence is the $p$ value associated with the trend test. Beneath the exposed group incidence are the $p$ values corresponding to pairwise comparisons between the control group and that exposed group. The Rao-Scott test adjusts the Poly-3 test (which accounts for differential mortality in animals that do not reach study termination) for within-litter correlation. A negative trend or a lower incidence in an exposure group is indicated by $\mathrm{N}$. 

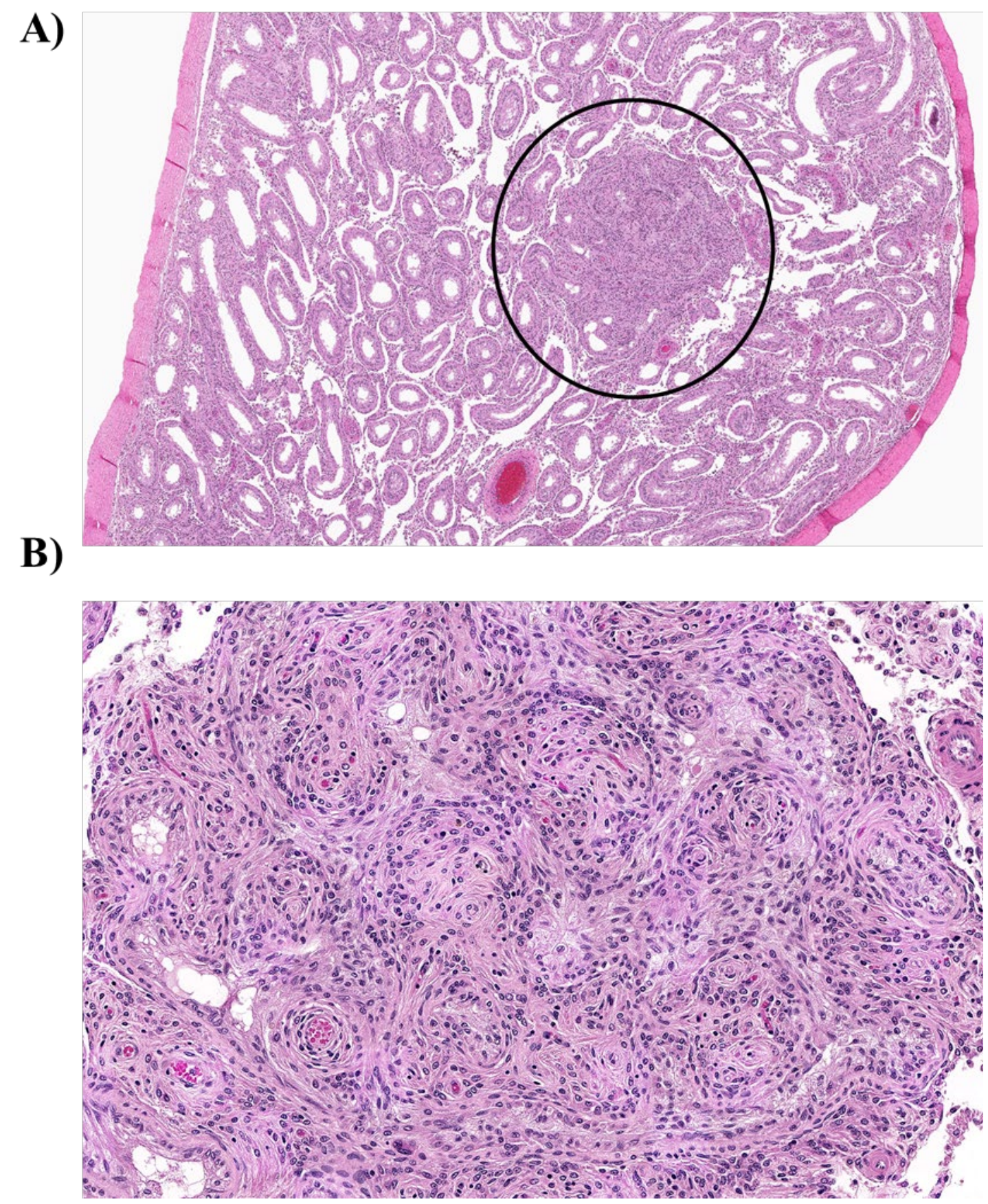

Figure 6. Seminiferous Tubule Dysgenesis in a Male Rat Exposed to Di(2-ethylhexyl) Phthalate in the Perinatal and Postweaning Two-year Feed Study (H\&E)

This image highlights the misshapen, convoluted, and anastomosing tubules in a male rat exposed to 10,000 ppm DEHP. Panel B is a higher magnification $(20 \mathrm{x})$ of the region within the circle of panel $\mathrm{A}(4 \times$ magnification).

Uterus: A positive trend with increasing exposure concentration in uterus endometrium adenocarcinoma and uterus adenoma, adenocarcinoma, squamous cell carcinoma, or squamous cell papilloma (combined) (Table 17). Uterus endometrium adenocarcinoma typically is poorly 
circumscribed and invades the myometrium. The neoplastic epithelial cells form solid nests, cords, papillary, or acinar structures that are within, or supported by, stroma.

Table 17. Incidences of Neoplastic Lesions of the Uterus (Including Cervix) in Female Rats in the Perinatal and Postweaning Two-year Feed Study of Di(2-ethylhexyl) Phthalate

\begin{tabular}{|c|c|c|c|c|c|}
\hline & 0 ppm & 300 ppm & $1,000 \mathrm{ppm}$ & 3,000 ppm & 10,000 ppm \\
\hline $\mathbf{n}^{\mathbf{a}}$ & 50 & 50 & 50 & 50 & 48 \\
\hline Adenoma $^{\mathrm{b}, \mathrm{c}}$ & 0 & 1 & 0 & 0 & 0 \\
\hline \multicolumn{6}{|l|}{ Adenocarcinoma $^{\mathrm{d}}$} \\
\hline Overall rate $\mathrm{e}^{\mathrm{e}}$ & $3 / 50(6 \%)$ & $0 / 50(0 \%)$ & $1 / 50(2 \%)$ & $3 / 50(6 \%)$ & $6 / 48(13 \%)$ \\
\hline Rate per litters ${ }^{\mathrm{f}}$ & $3 / 25(12 \%)$ & $0 / 25(0 \%)$ & $1 / 25(4 \%)$ & $3 / 25(12 \%)$ & $6 / 25(24 \%)$ \\
\hline Adjusted rate ${ }^{g}$ & $7 \%$ & $0 \%$ & $2.4 \%$ & $7 \%$ & $16.4 \%$ \\
\hline Rao-Scott-adjusted Poly-3 test ${ }^{\mathrm{h}}$ & $\mathrm{p}=0.008$ & $\mathrm{p}=0.147 \mathrm{~N}$ & $\mathrm{p}=0.325 \mathrm{~N}$ & $\mathrm{p}=0.653 \mathrm{~N}$ & $\mathrm{p}=0.184$ \\
\hline $\begin{array}{l}\text { Squamous Cell Carcinoma } \\
\text { (Includes Multiple) }\end{array}$ & 0 & 1 & 0 & 0 & 1 \\
\hline Squamous Cell Papilloma ${ }^{\mathrm{j}}$ & 0 & 0 & 0 & 1 & 0 \\
\hline \multicolumn{6}{|c|}{ Adenoma, Adenocarcinoma, Squamous Cell Carcinoma, or Squamous Cell Papilloma (Combined) ${ }^{\mathrm{k}}$} \\
\hline Overall rate & $3 / 50(6 \%)$ & $1 / 50(2 \%)$ & $1 / 50(2 \%)$ & $3 / 50(6 \%)$ & $7 / 48(15 \%)$ \\
\hline Rate per litters & $3 / 25(12 \%)$ & $1 / 25(4 \%)$ & $1 / 25(4 \%)$ & $3 / 25(12 \%)$ & $7 / 25(28 \%)$ \\
\hline Adjusted rate & $7 \%$ & $2.4 \%$ & $2.4 \%$ & $7 \%$ & $19 \%$ \\
\hline Rao-Scott-adjusted Poly-3 test & $\mathrm{p}=0.005$ & $\mathrm{p}=0.325 \mathrm{~N}$ & $\mathrm{p}=0.317 \mathrm{~N}$ & $\mathrm{p}=0.651 \mathrm{~N}$ & $\mathrm{p}=0.113$ \\
\hline
\end{tabular}

Statistical significance for an exposure group indicates a significant pairwise test compared to the vehicle control group.

*Significantly different $(\mathrm{p} \leq 0.05)$ from the control group by the Rao-Scott-adjusted Poly-3 test.

aNumber of animals with tissue examined microscopically.

${ }^{b}$ Number of animals with lesion.

${ }^{\mathrm{c}}$ Historical control incidence for all routes of 2-year studies (mean \pm standard deviation): $1 / 350(0.29 \% \pm 0.76 \%)$;

range: $0 \%$ to $2 \%$.

${ }^{\mathrm{d}}$ Historical control incidence: $20 / 350(5.71 \% \pm 3.35 \%)$; range: $2 \%$ to $10 \%$.

${ }^{\mathrm{e} N u m b e r}$ of animals with neoplasm per number of animals necropsied.

f Number of litters with neoplasm-bearing animals per number of litters examined at site.

gPoly-3 estimated neoplasm incidence after adjustment for intercurrent mortality.

${ }^{h}$ Beneath the control incidence is the $p$ value associated with the trend test. Beneath the exposed group incidence are the $p$ values corresponding to pairwise comparisons between the control group and that exposed group. The Rao-Scott test adjusts the Poly-3 test (which accounts for differential mortality in animals that do not reach study termination) for within-litter correlation. A negative trend or a lower incidence in an exposure group is indicated by $\mathrm{N}$.

${ }^{i}$ Historical control incidence: $2 / 350(0.57 \% \pm 1.51 \%)$; range: $0 \%$ to $4 \%$.

jHistorical control incidence: $0 / 350$.

${ }^{\mathrm{k}}$ Historical control incidence: $23 / 350(6.57 \% \pm 3.41 \%)$; range: $2 \%$ to $10 \%$.

Kidney: There were significant increases in the incidences of nonneoplastic kidney lesions in male and female DEHP-exposed groups relative to the control groups (Table 18). The incidences of papilla edema and papilla epithelium hyperplasia were significantly increased in the 10,000 ppm males and females relative to control animals; there was a significant increase in the incidence of papilla hemorrhage in the $10,000 \mathrm{ppm}$ males. There were significant increases in the incidence of kidney infarct in the 300 and $10,000 \mathrm{ppm}$ males and the 1,000 and $10,000 \mathrm{ppm}$ females. A significant increase in the incidence of renal tubule cyst was observed in the $10,000 \mathrm{ppm}$ female group compared to that of the control group. A positive trend in the incidence of renal tubule dilation occurred with increasing exposure concentration in the females. 
Papilla edema (Figure 7) occurred only in exposed animals, was present in most males and females exposed to $10,000 \mathrm{ppm}$, and was observed in two females exposed to 1,000 ppm DEHP. Papillary edema affected the kidneys bilaterally and was characterized by expansion of the papillary interstitium by fibrillary amphophilic to pale eosinophilic material. Collecting ducts were often dilated and distorted and lined by a continuous layer of thin attenuated epithelium. The dilatation of collecting ducts sometimes extended to include renal tubules within the medulla and/or cortex. Representative sections were stained with Alcian blue, which identifies glycosaminoglycans (GAGs), and with periodic acid-Schiff (PAS) to evaluate basement membrane integrity. The interstitial material stained positively for Alcian blue, confirming that the material contained GAGs. The basement membranes of vascular structures stained intensely positive for PAS throughout the kidney sections and were not compromised in any areas, indicating they were intact. The renal tubules in the cortex and outer medulla stained uniformly and intensely positive for PAS, but there was an abrupt loss of staining at the junction of the outer and inner medulla. Tubules and collecting ducts within the inner medulla and papilla lacked staining for PAS, indicating disruption of the basement membrane of tubules/ducts within this region.

Papillary hemorrhage occurred in regions of papillary edema (Figure 8). Papilla epithelium hyperplasia was diagnosed when there was thickening and/or variably sized outgrowths (with clear spaces) of the epithelium overlying the renal papilla (Figure 9). Occasionally, these spaces contained eosinophilic material or cells. Although papillary epithelial hyperplasia is commonly associated with advanced chronic progressive nephropathy (CPN), there was no direct correlation with CPN severity in this study.

Kidney infarct consisted of well-demarcated, wedge-shaped regions characterized by renal interstitial fibrosis and depression of the overlying capsule; lesions extended from the capsular surface into the medulla. Infarcts were scored using a four-grade severity scale: minimal (grade 1), $<25 \%$ of renal involvement; mild (grade 2), $25 \%$ to $50 \%$ of renal involvement; moderate (grade 3), $51 \%$ to $75 \%$ of renal involvement; and marked (grade 4 ), $>75 \%$ renal involvement. Renal tubule cysts were characterized by dilated renal tubules lined by cuboidal to thin attenuated epithelial cells. There was a positive trend in renal tubule dilation in females.

Table 18. Incidences of Nonneoplastic Lesions of the Kidney in Male and Female Rats in the Perinatal and Postweaning Two-year Feed Study of Di(2-ethylhexyl) Phthalate

\begin{tabular}{lccccc}
\hline & $\mathbf{0 ~} \mathbf{~ p p m}$ & $\mathbf{3 0 0} \mathbf{~ p p m}$ & $\mathbf{1 , 0 0 0} \mathbf{~ p p m}$ & $\mathbf{3 , 0 0 0} \mathbf{~ p p m}$ & $\mathbf{1 0 , 0 0 0} \mathbf{~ p p m}$ \\
\hline Male & & & & & \\
\hline $\mathbf{n}^{\mathbf{a}}$ & 50 & 49 & 50 & 50 & 49 \\
Papilla, Edema $^{\mathrm{b}}$ & $0^{* *}$ & 0 & 0 & 0 & $39^{* *}(2.2)^{\mathrm{c}}$ \\
Papilla, Hemorrhage & $0^{* *}$ & $1(2.0)$ & 0 & $2(1.0)$ & $12^{* *}(1.7)$ \\
Epithelium, Papilla, Hyperplasia & $9^{* *}(1.3)$ & $4(1.3)$ & $4(1.8)$ & $3(1.0)$ & $17^{*}(1.2)$ \\
Infarct & $2^{* *}(1.0)$ & $10^{*}(1.0)$ & $9(1.0)$ & $7(1.0)$ & $17^{* *}(1.1)$ \\
Renal Tubule, Cyst & 9 & 2 & 4 & 7 & 5 \\
Renal Tubule, Dilation & 0 & 0 & 0 & $2(2.5)$ & 0 \\
\hline
\end{tabular}


Di(2-ethylhexyl) Phthalate, NTP TR 601

\begin{tabular}{lccccc}
\hline & $\mathbf{0 ~} \mathbf{p p m}$ & $\mathbf{3 0 0} \mathbf{~ p p m}$ & $\mathbf{1 , 0 0 0} \mathbf{~ p p m}$ & $\mathbf{3 , 0 0 0} \mathbf{~ p p m}$ & $\mathbf{1 0 , 0 0 0} \mathbf{~ p p m}$ \\
\hline Female & & & & & \\
\hline $\mathbf{n}$ & 50 & 50 & 50 & 50 & 48 \\
Papilla, Edema & $0^{* *}$ & 0 & $2(1.5)$ & 0 & $38^{* *}(1.6)$ \\
Papilla, Hemorrhage & 0 & 0 & 0 & 0 & $2(1.0)$ \\
Epithelium, Papilla, Hyperplasia & $2^{* *}(1.0)$ & $1(3.0)$ & $2(1.0)$ & $4(1.3)$ & $15^{* *}(1.3)$ \\
Infarct & $0^{* *}$ & $3(2.0)$ & $7^{*}(1.1)$ & $5(1.0)$ & $12^{* *}(1.5)$ \\
Renal Tubule, Cyst & $0^{* *}$ & 0 & 2 & 0 & $7 *$ \\
Renal Tubule, Dilation & $0^{*}$ & 0 & 0 & 0 & $3(2.7)$ \\
\hline
\end{tabular}

Statistical significance for an exposure group indicates a significant pairwise test compared to the vehicle control group. Statistical significance for the vehicle control group indicates a significant trend test.

*Statistically significant $(\mathrm{p} \leq 0.05)$ from the vehicle control group by the Rao-Scott-adjusted Poly-3 test; ${ }^{*} \mathrm{p} \leq 0.01$.

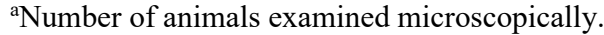

${ }^{b}$ Number of animals with lesion.

${ }^{\mathrm{c}}$ Average severity grade of observed lesion in affected animals: $1=$ minimal; $2=$ mild; $3=$ moderate; $4=$ marked . 
A)

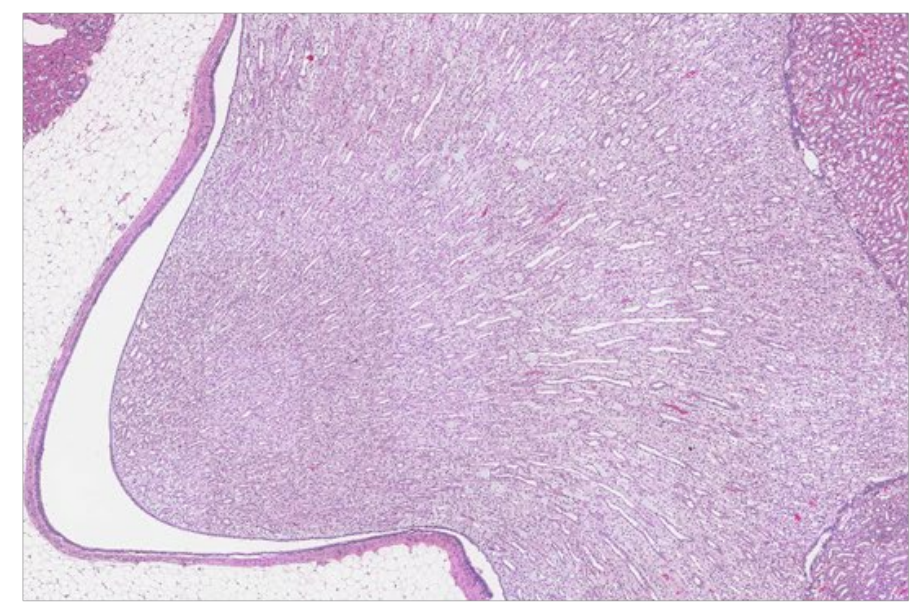

C)

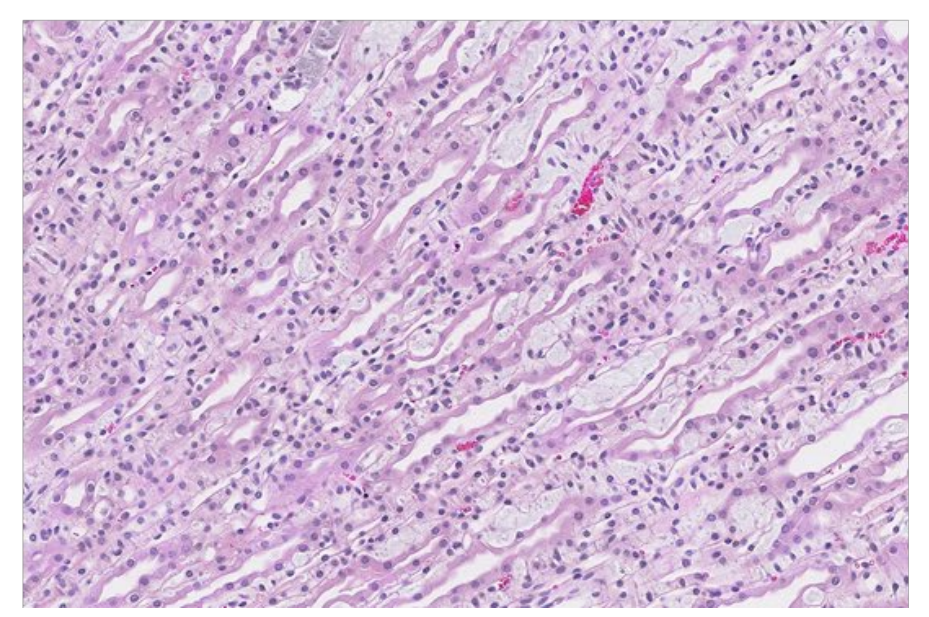

B)

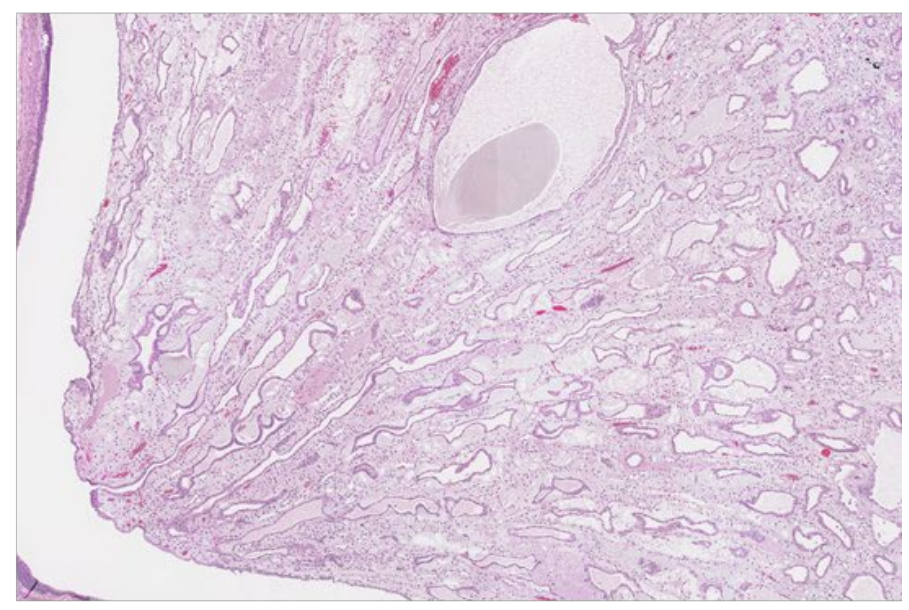

D)

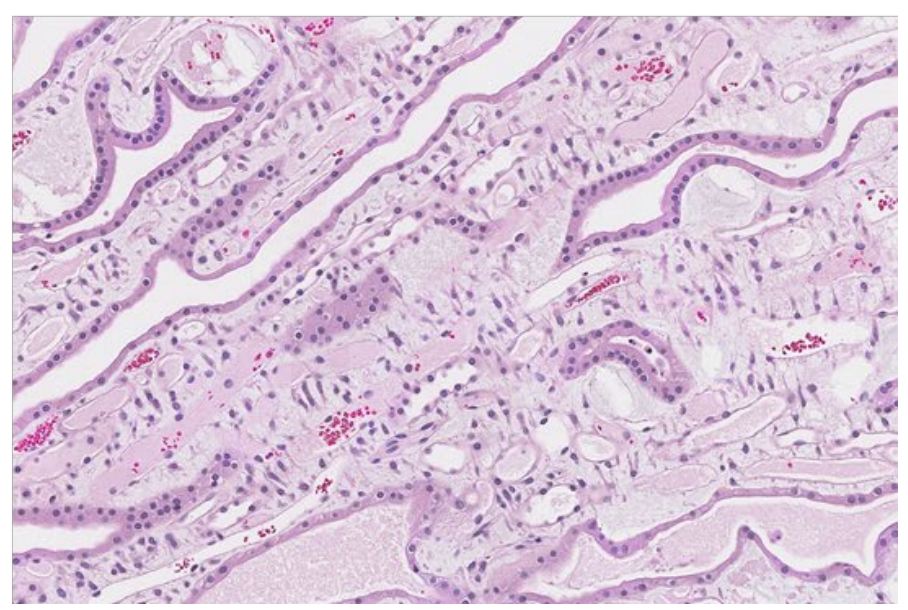

Figure 7. Kidney Papilla Edema in Male and Female Rats Exposed to Di(2-ethylhexyl) Phthalate in the Perinatal and Postweaning Two-year Feed Study (H\&E)

Kidney from a control female rat with normal papilla structure (panel A at $4 \times$ magnification, panel $\mathrm{C}$ at $20 \times$ magnification) compared to kidney from a male 10,000 ppm rat with papillary edema (panel B at $4 \times$ magnification, panel D at $20 \times$ magnification). This lesion was characterized by expansion of the papillary interstitium by fibrillary amphophilic to pale eosinophilic material. Collecting ducts were often dilated, distorted, and lined by a continuous layer of thin attenuated epithelium. 


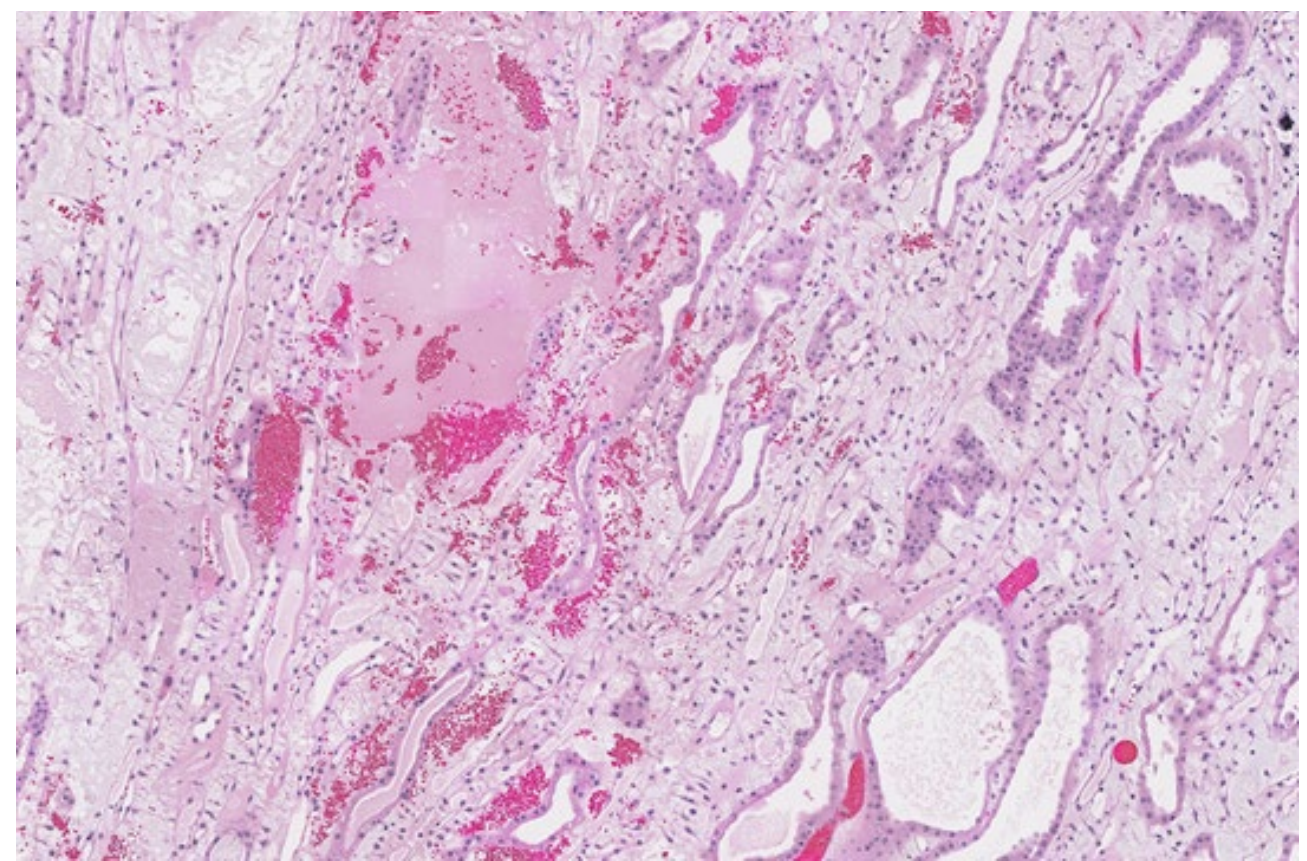

Figure 8. Renal Papillary Hemorrhage in a Male Rat Exposed to Di(2-ethylhexyl) Phthalate in the Perinatal and Postweaning Two-year Feed Study (H\&E)

This image is an example of mild hemorrhage in a kidney from a 1,000 ppm male rat with moderate papillary edema at $10 \times$ magnification.

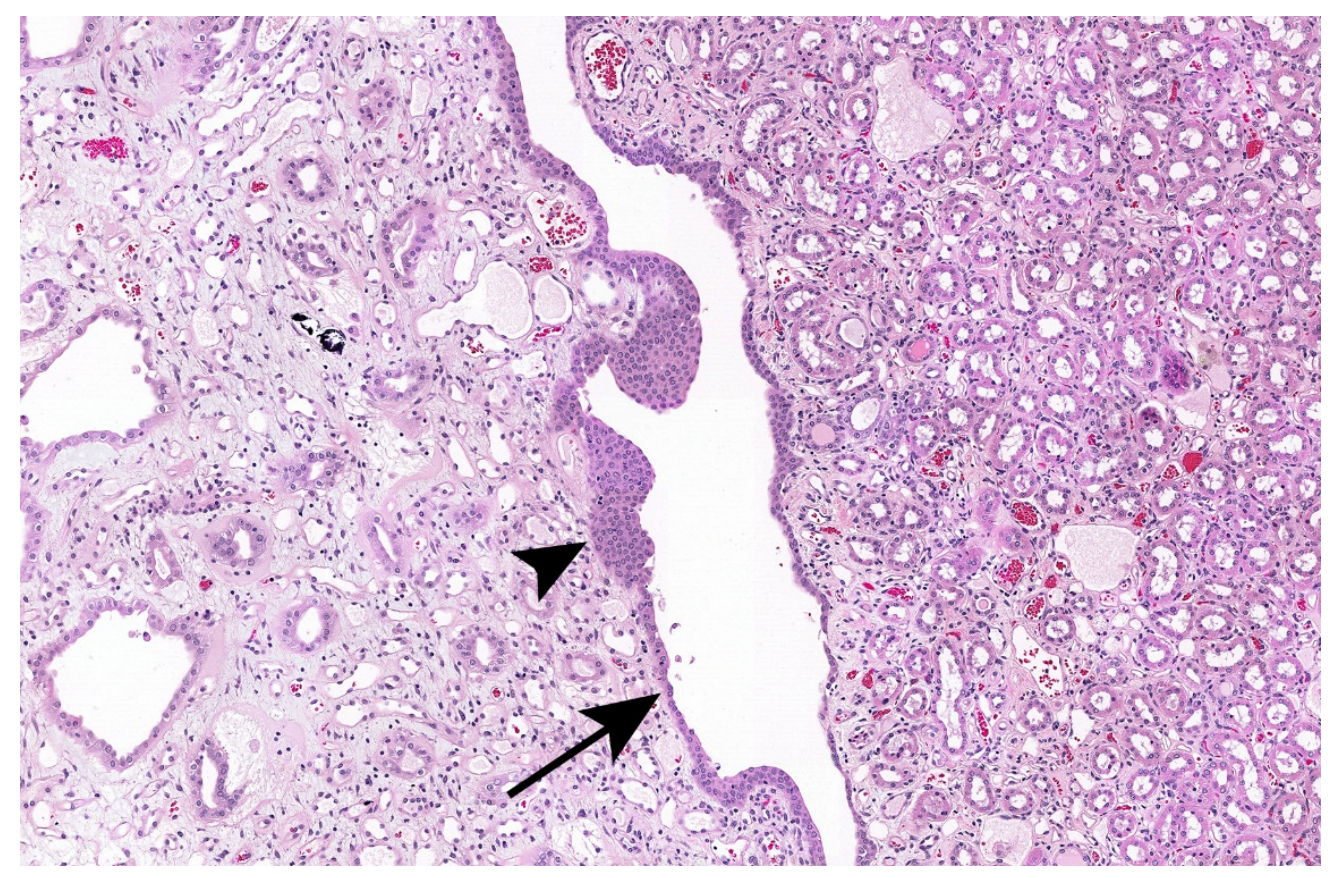

Figure 9. Renal Papillary Epithelium Hyperplasia in a Male Rat Exposed to Di(2-Ethylhexyl) Phthalate in the Perinatal and Postweaning Two-year Feed Study (H\&E)

This image is an example of mild epithelial hyperplasia in a 10,000 ppm male rat with normal low cuboidal lining cells (long arrow) are increased in thickness (arrowhead) at $10 \times$ magnification. 
Heart: A significant increase in the incidences of heart valve fibrosis and heart valve thrombus occurred in the 10,000 ppm males, relative to the control animals (Table 19). Heart valve fibrosis was diagnosed when valves were expanded by fibrous connective tissue that was more densely eosinophilic than the loose lightly basophilic to amphophilic tissue of a normal heart valve (Figure 10). Severity was scored using a four-grade scale: minimal (grade 1), change was barely detectable to thickening of the valve up to double the normal thickness; mild (grade 2), thickening up to three times the normal thickness, involvement of several portions of the valve, or more than one valve up to double the normal thickness; moderate (grade 3), thickening of several regions of the valve or valves, at least one of which was greater than double the normal thickness of a valve at that location; marked (grade 4) valve fibrosis was not diagnosed. Heart valve thrombus was characterized by fibrin, admixed with variable numbers of blood cells, that covered the cardiac valves. Severity grades were assigned according to the following grading scheme: minimal (grade 1), amount of fibrin and cells was less than the thickness of the widest part of the valve; mild (grade 2), layering upon the valves that could occlude approximately $50 \%$ of the valve lumen; moderate (grade 3), lesions that occluded $51 \%$ to $80 \%$ of the valve lumen; marked (grade 4 ), lesions that occluded $\geq 81 \%$ of the valve lumen.

Bone Marrow: There was a significant increase in the incidence of bone marrow hypercellularity in the 3,000 and 10,000 ppm male groups, relative to the control group (Table 19). Bone marrow hypercellularity was characterized by an increase in one or more hematopoietic cell lines, generally with a decrease in adipocytes.

Pituitary Gland: There was a significant increase in the incidence of pars distalis hypertrophy in the 3,000 and 10,000 ppm males compared to control animals (Table 19). Pars distalis hypertrophy was characterized by clusters of cells that were round, with abundant vacuolated amorphous amphophilic or pale eosinophilic cytoplasm and peripherally compressed nuclei, scattered throughout the pars distalis (Figure 11). A severity grade was assigned based on the numbers of affected cells.

Table 19. Incidences of Select Nonneoplastic Lesions of the Heart, Bone Marrow, and Pituitary Gland in Male Rats in the Perinatal and Postweaning Two-year Feed Study of Di(2-ethylhexyl) Phthalate

\begin{tabular}{lccccc}
\hline & $\mathbf{0 ~} \mathbf{~ p p m}$ & $\mathbf{3 0 0} \mathbf{~ p p m}$ & $\mathbf{1 , 0 0 0} \mathbf{~ p p m}$ & $\mathbf{3 , 0 0 0} \mathbf{~ p p m}$ & $\mathbf{1 0 , 0 0 0} \mathbf{~ p p m}$ \\
\hline Heart $^{\mathrm{a}}$ & 50 & 49 & 50 & 50 & 49 \\
Valve, fibrosis $^{\mathrm{b}}$ & $0^{* *}$ & $2(1.0)^{\mathrm{c}}$ & $1(2.0)$ & $3(1.3)$ & $11^{* *}(1.7)$ \\
Valve, thrombus & $0^{* *}$ & 0 & 0 & 0 & $6^{*}(1.8)$ \\
Bone Marrow & 50 & 49 & 50 & 50 & 50 \\
Hypercellularity & $21^{* *}(2.2)$ & $17(1.9)$ & $29(1.9)$ & $34^{*}(1.9)$ & $36^{* *}(2.1)$ \\
Pituitary Gland & 50 & 49 & 50 & 50 & 49 \\
Pars distalis, hypertrophy & $3^{* *}(1.0)$ & $7(1.1)$ & $5(1.0)$ & $15^{* *}(1.3)$ & $37^{* *}(2.4)$ \\
\hline
\end{tabular}

Statistical significance for an exposure group indicates a significant pairwise test compared to the vehicle control group.

Statistical significance for the vehicle control group indicates a significant trend test.

*Statistically significant $(\mathrm{p} \leq 0.05)$ from the vehicle control group by the Rao-Scott-adjusted Poly- 3 test; **p $\leq 0.01$.

${ }^{a}$ Number of animals examined microscopically.

bNumber of animals with lesion.

${ }^{\mathrm{c}}$ Average severity grade of observed lesion in affected animals: $1=$ minimal; $2=$ mild; $3=$ moderate; $4=$ marked. 
A)

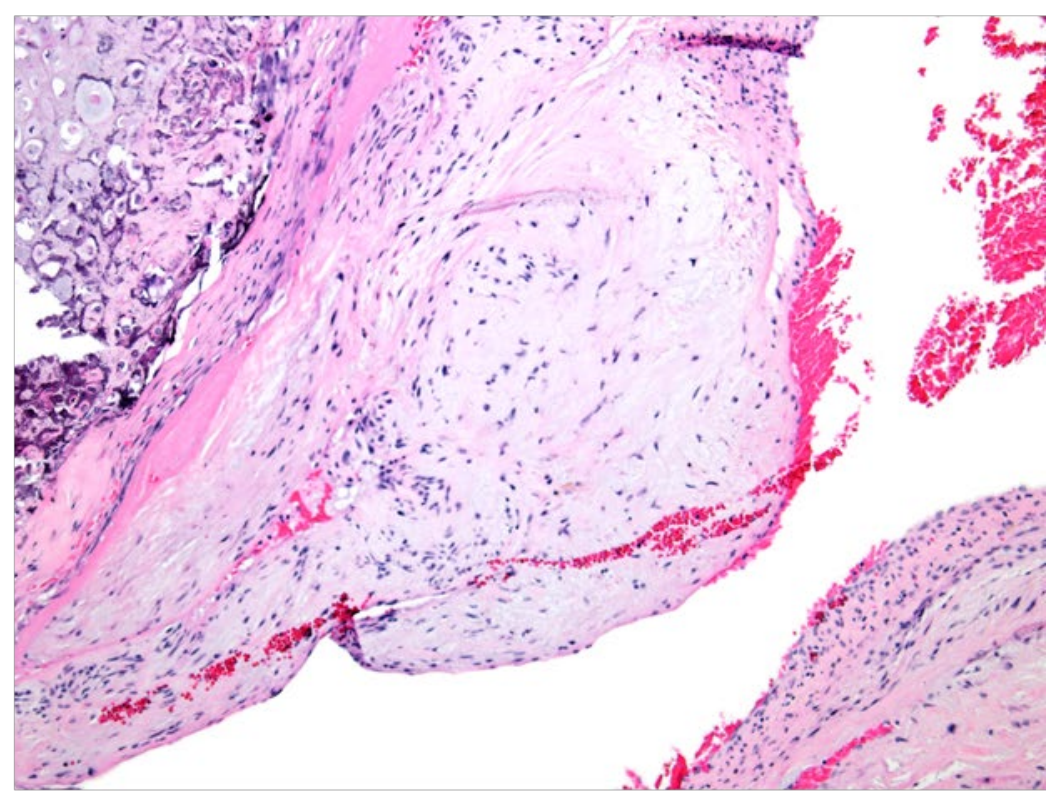

B)

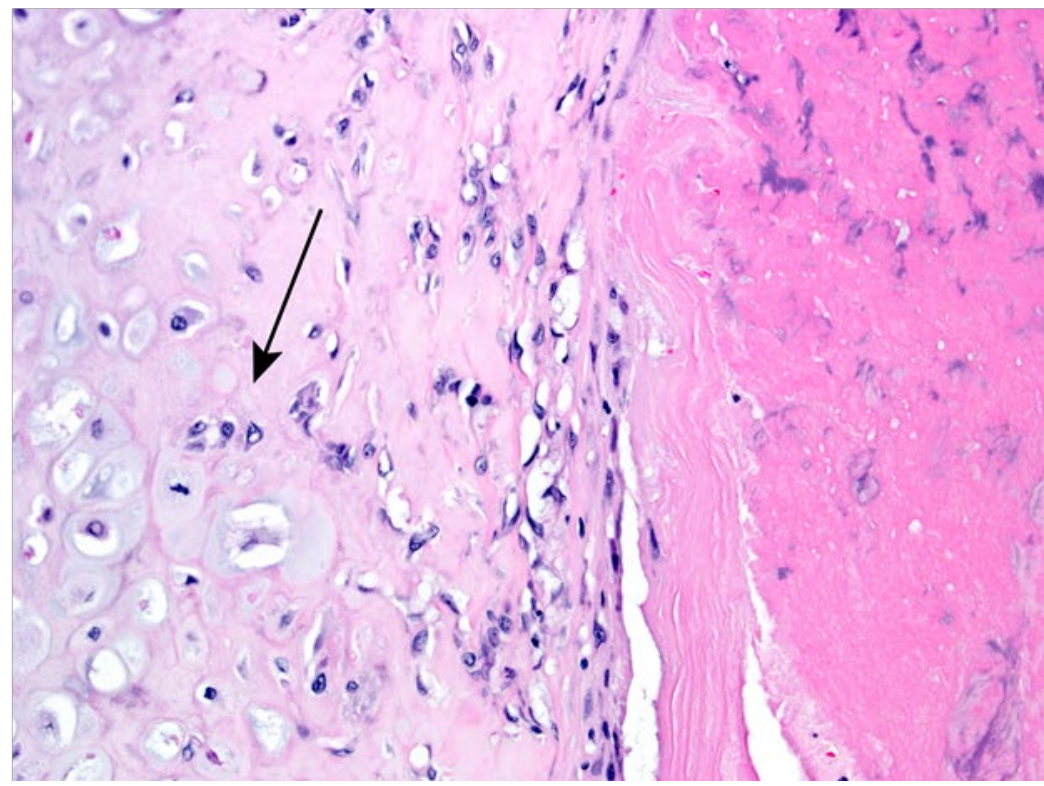

Figure 10. Heart Valve Fibrosis in a Male Rat Exposed to Di(2-Ethylhexyl) Phthalate in the Perinatal and Postweaning Two-year Feed Study (H\&E)

The images show examples of heart valve fibrosis in a 1,000 ppm male at $10 \times$ magnification (A) and 20× magnification (B). The lesion was characterized by valves that were expanded by fibrous connective tissue that was more densely eosinophilic than the loose lightly basophilic to amphophilic tissue of a normal heart valve. Cartilaginous metaplasia (arrow) was sometimes associated with the valvular fibrosis. 
Di(2-ethylhexyl) Phthalate, NTP TR 601

A)

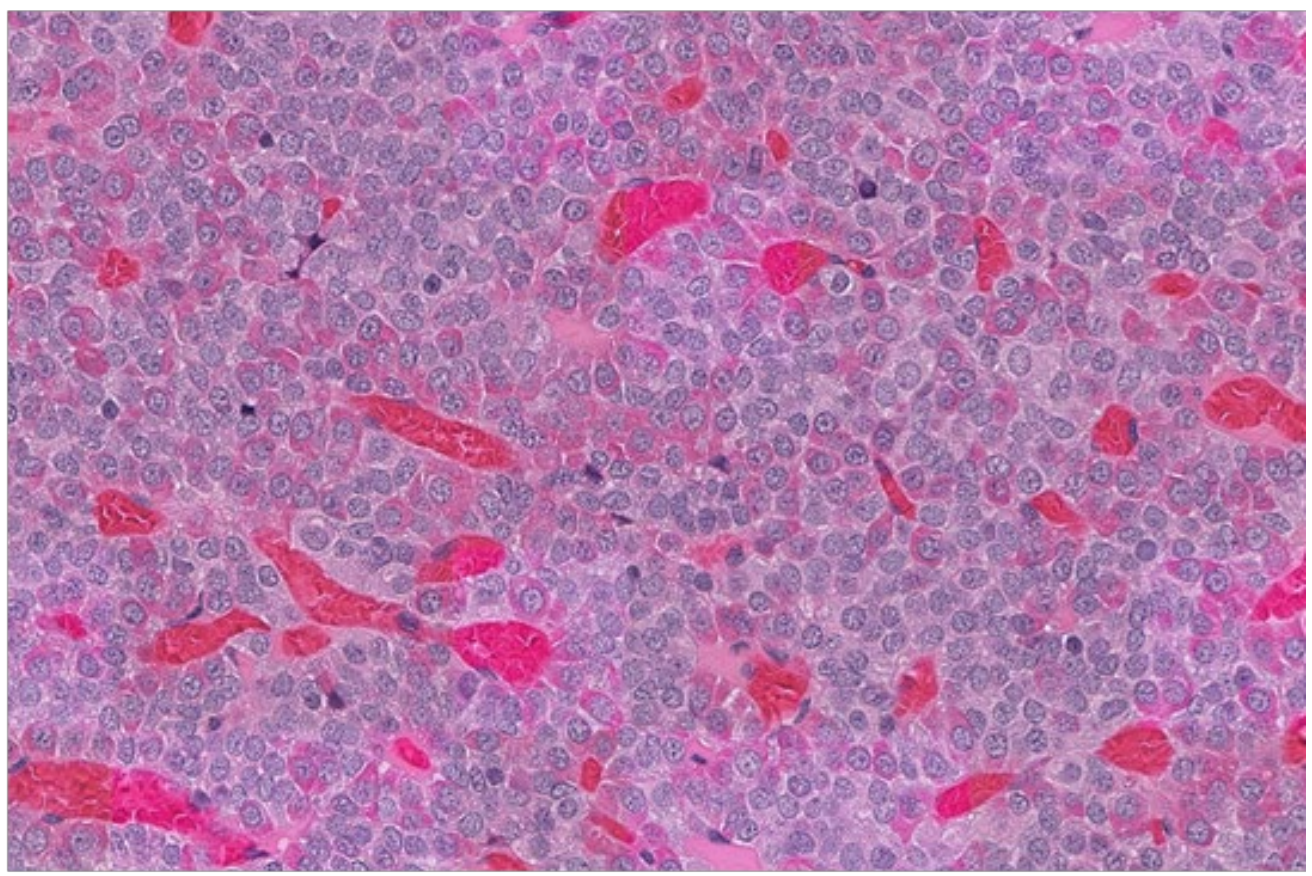

B)

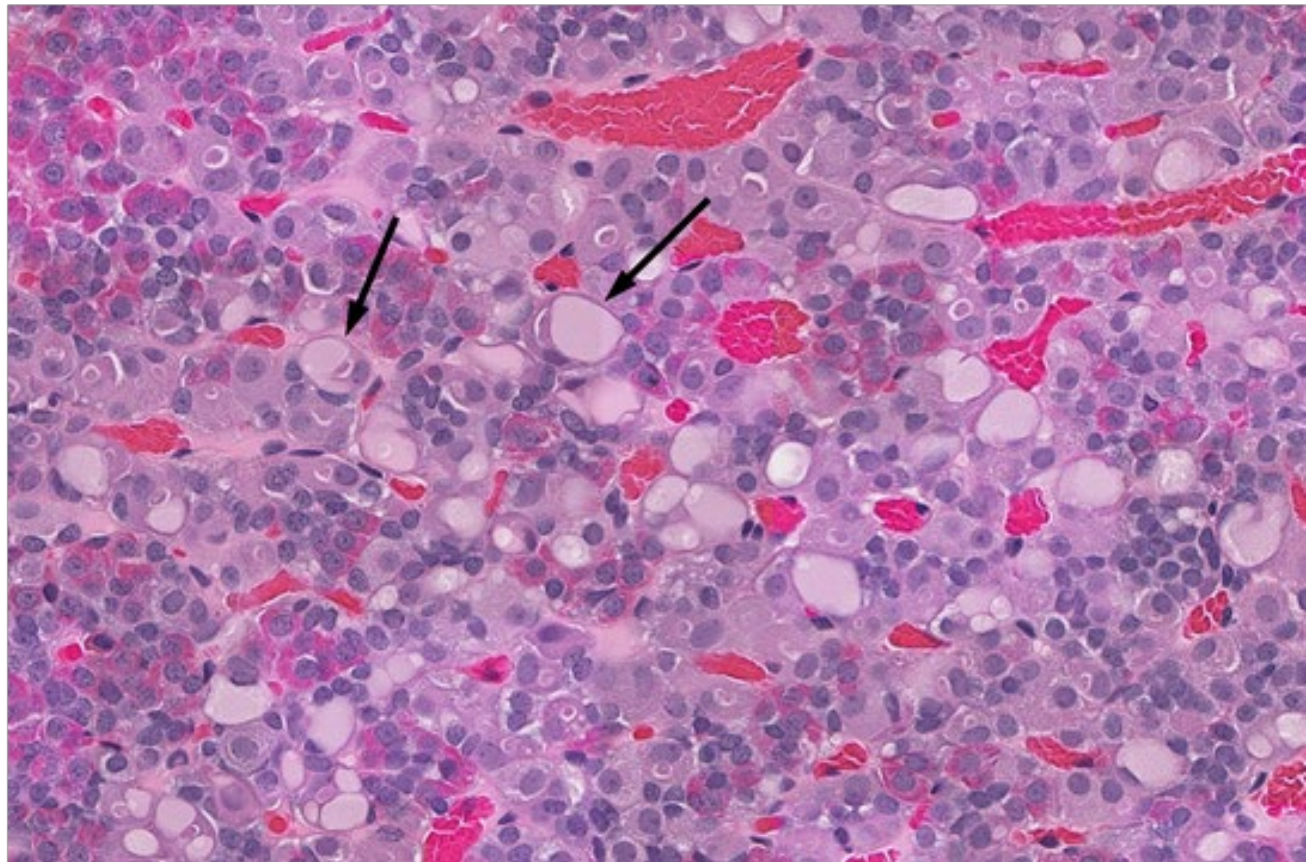

Figure 11. Pituitary Pars Distalis Hypertrophy in Male Rats Exposed to Di(2-ethylhexyl) Phthalate in the Perinatal and Postweaning Two-year Feed Study (H\&E)

The images compare normal pars distalis in a control male (panel A) with one from a 10,000 ppm male that is hypertrophied (panel B) and characterized by clusters of cells that are round, with abundant amorphous amphophilic or pale eosinophilic cytoplasm and peripherally compressed nuclei (arrows) at $40 \times$ magnification. 
The toxicological significance of other lesion findings is unknown as the response was considered either marginal, not dose-responsive, and/or potentially decreased (potentially due to lower body weights) (Appendix H). In males, these lesions included: adrenal cortex focal hyperplasia, adrenal medulla focal hyperplasia, testis polyarteritis nodosa, bilateral testis polyarteritis nodosa, thyroid gland $\mathrm{C}$-cell adenoma, and thyroid gland C-cell adenoma or carcinoma (combined). In females, these lesions included: nose respiratory epithelium hyaline droplet accumulation, ovary atrophy, uterus endometrium squamous metaplasia, mammary gland fibroadenoma, pituitary gland pars distalis, or unspecified site adenoma in females. 


\section{Postweaning-only Study in Rats (Study 2)}

Overall, survival at study termination of male and female rats exposed to DEHP was commensurate with or greater than that of control animals (Table 20; Figure 12). Survival to study termination was significantly increased in $10,000 \mathrm{ppm}$ males (approximately $84 \%$ ) relative to control males (approximately 64\%).

Table 20. Summary of Survival of Male and Female Rats in the Postweaning-only Two-year Feed Study of Di(2-ethylhexyl) Phthalate

\begin{tabular}{|c|c|c|c|c|c|}
\hline & O ppm & 300 ppm & $1,000 \mathrm{ppm}$ & 3,000 ppm & 10,000 ppm \\
\hline \multicolumn{6}{|l|}{ Male } \\
\hline Animals Initially in Study & 50 & 50 & 50 & 50 & 50 \\
\hline Moribund & 4 & 8 & 2 & 8 & 4 \\
\hline Natural Deaths & 14 & 8 & 9 & 7 & 4 \\
\hline $\begin{array}{l}\text { Animals Surviving to Study } \\
\text { Termination }\end{array}$ & 32 & $34^{\mathrm{a}}$ & 39 & 35 & 42 \\
\hline $\begin{array}{l}\text { Percent Probability of Survival } \\
\text { at End of Study }\end{array}$ & 64.0 & 68.0 & 78.0 & 70.0 & 84.0 \\
\hline Mean Survival (Days) ${ }^{\mathrm{c}}$ & 675 & 692 & 706 & 696 & 711 \\
\hline Survival Analysis ${ }^{\mathrm{d}}$ & $\mathrm{p}=0.061 \mathrm{~N}$ & $\mathrm{p}=0.692 \mathrm{~N}$ & $\mathrm{p}=0.139 \mathrm{~N}$ & $\mathrm{p}=0.553 \mathrm{~N}$ & $\mathrm{p}=0.037 \mathrm{~N}$ \\
\hline \multicolumn{6}{|l|}{ Female } \\
\hline Animals Initially in Study & 50 & 50 & 50 & 50 & 50 \\
\hline Moribund & 9 & 10 & 13 & 9 & 6 \\
\hline Natural Deaths & 8 & 6 & 4 & 7 & 12 \\
\hline $\begin{array}{l}\text { Animals Surviving to Study } \\
\text { Termination }\end{array}$ & 33 & 34 & $33^{\mathrm{e}}$ & 34 & $32^{\mathrm{f}}$ \\
\hline $\begin{array}{l}\text { Percent Probability of Survival } \\
\text { at End of Study }\end{array}$ & 66.0 & 68.0 & 66.0 & 68.0 & 64.0 \\
\hline Mean Survival (Days) & 691 & 668 & 678 & 700 & 684 \\
\hline Survival Analysis & $\mathrm{p}=0.834$ & $\mathrm{p}=1.000 \mathrm{~N}$ & $\mathrm{p}=1.000$ & $\mathrm{p}=0.932 \mathrm{~N}$ & $\mathrm{p}=0.904$ \\
\hline $\begin{array}{l}\text { Includes one animal that died natur } \\
\text { Kaplan-Meier determinations. } \\
\text { Mean of all deaths (uncensored, ce } \\
\text { The result of the life-table trend tes } \\
\text { the vehicle control group are in the } \\
\text { by N. } \\
\text { Includes one animal that died natur } \\
\text { Includes one animal that died natur }\end{array}$ & $\begin{array}{l}\text { y during the las } \\
\text { red, and study } \\
\text { in the vehicle } \\
\text { osed group col } \\
\text { y and one anim } \\
\text { y during the las }\end{array}$ & $\begin{array}{l}\text { eek of the study } \\
\text { nination). } \\
\text { trol column, an } \\
\text { ns. A negative }\end{array}$ & $\begin{array}{l}\text { results of the } \\
\text { or lower mor }\end{array}$ & $\begin{array}{l}\text { table pairwis } \\
\text { in an expost } \\
\text { ee last week o }\end{array}$ & $\begin{array}{l}\text { aparisons with } \\
\text { oup is indicated } \\
\text { study. }\end{array}$ \\
\hline
\end{tabular}



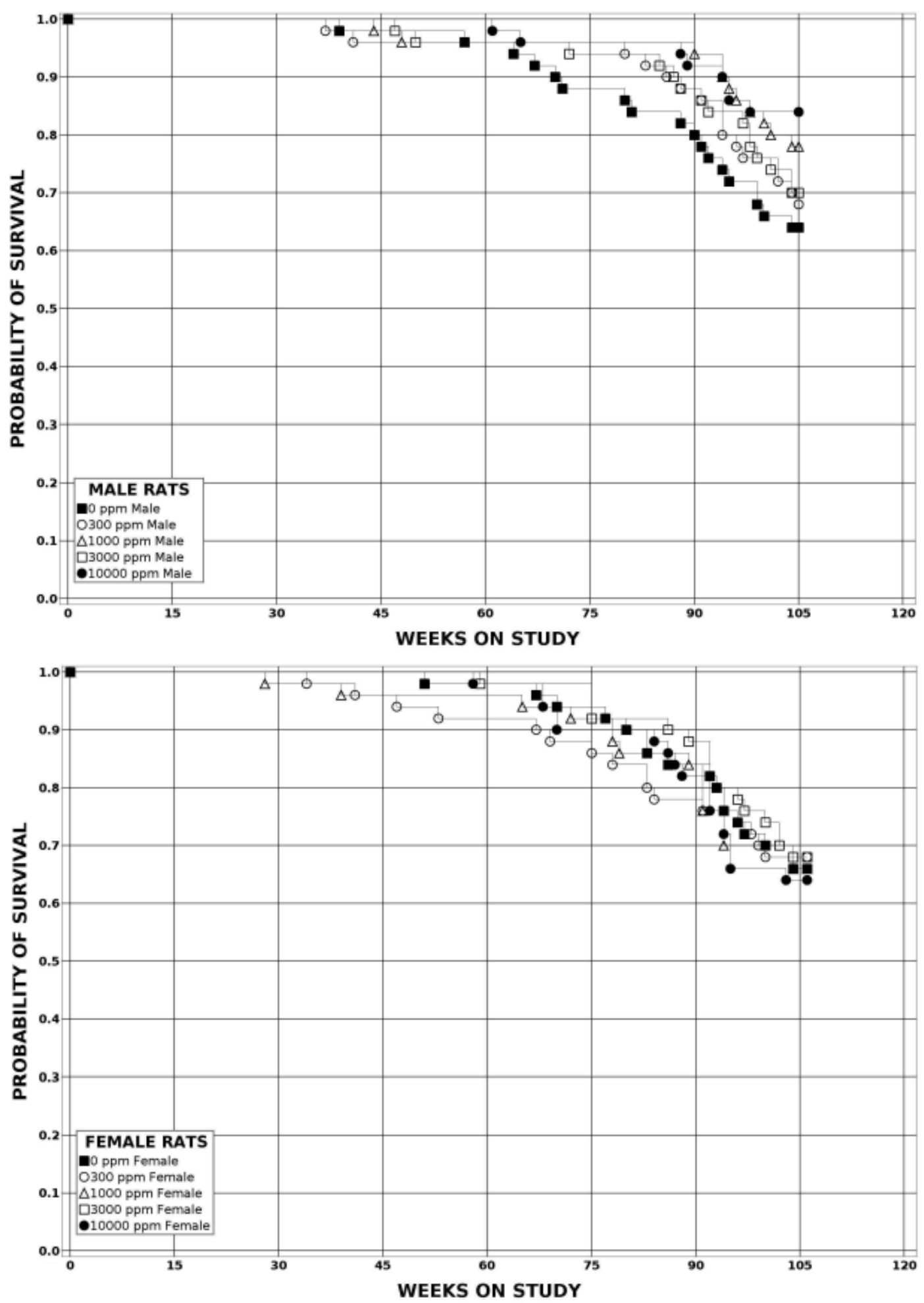

Figure 12. Kaplan-Meier Survival Curves for Rats Exposed to Di(2-ethylhexyl) Phthalate in the Postweaning-only Two-year Feed Study 
At study termination, group mean body weights for the 300, 1,000, and 3,000 ppm DEHP groups were within $6 \%$ of control animals in both male and female rats (Table 21, Table 22; Figure 13). Lower body weights were noted in males (approximately 16\%) and females (approximately $22 \%$ ) in the $10,000 \mathrm{ppm}$ groups at the end of study relative to control animals. These effects were attributed to reduced body weight gain relative to control animals, which occurred throughout the study.

Feed consumption by male and female rats in the 300, 1,000, 3,000, and 10,000 ppm DEHP groups was commensurate with that of the control group throughout the study with the exception of study week 1 , when feed consumption was approximately $21 \%$ lower in the $10,000 \mathrm{ppm}$ male and female groups (Table 23, Table 24). This finding might reflect an initial adjustment related to the palatability of feed containing high concentrations (1\%) of DEHP. Dietary concentrations of $300,1,000,3,000$, and 10,000 ppm resulted in average daily doses of approximately 17, 54, 170 , and $602 \mathrm{mg} / \mathrm{kg} /$ day for males and 17, 60, 177, and $646 \mathrm{mg} / \mathrm{kg} /$ day for females (Appendix H).

No exposure-related clinical findings were observed in any of the exposed groups (Appendix $\mathrm{H}$ ). 
Table 21. Summary of Survival and Mean Body Weights of Male Rats in the Postweaning-only Two-year Feed Study of Di(2-ethylhexyl) Phthalate

\begin{tabular}{|c|c|c|c|c|c|c|c|c|c|c|c|c|c|c|}
\hline \multirow[b]{2}{*}{$\begin{array}{l}\text { Study } \\
\text { Day }^{a}\end{array}$} & \multicolumn{2}{|c|}{0 ppm } & \multicolumn{3}{|c|}{300 ppm } & \multicolumn{3}{|c|}{$1,000 \mathrm{ppm}$} & \multicolumn{3}{|c|}{ 3,000 ppm } & \multicolumn{3}{|c|}{10,000 ppm } \\
\hline & $\begin{array}{l}\text { Av. } \\
\text { Wt. } \\
\text { (g) }\end{array}$ & $\begin{array}{c}\text { No. of } \\
\text { Survivors }\end{array}$ & $\begin{array}{l}\text { Av. } \\
\text { Wt. } \\
\text { (g) }\end{array}$ & $\begin{array}{c}\text { Wt. } \\
\text { (\% of } \\
\text { Controls) }\end{array}$ & $\begin{array}{c}\text { No. of } \\
\text { Survivors }\end{array}$ & $\begin{array}{l}\text { Av. } \\
\text { Wt. } \\
\text { (g) }\end{array}$ & $\begin{array}{c}\text { Wt. } \\
(\% \text { of } \\
\text { Controls) }\end{array}$ & $\begin{array}{c}\text { No. of } \\
\text { Survivors }\end{array}$ & $\begin{array}{l}\text { Av. } \\
\text { Wt. } \\
\text { (g) }\end{array}$ & $\begin{array}{c}\text { Wt. } \\
(\% \text { of } \\
\text { Controls })\end{array}$ & $\begin{array}{c}\text { No. of } \\
\text { Survivors }\end{array}$ & $\begin{array}{l}\text { Av. } \\
\text { Wt. } \\
\text { (g) }\end{array}$ & $\begin{array}{c}\text { Wt. } \\
(\% \text { of } \\
\text { Controls) }\end{array}$ & $\begin{array}{c}\text { No. of } \\
\text { Survivors }\end{array}$ \\
\hline 1 & 138.2 & 50 & 138.5 & 100.2 & 50 & 139.0 & 100.5 & 50 & 138.7 & 100.3 & 50 & 139.3 & 100.8 & 50 \\
\hline 8 & 182.5 & 50 & 182.4 & 100.0 & 50 & 184.1 & 100.9 & 50 & 182.3 & 99.9 & 50 & 174.1 & 95.4 & 50 \\
\hline 15 & 222.3 & 50 & 225.4 & 101.4 & 50 & 224.7 & 101.1 & 50 & 223.2 & 100.4 & 50 & 215.1 & 96.8 & 50 \\
\hline 22 & 253.2 & 50 & 256.9 & 101.5 & 50 & 254.6 & 100.6 & 50 & 253.4 & 100.1 & 50 & 243.0 & 96.0 & 50 \\
\hline 29 & 273.8 & 50 & 279.4 & 102.1 & 50 & 275.8 & 100.8 & 50 & 273.1 & 99.8 & 50 & 259.4 & 94.8 & 50 \\
\hline 36 & 290.0 & 50 & 296.6 & 102.3 & 50 & 293.0 & 101.0 & 50 & 288.7 & 99.6 & 50 & 271.2 & 93.5 & 50 \\
\hline 43 & 303.0 & 50 & 310.6 & 102.5 & 50 & 306.6 & 101.2 & 50 & 301.3 & 99.4 & 50 & 283.8 & 93.7 & 50 \\
\hline 50 & 314.9 & 50 & 318.7 & 101.2 & 50 & 316.7 & 100.6 & 50 & 311.8 & 99.0 & 50 & 294.0 & 93.4 & 50 \\
\hline 57 & 326.4 & 50 & 326.5 & 100.0 & 50 & 325.9 & 99.9 & 50 & 319.1 & 97.8 & 50 & 301.2 & 92.3 & 50 \\
\hline 64 & 337.6 & 50 & 337.4 & 99.9 & 50 & 336.8 & 99.8 & 50 & 328.6 & 97.3 & 50 & 309.5 & 91.7 & 50 \\
\hline 71 & 346.3 & 50 & 347.1 & 100.2 & 50 & 345.2 & 99.7 & 50 & 335.4 & 96.9 & 50 & 316.4 & 91.4 & 50 \\
\hline 78 & 357.7 & 50 & 354.7 & 99.1 & 50 & 354.3 & 99.0 & 50 & 343.8 & 96.1 & 50 & 323.0 & 90.3 & 50 \\
\hline 85 & 365.2 & 50 & 360.9 & 98.8 & 50 & 361.7 & 99.0 & 50 & 346.3 & 94.8 & 50 & 324.2 & 88.8 & 50 \\
\hline 92 & 372.1 & 50 & 365.2 & 98.2 & 50 & 366.3 & 98.5 & 50 & 351.0 & 94.3 & 50 & 329.4 & 88.5 & 50 \\
\hline 120 & 386.2 & 50 & 388.4 & 100.6 & 50 & 380.2 & 98.4 & 50 & 365.1 & 94.5 & 50 & 341.9 & 88.5 & 50 \\
\hline 148 & 404.6 & 50 & 409.5 & 101.2 & 50 & 398.5 & 98.5 & 50 & 385.6 & 95.3 & 50 & 361.1 & 89.3 & 50 \\
\hline 176 & 420.8 & 50 & 426.9 & 101.4 & 50 & 415.8 & 98.8 & 50 & 400.2 & 95.1 & 50 & 373.2 & 88.7 & 50 \\
\hline 204 & 435.4 & 50 & 435.6 & 100.0 & 50 & 428.4 & 98.4 & 50 & 409.0 & 93.9 & 50 & 375.8 & 86.3 & 50 \\
\hline 232 & 443.7 & 50 & 447.1 & 100.8 & 50 & 436.8 & 98.4 & 50 & 412.8 & 93.0 & 50 & 380.5 & 85.8 & 50 \\
\hline 260 & 455.5 & 50 & 463.2 & 101.7 & 49 & 451.1 & 99.0 & 50 & 430.5 & 94.5 & 50 & 393.2 & 86.3 & 50 \\
\hline 288 & 463.2 & 49 & 468.7 & 101.2 & 48 & 460.0 & 99.3 & 50 & 437.8 & 94.5 & 50 & 396.5 & 85.6 & 50 \\
\hline 316 & 475.3 & 49 & 477.8 & 100.5 & 48 & 467.8 & 98.4 & 49 & 444.2 & 93.5 & 50 & 401.4 & 84.4 & 50 \\
\hline 344 & 478.3 & 49 & 489.5 & 102.3 & 48 & 477.9 & 99.9 & 48 & 448.0 & 93.7 & 49 & 406.9 & 85.1 & 50 \\
\hline 372 & 481.2 & 49 & 490.2 & 101.9 & 48 & 481.6 & 100.1 & 48 & 457.6 & 95.1 & 48 & 412.7 & 85.8 & 50 \\
\hline 400 & 499.7 & 48 & 508.9 & 101.8 & 48 & 492.3 & 98.5 & 48 & 468.2 & 93.7 & 48 & 420.6 & 84.2 & 50 \\
\hline 428 & 508.5 & 48 & 510.8 & 100.5 & 48 & 501.2 & 98.6 & 48 & 472.1 & 92.8 & 48 & 422.7 & 83.1 & 49 \\
\hline 456 & 507.2 & 47 & 513.9 & 101.3 & 48 & 505.4 & 99.6 & 48 & 476.4 & 93.9 & 48 & 424.2 & 83.6 & 48 \\
\hline 484 & 515.8 & 46 & 521.1 & 101.0 & 48 & 514.3 & 99.7 & 48 & 481.2 & 93.3 & 48 & 430.8 & 83.5 & 48 \\
\hline 512 & 515.5 & 44 & 522.5 & 101.4 & 48 & 511.6 & 99.2 & 48 & 481.9 & 93.5 & 47 & 432.7 & 83.9 & 48 \\
\hline 540 & 522.6 & 44 & 519.9 & 99.5 & 48 & 516.3 & 98.8 & 48 & 489.0 & 93.6 & 47 & 435.8 & 83.4 & 48 \\
\hline 568 & 525.8 & 42 & 528.0 & 100.4 & 47 & 524.2 & 99.7 & 48 & 490.8 & 93.4 & 47 & 436.0 & 82.9 & 48 \\
\hline 596 & 523.6 & 42 & 527.8 & 100.8 & 46 & 524.2 & 100.1 & 48 & 493.7 & 94.3 & 46 & 436.3 & 83.3 & 48 \\
\hline 624 & 517.5 & 41 & 536.6 & 103.7 & 44 & 520.8 & 100.6 & 47 & 496.8 & 96.0 & 44 & 435.0 & 84.1 & 46 \\
\hline 652 & 525.7 & 38 & 530.6 & 100.9 & 43 & 520.5 & 99.0 & 47 & 501.6 & 95.4 & 42 & 428.5 & 81.5 & 46 \\
\hline 680 & 515.6 & 36 & 530.7 & 102.9 & 38 & 522.5 & 101.3 & 43 & 501.8 & 97.3 & 40 & 427.4 & 82.9 & 43 \\
\hline 708 & 512.4 & 33 & 537.6 & 104.9 & 38 & 524.9 & 102.4 & 40 & 501.0 & 97.8 & 37 & 430.2 & 83.9 & 42 \\
\hline EOS & 505.9 & 32 & 524.8 & 103.7 & 33 & 520.6 & 102.9 & 39 & 500.9 & 99.0 & 35 & 426.9 & 84.4 & 42 \\
\hline
\end{tabular}

EOS $=$ end of study.

aStudy day 1 is the day animals were placed on study. 
Table 22. Summary of Survival and Mean Body Weights of Female Rats in the Postweaning-only Two-year Feed Study of Di(2-ethylhexyl) Phthalate

\begin{tabular}{|c|c|c|c|c|c|c|c|c|c|c|c|c|c|c|}
\hline \multirow[b]{2}{*}{$\begin{array}{l}\text { Study } \\
\text { Day }^{\mathrm{a}}\end{array}$} & \multicolumn{2}{|c|}{0 ppm } & \multicolumn{3}{|c|}{300 ppm } & \multicolumn{3}{|c|}{1,000 ppm } & \multicolumn{3}{|c|}{$3,000 \mathrm{ppm}$} & \multicolumn{3}{|c|}{10,000 ppm } \\
\hline & $\begin{array}{l}\text { Av. } \\
\text { Wt. } \\
\text { (g) }\end{array}$ & $\begin{array}{c}\text { No. of } \\
\text { Survivors }\end{array}$ & $\begin{array}{l}\text { Av. } \\
\text { Wt. } \\
\text { (g) }\end{array}$ & $\begin{array}{c}\text { Wt. } \\
(\% \text { of } \\
\text { Controls })\end{array}$ & $\begin{array}{c}\text { No. of } \\
\text { Survivors }\end{array}$ & $\begin{array}{l}\text { Av. } \\
\text { Wt. } \\
\text { (g) }\end{array}$ & $\begin{array}{c}\text { Wt. } \\
(\% \text { of } \\
\text { Controls })\end{array}$ & $\begin{array}{c}\text { No. of } \\
\text { Survivors }\end{array}$ & $\begin{array}{l}\text { Av. } \\
\text { Wt. } \\
\text { (g) }\end{array}$ & $\begin{array}{c}\text { Wt. } \\
(\% \text { of } \\
\text { Controls })\end{array}$ & $\begin{array}{c}\text { No. of } \\
\text { Survivors }\end{array}$ & $\begin{array}{l}\text { Av. } \\
\text { Wt. } \\
\text { (g) }\end{array}$ & $\begin{array}{c}\text { Wt. } \\
(\% \text { of } \\
\text { Controls })\end{array}$ & $\begin{array}{c}\text { No. of } \\
\text { Survivors }\end{array}$ \\
\hline 1 & 118.4 & 50 & 121.2 & 102.4 & 50 & 120.2 & 101.6 & 50 & 121.2 & 102.4 & 50 & 119.7 & 101.1 & 50 \\
\hline 8 & 141.7 & 50 & 141.0 & 99.5 & 50 & 141.2 & 99.6 & 50 & 144.2 & 101.8 & 50 & 139.3 & 98.3 & 50 \\
\hline 15 & 158.6 & 50 & 161.4 & 101.8 & 50 & 158.4 & 99.9 & 50 & 162.4 & 102.4 & 50 & 156.8 & 98.9 & 50 \\
\hline 22 & 172.5 & 50 & 177.4 & 102.9 & 50 & 174.7 & 101.3 & 50 & 179.9 & 104.3 & 50 & 170.6 & 98.9 & 50 \\
\hline 29 & 185.0 & 50 & 193.2 & 104.4 & 50 & 185.5 & 100.2 & 50 & 189.4 & 102.3 & 50 & 178.3 & 96.3 & 50 \\
\hline 36 & 193.7 & 50 & 200.9 & 103.7 & 50 & 194.1 & 100.2 & 50 & 200.0 & 103.2 & 50 & 185.8 & 95.9 & 50 \\
\hline 43 & 200.1 & 50 & 207.7 & 103.8 & 50 & 203.0 & 101.5 & 50 & 206.8 & 103.4 & 50 & 191.9 & 95.9 & 50 \\
\hline 50 & 205.8 & 50 & 212.4 & 103.2 & 50 & 210.6 & 102.3 & 50 & 210.9 & 102.5 & 50 & 196.0 & 95.3 & 50 \\
\hline 57 & 211.4 & 50 & 219.0 & 103.6 & 50 & 217.2 & 102.7 & 50 & 216.0 & 102.2 & 50 & 201.7 & 95.4 & 50 \\
\hline 64 & 216.4 & 50 & 221.1 & 102.2 & 50 & 223.0 & 103.0 & 50 & 220.8 & 102.1 & 50 & 205.0 & 94.7 & 50 \\
\hline 71 & 220.5 & 50 & 226.4 & 102.7 & 50 & 225.4 & 102.2 & 50 & 225.1 & 102.1 & 50 & 208.3 & 94.5 & 50 \\
\hline 78 & 224.9 & 50 & 228.6 & 101.7 & 50 & 230.8 & 102.7 & 50 & 229.7 & 102.1 & 50 & 213.0 & 94.7 & 50 \\
\hline 85 & 228.8 & 50 & 231.0 & 100.9 & 50 & 231.8 & 101.3 & 50 & 233.0 & 101.8 & 50 & 215.0 & 94.0 & 50 \\
\hline 92 & 233.4 & 50 & 232.7 & 99.7 & 50 & 234.4 & 100.4 & 50 & 233.4 & 100.0 & 50 & 216.8 & 92.9 & 50 \\
\hline 120 & 241.1 & 50 & 248.2 & 102.9 & 50 & 240.8 & 99.9 & 50 & 242.7 & 100.6 & 50 & 221.6 & 91.9 & 50 \\
\hline 148 & 248.1 & 50 & 255.7 & 103.1 & 50 & 250.7 & 101.1 & 50 & 251.2 & 101.3 & 50 & 229.3 & 92.4 & 50 \\
\hline 176 & 253.9 & 50 & 267.2 & 105.2 & 50 & 259.7 & 102.3 & 50 & 258.6 & 101.8 & 50 & 234.5 & 92.4 & 50 \\
\hline 204 & 258.3 & 50 & 266.6 & 103.2 & 50 & 261.3 & 101.1 & 49 & 260.8 & 100.9 & 50 & 235.4 & 91.1 & 50 \\
\hline 232 & 264.1 & 50 & 276.6 & 104.8 & 50 & 262.1 & 99.3 & 49 & 266.2 & 100.8 & 50 & 236.3 & 89.5 & 50 \\
\hline 260 & 265.8 & 50 & 280.1 & 105.4 & 49 & 270.9 & 101.9 & 49 & 269.5 & 101.4 & 50 & 240.7 & 90.5 & 50 \\
\hline 288 & 272.7 & 50 & 285.2 & 104.6 & 48 & 271.4 & 99.5 & 48 & 273.2 & 100.2 & 50 & 241.5 & 88.6 & 50 \\
\hline 316 & 274.2 & 50 & 289.6 & 105.6 & 48 & 274.0 & 99.9 & 48 & 273.8 & 99.8 & 50 & 241.9 & 88.2 & 50 \\
\hline 344 & 279.5 & 50 & 291.4 & 104.3 & 47 & 277.8 & 99.4 & 48 & 278.6 & 99.7 & 50 & 242.5 & 86.8 & 50 \\
\hline 372 & 283.3 & 49 & 295.7 & 104.4 & 46 & 282.1 & 99.6 & 48 & 281.3 & 99.3 & 50 & 242.8 & 85.7 & 50 \\
\hline 400 & 290.1 & 49 & 301.8 & 104.0 & 46 & 286.3 & 98.7 & 48 & 284.9 & 98.2 & 50 & 244.4 & 84.2 & 50 \\
\hline 428 & 295.3 & 49 & 303.2 & 102.7 & 46 & 293.0 & 99.2 & 48 & 291.1 & 98.6 & 49 & 247.7 & 83.9 & 49 \\
\hline 456 & 300.7 & 49 & 309.8 & 103.0 & 46 & 296.3 & 98.6 & 47 & 293.5 & 97.6 & 49 & 249.0 & 82.8 & 49 \\
\hline 484 & 304.6 & 48 & 305.8 & 100.4 & 44 & 302.5 & 99.3 & 47 & 297.4 & 97.6 & 49 & 252.0 & 82.7 & 47 \\
\hline 512 & 308.3 & 47 & 313.1 & 101.6 & 44 & 307.0 & 99.6 & 46 & 302.8 & 98.2 & 49 & 251.7 & 81.6 & 45 \\
\hline 540 & 308.6 & 46 & 314.6 & 101.9 & 43 & 316.8 & 102.6 & 46 & 300.2 & 97.3 & 46 & 251.9 & 81.6 & 45 \\
\hline 568 & 311.3 & 45 & 324.0 & 104.1 & 42 & 313.8 & 100.8 & 43 & 299.2 & 96.1 & 46 & 253.2 & 81.3 & 45 \\
\hline 596 & 315.8 & 43 & 319.2 & 101.1 & 39 & 320.9 & 101.6 & 43 & 303.2 & 96.0 & 46 & 251.5 & 79.6 & 43 \\
\hline 624 & 321.7 & 42 & 322.6 & 100.3 & 39 & 327.3 & 101.7 & 42 & 304.4 & 94.6 & 44 & 251.4 & 78.2 & 41 \\
\hline 652 & 325.1 & 40 & 325.6 & 100.1 & 38 & 327.3 & 100.7 & 36 & 307.6 & 94.6 & 40 & 247.4 & 76.1 & 37 \\
\hline 680 & 324.4 & 36 & 328.8 & 101.4 & 36 & 322.6 & 99.5 & 35 & 311.3 & 96.0 & 38 & 247.3 & 76.2 & 33 \\
\hline 708 & 329.5 & 35 & 328.9 & 99.8 & 34 & 334.3 & 101.4 & 35 & 312.0 & 94.7 & 36 & 247.7 & 75.2 & 33 \\
\hline EOS & 322.5 & 33 & 336.3 & 104.3 & 34 & 341.5 & 105.9 & 31 & 312.5 & 96.9 & 34 & 252.0 & 78.1 & 32 \\
\hline
\end{tabular}

$\mathrm{EOS}=$ end of study.

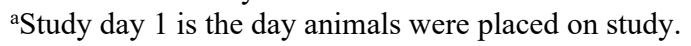



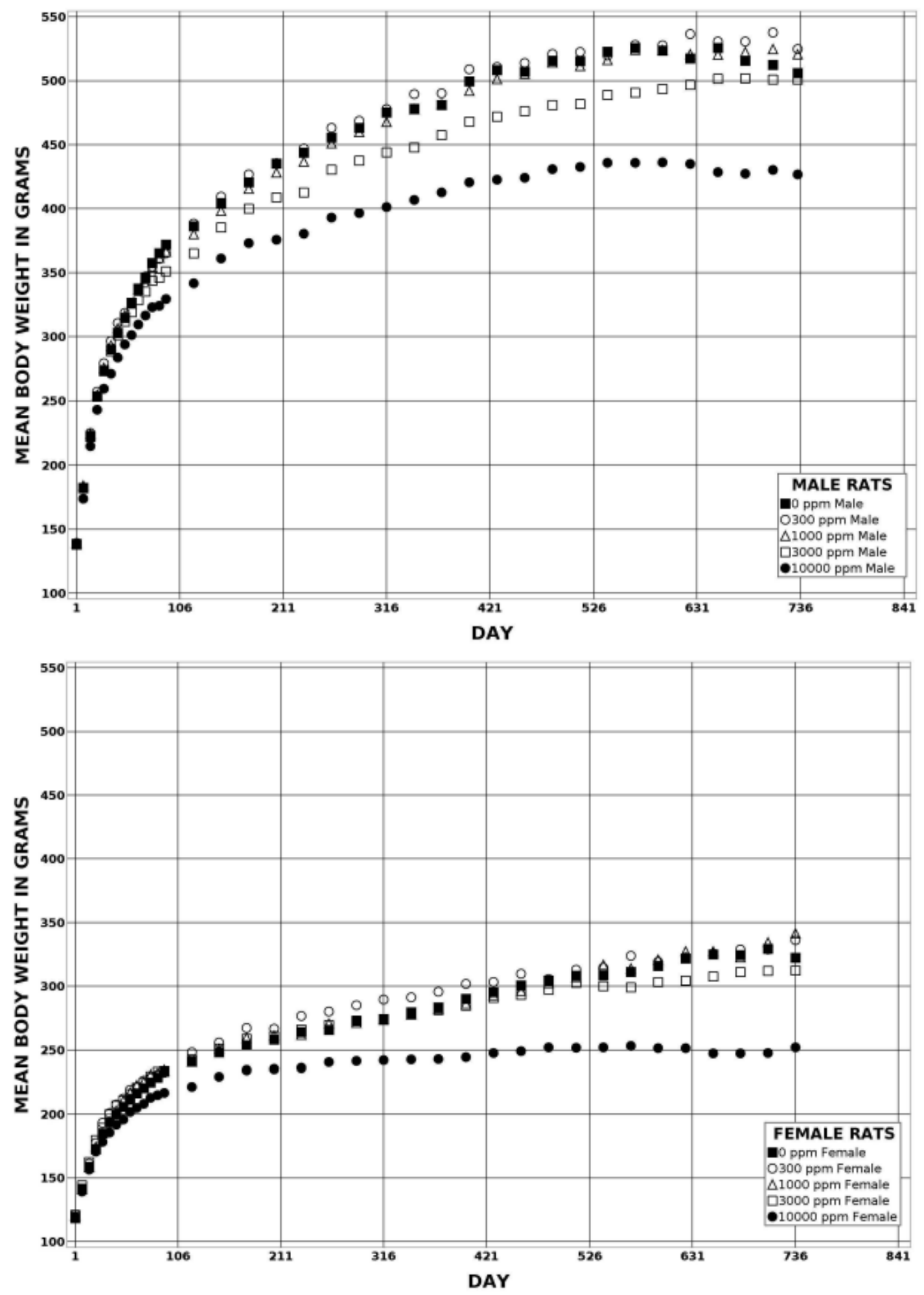

Figure 13. Growth Curves for Rats Exposed to Di(2-ethylhexyl) Phthalate in the Postweaning-only Two-year Feed Study 
Table 23. Summary of Feed and Di(2-ethylhexyl) Phthalate Consumption of Male Rats in the Postweaning-only Two-year Feed Study

\begin{tabular}{cccccccccc}
\hline \multirow{2}{*}{ Week } & $\mathbf{0 ~} \mathbf{~ p p m}$ & \multicolumn{2}{c}{$\mathbf{3 0 0} \mathbf{~ p p m}$} & \multicolumn{2}{c}{$\mathbf{1 , 0 0 0} \mathbf{~ p p m}$} & \multicolumn{2}{c}{$\mathbf{3 , 0 0 0} \mathbf{~ p p m}$} & \multicolumn{2}{c}{$\mathbf{1 0 , 0 0 0} \mathbf{~ p p m}$} \\
\cline { 2 - 9 } & $\begin{array}{c}\text { Feed } \\
(\mathbf{g} / \mathbf{d a y})^{\mathbf{2}}\end{array}$ & $\begin{array}{c}\text { Feed } \\
(\mathbf{g} / \mathbf{d a y})\end{array}$ & $\begin{array}{c}\text { Dose } \\
(\mathbf{m g} / \mathbf{k g} / \mathbf{d a y})^{\mathbf{b}}\end{array}$ & $\begin{array}{c}\text { Feed } \\
(\mathbf{g} / \mathbf{d a y})\end{array}$ & $\begin{array}{c}\text { Dose } \\
(\mathbf{m g} / \mathbf{k g} / \mathbf{d a y})\end{array}$ & $\begin{array}{c}\text { Feed } \\
(\mathbf{g} / \mathbf{d a y})\end{array}$ & $\begin{array}{c}\text { Dose } \\
(\mathbf{m g} / \mathbf{k g} / \mathbf{d a y})\end{array}$ & $\begin{array}{c}\text { Feed } \\
(\mathbf{g} / \mathbf{d a y})\end{array}$ & $\begin{array}{c}\text { Dose } \\
(\mathbf{m g} / \mathbf{k g} / \mathbf{d a y})\end{array}$ \\
\hline 1 & 20.2 & 20.4 & 44.2 & 20.4 & 146.8 & 19.8 & 428.2 & 15.9 & $1,141.5$ \\
13 & 21.6 & 20.2 & 16.8 & 20.2 & 55.9 & 20.5 & 177.6 & 19.3 & 595.3 \\
54 & 27.4 & 26.3 & 16.1 & 26.7 & 55.4 & 26.3 & 172.4 & 24.0 & 581.5 \\
102 & 25.1 & 26.4 & 14.7 & 25.1 & 47.8 & 23.6 & 141.3 & 24.5 & 569.5 \\
\hline
\end{tabular}

${ }^{\mathrm{a}}$ Grams of feed consumed per animal per day.

${ }^{\mathrm{b}}$ Milligrams of di(2-ethylhexyl) phthalate consumed/kilogram body weight/day.

Table 24. Summary of Feed and Di(2-ethylhexyl) Phthalate Consumption of Female Rats in the Postweaning-only Two-year Feed Study

\begin{tabular}{ccccccccccc}
\hline \multirow{2}{*}{ Week } & $\mathbf{0 ~ p p m}$ & \multicolumn{2}{c}{$\mathbf{3 0 0} \mathbf{~ p p m}$} & \multicolumn{2}{c}{$\mathbf{1 , 0 0 0} \mathbf{~ p p m}$} & \multicolumn{2}{c}{$\mathbf{3 , 0 0 0} \mathbf{~ p p m}$} & \multicolumn{2}{c}{$\mathbf{1 0 , 0 0 0} \mathbf{~ p p m}$} \\
\cline { 2 - 9 } & $\begin{array}{c}\text { Feed } \\
(\mathbf{g} / \mathbf{d a y})^{\mathbf{a}}\end{array}$ & $\begin{array}{c}\text { Feed } \\
(\mathbf{g} / \mathbf{d a y})\end{array}$ & $\begin{array}{c}\text { Dose } \\
(\mathbf{m g} / \mathbf{k g} / \mathbf{d a y})^{\mathbf{b}}\end{array}$ & $\begin{array}{c}\text { Feed } \\
(\mathbf{g} / \mathbf{d a y})\end{array}$ & $\begin{array}{c}\text { Dose } \\
(\mathbf{m g} / \mathbf{k g} / \mathbf{d a y})\end{array}$ & $\begin{array}{c}\text { Feed } \\
(\mathbf{g} / \mathbf{d a y})\end{array}$ & $\begin{array}{c}\text { Dose } \\
(\mathbf{m g} / \mathbf{k g} / \mathbf{d a y})\end{array}$ & $\begin{array}{c}\text { Feed } \\
(\mathbf{g} / \mathbf{d a y})\end{array}$ & $\begin{array}{c}\text { Dose } \\
(\mathbf{m g} / \mathbf{k g} / \mathbf{d a y})\end{array}$ \\
\hline 1 & 14.5 & 14.5 & 35.9 & 14.2 & 118.1 & 14.6 & 361.5 & 11.5 & 960.6 \\
13 & 14.0 & 13.2 & 17.1 & 14.6 & 63.0 & 14.2 & 182.8 & 13.6 & 632.7 \\
54 & 15.0 & 17.2 & 17.4 & 16.5 & 58.5 & 16.3 & 173.9 & 15.1 & 621.9 \\
102 & 18.1 & 18.3 & 16.7 & 18.8 & 56.2 & 18.6 & 175.5 & 16.9 & 682.2 \\
\hline
\end{tabular}

${ }^{\mathrm{a}}$ Grams of feed consumed per animal per day.

${ }^{b}$ Milligrams of di(2-ethylhexyl) phthalate consumed/kilogram body weight/day.

\section{Pathology}

This section describes the statistically significant or biologically noteworthy changes in the incidences of neoplasms and/or nonneoplastic lesions of the liver, pancreas, male and female reproductive organs, heart, bone marrow, and pituitary gland. Summaries of the incidences of neoplasms and nonneoplastic lesions, individual animal neoplasm diagnoses, and statistical analyses of primary neoplasms that occurred with an incidence of at least $5 \%$ in at least one animal group are presented as supplemental data in Appendix $\mathrm{H}$.

Liver: There were significant increases in the incidences of hepatocellular adenomas (10,000 ppm males and females) and carcinomas (10,000 ppm males) relative to that of control animals and a positive trend in females for hepatocellular carcinomas with increasing exposure concentration (Table 25). The incidence of adenoma or carcinoma (combined) was significantly increased in the 10,000 ppm male and female groups relative to the respective control groups. Hepatocellular adenomas were characterized by regions that were sharply demarcated from surrounding liver parenchyma, nodular, and compressing adjacent normal hepatocytes, with loss of normal lobular architecture and an irregular growth pattern. The liver plates typically impinged obliquely on the surrounding liver parenchyma. The hepatocytes within an adenoma generally varied in size. Hepatocellular carcinomas were characterized by one or more of the following features: local infiltrating growth and/or distinct lack of demarcation with the adjacent tissue, the presence of trabeculae composed of multiple layers of hepatocytes, cellular 
pleomorphism, loss of normal lobular architecture, regions of hemorrhage and/or necrosis, and increased mitotic figures.

There were significant increases in the incidences of many nonneoplastic liver lesions in DEHPexposed groups relative to the control groups (Table 25). The incidence of hepatocellular cytoplasmic alteration was significantly increased in 3,000 and 10,000 ppm males and in 1,000, 3,000 , and 10,000 ppm females relative to that of the respective control animals. There were significant increases in the incidence of hepatocellular hypertrophy in the 10,000 ppm males and in the $1,000,3,000$, and 10,000 ppm females. Significant increases in the incidence of liver pigment were observed in the 1,000,3,000, and 10,000 ppm males and females. There were significant increases in the incidence of liver necrosis in the 3,000 and 10,000 ppm males. There was a significant increase in the incidence of hepatocellular eosinophilic foci in the $10,000 \mathrm{ppm}$ males, and a positive trend in the incidence of hepatocellular clear cell foci in exposed males with increasing exposure concentration.

Hepatocellular cytoplasmic alteration was characterized by hepatocytes that were expanded with eosinophilic granular cytoplasm (see Figure 5 as an example). A four-grade severity scale was used based on degree of tissue affected in the section of liver that was evaluated histologically: minimal (grade 1), up to $25 \%$ of hepatocyte involvement; mild (grade 2 ), $26 \%$ to $50 \%$ of hepatocyte involvement; moderate (grade 3), $51 \%$ to $75 \%$ of hepatocyte involvement; and marked (grade 4 ) at least $76 \%$ of hepatocyte involvement. Hepatocellular hypertrophy often occurred in conjunction with cytoplasmic alteration and/or pigment. Hypertrophy was characterized by enlargement of the hepatocytes. In lesser affected animals, hypertrophy was confined to centrilobular regions, but in more severely affected animals, hypertrophy extended into the midzonal and periportal areas. A four-grade severity scale was used: minimal (grade 1), up to $10 \%$ of hepatocyte involvement; mild (grade 2), $11 \%$ to $25 \%$ of hepatocyte involvement; moderate (grade 3 ), $26 \%$ to $50 \%$ of hepatocyte involvement; and severe (grade 4 ), $\geq 51 \%$ of hepatic involvement. Hypertrophy was generally centrilobular and often involved only a few cells per lobule. Although hypertrophy was only occasionally observed in males (at the 3,000 and 10,000 ppm concentrations), in females, its incidence (but not severity) increased significantly with exposure concentrations starting at 1,000 ppm.

Pigment was characterized by a pale gold-colored pigment within the hepatocellular cytoplasm (see Figure 5 as an example). A four-grade severity scale was used: minimal (grade 1), up to $30 \%$ of hepatocytes contained pigment; mild (grade 2), 31\% to 50\% of hepatocytes contained pigment; moderate (grade 3), $\geq 51 \%$ of hepatocytes contained pigment; and marked (grade 4), $>51 \%$ of hepatocytes contained pigment, and the pigment was very dense. Hepatocellular necrosis was characterized by multiple adjacent hepatocytes that were swollen with increased eosinophilia, karyorrhectic nuclear debris, with or without accompanying inflammatory cells. Necrosis was scored using a four-grade severity scale: minimal (grade 1), up to three focal areas of necrosis present; mild (grade 2), necrosis in up to $25 \%$ of the liver; moderate (grade 3 ), necrosis in $26 \%$ to $60 \%$ of the liver; and severe (grade 4 ), necrosis in $\geq 61 \%$ of the liver.

Hepatocellular foci were diagnosed when there was an alteration in the arrangement of hepatocytes involving at least six cells, with a discrete lesion margin, where attenuated hepatocytes at the lesion margin (compression) involved $<70 \%$ of the lesion circumference. Lobular architecture was preserved in the absence of cellular atypia, and the hepatocytes within the eosinophilic foci were more eosinophilic than those within the surrounding parenchyma. 
Clear cell foci were characterized by circular or ovoid regions composed of normal-sized or enlarged cells with distinct cytoplasmic clear spaces compared with the surrounding parenchyma. The nuclei were often small and dense, prominent, and centrally located, occasionally exhibiting increased volume. The distinction between large foci (usually eosinophilic) and hepatocellular adenomas was based on retention of normal lobular architecture in the foci, greater size of hepatocellular adenomas (usually measuring at least $3 \mathrm{~mm}$ ), and presence of compression or bulging of the adenoma from the liver surface along $>70 \%$ of the lesion circumference.

Table 25. Incidences of Neoplastic and Nonneoplastic Lesions of the Liver in Male and Female Rats in the Postweaning-only Two-year Feed Study of Di(2-ethylhexyl) Phthalate

\begin{tabular}{|c|c|c|c|c|c|}
\hline & 0 ppm & 300 ppm & 1,000 ppm & 3,000 ppm & $10,000 \mathrm{ppm}$ \\
\hline \multicolumn{6}{|l|}{ Male } \\
\hline $\mathbf{n}^{\mathbf{a}}$ & 50 & 50 & 50 & 50 & 50 \\
\hline $\begin{array}{l}\text { Hepatocyte, Cytoplasmic } \\
\text { Alteration }\end{array}$ & $0 * *$ & $1(2.0)^{\mathrm{c}}$ & 0 & $38 * *(1.3)$ & $49 * *(3.6)$ \\
\hline Hepatocyte, Hypertrophy & $0 * *$ & 0 & 0 & $2(1.0)$ & $6 *(1.2)$ \\
\hline Pigment & $0 * *$ & 0 & $7 *(1.0)$ & $45^{* *}(1.8)$ & $50 * *(2.5)$ \\
\hline Necrosis & $0^{* *}$ & $2(1.5)$ & $4(1.0)$ & $7 *(1.3)$ & $8 * *(1.3)$ \\
\hline Eosinophilic Focus & $1 * *$ & 0 & 4 & 2 & $24 * *$ \\
\hline Clear Cell Focus & $29 *$ & 31 & 33 & 35 & 39 \\
\hline \multicolumn{6}{|l|}{ Hepatocellular Adenoma $^{\mathrm{d}}$} \\
\hline Overall rate ${ }^{e}$ & $0 / 50(0 \%)$ & $2 / 50(4 \%)$ & $0 / 50(0 \%)$ & $1 / 50(2 \%)$ & $6 / 50(12 \%)$ \\
\hline Adjusted rate ${ }^{f}$ & $0 \%$ & $4.5 \%$ & $0 \%$ & $2.2 \%$ & $12.9 \%$ \\
\hline Poly-3 test ${ }^{\mathrm{g}}$ & $\mathrm{p}<0.001$ & $\mathrm{p}=0.251$ & (e) & $\mathrm{p}=0.514$ & $\mathrm{p}=0.022$ \\
\hline \multicolumn{6}{|l|}{ Hepatocellular Carcinoma ${ }^{\mathrm{h}}$} \\
\hline Overall rate & $0 / 50(0 \%)$ & $0 / 50(0 \%)$ & $0 / 50(0 \%)$ & $0 / 50(0 \%)$ & $6 / 50(12 \%)$ \\
\hline Adjusted rate & $0 \%$ & $0 \%$ & $0 \%$ & $0 \%$ & $12.8 \%$ \\
\hline Poly-3 test & $\mathrm{p}<0.001$ & (e) & (e) & (e) & $\mathrm{p}=0.022$ \\
\hline \multicolumn{6}{|c|}{ Hepatocellular Adenoma or Carcinoma (Combined) } \\
\hline Overall rate & $0 / 50(0 \%)$ & $2 / 50(4 \%)$ & $0 / 50(0 \%)$ & $1 / 50(2 \%)$ & $12 / 50(24 \%)$ \\
\hline Adjusted rate & $0 \%$ & $4.5 \%$ & $0 \%$ & $2.2 \%$ & $25.6 \%$ \\
\hline Poly-3 test & $\mathrm{p}<0.001$ & $\mathrm{p}=0.251$ & (e) & $\mathrm{p}=0.514$ & $\mathrm{p}<0.001$ \\
\hline \multicolumn{6}{|l|}{ Female } \\
\hline $\mathbf{n}$ & 50 & 50 & 50 & 50 & 49 \\
\hline $\begin{array}{l}\text { Hepatocyte, Cytoplasmic } \\
\text { Alteration }\end{array}$ & $0^{* *}$ & $2(1.0)$ & $15^{* *}(1.1)$ & $38 * *(1.3)$ & $45^{* *}(2.8)$ \\
\hline Hepatocyte, Hypertrophy & $0^{* *}$ & 0 & $6 *(1.2)$ & $14 * *(1.0)$ & $28 * *(1.3)$ \\
\hline Pigment & $3 * *(1.0)$ & 0 & $18^{* *}(1.1)$ & $30 * *(1.3)$ & $48 * *(2.5)$ \\
\hline Necrosis & $2(1.0)$ & $5(1.8)$ & $4(1.5)$ & $2(2.5)$ & $4(2.0)$ \\
\hline
\end{tabular}




\begin{tabular}{|c|c|c|c|c|c|}
\hline & O ppm & 300 ppm & $1,000 \mathrm{ppm}$ & $3,000 \mathrm{ppm}$ & $10,000 \mathrm{ppm}$ \\
\hline Eosinophilic Focus & 7 & 6 & 6 & 3 & 7 \\
\hline Clear Cell Focus & 8 & 10 & 14 & 7 & 5 \\
\hline \multicolumn{6}{|c|}{ Hepatocellular Adenoma ${ }^{j}$} \\
\hline Overall rate & $0 / 50(0 \%)$ & $0 / 50(0 \%)$ & $1 / 50(2 \%)$ & $1 / 50(2 \%)$ & $13 / 49(27 \%)$ \\
\hline Adjusted rate & $0 \%$ & $0 \%$ & $2.4 \%$ & $2.3 \%$ & $31.3 \%$ \\
\hline Poly-3 test & $\mathrm{p}<0.001$ & (e) & $\mathrm{p}=0.495$ & $\mathrm{p}=0.505$ & $\mathrm{p}<0.001$ \\
\hline \multicolumn{6}{|c|}{ Hepatocellular Carcinoma ${ }^{\mathrm{k}}$} \\
\hline Overall rate & $0 / 50(0 \%)$ & $0 / 50(0 \%)$ & $0 / 50(0 \%)$ & $0 / 50(0 \%)$ & $2 / 49(4 \%)$ \\
\hline Adjusted rate & $0 \%$ & $0 \%$ & $0 \%$ & $0 \%$ & $4.9 \%$ \\
\hline Poly-3 test & $\mathrm{p}=0.018$ & (e) & (e) & (e) & $\mathrm{p}=0.226$ \\
\hline \multicolumn{6}{|c|}{ Hepatocellular Adenoma or Carcinoma (Combined) $)^{1}$} \\
\hline Overall rate & $0 / 50(0 \%)$ & $0 / 50(0 \%)$ & $1 / 50(2 \%)$ & $1 / 50(2 \%)$ & $14 / 49(29 \%)$ \\
\hline Adjusted rate & $0 \%$ & $0 \%$ & $2.4 \%$ & $2.3 \%$ & $33.7 \%$ \\
\hline Poly-3 test & $\mathrm{p}<0.001$ & (e) & $\mathrm{p}=0.495$ & $\mathrm{p}=0.505$ & $\mathrm{p}<0.001$ \\
\hline
\end{tabular}

Statistical significance for an exposure group indicates a significant pairwise test compared to the vehicle control group.

Statistical significance for the vehicle control group indicates a significant trend test.

*Statistically significant at $\mathrm{p} \leq 0.05$ by the Poly- 3 test; ** $\mathrm{p} \leq 0.01$.

(e) = value of statistic could not be computed.

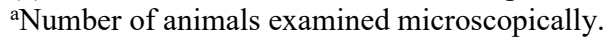

${ }^{b}$ Number of animals with lesion.

${ }^{\mathrm{c}}$ Average severity grade of lesions in affected animals: $1=$ minimal, $2=$ mild, $3=$ moderate, $4=$ marked.

${ }^{\mathrm{d}}$ Historical control incidence for all routes of 2-year studies (mean \pm standard deviation): $2 / 489(0.44 \% \pm 0.88 \%)$;

range: $0 \%$ to $2 \%$.

${ }^{\text {e}}$ Number of animals with neoplasm per number of animals necropsied.

fPoly-3 estimated neoplasm incidence after adjustment for intercurrent mortality.

${ }^{g}$ Beneath the control incidence is the $p$ value associated with the trend test. Beneath the exposed group incidence are the $p$ values corresponding to pairwise comparisons between the vehicle control group and that exposed group. The Poly-3 test accounts for differential mortality in animals that do not reach study termination. A negative trend or a lower incidence in an exposure group is indicated by $\mathrm{N}$.

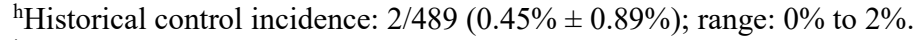

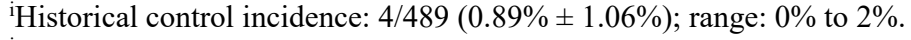

${ }^{j}$ Historical control incidence: $15 / 489(2.65 \% \pm 2.59 \%)$; range: $0 \%$ to $8 \%$.

${ }^{k}$ Historical control incidence: $1 / 489(0.22 \% \pm 0.67 \%)$; range: $0 \%$ to $2 \%$.

${ }^{l}$ Historical control incidence: $16 / 489(2.87 \% \pm 2.8 \%)$; range: $0 \%$ to $8 \%$.

Pancreas: In male rats, there were significant increases in the incidences of acinar adenoma and acinar adenoma or carcinoma (combined) in the 3,000 and 10,000 ppm groups relative to the control group. There was a significant increase in the incidence of acinar carcinoma in the 10,000 ppm male group (Table 26). In females, there was a positive trend for pancreas acinar adenoma or carcinoma (combined). Pancreatic acinar adenomas were distinct nodular masses that were not contiguous with the adjacent parenchyma, which were $>3 \mathrm{~mm}$ in diameter, and that compressed the adjacent tissue; pleomorphism or atypia was rarely present. Pancreatic acinar carcinomas were typically larger than adenomas and frequently exhibited cellular pleomorphism and atypia; invasion or metastasis was pathognomonic. Scirrhous reactions were occasionally present, characterized by dense fibrous or connective tissue. 
There were significant increases in the incidences of pancreatic acinus hyperplasia in male rats in the 3,000 and 10,000 ppm groups relative to the control group. In females, there was a significant increase in the incidence of pancreatic acinus hyperplasia in the 10,000 ppm group. Pancreatic acinus hyperplasia was characterized by circumscribed areas of enlarged acini that were $<3 \mathrm{~mm}$ in diameter and that were contiguous with the adjacent parenchyma. A four-grade severity scale was used: minimal (grade 1), no more than one lobule was affected, and the lesion was smaller than $1 \mathrm{~mm}$; mild (grade 2), lesion was 1 to $2 \mathrm{~mm}$; moderate (grade 3), lesion was 2 to $3 \mathrm{~mm}$; and marked (grade 4), lesion was $3 \mathrm{~mm}$ but lacked features of an adenoma, such as compression. Severity grades were increased if multiple hyperplastic lesions were present within the pancreas.

Table 26. Incidences of Neoplastic and Nonneoplastic Lesions of the Pancreas in Male and Female Rats in the Postweaning-only Two-year Feed Study of Di(2-ethylhexyl) Phthalate

\begin{tabular}{|c|c|c|c|c|c|}
\hline & 0 ppm & 300 ppm & $1,000 \mathrm{ppm}$ & $3,000 \mathrm{ppm}$ & $10,000 \mathrm{ppm}$ \\
\hline \multicolumn{6}{|l|}{ Male } \\
\hline $\mathbf{n}^{\mathbf{a}}$ & 49 & 50 & 50 & 50 & 50 \\
\hline Acinus, Hyperplasia ${ }^{\mathrm{b}}$ & $7 * *(2.6)^{\mathrm{c}}$ & $8(2.3)$ & $9(1.8)$ & $24 * *(3.3)$ & $26 * *(3.0)$ \\
\hline \multicolumn{6}{|l|}{ Acinar Adenoma $^{\mathrm{d}}$} \\
\hline Overall rate $^{e}$ & $1 / 49(2 \%)$ & $4 / 50(8 \%)$ & $5 / 50(10 \%)$ & $23 / 50(46 \%)$ & $30 / 50(60 \%)$ \\
\hline Adjusted rate $\mathrm{f}^{\mathrm{f}}$ & $2.4 \%$ & $9 \%$ & $10.7 \%$ & $49.9 \%$ & $64 \%$ \\
\hline Poly-3 test ${ }^{\mathrm{g}}$ & $\mathrm{p}<0.001$ & $\mathrm{p}=0.202$ & $\mathrm{p}=0.131$ & $\mathrm{p}<0.001$ & $\mathrm{p}<0.001$ \\
\hline \multicolumn{6}{|l|}{ Acinar Carcinoma ${ }^{\mathrm{h}}$} \\
\hline Overall rate & $0 / 49(0 \%)$ & $1 / 50(2 \%)$ & $0 / 50(0 \%)$ & $1 / 50(2 \%)$ & $5 / 50(10 \%)$ \\
\hline Adjusted rate & $0 \%$ & $2.3 \%$ & $0 \%$ & $2.2 \%$ & $10.6 \%$ \\
\hline Poly-3 test & $\mathrm{p}<0.001$ & $\mathrm{p}=0.513$ & (e) & $\mathrm{p}=0.515$ & $\mathrm{p}=0.043$ \\
\hline \multicolumn{6}{|c|}{ Acinar Adenoma or Carcinoma (Combined) ${ }^{\mathrm{i}}$} \\
\hline Overall rate & $1 / 49(2 \%)$ & $5 / 50(10 \%)$ & $5 / 50(10 \%)$ & $23 / 50(46 \%)$ & $33 / 50(66 \%)$ \\
\hline Adjusted rate & $2.4 \%$ & $11.2 \%$ & $10.7 \%$ & $49.9 \%$ & $69.8 \%$ \\
\hline Poly-3 test & $\mathrm{p}<0.001$ & $\mathrm{p}=0.119$ & $\mathrm{p}=0.131$ & $\mathrm{p}<0.001$ & $\mathrm{p}<0.001$ \\
\hline \multicolumn{6}{|l|}{ Female } \\
\hline $\mathbf{n}$ & 50 & 50 & 50 & 50 & 47 \\
\hline Acinus, Hyperplasia & $0 * *$ & $1(2.0)$ & $1(1.0)$ & $1(1.0)$ & $5 *(3.0)$ \\
\hline \multicolumn{6}{|l|}{ Acinar Adenoma $^{\mathrm{j}}$} \\
\hline Overall rate & $0 / 50(0 \%)$ & $0 / 50(0 \%)$ & $0 / 50(0 \%)$ & $1 / 50(2 \%)$ & $1 / 47(2 \%)$ \\
\hline Adjusted rate & $0 \%$ & $0 \%$ & $0 \%$ & $2.3 \%$ & $2.5 \%$ \\
\hline Poly-3 test & (n) & (n) & (n) & (n) & (n) \\
\hline \multicolumn{6}{|l|}{ Acinar Carcinoma ${ }^{\mathrm{j}}$} \\
\hline Overall rate & $0 / 50(0 \%)$ & $0 / 50(0 \%)$ & $0 / 50(0 \%)$ & $0 / 50(0 \%)$ & $1 / 47(2 \%)$ \\
\hline Adjusted rate & $0 \%$ & $0 \%$ & $0 \%$ & $0 \%$ & $2.5 \%$ \\
\hline Poly-3 test & (n) & (n) & (n) & (n) & (n) \\
\hline
\end{tabular}




\begin{tabular}{lccccc}
\hline & $\mathbf{0 ~ p p m}$ & $\mathbf{3 0 0} \mathbf{~ p p m}$ & $\mathbf{1 , 0 0 0} \mathbf{~ p p m}$ & $\mathbf{3 , 0 0 0} \mathbf{~ p p m}$ & $\mathbf{1 0 , 0 0 0} \mathbf{~ p p m}$ \\
\hline Acinar Adenoma or Carcinoma (Combined) & & & & & \\
Overall rate & $0 / 50(0 \%)$ & $0 / 50(0 \%)$ & $0 / 50(0 \%)$ & $1 / 50(2 \%)$ & $2 / 47(4 \%)$ \\
Adjusted rate & $0 \%$ & $0 \%$ & $0 \%$ & $2.3 \%$ & $5 \%$ \\
Poly-3 test & $\mathrm{p}=0.038$ & $(\mathrm{e})$ & $(\mathrm{e})$ & $\mathrm{p}=0.505$ & $\mathrm{p}=0.219$ \\
\hline
\end{tabular}

Statistical significance for an exposure group indicates a significant pairwise test compared to the vehicle control group.

Statistical significance for the vehicle control group indicates a significant trend test.

*Statistically significant at $\mathrm{p} \leq 0.05$ by the Poly- 3 test; $* * \mathrm{p} \leq 0.01$.

(e) = value of statistic could not be computed; $(n)=$ no statistics were calculated.

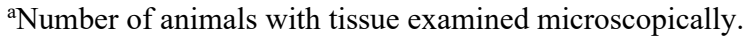

bNumber of animals with lesion.

${ }^{\mathrm{c}}$ Average severity grade of lesions in affected animals: $1=$ minimal, $2=$ mild, $3=$ moderate, $4=$ marked.

${ }^{\mathrm{d} H i s t o r i c a l ~ c o n t r o l ~ i n c i d e n c e ~ f o r ~ a l l ~ r o u t e s ~ o f ~ 2-y e a r ~ s t u d i e s ~(m e a n ~} \pm$ standard deviation): 60/488 (11.58\% $\pm 9.25 \%$ ); range: $0 \%$ to $28 \%$.

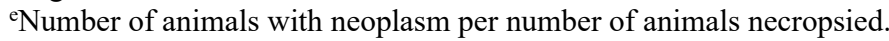

fPoly-3 estimated neoplasm incidence after adjustment for intercurrent mortality.

${ }^{g}$ Beneath the control incidence is the $p$ value associated with the trend test. Beneath the exposed group incidence are the $p$ values corresponding to pairwise comparisons between the vehicle control group and that exposed group. The Poly-3 test accounts for differential mortality in animals that do not reach study termination. A negative trend or a lower incidence in an exposure group is indicated by $\mathrm{N}$.

${ }^{h}$ Historical control incidence: $4 / 488(0.8 \% \pm 1.42 \%)$; range: $0 \%$ to $4 \%$.

${ }^{\mathrm{i}}$ Historical control incidence: $62 / 488(12.03 \% \pm 9.16 \%)$; range: $0 \%$ to $28 \%$.

jHistorical control incidence: $0 / 489$.

Testis: There was a positive trend in the incidence of testicular interstitial cell adenoma in male rats (Table 27). Interstitial cell adenomas were characterized by regions of increased Leydig cells, described as mostly uniform polyhedral cells with abundant eosinophilic, finely granular, or vacuolated cytoplasm, which exceeded the diameter of three seminiferous tubules. Circumferential compression of adjacent seminiferous tubules was observed occasionally.

There were significant increases in the incidences of germinal epithelium degeneration, bilateral germinal epithelium degeneration, testis edema, and bilateral testis edema in the 10,000 ppm group compared to the control males; there was a positive trend in the incidence of focal interstitial cell hyperplasia (Table 27). Germinal epithelium degeneration was recorded when one or more of the following features was present in tubules not adjacent to the rete testis: tubular vacuolation, partial depletion of germ cells, degenerating (multinucleated or apoptotic) germ cells, and disordered arrangement of the germ cell layers and/or seminiferous tubules completely devoid of germ cells and lined only by Sertoli cells. Germinal epithelium degeneration was scored using a four-grade severity scale: minimal (grade 1), up to $25 \%$ of at least one testis involved; mild (grade 2), $26 \%$ to $50 \%$ of at least one testis involved; moderate (grade 3 ), $51 \%$ to $75 \%$ of at least one testis involved; and marked (grade 4), rare to no normal seminiferous tubules in either testis were present (i.e., remaining seminiferous tubules solely lined by Sertoli cells).

Testis edema was characterized by the presence of acellular, finely granular or fibrillar pale eosinophilic material in the interstitium. In most animals, this finding was bilateral. A severity grade was determined by the amount of interstitial fluid. Generally, the severity of edema was higher in testes with reduced numbers of seminiferous tubules, because the interstitial fluid filled the intervening space. In some animals with reduced numbers of seminiferous tubules, however, the testis was collapsed and shrunken, leaving no interstitial space. 
Interstitial cell hyperplasia was scored using a four-grade severity scale: minimal (grade 1), when only a thin rim of interstitial cells or a cluster of cells one-fourth the size of a normal seminiferous tubule was present; mild (grade 2), when several such areas were present or one cluster was present that was one-half the size of a normal seminiferous tubule; moderate (grade 3), when a cluster three-fourths the size of a normal seminiferous tubule was present; and marked (grade 4), when a cluster of interstitial cells approached the diameter of a normal seminiferous tubule. The interstitial cells involved were frequently very elongated and flattened in profile.

Epididymis: There were significant increases in the incidences of bilateral hypospermia and bilateral epididymis duct exfoliated germ cell in the 10,000 ppm group relative to the control males (Table 27). Epididymis hypospermia was characterized by a reduced density of sperm in the lumen of the epididymal duct, often accompanied by luminal cell debris. Its severity was scored using a four-grade scale: minimal (grade 1), 25\%-50\% reduction of spermatozoa; mild (grade 2), 51\%-66\% reduction; moderate (grade 3), 67\%-80\% reduction; and marked (grade 4), $81 \%-100 \%$ reduction. The lesion of epididymis duct exfoliated germ cell was characterized by the presence of nondegenerate germ cells and debris in the epididymal lumen. This was often accompanied by depletion of germ cells from the seminiferous epithelium in testes diagnosed with germinal epithelium degeneration. 
Table 27. Incidences of Neoplastic and Nonneoplastic Lesions of the Testis and Epididymis in Male Rats in the Postweaning-only Two-year Feed Study of Di(2-ethylhexyl) Phthalate

\begin{tabular}{|c|c|c|c|c|c|}
\hline & O ppm & 300 ppm & 1,000 ppm & $3,000 \mathrm{ppm}$ & 10,000 ppm \\
\hline $\mathbf{n}^{\mathrm{a}}$ & 50 & 50 & 50 & 50 & 50 \\
\hline \multicolumn{6}{|l|}{ Testis } \\
\hline $\begin{array}{l}\text { Germinal epithelium, degeneration } \\
\text { (includes bilateral) }\end{array}$ & $31 * *(1.6)^{\mathrm{d}}$ & $25(1.7)$ & $21 *(1.5)$ & $22 *(1.6)$ & $50 * *(3.6)$ \\
\hline Edema (includes bilateral) & $27 * *(1.3)$ & $23(1.1)$ & $29(1.1)$ & $24(1.2)$ & $45 * *(2.7)$ \\
\hline $\begin{array}{l}\text { Interstitial cell, hyperplasia, focal } \\
\text { (includes bilateral) }\end{array}$ & $1 *(3.0)$ & $1(3.0)$ & 0 & $4(2.0)$ & $4(2.3)$ \\
\hline \multicolumn{6}{|l|}{ Epididymis } \\
\hline Hypospermia (includes bilateral) & $4 * *(3.8)$ & $4(3.5)$ & $4(3.3)$ & $3(3.7)$ & $43 * *(4.0)$ \\
\hline $\begin{array}{l}\text { Duct, exfoliated germ cell } \\
\text { (includes bilateral) }\end{array}$ & $2 * *(2.0)$ & $3(2.0)$ & $4(2.0)$ & $4(2.0)$ & $36 * *(1.4)$ \\
\hline \multicolumn{6}{|l|}{ Testis } \\
\hline \multicolumn{6}{|l|}{ Interstitial cell, adenoma ${ }^{e}$} \\
\hline Overall rate ${ }^{f}$ & $7 / 50(14 \%)$ & $3 / 50(6 \%)$ & $3 / 50(6 \%)$ & $6 / 50(12 \%)$ & $15 / 50(30 \%)$ \\
\hline Adjusted rate ${ }^{g}$ & $16.7 \%$ & $6.8 \%$ & $6.5 \%$ & $13.4 \%$ & $32.2 \%$ \\
\hline Poly-3 test ${ }^{\mathrm{h}}$ & $\mathrm{p}<0.001$ & $\mathrm{p}=0.135 \mathrm{~N}$ & $\mathrm{p}=0.119 \mathrm{~N}$ & $\mathrm{p}=0.451 \mathrm{~N}$ & $\mathrm{p}=0.073$ \\
\hline
\end{tabular}

Statistical significance for an exposure group indicates a significant pairwise test compared to the vehicle control group.

Statistical significance for the vehicle control group indicates a significant trend test.

*Statistically significant at $\mathrm{p} \leq 0.05$ by the Poly- 3 test; ** $\mathrm{p} \leq 0.01$.

${ }^{a}$ Number of animals with tissue examined microscopically.

${ }^{\mathrm{b}}$ Number of animals with lesion.

'Incidence reported is the combination of unilateral and bilateral lesions.

${ }^{\mathrm{d} A v e r a g e ~ s e v e r i t y ~ g r a d e ~ o f ~ l e s i o n s ~ i n ~ a f f e c t e d ~ a n i m a l s: ~} 1=$ minimal, $2=$ mild, $3=$ moderate, $4=$ marked.

eHistorical control incidence for all routes of 2-year studies (mean \pm standard deviation): $19 / 487(4.06 \% \pm 4.36 \%)$;

range: $0 \%$ to $14 \%$.

fNumber of animals with neoplasm per number of animals necropsied.

gPoly-3 estimated neoplasm incidence after adjustment for intercurrent mortality.

heneath the control incidence is the $\mathrm{p}$ value associated with the trend test. Beneath the exposed group incidence are the $\mathrm{p}$ values corresponding to pairwise comparisons between the vehicle control group and that exposed group. The Poly-3 test accounts for differential mortality in animals that do not reach study termination. A negative trend or a lower incidence in an exposure group is indicated by $\mathrm{N}$.

Uterus: There was a significant increase in the incidence of endometrium adenocarcinoma in the $10,000 \mathrm{ppm}$ group and a positive trend in the incidence of uterine squamous cell papilloma with increasing exposure concentration (Table 28). Uterus adenocarcinomas were typically poorly circumscribed and invaded the myometrium. The neoplastic epithelial cells formed solid nests, cords, papillary, or acinar structures. Uterine squamous cell papillomas were characterized by a neoplasm that arose from the surface epithelium with either a broad base or a delicate stalk. The epithelium was well differentiated and arranged in papillary, glandular, or tubular structures that were lined by cuboidal to columnar cells, one to two cell layers thick. The combined incidence of these was significantly increased in the 10,000 ppm group (Table 28).

There were significant increases in the incidences of uterine inflammation in the 300, 1,000, and 10,000 ppm groups, compared to the control group (Table 28). Uterine inflammation was characterized by a spectrum of changes from mostly mononuclear cells (recorded as chronic 
inflammation) to a mixture of mononuclear cells and neutrophils (recorded as chronic active inflammation); both diagnoses were considered a part of the same process.

Table 28. Incidences of Neoplastic and Nonneoplastic Lesions of the Uterus (Including Cervix) in Female Rats in the Postweaning-only Two-year Feed Study of Di(2-ethylhexyl) Phthalate

\begin{tabular}{|c|c|c|c|c|c|}
\hline & O ppm & 300 ppm & 1,000 ppm & 3,000 ppm & $10,000 \mathrm{ppm}$ \\
\hline $\mathbf{n}^{\mathbf{a}}$ & 50 & 50 & 50 & 50 & 49 \\
\hline Inflammation, Chronic ${ }^{\mathrm{b}}$ & $2(2.5)^{\mathrm{c}}$ & $9 *(2.0)$ & $6^{*}(2.5)$ & $8(2.0)$ & $8 *(3.0)$ \\
\hline Adenoma $^{\mathrm{d}}$ & 0 & 1 & 0 & 0 & 0 \\
\hline \multicolumn{6}{|l|}{ Adenocarcinoma $^{\mathrm{e}}$} \\
\hline Overall rate ${ }^{f}$ & $2 / 50(4 \%)$ & $2 / 50(4 \%)$ & $1 / 50(2 \%)$ & $4 / 50(8 \%)$ & $10 / 50(20 \%)$ \\
\hline Adjusted rate ${ }^{g}$ & $4.7 \%$ & $4.9 \%$ & $2.4 \%$ & $9 \%$ & $23.8 \%$ \\
\hline Poly-3 test ${ }^{\mathrm{h}}$ & $\mathrm{p}<0.001$ & $\mathrm{p}=0.678$ & $\mathrm{p}=0.508 \mathrm{~N}$ & $\mathrm{p}=0.352$ & $\mathrm{p}=0.011$ \\
\hline Squamous Cell Carcinoma ${ }^{i}$ & 0 & 1 & 0 & 2 & 1 \\
\hline Squamous Cell Papilloma & $0^{*}$ & 0 & 0 & 0 & 2 \\
\hline
\end{tabular}

(Includes Multiple)

Adenoma, Adenocarcinoma, Squamous Cell Carcinoma, or Squamous Cell Papilloma (Combined) ${ }^{\mathrm{k}}$

\begin{tabular}{lccccc} 
Overall rate & $2 / 50(4 \%)$ & $4 / 50(8 \%)$ & $1 / 50(2 \%)$ & $6 / 50(12 \%)$ & $13 / 50(26 \%)$ \\
Adjusted rate & $4.7 \%$ & $9.7 \%$ & $2.4 \%$ & $13.4 \%$ & $30.7 \%$ \\
Poly-3 test & $\mathrm{p}<0.001$ & $\mathrm{p}=0.315$ & $\mathrm{p}=0.508 \mathrm{~N}$ & $\mathrm{p}=0.145$ & $\mathrm{p}<0.001$ \\
\hline
\end{tabular}

Statistical significance for an exposure group indicates a significant pairwise test compared to the vehicle control group.

Statistical significance for the vehicle control group indicates a significant trend test.

*Statistically significant at $\mathrm{p} \leq 0.05$ by the Poly- 3 test.

anumber of animals with tissue examined microscopically.

${ }^{b}$ Number of animals with lesion.

${ }^{\mathrm{c}}$ Average severity grade of lesions in affected animals: $1=$ minimal, $2=$ mild, $3=$ moderate, $4=$ marked.

${ }^{\mathrm{d} H i s t o r i c a l ~ c o n t r o l ~ i n c i d e n c e ~ f o r ~ a l l ~ r o u t e s ~ o f ~ 2-y e a r ~ s t u d i e s ~(m e a n ~} \pm$ standard deviation): $1 / 350(0.29 \% \pm 0.76 \%)$; range: $0 \%$ to $2 \%$.

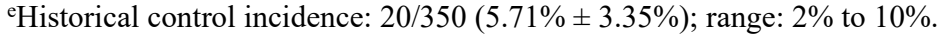

f Number of animals with neoplasm per number of animals necropsied.

gPoly-3 estimated neoplasm incidence after adjustment for intercurrent mortality.

${ }^{h}$ Beneath the control incidence is the $p$ value associated with the trend test. Beneath the exposed group incidence are the $p$ values corresponding to pairwise comparisons between the vehicle control group and that exposed group. The Poly-3 test accounts for differential mortality in animals that do not reach study termination. A negative trend or a lower incidence in an exposure group is indicated by $\mathrm{N}$.

${ }^{i}$ Historical control incidence: $2 / 350(0.57 \% \pm 1.51 \%)$; range: $0 \%$ to $4 \%$.

jHistorical control incidence: 0/350.

${ }^{k}$ Historical control incidence: $23 / 350(6.57 \% \pm 3.41 \%)$; range: $2 \%$ to $10 \%$.

Heart: There were significant increases in the incidences of heart valve fibrosis and heart valve thrombus in the 10,000 ppm male group relative to the control group (Table 29). Valve fibrosis was diagnosed when valves were expanded by fibrous connective tissue that was more densely eosinophilic than the loose lightly basophilic to amphophilic tissue of a normal heart valve. Heart valve thrombus was characterized by fibrin, admixed with variable numbers of blood cells, which covered the cardiac valves.

Bone Marrow: There was a significant increase in the incidence of bone marrow hypercellularity in the 1,000 and 10,000 ppm male groups relative to the control group (Table 29). Bone marrow 
hypercellularity was characterized by an increase in one or more hematopoietic cell lines, generally with a decrease in adipocytes.

Pituitary Gland: There was a significant increase in the incidence of pars distalis hypertrophy in the 10,000 ppm males compared to the control group (Table 29). Pars distalis hypertrophy was characterized by clusters of cells that were enlarged, with abundant amorphous amphophilic or pale eosinophilic cytoplasm and peripherally compressed nuclei ("signet ring" cells). A severity grade was assigned based on the numbers of affected cells.

Table 29. Incidences of Nonneoplastic Lesions of the Heart, Bone Marrow, and Pituitary Gland in Male Rats in the Postweaning-only Two-year Feed Study of Di(2-ethylhexyl) Phthalate

\begin{tabular}{lccccc}
\hline & $\mathbf{0 ~} \mathbf{~ p m}$ & $\mathbf{3 0 0} \mathbf{~ p p m}$ & $\mathbf{1 , 0 0 0} \mathbf{~ p p m}$ & $\mathbf{3 , 0 0 0} \mathbf{~ p p m}$ & $\mathbf{1 0 , 0 0 0} \mathbf{~ p p m}$ \\
\hline $\mathbf{n}^{\mathbf{a}}$ & 50 & 50 & 50 & 50 & 50 \\
\hline $\begin{array}{l}\text { Male } \\
\text { Heart }\end{array}$ & & & & & \\
$\quad$ Valve, fibrosis & & & & & \\
$\quad$ Valve, thrombus & $2^{* *}(1.5)^{\mathrm{c}}$ & 0 & 0 & $1(1.0)$ & $9^{*}(1.9)$ \\
Bone Marrow & $0^{* *}$ & 0 & 0 & $2(2.5)$ & $6^{*}(1.8)$ \\
$\quad$ Hypercellularity & & & & & \\
$\quad \begin{array}{l}\text { Pituitary Gland } \\
\quad \text { Pars distalis, hypertrophy }\end{array}$ & $8^{* *}(2.1)$ & $22(2.1)$ & $30^{*}(1.8)$ & $25(1.8)$ & $34^{* *}(1.9)$ \\
\hline
\end{tabular}

Statistical significance for an exposure group indicates a significant pairwise test compared to the vehicle control group.

Statistical significance for the vehicle control group indicates a significant trend test.

*Statistically significant at $\mathrm{p} \leq 0.05$ by the Poly- 3 test; $* * \mathrm{p} \leq 0.01$.

a Number of animals examined microscopically.

${ }^{b}$ Number of animals with lesion.

${ }^{\mathrm{c}}$ Average severity grade of lesions in affected animals: $1=$ minimal; $2=$ mild; $3=$ moderate; $4=$ marked.

The toxicological significance of other lesion findings is unknown as the response was considered either marginal, not dose-responsive, and/or decreased (potentially due to lower body weights). (Appendix H). In males, these lesions included heart cardiomyopathy and parathyroid gland diffuse hyperplasia. In females, these lesions included: heart cardiomyopathy; uterus endometrium metaplasia; mammary gland fibroadenoma; mammary gland fibroma, fibroadenoma, or adenoma; mammary gland fibroma, fibroadenoma, carcinoma, or adenoma; nose, olfactory epithelium, hyaline droplet accumulation; pituitary gland pars distalis adenoma; thyroid gland C-cell hyperplasia; and thyroid gland C-cell adenoma or carcinoma.

\section{Comparative Carcinogenic Benchmark Dose Analysis}

Exposure-related neoplastic lesions were further assessed via benchmark dose (BMD) analyses. Daily doses for each exposure group were calculated using time-weighted averages of postweaning feed consumption and corresponding chemical intake during the 2-year exposure period for each study. All available dichotomous models in U.S. EPA's BMD Software (BMDS version 3.1.2) ${ }^{169}$ were fit to the adjusted incidence data for assessed neoplastic lesions. Model fit was assessed by a chi-square goodness-of-fit test, visual inspection of the respective plots of observed versus predicted values from the various models, and Akaike information criterion 
(AIC) values (Appendix F). A benchmark response (BMR) of 0.1 , corresponding to a $10 \%$ extra risk of a DEHP carcinogenic response, was used to determine benchmark doses. Benchmark doses (i.e., BMD $\mathrm{BM}_{10}$ [BMD corresponding to a $10 \%$ extra risk] and BMDL $\mathrm{B}_{10}[95 \%$ lower bound on the BMD corresponding to a $10 \%$ extra risk]) were determined for incidences of hepatocellular adenoma or carcinoma (combined), pancreatic acinar adenoma or carcinoma (combined), testicular interstitial cell adenoma, and uterine (including cervix) adenoma, adenocarcinoma, squamous cell carcinoma, or squamous cell papilloma (combined). The BMD 10 and BMDL 10 were calculated separately for the perinatal and postweaning study (Study 1) and for the postweaning-only study (Study 2).

Higher adjusted incidences of hepatocellular adenoma or carcinoma (combined) occurred in the 3,000 and $10,000 \mathrm{ppm}$ male rats exposed during the perinatal and postweaning periods $(6.7 \%$ and $30.6 \%$ ), relative to postweaning-only exposure (2.2\% and 25.6\%) (Table 30). A probit model provided the best relative model fit for the adjusted rates of hepatocellular adenoma or carcinoma (combined) in the perinatal and postweaning study (Figure 14A). Using this model, a BMD 10 of $382.90 \mathrm{mg} / \mathrm{kg} /$ day was estimated for hepatocellular adenoma or carcinoma (combined) in male rats. A multistage degree 4 model provided the best relative model fit for the adjusted rates of hepatocellular adenoma or carcinoma (combined) in the postweaning-only study (Figure 14B). Using this model, a BMD 10 of $434.41 \mathrm{mg} / \mathrm{kg} /$ day was estimated for hepatocellular adenoma or carcinoma (combined) observed in male rats.

Table 30. Adjusted Incidence Data and Benchmark Dose Modeling for Select Neoplasms in Male Rats in the Two-year Feed Studies of Di(2-Ethylhexyl) Phthalate

\begin{tabular}{|c|c|c|c|c|c|c|c|c|}
\hline Neoplasm & $\begin{array}{c}\mathbf{0} \\
\text { ppm }\end{array}$ & $\begin{array}{c}300 \\
\text { ppm }\end{array}$ & $\begin{array}{l}1,000 \\
\text { ppm }\end{array}$ & $\begin{array}{l}\text { 3,000 } \\
\text { ppm }\end{array}$ & $\begin{array}{c}10,000 \\
\text { ppm }\end{array}$ & $\begin{array}{c}\text { BMD }_{10} \\
(\mathrm{mg} / \mathrm{kg} / \text { day })\end{array}$ & $\begin{array}{c}\text { BMDL } 10 \\
(\mathrm{mg} / \mathrm{kg} / \mathrm{day})\end{array}$ & Model \\
\hline \multicolumn{9}{|c|}{ Perinatal and Postweaning Study (Study 1) } \\
\hline DEHP Intake (mg/kg/day) & 0 & 17.6 & 57.5 & 188.5 & 678.3 & - & - & - \\
\hline $\begin{array}{l}\text { Hepatocellular Adenoma } \\
\text { or Carcinoma (Combined) }\end{array}$ & $2.6 \%$ b & $2.4 \%$ & $0 \%$ & $6.7 \%$ & $30.6 \%$ & $382.90^{c}$ & $306.05^{\mathrm{c}}$ & Probit \\
\hline $\begin{array}{l}\text { Pancreatic Acinar } \\
\text { Adenoma or Carcinoma } \\
(\text { Combined })^{\mathrm{d}}\end{array}$ & $26 \%$ & $16.6 \%$ & $16.9 \%$ & $81.2 \%$ & $62.5 \%$ & $85.92^{\mathrm{c}}$ & $56.78^{\mathrm{c}}$ & $\begin{array}{l}\text { Dichotomous } \\
\text { Hill }\end{array}$ \\
\hline \multicolumn{9}{|c|}{ Postweaning-only Study (Study 2) } \\
\hline DEHP Intake (mg/kg/day) & 0 & 16.8 & 53.5 & 169.9 & 602.3 & - & - & - \\
\hline $\begin{array}{l}\text { Hepatocellular Adenoma } \\
\text { or Carcinoma (Combined) }\end{array}$ & $0 \%$ & $4.5 \%$ & $0 \%$ & $2.2 \%$ & $25.6 \%$ & 434.41 & 263.52 & $\begin{array}{l}\text { Multistage } \\
\text { degree } 4\end{array}$ \\
\hline 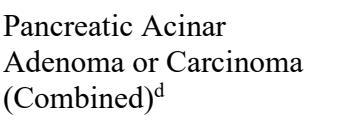 & $2.4 \%$ & $11.2 \%$ & $10.7 \%$ & $49.9 \%$ & $69.8 \%$ & 30.99 & 20.20 & Log-logistic \\
\hline $\begin{array}{l}\text { Testis Interstitial Cell } \\
\text { Adenoma }^{\mathrm{e}}\end{array}$ & $16.7 \%$ & $6.8 \%$ & $6.5 \%$ & $13.4 \%$ & $32.2 \%$ & 366.69 & 164.41 & $\begin{array}{l}\text { Multistage } \\
\text { degree } 4\end{array}$ \\
\hline
\end{tabular}

$\mathrm{BMD}_{10}=$ benchmark dose corresponding to a $10 \%$ extra risk; $\mathrm{BMDL}_{10}=95 \%$ lower bound on the benchmark dose corresponding to a $10 \%$ extra risk.

${ }^{a}$ Historical control incidence for all routes of 2-year studies (mean \pm standard deviation): 4/489 $(0.89 \% \pm 1.06 \%)$; range: $0 \%$ to $2 \%$.

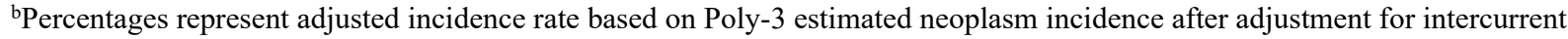
mortality.

${ }^{\mathrm{c}} \mathrm{BMD}$ models excluded incidences in the $10,000 \mathrm{ppm}$ group due to the nonmonotonicity of the dose response.

${ }^{\mathrm{d}}$ Historical control incidence for all routes of 2-year studies (mean \pm standard deviation): 62/488 (12.03\% $\left.\pm 9.16 \%\right)$; range: $0 \%$ to $28 \%$.

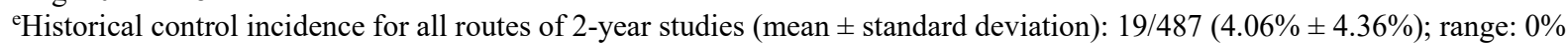
to $14 \%$. 
A.

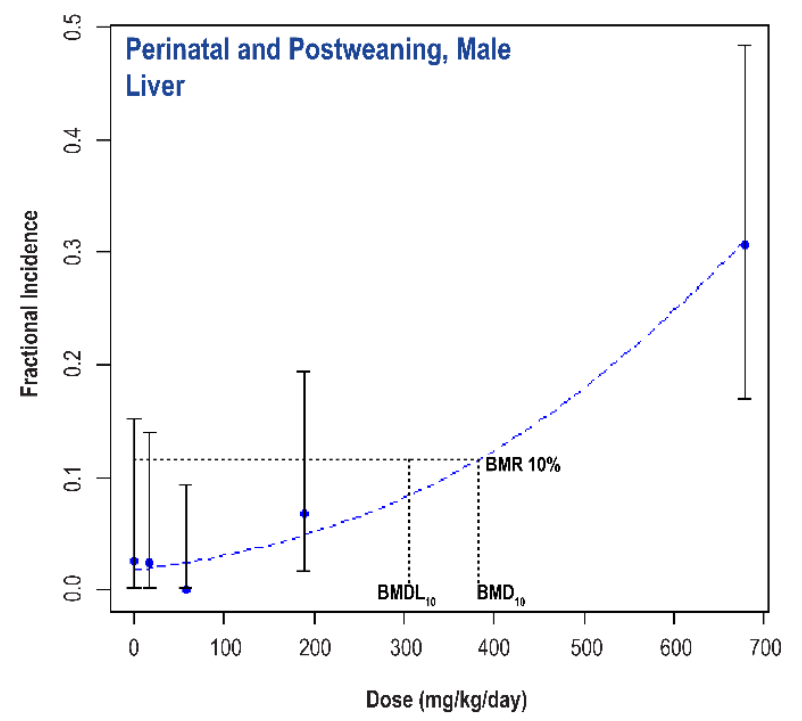

B.

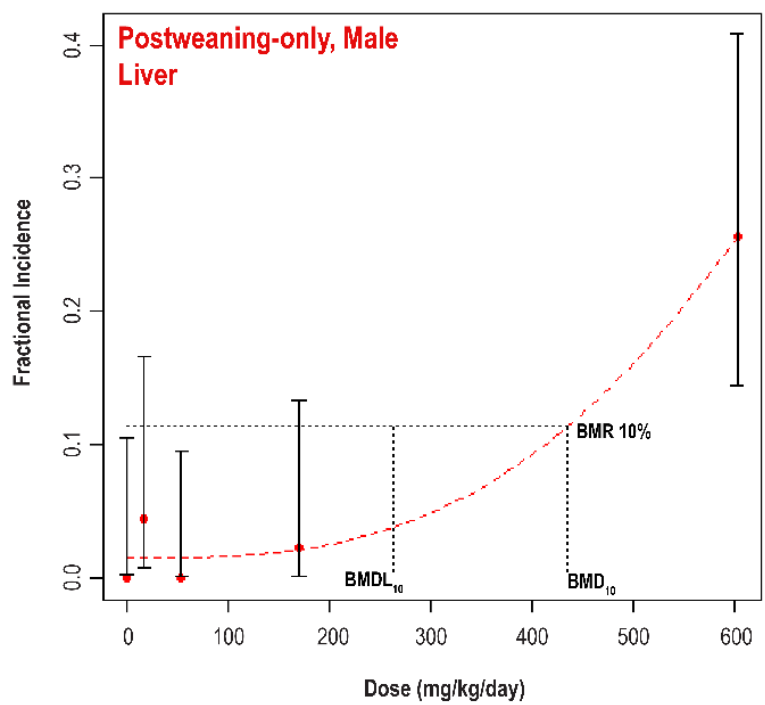

Figure 14. Benchmark Dose Modeling Results for Hepatocellular Adenoma or Carcinoma (Combined) in Male Rats

Frequentist (A) probit model (perinatal and postweaning; Study 1) and (B) multistage degree 4 model (postweaning-only; Study 2) with BMR of $10 \%$ extra risk for the $\mathrm{BMD}_{10}$ and 0.95 lower confidence limit for the BMDL 10 for the incidence of hepatocellular adenoma or carcinoma (combined) in male rats.

$\mathrm{BMR}=$ benchmark response; $\mathrm{BMD}_{10}=$ benchmark dose corresponding to a $10 \%$ extra risk; $\mathrm{BMDL}_{10}=95 \%$ lower bound on the benchmark dose corresponding to a $10 \%$ extra risk.

A higher adjusted incidence of hepatocellular adenoma or carcinoma (combined) occurred in $3,000 \mathrm{ppm}$ female rats exposed during the perinatal and postweaning periods (20.9\%) relative to postweaning-only exposure (2.3\%) (Table 31 ). A log-logistic model provided the best relative model fit for the adjusted rates of hepatocellular adenoma or carcinoma (combined) in the perinatal and postweaning study (Figure 15A). Using this model, a BMD 10 of $122.95 \mathrm{mg} / \mathrm{kg} / \mathrm{day}$ was estimated for hepatocellular adenoma or carcinoma (combined) in female rats. A multistage degree 4 model provided the best relative model fit for the adjusted rates of hepatocellular adenoma or carcinoma (combined) in the postweaning-only study (Figure 15B). Using this model, a $\mathrm{BMD}_{10}$ of $383.63 \mathrm{mg} / \mathrm{kg} /$ day was estimated for hepatocellular adenoma or carcinoma (combined) in female rats. 
Table 31. Adjusted Incidence Data and Benchmark Dose Modeling for Select Neoplasms in Female Rats in the Two-year Feed Studies of Di(2-Ethylhexyl) Phthalate

\begin{tabular}{|c|c|c|c|c|c|c|c|c|}
\hline Neoplasm & $\begin{array}{c}0 \\
\text { ppm }\end{array}$ & $\begin{array}{c}300 \\
\text { ppm }\end{array}$ & $\begin{array}{l}1,000 \\
\text { ppm }\end{array}$ & $\begin{array}{l}3,000 \\
\text { ppm }\end{array}$ & $\begin{array}{l}10,000 \\
\text { ppm }\end{array}$ & $\begin{array}{c}\mathrm{BMD}_{10} \\
\text { (mg/kg/day) }\end{array}$ & $\begin{array}{c}\text { BMDL } 10 \\
\text { (mg/kg/day) }\end{array}$ & Model \\
\hline \multicolumn{9}{|c|}{ Perinatal and Postweaning Study (Study 1) } \\
\hline DEHP Intake (mg/kg/day) & 0 & 17.9 & 61.7 & 195.6 & 772.3 & - & - & - \\
\hline $\begin{array}{l}\text { Hepatocellular Adenoma or } \\
\text { Carcinoma (Combined) }^{\mathrm{a}}\end{array}$ & $2.4 \% \mathrm{~b}$ & $0 \%$ & $11.8 \%$ & $20.9 \%$ & $35.4 \%$ & 122.95 & 79.74 & $\begin{array}{l}\text { Log- } \\
\text { logistic }\end{array}$ \\
\hline $\begin{array}{l}\text { Uterus (Including Cervix) } \\
\text { Adenocarcinoma, Adenoma, } \\
\text { Squamous Cell Carcinoma, or } \\
\text { Squamous Cell Papilloma } \\
(\text { Combined) }\end{array}$ & $7.0 \%$ & $2.4 \%$ & $2.4 \%$ & $7.0 \%$ & $19.0 \%$ & 594.19 & 432.23 & Logistic \\
\hline \multicolumn{9}{|c|}{ Postweaning-only Study (Study 2) } \\
\hline DEHP Intake (mg/kg/day) & 0 & 17.2 & 59.5 & 177.1 & 646.3 & - & - & - \\
\hline $\begin{array}{l}\text { Hepatocellular Adenoma or } \\
\text { Carcinoma (Combined) }\end{array}$ & $0 \%$ & $0 \%$ & $2.4 \%$ & $2.3 \%$ & $33.7 \%$ & 383.63 & 207.99 & $\begin{array}{l}\text { Multistage } \\
\text { degree } 4\end{array}$ \\
\hline $\begin{array}{l}\text { Uterus (Including Cervix) } \\
\text { Adenocarcinoma, Adenoma, } \\
\text { Squamous Cell Carcinoma, or } \\
\text { Squamous Cell Papilloma } \\
\text { (Combined) }^{c}\end{array}$ & $4.7 \%$ & $9.7 \%$ & $2.4 \%$ & $13.4 \%$ & $30.7 \%$ & 324.15 & 249.01 & Probit \\
\hline 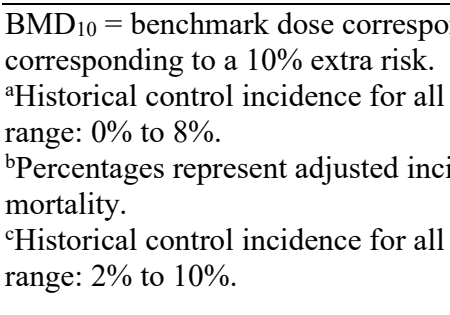 & $\begin{array}{l}\text { to a } \\
\text { e rate } 2 \\
\text { es of } 2 \text {. }\end{array}$ & $\begin{array}{l}\% \text { extr } \\
\text { ear stu } \\
\text { sed on }\end{array}$ & $\begin{array}{l}\text { isk; BM } \\
\text { s (mean } \\
\text { oly-3 est }\end{array}$ & $\begin{array}{l}\mathrm{DL}_{10}= \\
\pm \text { stanc } \\
\text { timated }\end{array}$ & $\begin{array}{l}\text { o low } \\
\text { devia } \\
\text { plasm }\end{array}$ & $\begin{array}{l}\text { bound on the } \\
\text { incidence after } \\
16 / 489\end{array}$ & $\% \pm 2.8 \%)$ & rcurrent \\
\hline
\end{tabular}


A.

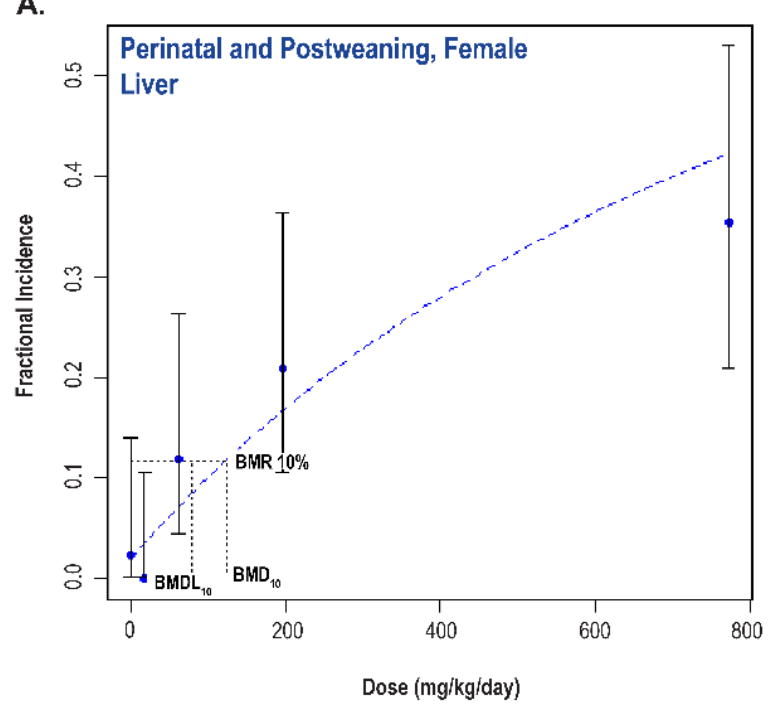

B.

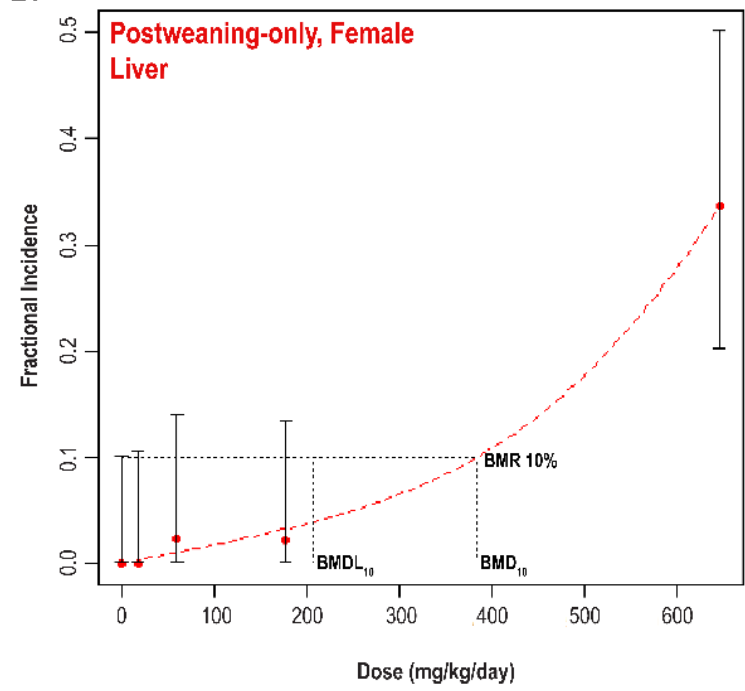

Figure 15. Benchmark Dose Modeling Results for Hepatocellular Adenoma or Carcinoma (Combined) in Female Rats

Frequentist (A) log-logistic model (perinatal and postweaning; Study 1) and (B) multistage degree 4 model (postweaning-only; Study 2) with BMR of $10 \%$ extra risk for the BMD 10 and 0.95 lower confidence limit for the BMDL 10 for the incidence of hepatocellular adenoma or carcinoma (combined) in female rats.

$\mathrm{BMR}=$ benchmark response; $\mathrm{BMD}_{10}=$ benchmark dose corresponding to a $10 \%$ extra risk; $\mathrm{BMDL}_{10}=95 \%$ lower bound on the benchmark dose corresponding to a $10 \%$ extra risk.

A higher adjusted incidence of pancreatic acinar adenoma or carcinoma (combined) occurred in 3,000 ppm male rats exposed during the perinatal and postweaning periods $(81.2 \%)$, relative to postweaning-only exposure (49.9\%); however, incidences in the 10,000 ppm group were similar between the two studies (62.5\% versus 69.8\%, respectively) (Table 30). A dichotomous Hill model provided the best relative model fit for the adjusted rates of pancreatic acinar adenoma or carcinoma (combined) in the perinatal and postweaning study (Figure 16A). Using this model, a $\mathrm{BMD}_{10}$ of $85.92 \mathrm{mg} / \mathrm{kg} /$ day was estimated for pancreatic acinar adenoma or carcinoma (combined) in male rats (Table 30). A log-logistic model provided the best relative model fit for the adjusted rates of pancreatic acinar adenoma or carcinoma (combined) in the postweaningonly study (Figure 16B). Using this model, a BMD 10 of $30.99 \mathrm{mg} / \mathrm{kg} /$ day was estimated for pancreatic acinar adenoma or carcinoma (combined) in male rats (Table 30). 


\section{A.}

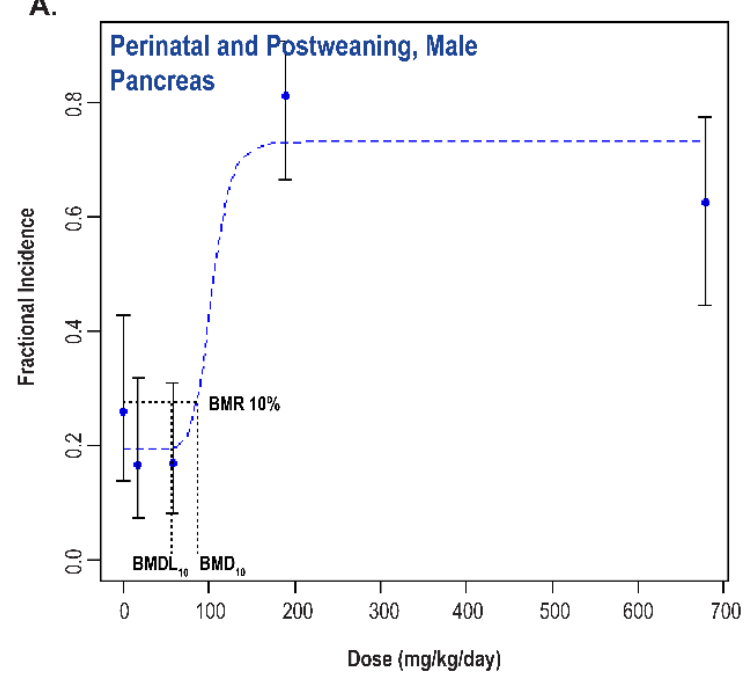

B.

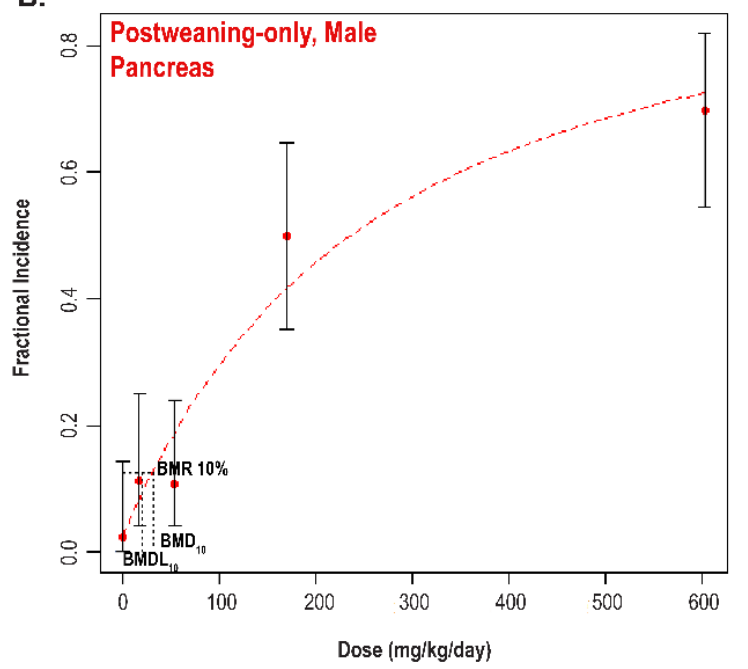

Figure 16. Benchmark Dose Modeling Results for Pancreatic Acinar Adenoma or Carcinoma (Combined) in Male Rats

Frequentist (A) dichotomous Hill model (perinatal and postweaning; Study 1) and (B) log-logistic model (postweaning-only; Study 2) with BMR of $10 \%$ extra risk for the $\mathrm{BMD}_{10}$ and 0.95 lower confidence limit for the BMDL $\mathrm{B}_{10}$ for the incidence of pancreatic acinar adenoma or carcinoma (combined) in male rats.

$\mathrm{BMR}=$ benchmark response; $\mathrm{BMD}_{10}=$ benchmark dose corresponding to a $10 \%$ extra risk; $\mathrm{BMDL}_{10}=95 \%$ lower bound on the benchmark dose corresponding to a $10 \%$ extra risk.

The incidences of pancreatic acinar adenoma or carcinoma (combined) in female rats were not amenable to BMD modeling. These lesions are considered rare in female rats and were only observed at adjusted rates up to 5\% in any single exposed group across both studies. Therefore, an estimated BMR corresponding to $10 \%$ extra risk would be greater than the maximum exposure concentration used in the study.

A higher adjusted incidence of testicular interstitial cell adenoma occurred only in 10,000 ppm male rats exposed during the postweaning period (32.3\%), relative to perinatal and postweaning exposure (14.1\%) (Table 16, Table 27, Table 32). Although there was no exposure-related response in the testis from the perinatal and postweaning study (Study 1), there was a marginal response in the testis with postweaning-only exposure (Study 2). A multistage degree 4 model provided the best relative model fit for the adjusted rates of testicular interstitial cell adenoma in the postweaning-only study (Table 32; Figure 16; Appendix H). Using this model, a BMD 10 of $366.69 \mathrm{mg} / \mathrm{kg} /$ day was estimated for testicular interstitial cell adenoma in male rats. 
Table 32. Incidence Data and Benchmark Dose Modeling Results for Testicular Interstitial Cell Adenoma in Male Rats in the Postweaning-only Two-year Feed Study of Di(2-ethylhexyl) Phthalate

\begin{tabular}{|c|c|c|c|c|c|c|c|c|}
\hline Neoplasm & 0 ppm & 300 ppm & 1,000 ppm & 3,000 ppm & 10,000 ppm & $\begin{array}{c}\text { BMD }_{10} \\
\text { (mg/kg/day) }\end{array}$ & $\begin{array}{c}\text { BMDL }_{10} \\
\text { (mg/kg/day) }\end{array}$ & Model \\
\hline \multicolumn{9}{|c|}{ Postweaning-only Study (Study 2) } \\
\hline $\mathbf{n}^{\mathbf{a}}$ & 50 & 50 & 50 & 50 & 50 & & & \\
\hline \multicolumn{9}{|c|}{ Testicular Interstitial Cell Adenoma ${ }^{\mathrm{b}}$} \\
\hline Overall rate ${ }^{c}$ & $7 / 50(14 \%)$ & $3 / 50(6 \%)$ & $3 / 50(6 \%)$ & $6 / 50(12 \%)$ & $15 / 50(30 \%)$ & 366.69 & 164.41 & Multistage degree 4 \\
\hline Adjusted rate ${ }^{\mathrm{d}}$ & $16.7 \%$ & $6.8 \%$ & $6.5 \%$ & $13.4 \%$ & $32.3 \%$ & & & \\
\hline Poly-3 test $\mathrm{t}^{\mathrm{e}}$ & $\mathrm{p}<0.001$ & $\mathrm{p}=0.135 \mathrm{~N}$ & $\mathrm{p}=0.119 \mathrm{~N}$ & $\mathrm{p}=0.451 \mathrm{~N}$ & $\mathrm{p}=0.073$ & & & \\
\hline
\end{tabular}

$\mathrm{BMD}_{10}=$ benchmark dose corresponding to a $10 \%$ extra risk; BMDL10 $=95 \%$ lower bound on the benchmark dose corresponding to a $10 \%$ extra risk.

aNumber of animals with tissue examined microscopically.

${ }^{b}$ Historical control incidence for all routes of 2-year studies (mean \pm standard deviation): $19 / 487(4.06 \% \pm 4.36 \%$ ); range: $0 \%$ to $14 \%$.

cNumber of animals with neoplasm per number of animals necropsied.

dPoly-3 estimated neoplasm incidence after adjustment for intercurrent mortality.

${ }^{\mathrm{e} B e n e a t h}$ the control incidence is the $\mathrm{p}$ value associated with the trend test. Beneath the exposed group incidence are the $\mathrm{p}$ values corresponding to pairwise comparisons between the vehicle control group and that exposed group. The Poly-3 test accounts for differential mortality in animals that do not reach terminal euthanasia. A negative trend or a lower incidence in an exposure group is indicated by $\mathrm{N}$. 
Higher adjusted incidences of uterine (including cervix) adenocarcinoma, adenoma, squamous cell carcinoma, or squamous cell papilloma (combined) occurred in 3,000 and 10,000 ppm female rats exposed postweaning-only (13.4\% and 30.7\%, respectively), relative to exposure during the perinatal and postweaning periods (7\% and 19\%, respectively) (Table 31). A logistic model provided the best relative model fit for the adjusted rates of uterine neoplasms in the perinatal and postweaning study (Figure 17A). Using this model, a BMD 10 of $594.19 \mathrm{mg} / \mathrm{kg} / \mathrm{day}$ was estimated for uterine (including cervix) adenocarcinoma, adenoma, squamous cell carcinoma, or squamous cell papilloma (combined) in female rats (Table 31). A probit model provided the best relative model fit for the adjusted rates of uterine (including cervix) adenocarcinoma, adenoma, squamous cell carcinoma, or squamous cell papilloma (combined) in the postweaning-only study (Figure 17B). Using this model, a BMD 10 of $324.15 \mathrm{mg} / \mathrm{kg} / \mathrm{day}$ was estimated for uterine (including cervix) adenocarcinoma, adenoma, squamous cell carcinoma, or squamous cell papilloma (combined) in female rats (Table 31).

A.

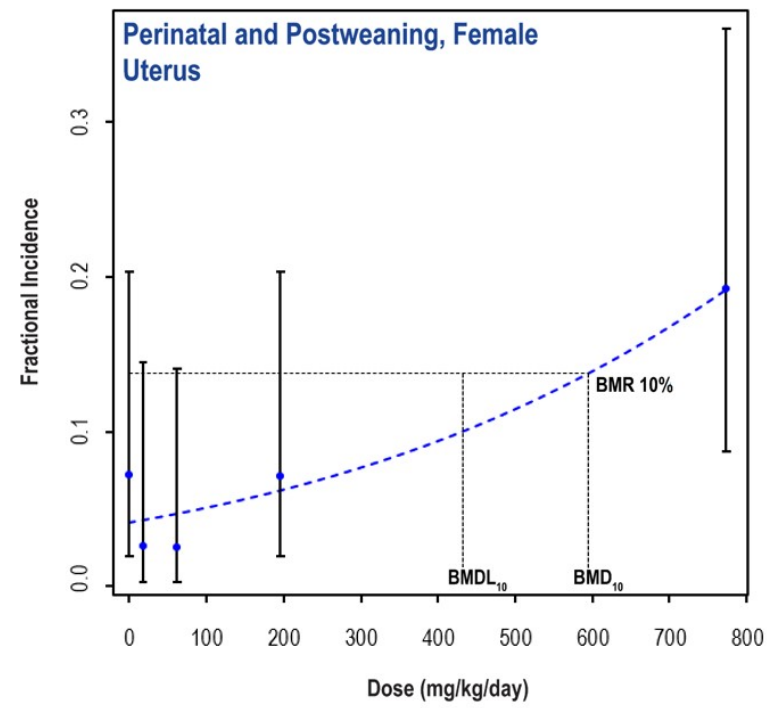

B.

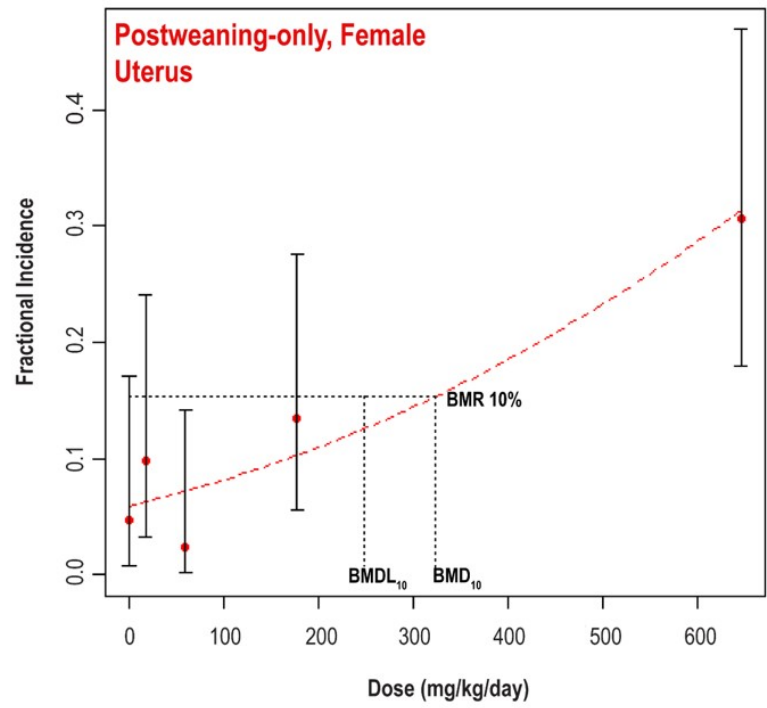

Figure 17. Benchmark Dose Modeling Results for Uterine Adenocarcinoma, Adenoma, Squamous Cell Carcinoma, or Squamous Cell Papilloma (Combined) in Female Rats

Frequentist (A) logistic model (perinatal and postweaning; Study 1) and (B) probit model (postweaning-only; Study 2) with BMR of $10 \%$ extra risk for the $\mathrm{BMD}_{10}$ and 0.95 lower confidence limit for the $\mathrm{BMDL}_{10}$ for the incidence of uterine adenocarcinoma, adenoma, squamous cell carcinoma, or squamous cell papilloma (combined) in female rats.

$\mathrm{BMR}=$ benchmark response $\mathrm{BMD}_{10}=$ benchmark dose corresponding to a $10 \%$ extra risk; $\mathrm{BMDL}_{10}=95 \%$ lower bound on the benchmark dose corresponding to a $10 \%$ extra risk.

\section{Genetic Toxicology}

DEHP was tested in a variety of genotoxicity assays in vitro and in vivo; most results were negative. DEHP (100-10,000 $\mu$ g/plate) was tested in six independent bacterial mutation assays using a variety of strains of Salmonella typhimurium (TA100, TA1535, TA1537, TA97, and TA98) and exogenous metabolic activation systems (induced hamster, rat, or mouse liver S9 plus cofactors); results from all bacterial assays were negative (Appendix $\mathrm{H}$ ). ${ }^{149}$ A single mouse 
lymphoma gene mutation assay was conducted with DEHP $(0.125-3.0 \mu \mathrm{L} / \mathrm{mL})$ and was negative overall, with and without induced rat liver S9 mix (Appendix H). In three independent studies, no increases in chromosomal aberrations were observed in Chinese hamster ovary (CHO) cells exposed to DEHP (concentrations up to $5,000 \mu \mathrm{g} / \mathrm{mL}$ ) with or without induced rat liver S9 (Appendix H). ${ }^{153}$ In a series of nine in vitro sister chromatid exchange (SCE) tests conducted in $\mathrm{CHO}$ cells with and without S9, DEHP produced positive responses in four tests, equivocal results in three, and negative results in two (Appendix H). ${ }^{153}$ All of the increases in SCEs judged to be positive or equivocal were observed only in the absence of S9 and at concentrations of DEHP that induced severe cell cycle delay, necessitating longer incubation prior to cell harvesting. The level of cytotoxicity and the extended incubation times might have contributed to the increased SCE levels observed in these seven studies, rather than the SCE reflecting a direct interaction of DEHP with chromosomal DNA. DEHP was tested for induction of sex-linked recessive lethal mutations in male Drosophila melanogaster in two independent studies, one using adult injection and one using larval feeding as the route of exposure; both studies yielded negative results (Appendix $\mathrm{H}) .{ }^{158 ; 159}$

In vivo, no significant increases in chromosomal aberrations were observed in bone marrow cells of female B6C3F1 mice following exposure to DEHP $(3,000-12,000 \mathrm{ppm})$ in dosed feed for 14 days (Table D-1). DEHP was tested in three independent erythrocyte micronucleus tests and produced varying results. In one test, $\mathrm{B} 6 \mathrm{C} 3 \mathrm{~F} 1$ female mice were exposed to $\mathrm{DEHP}(3,000-$ $12,000 \mathrm{ppm}$ ) in dosed feed for 14 days; results were judged to be equivocal overall — the response was negative in the immature erythrocyte population (polychromatic erythrocytes) and positive in the mature erythrocyte population (normochromatic erythrocytes) (Table D-2). In a second test, DEHP (1,500-6,000 ppm) induced an equivocal response in male TgAC (FVB/N) mice and a positive response in female $\operatorname{TgAC}(\mathrm{FVB} / \mathrm{N})$ mice following exposure via dosed feed for 26 weeks (Table D-3). Another 26-week exposure test in TgAC (FVB/N) mice used dermal application of DEHP (100-400 $\mathrm{mg} / \mathrm{kg} /$ day) and produced negative results in both male and female mice (Table D-4). 


\section{Discussion}

Di(2-ethylhexyl) phthalate (DEHP) is a plasticizer to which humans are exposed, as evidenced by detection of DEHP metabolites in serum and urine samples. The presence of DEHP metabolites in human amniotic fluid samples shows exposure occurs in utero. Rodent studies report that DEHP produces adverse effects on the developing male rat reproductive tract and induces hepatic, pancreatic, and testicular neoplasms. Prior to the current studies, data were insufficient to assess whether developmental exposure would alter lifetime DEHP carcinogenic risk. To address this knowledge gap, the National Toxicology Program (NTP) conducted two 2year bioassays with DEHP administered in feed to Sprague Dawley (Hsd:Sprague Dawley ${ }^{\circledR}$ $\mathrm{SD}^{\circledR}$ ) rats to evaluate whether neoplasm incidence during lifetime exposure that included the perinatal period (gestation and lactation) would increase the incidence of neoplasms or lead to the appearance of different neoplasm types relative to chronic exposure initiated in early adulthood.

In the perinatal and postweaning study (Study 1), exposure was associated with significantly decreased maternal mean body weights during gestation and lactation in the 10,000 ppm group compared to the control group, with the magnitude of the effect increasing throughout the perinatal period. This effect was attributed to significantly decreased body weight gain during gestation, likely in part due to cumulative effects of reduced maternal feed consumption (g/animal/day) throughout the gestation and lactation period in the 10,000 ppm group.

Estimated DEHP intake increased in proportion to exposure concentration, with the exception of $10,000 \mathrm{ppm}$ dams during lactation (lactation days [LDs] 1-14), for which significantly decreased feed consumption resulted in a less-than-proportional higher intake. In gestation day (GD) 18 dams, the mean concentration of the DEHP metabolite, mono(2-ethylhexyl) phthalate (MEHP), increased with exposure concentration although the increase was more than proportional (63-fold increase in plasma concentration versus 30 -fold increase in DEHP intake $[\mathrm{mg}$ DEHP $/ \mathrm{kg}$ body weight/day or $\mathrm{mg} / \mathrm{kg} /$ day] during gestation from lowest [300 ppm] to highest [10,000 ppm] exposure groups). Amniotic fluid and fetus concentrations of MEHP increased 23- and 46-fold, respectively, from the lowest to highest exposure groups (300 to 10,000 ppm). Gestational transfer of MEHP from the dam to the fetus was moderate.

MEHP concentrations in dam plasma on GD 18 at the lowest exposure concentration were $630 \mathrm{ng} / \mathrm{mL}$, approximately 60 -fold higher than the median blood MEHP concentration of $10.4 \mathrm{ng} / \mathrm{mL}$ observed in pregnant women in the Hokkaido Study Sapporo Cohort. ${ }^{203}$ Moreover, the GD 18 MEHP concentration measured in amniotic fluid at the lowest exposure concentration was $73.4 \mathrm{ng} / \mathrm{mL}$, which is 28-fold higher than the upper 95th percentile of MEHP levels measured in human amniotic fluid samples. ${ }^{26}$ Additional studies have detected MEHP in human amniotic fluid and cord blood plasma samples, indicating that DEHP or its metabolites cross the placental barrier and result in exposure to the developing conceptus. ${ }^{204-206}$ DEHP levels in control feed were below the limit of detection (1.27 ppm) of the analytical method; however, detectable levels of MEHP were measured in control amniotic fluid and fetuses, but not in control dam plasma (GD 18). Detection of MEHP in control animal samples might have resulted from sample contamination during collection or analysis, due to the presence of phthalates in manufactured laboratory plasticware. Although not assessed in the present study, DEHP and MEHP can be transferred from dam to offspring via lactation. ${ }^{54}$ DEHP exposure during the 
perinatal period was associated with significantly decreased total and live litter size, due to a significantly decreased number of female pups per litter in the $10,000 \mathrm{ppm}$ group $(626 \mathrm{mg} / \mathrm{kg} /$ day $)$. In previous studies, increased resorptions, postimplantation loss, and whole-litter loss have been observed following DEHP exposure at doses $>500 \mathrm{mg} / \mathrm{kg} / \mathrm{day}$ in pregnant rats. ${ }^{90 ; 207-209}$ In a multigenerational reproductive assessment of DEHP, previously conducted by NTP, significant effects on litter size and sex ratio were observed following perinatal exposure of Sprague Dawley rat $\mathrm{F}_{0}$ dams at concentrations of 7,500 and 10,000 ppm. ${ }^{210}$ In this perinatal and postweaning study (Study 1), exposure-related decreases in birth and weaning mean body weights were observed in both male and female groups. Gestational DEHP exposure was associated with 15\% and 12\% decreases in postnatal day (PND) 1 mean body weights of 10,000 ppm male and female pups, respectively. Further growth retardation during lactation was observed with male and female pup weights. Postweaning, mean body weights of the $10,000 \mathrm{ppm}$ offspring remained significantly decreased relative to control groups throughout the 2-year exposure period. The magnitude of effect on body weight observed in 10,000 ppm offspring was higher than the $30 \%$ decrease in postweaning body weights observed at the same dose level in the NTP multigeneration assessment of DEHP. ${ }^{210}$

No significant differences in overall survival were observed in either the perinatal and postweaning study (Study 1) or the postweaning-only study (Study 2) relative to concurrent control groups, although there was some early postweaning mortality (Study 1). Lower mean body weights (postweaning to study termination) were observed in 10,000 ppm male and female rats in both studies relative to control rats. In both studies, lower mean body weights were associated with lower body weight gain; however, the magnitude of effect was higher following perinatal and postweaning exposure compared to postweaning-only exposure, due to early life exposure that included gestation, lactation, and a brief period after weaning. In the 10,000 ppm male and female rat groups, the largest difference in feed consumption relative to the control groups occurred directly following weaning. In rats, increased rates of feed and water consumption, relative to body weight, are commonly observed in younger animals and decrease with subsequent growth and development. Furthermore, in the perinatal and postweaning study (Study 1), the 2-year direct exposure period began at weaning, 3 weeks earlier than in the postweaning-only study (PND 42) (Study 2). Although this 3-week interval represents a small fraction of the total exposure timeframe, perinatal and postweaning exposure groups were exposed to DEHP at earlier ages and therefore at higher doses than the corresponding groups in the postweaning-only study (Study 2), likely contributing to the 3\%-20\% higher mean chemical consumption (mg/kg/day) postweaning in Study 1 versus Study 2.

The following conclusions on the carcinogenicity of DEHP were determined based on the weight-of-evidence approach described in the Explanation of Levels of Evidence of Carcinogenic Activity. Conclusions on DEHP carcinogenic activity are described separately for the perinatal and postweaning and postweaning-only studies, based on the independent results observed in each study. Although some variability in carcinogenic outcomes was observed between rats exposed to DEHP during the perinatal and postweaning periods and those only exposed postweaning, it is unclear whether any differences correspond to specific developmental mechanisms during the perinatal period. Some noncarcinogenic outcomes were related to perinatal exposure, however, which included the gross lesions in the reproductive tract and histological lesions in the kidney. 
At the conclusion of both studies, numerous neoplastic and nonneoplastic lesions in the liver were identified. In male rats, increased incidences of hepatocellular adenoma and hepatocellular adenoma or carcinoma (combined) were observed in both 2-year studies. In the perinatal and postweaning study (Study 1), there was an increase in rare hepatocellular carcinomas (historical control $2 / 489$; range $0 \%$ to $2 \%$ ) in the 10,000 ppm group $(8.7 \%)$, whereas a positive trend was observed in these neoplasms in the postweaning-only study (Study 2). In both perinatal and postweaning and postweaning-only studies, higher incidences of hepatocellular cytoplasmic alteration, liver pigmentation, and liver necrosis were observed in male rats. Although considered minimal in severity, a higher incidence of hepatocellular hypertrophy was observed in 10,000 ppm male rats exposed during the perinatal and postweaning periods $(35 \%)$ compared to male rats in the postweaning-only study (12\%). Additionally, a significantly increased incidence of basophilic focus was observed in the livers of 10,000 ppm male rats in the perinatal and postweaning study, but not in their counterparts in the postweaning-only study (Study 2). Taken together, the significantly increased incidence of hepatocellular adenoma or carcinoma (combined) supported clear evidence of carcinogenicity in male rats in both 2-year studies.

In female rats, increased incidences of hepatocellular adenoma, hepatocellular carcinoma, and hepatocellular adenoma or carcinoma (combined) were observed in both 2-year studies. In female rats in the perinatal and postweaning study, the incidence of hepatocellular adenomas in the 3,000 ppm group that was higher (18\%) than in the historical control range $(15 / 489$; range $0 \%$ to $8 \%$ ); in females in the postweaning-only study (Study 2), the incidence of hepatocellular adenomas was above the historical control range in the 10,000 ppm group. Furthermore, an increased incidence of hepatocellular carcinoma (17\%), a rare neoplasm type (historical control $1 / 489$; range $0 \%$ to $2 \%$ ), was also observed in $10,000 \mathrm{ppm}$ female rats in the perinatal and postweaning study, whereas there was occurrence of hepatocellular carcinomas $(0 \%$ versus $4 \%$ compared to the control group) in the 10,000 ppm group of the postweaning-only study (Study 2). Taken together, the increased incidence of hepatocellular adenoma or carcinoma (combined) supported clear evidence of carcinogenicity in the liver of female rats in both studies.

Significantly increased incidences of hepatocellular cytoplasmic alteration, hepatocellular hypertrophy, and liver pigmentation were observed in female rats in both 2-year studies. Higher incidences of eosinophilic foci were observed in 10,000 ppm female rats in the perinatal and postweaning study (Study 1), but not in the postweaning-only study (Study 2). A significantly increased incidence of hepatocellular carcinomas was observed only in $10,000 \mathrm{ppm}$ females of the perinatal and postweaning study $(17 \%)$ compared to the postweaning-only study $(4 \%)$. This observation could be due to a higher rate of progression from hepatocellular adenoma to carcinoma because of early life or prolonged exposure, observations that are similar to those made in the perfluorooctanoic acid (PFOA) 2-year study ${ }^{211}$ in which perinatal and postweaning exposure led to a marginally higher carcinoma rate $(4 \%)$ of this rare neoplasm in male rats relative to the rats with postweaning-only exposure $(0 \%)$. Males with perinatal and postweaning exposure to DEHP also had a marginally higher hepatocellular carcinoma incidence compared to males with postweaning-only exposures.

Numerous chronic exposure studies have found that DEHP induces hepatic neoplasms in rats and mice. In the current study, estimated DEHP daily exposure concentrations ( $\mathrm{mg} / \mathrm{kg} / \mathrm{day}$ )

associated with higher incidences of hepatocellular adenomas or carcinomas were comparable to concentrations reported in previous studies. In a previous NTP study, chronic DEHP exposure via dosed feed resulted in increased incidences of hepatocellular carcinomas in male and female 
Fischer 344 (F344) rats at estimated daily exposures of 322 and $674 \mathrm{mg} / \mathrm{kg} /$ day in males and 394 and $774 \mathrm{mg} / \mathrm{kg} /$ day in females. ${ }^{69}$ In another study, increased incidences of hepatocellular adenomas and carcinomas were observed in male Sprague Dawley rats following lifetime exposure to $300 \mathrm{mg} / \mathrm{kg} /$ day. ${ }^{126}$ The precise mechanism by which DEHP induces hepatic neoplasms is not fully characterized. However, activation of peroxisome proliferator-activated receptor alpha (PPAR $\alpha$ ) by the DEHP proximal metabolite MEHP has been defined as a key molecular event by which DEHP causes hepatic neoplasms in rodents. The human relevance of mechanisms of carcinogenesis of the peroxisome proliferator class of chemicals is frequently debated. ${ }^{212}$ Additional research suggests that multiple signaling pathways and downstream mediators likely contribute to DEHP-induced hepatic carcinogenesis, rather than a single hallmark event such as activation of PPAR. ${ }^{73 ; 212}$

Increased incidences of pancreatic acinar adenomas, carcinomas, and adenoma or carcinoma (combined) were observed in male rats in both studies. In both perinatal and postweaning and postweaning-only exposed male rats, increased incidences of pancreatic acinar adenomas occurred in the 3,000 and 10,000 ppm groups at higher rates than the historical control range $(60 / 488$; range $0 \%$ to $28 \%)$. Notably, a higher incidence of pancreatic cell adenomas occurred in 3,000 ppm perinatal and postweaning-exposed males (72\%) when compared to males exposed to the same concentration postweaning-only (46\%). Due to the potentially high background incidence of pancreatic acinar adenoma in the test rat strain (up to $28 \%$ in historical control groups), observed differences between perinatal and postweaning and postweaning-only exposure groups might have resulted from background variability of this lesion and not increased sensitivity related to perinatal exposure. A higher incidence of pancreatic acinar carcinoma, a rare neoplasm type (historical control 4/488; range $0 \%$ to $4 \%$ ), was observed in 3,000 ppm male rats $(6 \%)$ exposed during the perinatal and postweaning periods, and this neoplasm was increased in the 10,000 ppm male rats exposed postweaning-only. Furthermore, increased incidences of pancreatic acinar hyperplasia were noted in 3,000 and 10,000 ppm males exposed postweaning-only. The increased incidence of pancreatic acinar adenoma or carcinoma (combined) was considered clear evidence of carcinogenicity in male rats in both 2-year studies.

In female rats, occurrences of pancreatic acinar adenoma and carcinomas were observed in the postweaning-only study (Study 2), whereas occurrences of pancreatic acinar adenomas were observed in the perinatal and postweaning study (Study 1). In contrast to males, pancreatic acinar neoplasms are very rare in female rats (historic control 0/489). Occurrences of pancreatic acinus hyperplasia were also observed in exposed groups in both studies. After considering the rarity of this lesion type in female rats, the corroborating effect in male rats, and findings supportive of neoplastic progression, the incidence of pancreatic acinar adenoma or carcinoma (combined) was considered related to DEHP exposure in female rats.

Pancreatic acinar adenomas and carcinomas have been observed previously in male F344 rats following chronic DEHP exposure. ${ }^{86}$ Pancreatic adenomas have been reported in rodent models following exposure to various chemicals known to activate PPAR $\alpha$, such as PFOA, butyl benzyl phthalate (BBP), and Wyeth-14,643 (WY). ${ }^{211 ; 213 ; 214}$ Although direct activity of PPAR $\alpha$ agonists on acinar cells has yet to be established, some data suggest that induction of pancreatic neoplasms by PPAR $\alpha$ agonists is secondary to functional alterations in the liver. One proposed mode of action suggests that hepatic PPAR $\alpha$ activation and subsequent alteration of transcriptional activity leads to alteration in bile acid composition and flow, resulting in 
cholestasis and increased expression of cholecystokinin (CCK). ${ }^{127} \mathrm{CCK}$ is a growth factor reported to induce normal, adaptive, and neoplastic growth of pancreatic acinar cells in rats. ${ }^{215-217}$

Numerous gross lesions in the male reproductive tract were observed in male rats in the 10,000 ppm group exposed during both the perinatal and postweaning period, consistent with the "phthalate syndrome" suite of effects. ${ }^{9 ; 218 ; 219}$ These findings included decreased size of the phallus, testes, epididymides, prostate, seminal vesicles, and levator ani/bulbocavernosus (LABC) muscle; gubernacular length exceeding $20 \mathrm{~mm}$; no gubernaculum present; nonregression of the cranial suspensory ligament (CSL); cleft phallus or prepuce; undescended testes (cryptorchid); epididymal agenesis (caput, corpus, or cauda); and incomplete preputial separation. All examined males exposed to $10,000 \mathrm{ppm}$ DEHP in the perinatal and postweaning study presented with at least one of the aforementioned reproductive tract malformations; small or undescended testes were the most frequently observed reproductive tract malformations at $10,000 \mathrm{ppm}$. Male reproductive tract malformations have been observed in rodents following perinatal exposure to various phthalates, such as DEHP, di- $n$-butyl phthalate (DBP), di-isobutyl phthalate (DiBP), BBP, and diisononyl phthalate (DINP), and are indicative of hormone disruption of developmental androgen and insulin-like peptide 3 (Ins13) dependent signaling pathways. ${ }^{95 ; 220 ; 221}$ Differentiation of Wolffian structures (e.g., the epididymis, vas deferens, seminal vesicles) depends on fetal testosterone signaling, and masculinization of the prostate and external genitalia depends on the biosynthesis and signaling of the more potent androgen, dihydrotestosterone. ${ }^{222}$ Targeted disruption of Ins13 signaling alters gubernaculum development and CSL regression, leading to cryptorchidism. ${ }^{223 ; 224}$ Developmental exposure to phthalates, such as DEHP, DBP, and BBP, disrupts Insl3 signaling leading to complete agenesis or hypoplasia of the gubernacular ligaments and retention of the testes in the inguinal or abdominal position. ${ }^{95 ; 225}$ In the study presented here, undescended testes were consistently reduced in size and were more often retained in the abdominal region compared to the inguinal region.

Additional microscopic nonneoplastic lesions diagnosed in the testis and epididymis of male rats exposed during both the perinatal and postweaning periods were considered related to DEHP exposure. Observations of testicular germinal epithelium degeneration were noted in 10,000 ppm male rats with or without perinatal exposure and occurred concomitantly with epididymal hypospermia. Findings of testicular edema and exfoliated germ cells in the epididymal duct were observed in 10,000 ppm males of both studies; however, these effects were not significant in the perinatal and postweaning study and therefore considered related to exposure only in the postweaning-only study (Study 2). It is possible that evaluation at the end of a 2-year exposure led to lower sensitivity in detecting testicular degeneration as a higher incidence in control males can occur in older animals and findings are likely to be more evident in younger animals at lower exposure concentrations at earlier timepoints than in younger control animals. ${ }^{226 ; 227}$ Seminiferous tubule dysgenesis was only present in $10,000 \mathrm{ppm}$ males with perinatal and postweaning exposure (10/49). Seminiferous tubule dysgenesis is characterized as a developmental malformation seen microscopically as aberrant or misshapen seminiferous tubules, either with no or dilated lumens, which are often surrounded by focal Leydig cell aggregates. The Leydig cell aggregates within foci of dysgenesis differ morphologically from the Leydig cells in adenomas. The Leydig cells in these foci of dysgenesis appear to be poorly differentiated, are spindle-shaped and resemble embryonic Leydig cells, and do not have the abundant eosinophilic or vacuolated cytoplasm often apparent in hyperplasia or adenoma. ${ }^{226}$ Dysgenetic lesions might occur as one or more small foci per testis, and tend to be located near 
the center of the testis or might occupy the entire testis. ${ }^{226}$ The malformed tubules can appear to form anastomotic networks. The dysgenetic tubules contain poorly differentiated Sertoli cells, with small, elongated, and sometimes cleaved nuclei and less prominent nucleoli than the typical, prominent, tripartite nucleoli seen in mature Sertoli cells. Spermatogenesis is absent in these foci of dysgenesis but can be present elsewhere in the testis. Dysgenetic foci might be present in one or both testes and can be more severe in undescended than in scrotal testes. ${ }^{228 ;}{ }^{229}$ In men, similar microscopic dysgenetic foci have been reported in cryptorchid (undescended) testes, ${ }^{230}$ in testes also containing testicular cancer (both scrotal and cryptorchid testes ${ }^{231}$ ), and from testicular biopsies from the contralateral testis in men undergoing orchiectomy for testicular cancer. ${ }^{232}$

Increased pituitary pars distalis hypertrophy occurred in 10,000 ppm male rats in both studies. This lesion is commonly associated with disruption of the hypothalamus-pituitary-gonad signaling axis. Loss of negative feedback signaling by testicular-derived androgens, due to the antiandrogenic activity of phthalates, leads to increased hypothalamic release of gonadotropinreleasing hormone and subsequent increased releases of luteinizing hormone and folliclestimulating hormone by gonadotrophs, or "castration cells," in the pars distalis of the pituitary gland. ${ }^{233}$

There was a significant positive trend with testicular interstitial cell adenoma neoplasms in the postweaning-only study (Study 2), and the incidence of testicular interstitial cell adenoma $(15 / 50 ; 30 \%)$ observed in 10,000 ppm male rats was above NTP's historical control range (19/487; range $0 \%$ to $14 \%)$. However, there were no significant pairwise differences among the exposed groups compared to the control groups in the incidences of neoplasms or hyperplasias. Taken together, the data suggest that testicular interstitial cell adenomas may have been related to DEHP exposure in postweaning-only exposed male rats.

In contrast, perinatal and postweaning exposure did not increase the incidence of Leydig cell (interstitial) neoplasms relative to control animals, although the incidence of interstitial cell hyperplasia was considerably higher. Currently, it is unclear whether developmental malformations in the male reproductive tract, such as altered seminiferous tubule morphology or structural and functional alterations in Sertoli and Leydig cell populations, affect the carcinogenic potential of DEHP in testes in perinatally exposed rats relative to functional effects observed following adult exposure only. Increased incidences of focal interstitial cell hyperplasia were observed in both 2-year studies. Focal hyperplasia is considered a preneoplastic lesion that commonly forms as a part of a continuum leading to interstitial cell adenoma; it is distinct from diffuse hyperplasia, generally considered a physiological response to hormonal imbalance. ${ }^{234-236}$

Several PPAR $\alpha$ agonists, including DEHP, PFOA, and WY, have been shown to induce Leydig cell neoplasms in rats. ${ }^{126 ; 214}$ Multiple mechanisms by which PPAR $\alpha$ agonists might induce testicular neoplasms through disruption of the hypothalamus-pituitary-thyroid axis have been postulated; however, the weight of evidence is currently inadequate to establish a mode of action. ${ }^{127}$ The marginal to no response in Leydig cell neoplasms to DEHP in this study is inconsistent with published studies and could be due to differential diagnoses. Varying morphological criteria distinguishing Leydig cell adenomas from seminiferous tubule dysgenesis may account for differential diagnoses; ${ }^{226}$ the rodent strain studies may also be a factor as there were no Leydig cell neoplasms observed in the NTP PFOA studies, ${ }^{211}$ which used the Hsd:Sprague Dawley ${ }^{\circledR}$ SD $^{\circledR}$ rat. 
In female rats, increased incidences of adenoma, adenocarcinoma, squamous cell carcinoma, or squamous cell papilloma (combined) (mostly adenocarcinoma) were observed in the uterus (including cervix). Higher incidences of uterine (including cervix) adenoma, adenocarcinoma, squamous cell carcinoma, or squamous cell papilloma (combined) $(26 \%)$ were observed in 10,000 ppm postweaning-only exposed females, which was above the NTP historical control incidence of this combination of lesions of $2 \%$ to $10 \%$. Uterine inflammation was increased in all DEHP-exposed groups in the postweaning-only study (Study 2). This supported clear evidence of carcinogenic activity from DEHP postweaning-only exposure in female rats.

In the perinatal and postweaning study (Study 1), however, there was a marginally higher incidence of uterine neoplasms in DEHP-exposed groups compared to the control group and none of the pairwise comparisons to the control group were significant. The magnitude of the difference between the 10,000 ppm group and the control group in each study was lower in the perinatal and postweaning study ( $8 \%$ difference) compared to the postweaning-only study ( $22 \%$ difference). There was reduced certainty in this marginal response such that the incidence of uterine neoplasms may have been related to perinatal and postweaning exposure. The reason for this is not clear, but it is noteworthy that the testis and uterus, sites of endocrine action, had a lower response in general with the perinatal and postweaning exposure compared to the postweaning-only exposure.

The present study is the first to identify an association between DEHP exposure and induction of uterine neoplasms in female rats. The mechanism for this response is unclear. For previous chronic studies, no alterations in female reproductive organ histology were reported. ${ }^{69 ; 86 ; 123}$ Induction of the "tumor triad," including liver, Leydig cell, and pancreatic acinar cell tumors, is a finding characteristic of sustained exposure to PPAR $\alpha$ agonists in rats; ${ }^{214}$ however, the relevance of this finding to humans is uncertain. The uterine neoplasm response in the NTP PFOA carcinogenicity study ${ }^{211}$ was considered equivocal evidence of carcinogenic activity. The magnitude of the response and level of evidence for PFOA is similar to that of the DEHP perinatal and postweaning study, whereas the response in the DEHP postweaning-only study was considerably higher. Further work will be required to assess the mode of action for these outcomes.

Evidence of DEHP-associated renal toxicity was specific to male and female rats with perinatal and postweaning exposure. Numerous nonneoplastic kidney lesions were increased in DEHP-exposed groups relative to control groups, such as papilla edema, papilla epithelium hyperplasia, papilla hemorrhage, infarct, and renal tubule cysts. Papillary edema was the most prevalent kidney lesion in 10,000 ppm male (39/49) and female (38/48) rats in the perinatal and postweaning study and was not present in the postweaning-only study (Study 2). This highly unusual bilateral lesion was characterized by marked dilation and/or distortion of the collecting ducts and moderate to marked expansion of the papilla interstitium by pale eosinophilic to fibrillary amphophilic material, consistent with edema. Periodic acid-Schiff (PAS) staining demonstrated that the basement of vascular structures, in addition to the renal tubule basement membranes in the cortex and medulla, were intact. An abrupt loss of PAS staining of the basement membranes of collecting tubules was observed, however, at the junction of the outer and inner medulla. Therefore, perinatal exposure to DEHP is presumed to interfere directly or indirectly with the proper development of the collecting tubules. The normal function of the collecting duct system is urine transport as well as electrolyte and fluid balance through reabsorption and excretion, processes regulated by aldosterone and vasopressin. Additional 
studies have reported DEHP-associated renal toxicity. Chronic dietary exposure to DEHP $(\geq 789 \mathrm{mg} / \mathrm{kg} /$ day) was associated with increased severity of routinely occurring renal tubule pigmentation and chronic progressive nephropathy in male and female rats. ${ }^{86}$ Altered kidney function and kidney lesions have been reported in rats following developmental DEHP exposure. Impaired kidney development and function were observed in adult Wistar rats following maternal exposure to DEHP at doses of 0.25 and $6.25 \mathrm{mg} / \mathrm{kg}$ /day from GD 0 through offspring PND 21. ${ }^{237}$ Maternal exposure resulted in a decreased number of nephrons, higher glomerular volume, and smaller Bowman's capsule in offspring at weaning, as well as glomerulosclerosis, interstitial fibrosis, and effacement of podocyte foot processes in 33-week-old $F_{1}$ rats. Taken together, these data suggest the developing kidney may be a sensitive target of DEHP toxicity.

Cardiovascular findings of increased heart valve fibrosis and thrombus were present in 10,000 ppm male rats in both of the present studies. Thrombosis in male rats has been associated with pancreatic cancer related to onset of an intrinsic hypercoagulable state caused by elevated activation of platelets and increased expression of procoagulant factors. ${ }^{238}$ However, a low concurrence of pancreatic cancer and cardiovascular thrombosis was observed in the present studies. Additionally, increased systolic blood pressure has been observed in rats and mice exposed to DEHP; however, this effect is thought to be secondary to renal dysfunction or alterations in renin and angiotensin II signaling. ${ }^{237 ;} 239$

NTP has tested DEHP in a range of in vitro and in vivo genotoxicity assays, and the results were generally negative. The positive results seen in some of the in vitro assays for induction of sister chromatid exchanges (SCE) were seen in the presence of excessive cytotoxicity. The

Organization for Economic Co-operation and Development test guideline ${ }^{240}$ for the in vitro SCE test was withdrawn in 2014, and the test is no longer requested by regulatory agencies. In vivo, the nonnegative responses that were observed in some of the NTP micronucleus assays were generally weak. The consensus from published data is that DEHP shows limited evidence of genotoxic potential, and for the sporadic positive results that have been reported, the response is either weak, not reproducible, obtained in a nonstandard test system, or qualified to some degree by the authors.

Lastly, carcinogenic responses that were related or may have been related to DEHP exposure were modeled to estimate benchmark doses corresponding to a $10 \%$ increase in neoplasm incidence $\left(\mathrm{BMD}_{10}\right)$. For the similar target sites, the $\mathrm{BMD}_{10}$ levels based on the hepatocellular response were lower in males in the perinatal and postweaning study compared to the postweaning-only study (383 mg/kg/day and $434 \mathrm{mg} / \mathrm{kg} /$ day, respectively) and in females (123 mg/kg/day and $384 \mathrm{mg} / \mathrm{kg} /$ day, respectively). Conversely, BMD 10 levels were lower for the pancreatic acinar neoplasm response in males with postweaning-only exposure compared with perinatal and postweaning exposure $(31 \mathrm{mg} / \mathrm{kg} /$ day versus $86 \mathrm{mg} / \mathrm{kg} /$ day, respectively). The $\mathrm{BMD}_{10}$ for the neoplastic responses in the uterus in females was lower in the postweaning-only study compared to the perinatal and postweaning study $(324 \mathrm{mg} / \mathrm{kg} /$ day versus $594 \mathrm{mg} / \mathrm{kg} / \mathrm{day}$, respectively). The lowest $\mathrm{BMD}_{10}$ levels were associated with incidences of pancreatic acinar adenoma or carcinoma (combined) in male rats in both studies. These data show no obvious overall increased sensitivity in carcinogenic response with perinatal and postweaning exposure compared to postweaning-only exposure, which is consistent with NTP's study of PFOA perinatal exposure. ${ }^{211}$ 


\section{Conclusions}

Under the conditions of the perinatal and postweaning feed study (Study 1), there was clear evidence of carcinogenic activity of di(2-ethylhexyl) phthalate (DEHP) in male Hsd:Sprague Dawley ${ }^{\circledR} \mathrm{SD}^{\circledR}$ rats based on the increased incidences of hepatocellular adenoma or carcinoma (combined) and acinar adenoma or carcinoma (combined) neoplasms (predominately adenomas) of the pancreas. There was clear evidence of carcinogenic activity of DEHP in female Hsd:Sprague Dawley ${ }^{\circledR} \mathrm{SD}^{\circledR}$ rats based on the increased incidence of hepatocellular adenoma or carcinoma (combined). The occurrence of pancreatic acinar adenoma or carcinoma (combined) was considered to be related to exposure. The occurrence of uterine (including cervix) adenoma, adenocarcinoma, squamous cell carcinoma, or squamous cell papilloma (combined) in female rats may have been related to exposure.

Under the conditions of the postweaning-only feed study (Study 2), there was clear evidence of carcinogenic activity of DEHP in male Hsd:Sprague Dawley ${ }^{\circledR} \mathrm{SD}^{\circledR}$ rats based on the increased incidences of hepatocellular adenoma or carcinoma (combined) and acinar adenoma or carcinoma (combined) neoplasms (predominately adenomas) of the pancreas. The occurrence of testicular interstitial cell adenoma in male rats may have been related to exposure. There was clear evidence of carcinogenic activity of DEHP in female Hsd:Sprague Dawley ${ }^{\circledR} \mathrm{SD}^{\circledR}$ rats based on the increased incidences of hepatocellular adenoma or carcinoma (combined) and uterine (including cervix) adenoma, adenocarcinoma, squamous cell carcinoma, or squamous cell papilloma (combined). The occurrence of pancreatic acinar adenoma or carcinoma (combined) in female rats was considered to be related to exposure.

The BMD analysis shows there was no consistent pattern indicating that perinatal and postweaning exposure was more sensitive compared to postweaning-only exposure and modeled responses were within threefold of each other. However, there was a stronger carcinogenic response in the reproductive organs (uterus and testis) in the postweaning-only exposure study compared to the perinatal and postweaning exposure study.

Perinatal and postweaning exposure to DEHP (Study 1) resulted in increased incidence of nonneoplastic lesions in the liver, kidney, heart (male), pancreas (female), pituitary gland (male), bone marrow (male), testis, and epididymis. In addition, exposure increased gross lesions within the reproductive tract of males and females.

Postweaning exposure to DEHP (Study 2) resulted in increased incidence of nonneoplastic lesions in the liver, pancreas, heart (male), pituitary gland (male), bone marrow (male), testis, epididymis, and uterus. 


\section{References}

1. National Center for Biotechnology Information (NCBI). PubChem Compound Summary: Bis(2-ethylhexyl) phthalate, CID 8343. Bethesda, MD: National Center for Biotechnology Information. https://pubchem.ncbi.nlm.nih.gov/compound/Bis 2-ethylhexyl_phthalate

2. National Institute for Occupational Safety and Health (NIOSH). NIOSH pocket guide to chemical hazards, di-sec octyl phthalate. U.S. Department of Health and Human Services, Centers for Disease Control and Prevention, National Institute for Occupational Safety and Health; 2019. https://www.cdc.gov/niosh/npg/npgd0236.html

3. U.S. Environmental Protection Agency (USEPA). CompTox Chemistry Dashboard: Di(2ethylhexyl) phthalate 117-81-7 | DTXSID5020607.

https:/comptox.epa.gov/dashboard/DTXSID5020607

4. Agency for Toxic Substances and Disease Registry (ATSDR). Toxicological profile for di(2ethylhexyl)phthalate (DEHP). Atlanta, GA: U.S. Department of Health and Human Services, Public Health Service; 2019.

5. U.S. Environmental Protection Agency (USEPA). Chemical data reporting (2012 and 2016 public CDR database). Washington, DC. : U.S. Environmental Protection Agency, Office of Pollution Prevention and Toxics; 2017.

6. Toxics Use Reduction Institute (TURI). Five chemicals alternatives reduction study. Final report. Chapter 7. DEHP. Lowell, MA: The Massachusetts Toxics Use Reduction Institute, University of Massachusetts Lowell; 2005.

https://www.turi.org/TURI_Publications/TURI_Methods_Policy_Reports/Five_Chemicals_Alter natives Assessment Study. 2006

7. European Commission (EC). Bis(2-ethylhexyl)phthalate (DEHP) - Summary risk assessment report Ispra (VA), Italy: European Chemicals Bureau, Institute for Health and Consumer Protection, Toxicology and Chemical Substance (TCS); 2008. EUR 23384EN/2.

8. Latini G, Ferri M, Chiellini F. Materials degradation in PVC medical devices, DEHP leaching and neonatal outcomes. Curr Med Chem. 2010; 17(26):2979-2989.

https://doi.org/10.2174/092986710792064992

9. Bouattour Y, Wasiak M, Bernard L, Pinguet J, Richard D, Le Rouzo-Grèves M, Dhifallah I, Lambert C, Pereira B, Chennell P et al. Quantification of bis(2-ethylhexyl) phthalate released by medical devices during respiratory assistance and estimation of patient exposure. Chemosphere. 2020; 255:126978. https://doi.org/10.1016/i.chemosphere.2020.126978

10. International Programme on Chemical Safety (IPCS). Environmental Health Criteria 131: Diethylhexyl phthalate. 1992.

11. Serrano SE, Braun J, Trasande L, Dills R, Sathyanarayana S. Phthalates and diet: A review of the food monitoring and epidemiology data. Environ Health. 2014; 13(1):43-43.

https://doi.org/10.1186/1476-069X-13-43 
12. Clark K, Cousins, I. T. and Mackay, D. Assessment of critical exposure pathways. In: Staples CA, editor. The Handbook of Environmental Chemistry. New York: Springer-Verlag; 2003. p. 227-262.

13. National Toxicology Program (NTP). NTP-CERHR monograph on the potential human reproductive and developmental effects of di (2-ethylhexyl) phthalate (DEHP). Research Triangle Park, NC: U.S. Department of Health and Human Services, National Institute of Environmental Health Sciences, National Toxicology Program; 2006.

14. Fromme H, Lahrz T, Piloty M, Gebhart H, Oddoy A, Ruden H. Occurrence of phthalates and musk fragrances in indoor air and dust from apartments and kindergartens in Berlin (Germany). Indoor Air. 2004; 14(3):188-195. https://doi.org/10.1111/j.1600-0668.2004.00223.x

15. Centers for Disease Control and Prevention (CDC). Fourth national report on human exposure to environmental chemicals. Updated tables, January 2019, volume one. Atlanta, GA: U.S. Department of Health and Human Services, Centers for Disease Control and Prevention; 2019.

https://www.cdc.gov/exposurereport/pdf/FourthReport UpdatedTables Volume1 Jan2019508.pdf

16. Wambaugh JF, Wang A, Dionisio KL, Frame A, Egeghy P, Judson R, Setzer RW. High throughput heuristics for prioritizing human exposure to environmental chemicals. Environ Sci Technol. 2014; 48(21):12760-12767. https://doi.org/10.1021/es503583j

17. Dirven HA, van den Broek PH, Arends AM, Nordkamp HH, de Lepper AJ, Henderson PT, Jongeneelen FJ. Metabolites of the plasticizer di(2-ethylhexyl)phthalate in urine samples of workers in polyvinylchloride processing industries. Int Arch Occup Environ Health. 1993; 64(8):549-554. https://doi.org/10.1007/bf00517699

18. Nielsen J, Akesson B, Skerfving S. Phthalate ester exposure--air levels and health of workers processing polyvinylchloride. Am Ind Hyg Assoc J. 1985; 46(11):643-647.

https://doi.org/10.1080/15298668591395463

19. Hines CJ, Hopf NBN, Deddens JA, Silva MJ, Calafat AM. Estimated daily intake of phthalates in occupationally exposed groups. J Expo Sci Environ Epidemiol. 2011; 21(2):133141. https://doi.org/10.1038/jes.2009.62

20. Hines CJ, Nilsen Hopf NB, Deddens JA, Calafat AM, Silva MJ, Grote AA, Sammons DL. Urinary phthalate metabolite concentrations among workers in selected industries: A pilot biomonitoring study. Ann Occup Hyg. 2009; 53(1):1-17. https://doi.org/10.1093/annhyg/men066

21. Fong JP, Lee FJ, Lu IS, Uang SN, Lee CC. Estimating the contribution of inhalation exposure to di-2-ethylhexyl phthalate (DEHP) for PVC production workers, using personal air sampling and urinary metabolite monitoring. Int J Hyg Environ Health. 2014; 217(1):102-109. https://doi.org/10.1016/j.ijheh.2013.04.002

22. Wang W, Xu X, Fan CQ. Health hazard assessment of occupationally di-(2-ethylhexyl)phthalate-exposed workers in China. Chemosphere. 2015; 120:37-44.

https://doi.org/10.1016/j.chemosphere.2014.05.053 
23. Ginsberg G, Ginsberg J, Foos B. Approaches to children's exposure assessment: Case study with diethylhexylphthalate (DEHP). Int J Environ Res Public Health. 2016; 13(7). https://doi.org/10.3390/ijerph13070670

24. Zhu J, Phillips SP, Feng YL, Yang X. Phthalate esters in human milk: Concentration variations over a 6-month postpartum time. Environ Sci Technol. 2006; 40(17):5276-5281. https://doi.org/10.1021/es060356w

25. Swedish National Chemicals Inspectorate (KEMI). Risk Assessment - bis(2-ethylhexyl) phthalate (CAS-No.: 117-81-7). 2000.

26. Silva MJ, Reidy JA, Herbert AR, Preau JL, Jr., Needham LL, Calafat AM. Detection of phthalate metabolites in human amniotic fluid. Bull Environ Contam Toxicol. 2004; 72(6):12261231. https://doi.org/10.1007/s00128-004-0374-4

27. U.S. Food and Drug Administration (FDA). Safety assessment of di(2-ethylhexyl)phthalate (DEHP) released from PVC medical devices. Rockville, MD; 2001.

28. Den Braver-Sewradj SP, Piersma A, Hessel EVS. An update on the hazard of and exposure to diethyl hexyl phthalate (DEHP) alternatives used in medical devices. Crit Rev Toxicol. 2020; 50(8):650-672. https://doi.org/10.1080/10408444.2020.1816896

29. Tickner JA, Schettler T, Guidotti T, McCally M, Rossi M. Health risks posed by use of Di-2ethylhexyl phthalate (DEHP) in PVC medical devices: A critical review. Am J Ind Med. 2001; 39(1):100-111. https://doi.org/10.1002/1097-0274(200101)39:1<100::aid-ajim10>3.0.co;2-q

30. 110th U.S. Congress. Consumer Product Safety Improvement Act of 2008. 2008.

31. Code of Federal Regulations (CFR). 2017; 17(Part 1307).

32. Code of Federal Regulations (CFR). 2001; 21(Part 177, Subpart B, Section 177.1010).

33. Code of Federal Regulations (CFR). 2001; 21(Part 177, Subpart B, Section 177.1200).

34. Carpenter CP, Weil CS, Smyth HF, Jr. Chronic oral toxicity of di-(2-ethylhexyl) phthalate of rats, guinea pigs, and dogs. AMA Arch Ind Hyg Occup Med. 1953; 8(3):219-226.

35. U.S. Environmental Protection Agency (USEPA). Integrated Risk Information System (IRIS), chemical assessment summary, di(2-ethylhexyl)phthalate; CASRN 117-81-7. Washington D.C.: U.S. Environmental Protection Agency, National Center for Environmental Assessment; 1987.

https://cfpub.epa.gov/ncea/iris/iris_documents/documents/subst/0014_summary.pdf\#nameddest= $\underline{\mathrm{rfd}}$

36. U.S. Environmental Protection Agency (USEPA). National Primary Drinking Water Regulations: Phthalate, di(2-Ethylhexyl). Washington D.C.: Office of Water; 1995.

37. Occupational Safety and Health Administration (OSHA). Recommendations for consideration by the U.S. Secretary of Labor on the Adoption and use of occupational exposure limits by federal agencies. Occupational Safety and Health Administration, Federal Advisory 
Council on Occupational Safety and Health; 2012.

https://www.osha.gov/dep/facosh/Exhibit 9b.pdf

38. Lake BG, Gray TJ, Foster JR, Stubberfield CR, Gangolli SD. Comparative studies on di-(2ethylhexyl) phthalate-induced hepatic peroxisome proliferation in the rat and hamster. Toxicol Appl Pharmacol. 1984; 72(1):46-60. https://doi.org/10.1016/0041-008x(84)90248-5

39. Albro PW. Absorption, metabolism, and excretion of di(2-ethylhexyl) phthalate by rats and mice. Environ Health Perspect. 1986; 65:293-298. https://doi.org/10.1289/ehp.8665293

40. Albro PW, Corbett JT, Schroeder JL, Jordan S, Matthews HB. Pharmacokinetics, interactions with macromolecules and species differences in metabolism of DEHP. Environ Health Perspect. 1982; 45:19-25. https://doi.org/10.1289/ehp.824519

41. Rhodes C, Orton TC, Pratt IS, Batten PL, Bratt H, Jackson SJ, Elcombe CR. Comparative pharmacokinetics and subacute toxicity of di(2-ethylhexyl) phthalate (DEHP) in rats and marmosets: Extrapolation of effects in rodents to man. Environ Health Perspect. 1986; 65:299307. https://doi.org/10.1289/ehp.8665299

42. Pollack GM, Li RC, Ermer JC, Shen DD. Effects of route of administration and repetitive dosing on the disposition kinetics of di(2-ethylhexyl) phthalate and its mono-de-esterified metabolite in rats. Toxicol Appl Pharmacol. 1985; 79(2):246-256. https://doi.org/10.1016/0041$\underline{008 x(85) 90346-1}$

43. Daniel JW, Bratt H. The absorption, metabolism and tissue distribution of di(2ethylhexyl)phthalate in rats. Toxicology. 1974; 2(1):51-65. https://doi.org/10.1016/0300$\underline{483 \times(74) 90042-0}$

44. Elsisi AE, Carter DE, Sipes IG. Dermal absorption of phthalate diesters in rats. Fundam Appl Toxicol. 1989; 12(1):70-77. https://doi.org/10.1016/0272-0590(89)90063-8

45. Tanaka A, Adachi T, Takahashi T, Yamaha T. Biochemical studies on phthalic esters I. Elimination, distribution and metabolism of di-(2-ethylhexyl)phthalate in rats. Toxicology. 1975; 4(2):253-264. https://doi.org/10.1016/0300-483x(75)90105-5

46. Ikeda GJ, Sapienza PP, Couvillion JL, Farber TM, van Loon EJ. Comparative distribution, excretion and metabolism of di-(2-ethylhexyl) phthalate in rats, dogs and miniature pigs. Food Cosmet Toxicol. 1980; 18(6):637-642. https://doi.org/10.1016/s0015-6264(80)80012-5

47. Chu I, Villeneuve DC, Secours V, Franklin C, Rock G, Viau A. Metabolism and tissue distribution of mono-2-ethylhexyl phthalate in the rat. Drug Metab Dispos. 1978; 6(2):146-149.

48. Lindgren A, Lindquist NG, Lyden A, Olsson T, Ullberg S. A whole body autoradiographic study on the distribution of 14C-labelled di-(2-ethylhexyl)phthalate in mice. Toxicology. 1982; 23(2-3):149-158. https://doi.org/10.1016/0300-483x(82)90094-4

49. Oishi S. Effects of phthalic acid esters on testicular mitochondrial functions in the rat. Arch Toxicol. 1990; 64(2):143-147. https://doi.org/10.1007/bf01974400 
50. Sjöberg P, Bondesson U, Kjellen L, Lindquist NG, Montin G, Ploen L. Kinetics of di-(2ethylhexyl) phthalate in immature and mature rats and effect on testis. Acta Pharmacol Toxicol (Copenh). 1985; 56(1):30-37. https://doi.org/10.1111/j.1600-0773.1985.tb01249.x

51. Teirlynck OA, Belpaire F. Disposition of orally administered di-(2-ethylhexyl) phthalate and mono-(2-ethylhexyl) phthalate in the rat. Arch Toxicol. 1985; 57(4):226-230.

https://doi.org/10.1007/bf00324782

52. Singh AR, Lawrence WH, Autian J. Maternal-fetal transfer of 14C-di-2-ethylhexyl phthalate and 14C-diethyl phthalate in rats. J Pharm Sci. 1975; 64(8):1347-1350.

https://doi.org/10.1002/jps.2600640819

53. Srivastava S, Awasthi VK, Srivastava SP, Seth PK. Biochemical alterations in rat fetal liver following in utero exposure to di(2-ethylhexyl)phthalate (DEHP). Indian J Exp Biol. 1989; 27(10):885-888.

54. Dostal LA, Weaver RP, Schwetz BA. Transfer of di(2-ethylhexyl) phthalate through rat milk and effects on milk composition and the mammary gland. Toxicol Appl Pharmacol. 1987; 91(3):315-325. https://doi.org/10.1016/0041-008x(87)90054-8

55. Sjöberg P, Lindqvist NG, Ploen L. Age-dependent response of the rat testes to di(2ethylhexyl) phthalate. Environ Health Perspect. 1986; 65:237-242.

https://doi.org/10.1289/ehp.65-1474698

56. Egestad B, Green G, Sjöberg P, Klasson-Wehler E, Gustafsson J. Chromatographic fractionation and analysis by mass spectrometry of conjugated metabolites of bis(2ethylhexyl)phthalate in urine. J Chromatogr B Biomed Appl. 1996; 677(1):99-109. https://doi.org/10.1016/0378-4347(95)00439-4

57. Egestad B, Sjöberg P. Glucosidation as a new conjugation pathway for metabolites of bis(2ethylhexyl) phthalate. Drug Metab Dispos. 1992; 20(3):470-472.

58. Peck CC, Albro PW. Toxic potential of the plasticizer di(2-ethylhexyl) phthalate in the context of its disposition and metabolism in primates and man. Environ Health Perspect. 1982; 45:11-17. https://doi.org/10.1289/ehp.824511

59. Koch HM, Bolt HM, Angerer J. Di(2-ethylhexyl)phthalate (DEHP) metabolites in human urine and serum after a single oral dose of deuterium-labelled DEHP. Arch Toxicol. 2004; 78(3):123-130. https://doi.org/10.1007/s00204-003-0522-3

60. Koch HM, Bolt HM, Preuss R, Angerer J. New metabolites of di(2-ethylhexyl)phthalate (DEHP) in human urine and serum after single oral doses of deuterium-labelled DEHP. Arch Toxicol. 2005; 79(7):367-376. https://doi.org/10.1007/s00204-004-0642-4

61. Silva MJ, Samandar E, Preau JL, Jr., Needham LL, Calafat AM. Urinary oxidative metabolites of di(2-ethylhexyl) phthalate in humans. Toxicology. 2006; 219(1-3):22-32. https://doi.org/10.1016/j.tox.2005.10.018

62. Kato K, Silva MJ, Reidy JA, Hurtz D, 3rd, Malek NA, Needham LL, Nakazawa H, Barr DB, Calafat AM. Mono(2-ethyl-5-hydroxyhexyl) phthalate and mono-(2-ethyl-5-oxohexyl) phthalate 
as biomarkers for human exposure assessment to di-(2-ethylhexyl) phthalate. Environ Health Perspect. 2004; 112(3):327-330. https://doi.org/10.1289/ehp.6663

63. U.S. Consumer Product Safety Commission (CPSC). Toxicity review of DEHP. 2010. https://www.cpsc.gov/s3fs-public/ToxicityReviewOfDEHP.pdf

64. Shaffer CB, Carpenter, C.P., and H.J. Smyth. Acute and subacute toxicity of di(2-ethylhexyl) phthalate with note upon its metabolism. J Ind Hyg Toxicol. 1945; (27):130-135.

65. Shibko SI, Blumenthal H. Toxicology of phthalic acid esters used in food-packaging material. Environ Health Perspect. 1973; 3:131-137. https://doi.org/10.1289/ehp.7303131

66. Lawrence WH, Malik M, Turner JE, Singh AR, Autian J. A toxicological investigation of some acute, short-term, and chronic effects of administering di-2-ethylhexyl phthalate (DEHP) and other phthalate esters. Environ Res. 1975; 9(1):1-11. https://doi.org/10.1016/00139351(75)90043-2

67. Krauskopf LG. Studies on the toxicity of phthalates via ingestion. Environ Health Perspect. 1973; 3:61-72. https://doi.org/10.1289/ehp.730361

68. Dostal LA, Jenkins WL, Schwetz BA. Hepatic peroxisome proliferation and hypolipidemic effects of di(2-ethylhexyl)phthalate in neonatal and adult rats. Toxicol Appl Pharmacol. 1987; 87(1):81-90. https://doi.org/10.1016/0041-008x(87)90086-x

69. National Toxicology Program (NTP). NTP technical report on the carcinogenesis bioassay of di(2-ethylhexyl)phthalate (CAS No. 117-81-7) in F344 rats and B6C3F1 mice (feed study).

Research Triangle Park, NC: U.S. Department of Health and Human Services, National Institute of Environmental Health Sciences, National Toxicology Program; 1982. NTP Technical Report No. 217.

https://ntp.niehs.nih.gov/publications/reports/tr/200s/tr217/index.html?utm source=direct\&utm $\underline{\text { medium }=\text { prod\&utm campaign }=\text { ntpgolinks\&utm term }=\operatorname{tr} 217 \mathrm{abs}}$

70. Crocker JF, Safe SH, Acott P. Effects of chronic phthalate exposure on the kidney. J Toxicol Environ Health. 1988; 23(4):433-444. https://doi.org/10.1080/15287398809531126

71. Moore MR. Oncogenicity study in rats with di(2-ethylhexyl)phthalate including ancillary hepatocellular proliferation and biochemical analyses. Corning Hazleton Incorporated (CHV); 1996.

72. Moore MR. Oncogenicity study in mice with Di (2-ethylhexyl)phthalate including ancillary hepatocellular proliferation and biochemical analyses. Corning Hazleton Incorporated (CHV); 1997.

73. International Agency for Research on Cancer (IARC). Evaluation of Carcinogenic Risks to Humans: Some Industrial Chemicals. Lyon, France; 2000.

74. Ward JM, Peters JM, Perella CM, Gonzalez FJ. Receptor and nonreceptor-mediated organspecific toxicity of di(2-ethylhexyl)phthalate (DEHP) in peroxisome proliferator-activated receptor alpha-null mice. Toxicol Pathol. 1998; 26(2):240-246.

https://doi.org/10.1177/019262339802600208 
75. Maloney EK, Waxman DJ. Trans-activation of PPARalpha and PPARgamma by structurally diverse environmental chemicals. Toxicol Appl Pharmacol. 1999; 161(2):209-218.

https://doi.org/10.1006/taap.1999.8809

76. Dostal LA, Chapin RE, Stefanski SA, Harris MW, Schwetz BA. Testicular toxicity and reduced Sertoli cell numbers in neonatal rats by di(2-ethylhexyl)phthalate and the recovery of fertility as adults. Toxicol Appl Pharmacol. 1988; 95(1):104-121. https://doi.org/10.1016/s0041$\underline{008 \times(88) 80012-7}$

77. Gray TJ, Butterworth KR. Testicular atrophy produced by phthalate esters. Arch Toxicol Suppl. 1980; 4:452-455. https://doi.org/10.1007/978-3-642-67729-8_106

78. Gray TJ, Gangolli SD. Aspects of the testicular toxicity of phthalate esters. Environ Health Perspect. 1986; 65:229-235. https://doi.org/10.1289/ehp.8665229

79. Lamb JCt, Chapin RE, Teague J, Lawton AD, Reel JR. Reproductive effects of four phthalic acid esters in the mouse. Toxicol Appl Pharmacol. 1987; 88(2):255-269.

https://doi.org/10.1016/0041-008x(87)90011-1

80. Parmar D, Srivastava SP, Singh GB, Seth PK. Testicular toxicity of Di(2ethylhexyl)phthalate in developing rats. Vet Hum Toxicol. 1995; 37(4):310-313.

81. Poon R, Lecavalier P, Mueller R, Valli VE, Procter BG, Chu I. Subchronic oral toxicity of di-n-octyl phthalate and di(2-Ethylhexyl) phthalate in the rat. Food Chem Toxicol. 1997; 35(2):225-239. https://doi.org/10.1016/s0278-6915(96)00064-6

82. Sjöberg P, Bondesson U, Gray TJ, Ploen L. Effects of di-(2-ethylhexyl) phthalate and five of its metabolites on rat testis in vivo and in in vitro. Acta Pharmacol Toxicol (Copenh). 1986; 58(3):225-233. https://doi.org/10.1111/j.1600-0773.1986.tb00098.x

83. Chapin RE, Gray TJ, Phelps JL, Dutton SL. The effects of mono-(2-ethylhexyl)-phthalate on rat Sertoli cell-enriched primary cultures. Toxicol Appl Pharmacol. 1988; 92(3):467-479. https://doi.org/10.1016/0041-008x(88)90186-X

84. Svechnikov K, Svechnikova I, Söder O. Inhibitory effects of mono-ethylhexyl phthalate on steroidogenesis in immature and adult rat Leydig cells in vitro. Reprod Toxicol. 2008; 25(4):485490. https://doi.org/10.1016/j.reprotox.2008.05.057

85. Parmar D, Srivastava SP, Singh GB, Seth PK. Effect of testosterone on the testicular atrophy caused by di(2-ethylhexyl)phthalate (DEHP). Toxicol Lett. 1987; 36(3):297-308. https://doi.org/10.1016/0378-4274(87)90199-8

86. David RM, Moore MR, Finney DC, Guest D. Chronic toxicity of di(2-ethylhexyl)phthalate in rats. Toxicol Sci. 2000; 55(2):433-443. https://doi.org/10.1093/toxsci/55.2.433

87. Pugh G, Jr., Isenberg JS, Kamendulis LM, Ackley DC, Clare LJ, Brown R, Lington AW, Smith JH, Klaunig JE. Effects of di-isononyl phthalate, di-2-ethylhexyl phthalate, and clofibrate in cynomolgus monkeys. Toxicol Sci. 2000; 56(1):181-188.

https://doi.org/10.1093/toxsci/56.1.181 
88. Davis BJ, Maronpot RR, Heindel JJ. Di-(2-ethylhexyl) phthalate suppresses estradiol and ovulation in cycling rats. Toxicol Appl Pharmacol. 1994; 128(2):216-223.

https://doi.org/10.1006/taap.1994.1200

89. Ritter EJ, Scott WJ, Jr., Randall JL, Ritter JM. Teratogenicity of di(2-ethylhexyl) phthalate, 2-ethylhexanol, 2-ethylhexanoic acid, and valproic acid, and potentiation by caffeine.

Teratology. 1987; 35(1):41-46. https://doi.org/10.1002/tera.1420350107

90. Tyl RW, Price CJ, Marr MC, Kimmel CA. Developmental toxicity evaluation of dietary di(2ethylhexyl)phthalate in Fischer 344 rats and CD-1 mice. Fundam Appl Toxicol. 1988; 10(3):395412. https://doi.org/10.1016/0272-0590(88)90286-2

91. George F, Wilson, J Gonads and ducts in mammals. In: The Physiology of Reproduction. 1994. p. 3-28.

92. Byskov AG, Hoyer PE. Embryology of mammalian gonads and ducts In: Knobil E, J.D. N, editors. The Physiology of Reproduction. New York: Raven Press; 1994. p. 487-539.

93. Parks LG, Ostby JS, Lambright CR, Abbott BD, Klinefelter GR, Barlow NJ, Gray LE, Jr. The plasticizer diethylhexyl phthalate induces malformations by decreasing fetal testosterone synthesis during sexual differentiation in the male rat. Toxicol Sci. 2000; 58(2):339-349.

https://doi.org/10.1093/toxsci/58.2.339

94. Kay VR, Bloom MS, Foster WG. Reproductive and developmental effects of phthalate diesters in males. Crit Rev Toxicol. 2014; 44(6):467-498. https://doi.org/10.3109/10408444.2013.875983

95. Gray LE, Jr., Ostby J, Furr J, Price M, Veeramachaneni DN, Parks L. Perinatal exposure to the phthalates DEHP, BBP, and DINP, but not DEP, DMP, or DOTP, alters sexual differentiation of the male rat. Toxicol Sci. 2000; 58(2):350-365.

https://doi.org/10.1093/toxsci/58.2.350

96. Wolf C, Lambright C, Mann P, Price M, Cooper RL, Ostby J, Gray Jr LE. Administration of potentially antiandrogenic pesticides (procymidone, linuron, iprodione, chlozolinate, $\mathrm{p}, \mathrm{p}^{\prime}$-DDE, and ketoconazole) and toxic substances (dibutyl-and diethylhexyl phthalate, PCB 169, and ethane dimethane sulphonate) during sexual differentiation produces diverse profiles of reproductive malformations in the male rat. Toxicol Ind Health. 1999; 15(1-2):94-118. https://doi.org/10.1177/074823379901500109

97. Foster PM, Mylchreest E, Gaido KW, Sar M. Effects of phthalate esters on the developing reproductive tract of male rats. Hum Reprod Update. 2001; 7(3):231-235.

https://doi.org/10.1093/humupd/7.3.231

98. Arcadi FA, Costa C, Imperatore C, Marchese A, Rapisarda A, Salemi M, Trimarchi GR, Costa G. Oral toxicity of bis(2-ethylhexyl) phthalate during pregnancy and suckling in the LongEvans rat. Food Chem Toxicol. 1998; 36(11):963-970. https://doi.org/10.1016/s0278$\underline{6915(98) 00065-9}$ 
99. Foster PM. Disruption of reproductive development in male rat offspring following in utero exposure to phthalate esters. Int J Androl. 2006; 29(1):140-147; discussion 181-145. https://doi.org/10.1111/j.1365-2605.2005.00563.x

100. National Toxicology Program (NTP). Diethylhexylphthalate: Multigenerational reproductive assessment by continuous breeding when administered to Sprague-Dawley rats in the diet. Research Triangle Park, NC: U.S. Department of Health and Human Services, National Institute of Environmental Health Sciences, National Toxicology Program; 2004.

101. Duty SM, Calafat AM, Silva MJ, Brock JW, Ryan L, Chen Z, Overstreet J, Hauser R. The relationship between environmental exposure to phthalates and computer-aided sperm analysis motion parameters. J Androl. 2004; 25(2):293-302. https://doi.org/10.1002/j.19394640.2004.tb02790.x

102. Duty SM, Calafat AM, Silva MJ, Ryan L, Hauser R. Phthalate exposure and reproductive hormones in adult men. Hum Reprod. 2005; 20(3):604-610. https://doi.org/10.1093/humrep/deh656

103. Duty SM, Silva MJ, Barr DB, Brock JW, Ryan L, Chen Z, Herrick RF, Christiani DC, Hauser R. Phthalate exposure and human semen parameters. Epidemiology. 2003; 14(3):269277.

104. Duty SM, Singh NP, Silva MJ, Barr DB, Brock JW, Ryan L, Herrick RF, Christiani DC, Hauser R. The relationship between environmental exposures to phthalates and DNA damage in human sperm using the neutral comet assay. Environ Health Perspect. 2003; 111(9):1164-1169. https://doi.org/10.1289/ehp.5756

105. Hauser R, Williams P, Altshul L, Calafat AM. Evidence of interaction between polychlorinated biphenyls and phthalates in relation to human sperm motility. Environ Health Perspect. 2005; 113(4):425-430. https://doi.org/10.1289/ehp.7305

106. Swan SH. Environmental phthalate exposure in relation to reproductive outcomes and other health endpoints in humans. Environ Res. 2008; 108(2):177-184.

https://doi.org/10.1016/j.envres.2008.08.007

107. Swan SH, Main KM, Liu F, Stewart SL, Kruse RL, Calafat AM, Mao CS, Redmon JB, Ternand CL, Sullivan S et al. Decrease in anogenital distance among male infants with prenatal phthalate exposure. Environ Health Perspect. 2005; 113(8):1056-1061.

https://doi.org/10.1289/ehp.8100

108. Sharpe RM, Skakkebaek NE. Testicular dysgenesis syndrome: Mechanistic insights and potential new downstream effects. Fertil Steril. 2008; 89(2 Suppl):e33-38.

https://doi.org/10.1016/j.fertnstert.2007.12.026

109. Skakkebaek NE, Rajpert-De Meyts E, Main KM. Testicular dysgenesis syndrome: An increasingly common developmental disorder with environmental aspects. Hum Reprod. 2001; 16(5):972-978. https://doi.org/10.1093/humrep/16.5.972 
110. Larsen ST, Hansen JS, Thygesen P, Begtrup M, Poulsen OM, Nielsen GD. Adjuvant and immuno-suppressive effect of six monophthalates in a subcutaneous injection model with BALB/c mice. Toxicology. 2001; 169(1):37-51. https://doi.org/10.1016/s0300-483x(01)00484-x

111. Badr MZ, Shnyra A, Zoubine M, Norkin M, Herndon B, Quinn T, Miranda RN, Cunningham ML, Molteni A. Phthalate-induced liver protection against deleterious effects of the Th1 response: A potentially serious health hazard. PPAR Res. 2007; 2007:49671. https://doi.org/10.1155/2007/49671

112. Larsen ST, Nielsen GD. The adjuvant effect of di-(2-ethylhexyl) phthalate is mediated through a PPARalpha-independent mechanism. Toxicol Lett. 2007; 170(3):223-228. https://doi.org/10.1016/j.toxlet.2007.03.009

113. Tonk EC, Verhoef A, Gremmer ER, van Loveren H, Piersma AH. Relative sensitivity of developmental and immune parameters in juvenile versus adult male rats after exposure to di(2ethylhexyl) phthalate. Toxicol Appl Pharmacol. 2012; 260(1):48-57.

https://doi.org/10.1016/j.taap.2012.01.018

114. Piepenbrink MS, Hussain I, Marsh JA, Dietert RR. Developmental immunotoxicology of di-(2-ethylhexyl)phthalate (DEHP): Age-based assessment in the female rat. J Immunotoxicol. 2005; 2(1):21-31. https://doi.org/10.1080/15363750490429435

115. Butala JH, David RM, Gans G, McKee RH, Guo TL, Peachee VL, White KL, Jr. Phthalate treatment does not influence levels of IgE or Th2 cytokines in B6C3F1 mice. Toxicology. 2004; 201(1-3):77-85. https://doi.org/10.1016/j.tox.2004.04.004

116. Hansen JS, Larsen ST, Poulsen LK, Nielsen GD. Adjuvant effects of inhaled mono-2ethylhexyl phthalate in BALB/cJ mice. Toxicology. 2007; 232(1-2):79-88.

https://doi.org/10.1016/j.tox.2006.12.011

117. Larsen ST, Hansen JS, Hammer M, Alarie Y, Nielsen GD. Effects of mono-2-ethylhexyl phthalate on the respiratory tract in BALB/c mice. Hum Exp Toxicol. 2004; 23(11):537-545. https://doi.org/10.1191/0960327104ht4860a

118. Jaakkola JJ, Knight TL. The role of exposure to phthalates from polyvinyl chloride products in the development of asthma and allergies: A systematic review and meta-analysis. Environ Health Perspect. 2008; 116(7):845-853. https://doi.org/10.1289/ehp.10846

119. Nielsen J, Fahraeus C, Bensryd I, Akesson B, Welinder H, Linden K, Skerfving S. Small airways function in workers processing polyvinylchloride. Int Arch Occup Environ Health. 1989; 61(7):427-430. https://doi.org/10.1007/bf00386474

120. Jaakkola JJ, Ieromnimon A, Jaakkola MS. Interior surface materials and asthma in adults: A population-based incident case-control study. Am J Epidemiol. 2006; 164(8):742-749. https://doi.org/10.1093/aje/kwj249

121. Bornehag CG, Sundell J, Weschler CJ, Sigsgaard T, Lundgren B, Hasselgren M, HagerhedEngman L. The association between asthma and allergic symptoms in children and phthalates in house dust: A nested case-control study. Environ Health Perspect. 2004; 112(14):1393-1397. https://doi.org/10.1289/ehp.7187 
122. Kolarik B, Naydenov K, Larsson M, Bornehag CG, Sundell J. The association between phthalates in dust and allergic diseases among Bulgarian children. Environ Health Perspect. 2008; 116(1):98-103. https://doi.org/10.1289/ehp.10498

123. Kluwe WM, McConnell EE, Huff JE, Haseman JK, Douglas JF, Hartwell WV. Carcinogenicity testing of phthalate esters and related compounds by the National Toxicology Program and the National Cancer Institute. Environ Health Perspect. 1982; 45:129-133. https://doi.org/10.1289/ehp.8245129

124. David RM, Moore MR, Cifone MA, Finney DC, Guest D. Chronic peroxisome proliferation and hepatomegaly associated with the hepatocellular tumorigenesis of di(2-ethylhexyl)phthalate and the effects of recovery. Toxicol Sci. 1999; 50(2):195-205.

https://doi.org/10.1093/toxsci/50.2.195

125. David RM, Moore MR, Finney DC, Guest D. Chronic toxicity of di(2-ethylhexyl)phthalate in mice. Toxicol Sci. 2000; 58(2):377-385. https://doi.org/10.1093/toxsci/58.2.377

126. Voss C, Zerban H, Bannasch P, Berger MR. Lifelong exposure to di-(2-ethylhexyl)phthalate induces tumors in liver and testes of Sprague-Dawley rats. Toxicology. 2005; 206(3):359-371. https://doi.org/10.1016/j.tox.2004.07.016

127. Klaunig JE, Babich MA, Baetcke KP, Cook JC, Corton JC, David RM, DeLuca JG, Lai DY, McKee RH, Peters JM et al. PPARalpha agonist-induced rodent tumors: Modes of action and human relevance. Crit Rev Toxicol. 2003; 33(6):655-780.

https://doi.org/10.1080/713608372

128. National Toxicology Program (NTP). Report on Carcinogens, Fourteenth Edition. Research Triangle Park: U.S. Department of Health and Human Services, National Institute of Environmental Health Sciences, National Toxicology Program; 2016.

http://ntp.niehs.nih.gov/go/roc14

129. Grosse Y, Baan R, Secretan-Lauby B, El Ghissassi F, Bouvard V, Benbrahim-Tallaa L, Guha N, Islami F, Galichet L, Straif K et al. Carcinogenicity of chemicals in industrial and consumer products, food contaminants and flavourings, and water chlorination byproducts. Lancet Oncol. 2011; 12(4):328-329.

130. Ito Y, Yamanoshita O, Asaeda N, Tagawa Y, Lee CH, Aoyama T, Ichihara G, Furuhashi K, Kamijima M, Gonzalez FJ et al. Di(2-ethylhexyl)phthalate induces hepatic tumorigenesis through a peroxisome proliferator-activated receptor alpha-independent pathway. J Occup Health. 2007; 49(3):172-182. https://doi.org/10.1539/joh.49.172

131. Morimura K, Cheung C, Ward JM, Reddy JK, Gonzalez FJ. Differential susceptibility of mice humanized for peroxisome proliferator-activated receptor alpha to $\mathrm{Wy}-14,643$-induced liver tumorigenesis. Carcinogenesis. 2006; 27(5):1074-1080. https://doi.org/10.1093/carcin/bgi329

132. Yang Q, Ito S, Gonzalez FJ. Hepatocyte-restricted constitutive activation of PPAR alpha induces hepatoproliferation but not hepatocarcinogenesis. Carcinogenesis. 2007; 28(6):11711177. https://doi.org/10.1093/carcin/bgm046 
133. Lopez-Carrillo L, Hernandez-Ramirez RU, Calafat AM, Torres-Sanchez L, Galvan-Portillo M, Needham LL, Ruiz-Ramos R, Cebrian ME. Exposure to phthalates and breast cancer risk in northern Mexico. Environ Health Perspect. 2010; 118(4):539-544.

https://doi.org/10.1289/ehp.0901091

134. Hagmar L, Akesson B, Nielsen J, Andersson C, Lindén K, Attewell R, Möller T. Mortality and cancer morbidity in workers exposed to low levels of vinyl chloride monomer at a polyvinyl chloride processing plant. Am J Ind Med. 1990; 17(5):553-565.

https://doi.org/10.1002/ajim.4700170502

135. Hardell L, Ohlson CG, Fredrikson M. Occupational exposure to polyvinyl chloride as a risk factor for testicular cancer evaluated in a case-control study. Int J Cancer. 1997; 73(6):828-830. https://doi.org/10.1002/(sici)1097-0215(19971210)73:6<828::aid-ijc10>3.0.co;2-0

136. Ohlson CG, Hardell L. Testicular cancer and occupational exposures with a focus on xenoestrogens in polyvinyl chloride plastics. Chemosphere. 2000; 40(9-11):1277-1282. https://doi.org/10.1016/s0045-6535(99)00380-x

137. Hansen J. Risk for testicular cancer after occupational exposure to plastics. Int J Cancer. 1999; 82(6):911-912. https://doi.org/10.1002/(sici)1097-0215(19990909)82:6<911::aidijc $23>3.0 .00 ; 2-0$

138. Hardell L, Malmqvist N, Ohlson CG, Westberg H, Eriksson M. Testicular cancer and occupational exposure to polyvinyl chloride plastics: A case-control study. Int J Cancer. 2004; 109(3):425-429. https://doi.org/10.1002/ijc.11709

139. Westberg HB, Hardell LO, Malmqvist N, Ohlson CG, Axelson O. On the use of different measures of exposure-experiences from a case-control study on testicular cancer and PVC exposure. J Occup Environ Hyg. 2005; 2(7):351-356.

https://doi.org/10.1080/15459620590969046

140. Selenskas S, Teta MJ, Vitale JN. Pancreatic cancer among workers processing synthetic resins. Am J Ind Med. 1995; 28(3):385-398. https://doi.org/10.1002/ajim.4700280308

141. Huber WW, Grasl-Kraupp B, Schulte-Hermann R. Hepatocarcinogenic potential of di(2ethylhexyl)phthalate in rodents and its implications on human risk. Crit Rev Toxicol. 1996; 26(4):365-481. https://doi.org/10.3109/10408449609048302

142. International Agency for Research on Cancer (IARC). IARC monographs on the evaluation of the carcinogenic risk of chemicals to humans. Some industrial chemicals and dyestuffs. Lyon, France; 1982.

143. Caldwell JC. DEHP: Genotoxicity and potential carcinogenic mechanisms-A review. Mutat Res/Rev Mutat Res. 2012; 751(2):82-157. https://doi.org/10.1016/j.mrrev.2012.03.001

144. Tomita I, Nakamura Y, Aoki N, Inui N. Mutagenic/carcinogenic potential of DEHP and MEHP. Environ Health Perspect. 1982; 45:119-125. https://doi.org/10.1289/ehp.8245119

145. Chang YJ, Tseng CY, Lin PY, Chuang YC, Chao MW. Acute exposure to DEHP metabolite, MEHP cause genotoxicity, mutagenesis and carcinogenicity in mammalian Chinese hamster ovary cells. Carcinogenesis. 2017; 38(3):336-345. https://doi.org/10.1093/carcin/bgx009 
146. Erkekoglu P, Rachidi W, Yuzugullu OG, Giray B, Favier A, Ozturk M, Hincal F. Evaluation of cytotoxicity and oxidative DNA damaging effects of di(2-ethylhexyl)-phthalate (DEHP) and mono(2-ethylhexyl)-phthalate (MEHP) on MA-10 Leydig cells and protection by selenium. Toxicol Appl Pharmacol. 2010; 248(1):52-62.

https://doi.org/10.1016/j.taap.2010.07.016

147. Erkekoglu P, Rachidi W, De Rosa V, Giray B, Favier A, Hincal F. Protective effect of selenium supplementation on the genotoxicity of di(2-ethylhexyl)phthalate and mono(2ethylhexyl)phthalate treatment in LNCaP cells. Free Radic Biol Med. 2010; 49(4):559-566. https://doi.org/10.1016/j.freeradbiomed.2010.04.038

148. Park SY, Choi J. Cytotoxicity, genotoxicity and ecotoxicity assay using human cell and environmental species for the screening of the risk from pollutant exposure. Environ Int. 2007; 33(6):817-822. https://doi.org/10.1016/j.envint.2007.03.014

149. Zeiger E, Haworth S, Mortelmans K, Speck W. Mutagenicity testing of di(2ethylhexyl)phthalate and related chemicals in Salmonella. Environ Mutagen. 1985; 7(2):213-232. https://doi.org/10.1002/em.2860070209

150. Zeiger E, Haworth S. Tests with a preincubation modification of the Salmonella/microsome assay In: Ashby J, de Serres FJ, editors. Progress in Mutation Research: Evaluation of short-term tests for carcinogens: Report of the International Programme on Chemical Safety's Collaborative Study on In Vitro Assays Elsevier; 1985. p. 187-199.

151. Myhr B, Bowers L, Caspary WJ. Assays for the induction of gene mutations at the thymidine kinase locus in L5178Y mouse lymphoma cells in culture In: Ashby J, de Serres FJ, editors. Progress in Mutation Research: Evaluation of short-term tests for carcinogens: Report of the International Programme on Chemical Safety's Collaborative Study on In Vitro Assays Elsevier Science Publisher; 1985. p. 555-568.

152. Simon VF, Kauhanen K, Tardiff RG. Mutagenic activity of chemicals identified in drinking water In: Scott D, Bridges BA, Sobels FH, editors. Progress in Genetic Toxicology: Proceedings of the Second International Conference on Environmental Mutagens. Elsevier; 1977. p. 249-258.

153. Gulati DK, Witt K, Anderson B, Zeiger E, Shelby MD. Chromosome aberration and sister chromatid exchange tests in Chinese hamster ovary cells in vitro III: Results with 27 chemicals. Environ Mol Mutagen. 1989; 13(2):133-193. https://doi.org/10.1002/em.2850130208

154. Stenchever MA, Allen MA, Jerominski L, Petersen RV. Effects of bis(2-ethylhexyl) phthalate on chromosomes of human leukocytes and human fetal lung cells. J Pharm Sci. 1976; 65(11):1648-1651. https://doi.org/10.1002/jps.2600651121

155. Abe S, Sasaki M. Chromosome aberrations and sister chromatid exchanges in Chinese hamster cells exposed to various chemicals. J Natl Cancer Inst. 1977; 58(6):1635-1641. https://doi.org/10.1093/jnci/58.6.1635

156. Ishidate M, Jr., Odashima S. Chromosome tests with 134 compounds on Chinese hamster cells in vitro--a screening for chemical carcinogens. Mutat Res. 1977; 48(3-4):337-353.

https://doi.org/10.1016/0027-5107(77)90177-4 
157. Phillips BJ, James TEB, Gangoli SD. Genotoxicity studies of di(2-ethylhexyl)phthalate and its metabolites in CHO cells. Mutat Res. 1982; 102(3):297-304. https://doi.org/10.1016/01651218(82)90139-2

158. Yoon JS, Mason JM, Valencia R, Woodruff RC, Zimmering S. Chemical mutagenesis testing in Drosophila. IV. Results of 45 coded compounds tested for the National Toxicology Program. Environ Mutagen. 1985; 7(3):349-367. https://doi.org/10.1002/em.2860070310

159. Zimmering S, Mason JM, Valencia R. Chemical mutagenesis testing in Drosophila. VII. Results of 22 coded compounds tested in larval feeding experiments. Environ Mol Mutagen. 1989; 14(4):245-251. https://doi.org/10.1002/em.2850140406

160. Kitamoto S, Matsuyama R, Uematsu Y, Ogata K, Ota M, Yamada T, Miyata K, Kimura J, Funabashi H, Saito K. Genotoxicity evaluation of benzene, di(2-ethylhexyl) phthalate, and trisodium ethylenediamine tetraacetic acid monohydrate using a combined rat comet/micronucleus assays. Mutat Res Genet Toxicol Environ Mutagen. 2015; 786-788:137143. https://doi.org/10.1016/j.mrgentox.2015.05.002

161. Akagi J-I, Toyoda T, Cho Y-M, Mizuta Y, Nohmi T, Nishikawa A, Ogawa K. Validation study of the combined repeated-dose toxicity and genotoxicity assay using gpt delta rats. Cancer Sci. 2015; 106(5):529-541. https://doi.org/10.1111/cas.12634

162. Kanki K, Nishikawa A, Masumura K, Umemura T, Imazawa T, Kitamura Y, Nohmi T, Hirose M. In vivo mutational analysis of liver DNA in gpt delta transgenic rats treated with the hepatocarcinogens N-nitrosopyrrolidine, 2-amino-3-methylimidazo[4,5-f]quinoline, and di(2ethylhexyl)phthalate. Mol Carcinog. 2005; 42(1):9-17. https://doi.org/10.1002/mc.20061

163. Smith-Oliver T, Butterworth BE. Correlation of the carcinogenic potential of di(2ethylhexyl)phthalate (DEHP) with induced hyperplasia rather than with genotoxic activity. Mutat Res. 1987; 188(1):21-28. https://doi.org/10.1016/0165-1218(87)90110-8

164. Singh AR, Lawrence WH, Autian J. Mutagenic and antifertility sensitivities of mice to di-2ethylhexyl phthalate (DEHP) and dimethoxyethyl phthalate (DMEP). Toxicology and Applied Pharmacology. 1974; 29(1):35-46. https://doi.org/10.1016/0041-008X(74)90159-8

165. Jäckh R, Rhodes C, Grasso P, Carter JT. Genotoxicity studies on di-(2-ethylhexyl) phthalate and adipate and toxicity studies on di-(2-ethylhexyl) phthalate in the rat and marmoset. Food Chem Toxicol. 1984; 22(2):151-155. https://doi.org/10.1016/0278-6915(84)90096-6

166. Maronpot RR, Boorman GA. Interpretation of rodent hepatocellular proliferative alterations and hepatocellular tumors in chemical safety assessment. Toxicol Pathol. 1982; 10(2):71-78. https://doi.org/10.1177/019262338201000210

167. Boorman GA, Haseman JK, Waters MD, Hardisty JF, Sills RC. Quality review procedures necessary for rodent pathology databases and toxicogenomic studies: The National Toxicology Program experience. Toxicol Pathol. 2002; 30(1):88-92. https://doi.org/10.1080/01926230252824752 
168. McConnell E, Solleveld HA, Swenberg JA, Boorman GA. Guidelines for combining neoplasms for evaluation of rodent carcinogenesis studies. J Natl Cancer Inst. 1986; 76(2):283289.

169. U.S. Environmental Protection Agency (USEPA). Benchmark Dose Software (BMDS). Version 3.1.2.; 2020. https://www.epa.gov/bmds

170. U.S. Environmental Protection Agency (USEPA). Benchmark dose software (BMDS) version 3.1.1. user guide EPA/600/R-18/310. July 2019. 2019.

171. Kaplan EL, Meier P. Nonparametric estimation from incomplete observations. J Am Stat Assoc. 1958; 53(282):457-481. https://doi.org/10.2307/2281868

172. Tarone RE. Tests for trend in life table analysis. Biometrika. 1975; 62(3):679-690.

173. Cox DR. Regression models and life-tables. J R Stat Soc Series B. 1972; 34(2):187-202.

174. Bailer AJ, Portier CJ. Effects of treatment-induced mortality and tumor-induced mortality on tests for carcinogenicity in small samples. Biometrics. 1988; 44(2):417-431.

175. Piegorsch W, Bailer A. Statistics for Environmental Biology and Toxicology: Section 6.3.2. London, UK: Chapman and Hall; 1997.

176. Portier CJ, Bailer AJ. Testing for increased carcinogenicity using a survival-adjusted quantal response test. Fundam Appl Toxicol. 1989; 12(4):731-737.

177. Portier CJ, Hedges JC, Hoel DG. Age-specific models of mortality and tumor onset for historical control animals in the National Toxicology Program's carcinogenicity experiments. Cancer Res. 1986; 46(9):4372-4378.

178. Bieler GS, Williams RL. Ratio estimates, the delta method, and quantal response tests for increased carcinogenicity. Biometrics. 1993; 49(3):793-801.

179. Nam JM. A simple approximation for calculating sample sizes for detecting linear trend in proportions. Biometrics. 1987; 43(3):701-705.

180. Rao JN, Scott AJ. A simple method for the analysis of clustered binary data. Biometrics. 1992; 48(2):577-585.

181. Fung KY, Krewski D, Rao JN, Scott AJ. Tests for trend in developmental toxicity experiments with correlated binary data. Risk Anal. 1994; 14(4):639-648.

182. Gart JJ, Chu KC, Tarone RE. Statistical issues in interpretation of chronic bioassay tests for carcinogenicity. J Natl Cancer Inst. 1979; 62(4):957-974.

183. Dixon W, Massey F. Introduction to Statistical Analysis. New York, NY: McGraw Hill Book Company Inc; 1957. http://dx.doi.org/10.2307/2332898

184. Tukey J. Easy summaries - numerical and graphical. Exploratory Data Analysis. Reading, MA: Addison-Wesley; 1977. p. 43-44. 
185. Dunnett CW. A multiple comparison procedure for comparing several treatments with a control. J Am Stat Assoc. 1955; 50(272):1096-1121.

http://dx.doi.org/10.1080/01621459.1955.10501294

186. Williams D. A test for differences between treatment means when several dose levels are compared with a zero dose control. Biometrics. 1971; 27(1):103-117.

http://dx.doi.org/10.2307/2528930

187. Williams D. The comparison of several dose levels with a zero dose control. Biometrics. 1972; 28(2):519-531. http://dx.doi.org/10.2307/2556164

188. Shirley E. A non-parametric equivalent of Williams' test for contrasting increasing dose levels of a treatment. Biometrics. 1977; 33(2):386-389. http://dx.doi.org/10.2307/2529789

189. Williams DA. A note on Shirley's nonparametric test for comparing several dose levels with a zero-dose control. Biometrics. 1986; 42(1):183-186. http://dx.doi.org/10.2307/2531254

190. Dunn OJ. Multiple comparisons using rank sums. Technometrics. 1964; 6(3):241-252. http://dx.doi.org/10.1080/00401706.1964.10490181

191. Jonckheere A. A distribution-free k-sample test against ordered alternatives. Biometrika. 1954; 41:133-145. http://dx.doi.org/10.1093/biomet/41.1-2.133

192. Hsu JC. The factor analytic approach to simultaneous inference in the general linear model. J Comput Graph Stat. 1992; 1(2):151-168. https://doi.org/10.1080/10618600.1992.10477011

193. Haseman JK. Data analysis: Statistical analysis and use of historical control data. Regul Toxicol Pharmacol. 1995; 21(1):52-59; discussion 81-56. https://doi.org/10.1006/rtph.1995.1009

194. Haseman JK, Rao GN. Effects of corn oil, time-related changes, and inter-laboratory variability on tumor occurrence in control Fischer 344 (F344/N) rats. Toxicol Pathol. 1992; 20(1):52-60. https://doi.org/10.1177/019262339202000107

195. Haseman JK. Value of historical controls in the interpretation of rodent tumor data. Drug Inf J. 1992; 26(2):191-200. https://doi.org/10.1177/009286159202600210

196. Code of Federal Regulations (CFR). 21(Part 58).

197. Miller JA, Miller EC. Ultimate chemical carcinogens as reactive mutagenic electrophiles In: Hiatt HH, Watson JD, Winsten JA, editors. Origins of Human Cancer. Cold Spring Harbor, NY: Cold Spring Harbor Laboratory; 1977. p. 605-627.

198. Straus DS. Somatic mutation, cellular differentiation, and cancer causation. J Natl Cancer Inst. 1981; 67:233-241.

199. Crawford BD. Perspectives on the somatic mutation model of carcinogenesis In: Mehlman MA, Flamm WG, Lorentzen RJ, editors. Advances in Modern Environmental Toxicology Mechanisms and Toxicity of Chemical Carcinogens and Mutagens. Princeton, NJ: Princeton Scientific Publishing Co. Inc; 1985. p. 13-59.

200. Ashby J, Tennant RW. Definitive relationships among chemical structure, carcinogenicity and mutagenicity for 301 chemicals tested by the US NTP. Mutat Res. 1991; 257(3):229-306. 
201. Witt KL, Knapton A, Wehr CM, Hook GJ, Mirsalis J, Shelby MD, MacGregor JT. Micronucleated erythrocyte frequency in peripheral blood of $\mathrm{B} 6 \mathrm{C} 3 \mathrm{~F}(1)$ mice from short-term, prechronic, and chronic studies of the NTP carcinogenesis bioassay program. Environ Mol Mutagen. 2000; 36(3):163-194.

202. National Toxicology Program (NTP). TR-601: Technical report pathology tables and curves pathology tables, survival and growth curves from NTP long term, genetic toxicology, and bench mark dose analyses of neoplastic lesions studies. Research Triangle Park, NC: U.S. Department of Health and Human Services, National Institute of Environmental Health Sciences, National Toxicology Program; 2020. https://doi.org/10.22427/NTP-DATA-TR-601

203. Araki A, Mitsui T, Miyashita C, Nakajima T, Naito H, Ito S, Sasaki S, Cho K, Ikeno T, Nonomura K et al. Association between maternal exposure to di(2-ethylhexyl) phthalate and reproductive hormone levels in fetal blood: The Hokkaido study on environment and children's health. PLoS One. 2014; 9(10):e109039-e109039. https://doi.org/10.1371/journal.pone.0109039

204. Latini G, De Felice C, Presta G, Del Vecchio A, Paris I, Ruggieri F, Mazzeo P. Exposure to di(2-ethylhexyl)phthalate in humans during pregnancy. Neonatology. 2003; 83(1):22-24. https:// doi.org/10.1159/000067012

205. Li X, Sun H, Yao Y, Zhao Z, Qin X, Duan Y, Wang L. Distribution of phthalate metabolites between paired maternal-fetal samples. Environ Sci Technol. 2018; 52(11):6626-6635. https://doi.org/10.1021/acs.est.8b00838

206. Mose T, Knudsen LE, Hedegaard M, Mortensen GK. Transplacental transfer of monomethyl phthalate and mono(2-ethylhexyl) phthalate in a human placenta perfusion system. Int $\mathbf{J}$ Toxicol. 2007; 26(3):221-229. https://doi.org/10.1080/10915810701352721

207. Dalgaard M, Ostergaard G, Lam HR, Hansen EV, Ladefoged O. Toxicity study of di(2ethylhexyl)phthalate (DEHP) in combination with acetone in rats. Pharmacol Toxicol. 2000; 86(2):92-100. https://doi.org/10.1034/j.1600-0773.2000.pto860208.x

208. Dalsenter PR, Santana GM, Grande SW, Andrade AJ, Araujo SL. Phthalate affect the reproductive function and sexual behavior of male Wistar rats. Hum Exp Toxicol. 2006; 25(6):297-303. https://doi.org/10.1191/0960327105ht624oa

209. Pocar P, Fiandanese N, Secchi C, Berrini A, Fischer B, Schmidt JS, Schaedlich K, Borromeo V. Exposure to di(2-ethyl-hexyl) phthalate (DEHP) in utero and during lactation causes long-term pituitary-gonadal axis disruption in male and female mouse offspring. Endocrinology. 2012; 153(2):937-948. https://doi.org/10.1210/en.2011-1450

210. National Toxicology Program (NTP). Diethylhexylphthalate: Multigenerational reproductive assessment by continuous breeding when administered to Sprague-Dawley rats in the diet. Research Triangle Park, NC: U.S. Department of Health and Human Services, National Institute of Environmental Health Sciences, National Toxicology Program; 2005. PB2005107575.

211. National Toxicology Program (NTP). NTP technical report on the toxicology and carcinogenesis studies of perfluorooctanoic acid (CASRN 335-67-1) administered in feed to Sprague Dawley (Hsd:Sprague Dawley® $\mathrm{SD}{ }^{\circledR}$ ) rats. Research Triangle Park, NC: U.S. 
Department of Health and Human Services, National Institute of Environmental Health Sciences, National Toxicology Program; 2020. NTP Technical Report No. 598.

https://ntp.niehs.nih.gov/publications/reports/tr/500s/tr598/index.html?utm source=direct\&utm $\underline{\text { medium }=\text { prod \&utm campaign }=\text { ntpgolinks\&utm term }=\operatorname{tr} 598 \mathrm{abs}}$

212. Rusyn I, Corton JC. Mechanistic considerations for human relevance of cancer hazard of di(2-ethylhexyl) phthalate. Mutat Res. 2012; 750(2):141-158.

https://doi.org/10.1016/j.mrrev.2011.12.004

213. National Toxicology Program (NTP). NTP toxicology and carcinogenesis studies of butyl benzyl phthalate (CAS No. 85-68-7) in F334/N rats (Feed studies). Research Triangle Park, NC: U.S. Department of Health and Human Services, National Institute of Environmental Health Sciences, National Toxicology Program; 1997. NTP Technical Report No. 458. https://ntp.niehs.nih.gov/publications/reports/tr/400s/tr458/index.html?utm source=direct\&utm $\underline{\text { medium }=\text { prod\&utm campaign }=\text { ntpgolinks \&utm term }=\text { tr } 458 \mathrm{abs}}$

214. Biegel LB, Hurtt ME, Frame SR, O'Connor JC, Cook JC. Mechanisms of extrahepatic tumor induction by peroxisome proliferators in male CD rats. Toxicol Sci. 2001; 60(1):44-55. https://doi.org/10.1093/toxsci/60.1.44

215. Bell RH, Jr., Kuhlmann ET, Jensen RT, Longnecker DS. Overexpression of cholecystokinin receptors in azaserine-induced neoplasms of the rat pancreas. Cancer Res. 1992; 52(12):32953299.

216. Longnecker DS. Interface between adaptive and neoplastic growth in the pancreas. Gut. 1987; 28 Suppl:253-258. https://doi.org/10.1136/gut.28.suppl.253

217. Povoski SP, Zhou W, Longnecker DS, Roebuck BD, Bell RH, Jr. Stimulation of growth of azaserine-induced putative preneoplastic lesions in rat pancreas is mediated specifically by way of cholecystokinin-A receptors. Cancer Res. 1993; 53(17):3925-3929.

218. Gray LE, Foster P. Significance of experimental studies for assessing adverse effects of endocrine-disrupting chemicals. Pure Appl Chem. 2003; 75(11-12):2125-2141.

https://doi.org/10.1351/pac200375112125

219. Gray Jr LE, Furr J, Tatum-Gibbs KR, Lambright C, Sampson H, Hannas BR, Wilson VS, Hotchkiss A, Foster PM. Establishing the "biological relevance" of dipentyl phthalate reductions in fetal rat testosterone production and plasma and testis testosterone levels. Toxicol Sci. 2016; 149(1):178-191. https://doi.org/10.1093/toxsci/kfv224

220. Howdeshell KL, Furr J, Lambright CR, Rider CV, Wilson VS, Gray LE, Jr. Cumulative effects of dibutyl phthalate and diethylhexyl phthalate on male rat reproductive tract development: Altered fetal steroid hormones and genes. Toxicol Sci. 2007; 99(1):190-202. https://doi.org/10.1093/toxsci/kfm069

221. Borch J, Axelstad M, Vinggaard AM, Dalgaard M. Diisobutyl phthalate has comparable anti-androgenic effects to di-n-butyl phthalate in fetal rat testis. Toxicol Lett. 2006; 163(3):183190. https://doi.org/10.1016/j.toxlet.2005.10.020 
222. Welsh M, Saunders PTK, Fisken M, Scott HM, Hutchison GR, Smith LB, Sharpe RM. Identification in rats of a programming window for reproductive tract masculinization, disruption of which leads to hypospadias and cryptorchidism. J Clin Invest. 2008; 118(4):1479-1490. https://doi.org/10.1172/JCI34241

223. Nef S, Parada LF. Cryptorchidism in mice mutant for Insl3. Nat Genet. 1999; 22(3):295299.

224. Zimmermann S, Steding G, Emmen JMA, Brinkmann AO, Nayernia K, Holstein AF, Engel $\mathrm{W}$, Adham IM. Targeted disruption of the Insl3 gene causes bilateral cryptorchidism. Mol Endocrinol. 1999; 13(5):681-691. https://doi.org/10.1210/mend.13.5.0272

225. Wilson VS, Lambright C, Furr J, Ostby J, Wood C, Held G, Gray LE. Phthalate esterinduced gubernacular lesions are associated with reduced insl3 gene expression in the fetal rat testis. Toxicol Lett. 2004; 146(3):207-215. https://doi.org/10.1016/j.toxlet.2003.09.012

226. Barlow NJ, McIntyre BS, Foster PMD. Male reproductive tract lesions at 6, 12, and 18 months of age following in utero exposure to di(n-butyl) phthalate. Toxicol Pathol. 2004; 32(1):79-90. https://doi.org/10.1080/01926230490265894

227. Furr JR, Lambright CS, Wilson VS, Foster PM, Gray LE, Jr. A short-term in vivo screen using fetal testosterone production, a key event in the phthalate adverse outcome pathway, to predict disruption of sexual differentiation. Toxicol Sci. 2014; 140(2):403-424. https://doi.org/10.1093/toxsci/kfu081

228. Fisher JS, Macpherson S, Marchetti N, Sharpe RM. Human 'testicular dysgenesis syndrome': A possible model using in-utero exposure of the rat to dibutyl phthalate. Hum Reprod. 2003; 18(7):1383-1394. https://doi.org/10.1093/humrep/deg273

229. van den Driesche S, Kilcoyne KR, Wagner I, Rebourcet D, Boyle A, Mitchell R, McKinnell $\mathrm{C}$, Macpherson S, Donat R, Shukla CJ et al. Experimentally induced testicular dysgenesis syndrome originates in the masculinization programming window. JCI Insight. 2017; 2(6):e91204. https://doi.org/10.1172/jci.insight.91204

230. Sohval AR. Testicular dysgenesis as an etiologic factor in cryptorchidism. J Urol. 1954; 72(4):693-702. https://doi.org/10.1016/s0022-5347(17)67649-3

231. Sohval AR. Testicular dysgenesis in relation to neoplasm of the testicle. J Urol. 1956; 75(2):285-291. https://doi.org/10.1016/s0022-5347(17)66809-5

232. Hoei-Hansen CE, Holm M, Rajpert-De Meyts E, Skakkebaek NE. Histological evidence of testicular dysgenesis in contralateral biopsies from 218 patients with testicular germ cell cancer. J Pathol. 2003; 200(3):370-374. https://doi.org/10.1002/path.1372

233. Gray TJB, Butterworth KR, Gaunt IF, Grasso P, Gangolli SD. Short-term toxicity study of di-(2-ethylhexyl) phthalate in rats. Food Cosmet Toxicol. 1977; 15(5):389-399.

https://doi.org/10.1016/S0015-6264(77)80003-5

234. Clegg ED, Cook JC, Chapin RE, Foster PM, Daston GP. Leydig cell hyperplasia and adenoma formation: Mechanisms and relevance to humans. Reprod Toxicol. 1997; 11(1):107121. https://doi.org/10.1016/s0890-6238(96)00203-1 
235. Cook JC, Klinefelter GR, Hardisty JF, Sharpe RM, Foster PM. Rodent Leydig cell tumorigenesis: A review of the physiology, pathology, mechanisms, and relevance to humans. Crit Rev Toxicol. 1999; 29(2):169-261. https://doi.org/10.1080/10408449991349203

236. Creasy D, Bube A, de Rijk E, Kandori H, Kuwahara M, Masson R, Nolte T, Reams R, Regan K, Rehm S et al. Proliferative and nonproliferative lesions of the rat and mouse male reproductive system. Toxicol Pathol. 2012; 40(6 Suppl):40s-121s.

https://doi.org/10.1177/0192623312454337

237. Wei Z, Song L, Wei J, Chen T, Chen J, Lin Y, Xia W, Xu B, Li X, Chen X et al. Maternal exposure to di-(2-ethylhexyl)phthalate alters kidney development through the renin-angiotensin system in offspring. Toxicol Lett. 2012; 212(2):212-221.

https://doi.org/10.1016/j.toxlet.2012.05.023

238. Khorana AA, Fine RL. Pancreatic cancer and thromboembolic disease. Lancet Oncol. 2004; 5(11):655-663. https://doi.org/10.1016/S1470-2045(04)01606-7

239. Kamijo Y, Hora K, Nakajima T, Kono K, Takahashi K, Ito Y, Higuchi M, Kiyosawa K, Shigematsu H, Gonzalez FJ et al. Peroxisome proliferator-activated receptor alpha protects against glomerulonephritis induced by long-term exposure to the plasticizer di-(2ethylhexyl)phthalate. J Am Soc Nephrol. 2007; 18(1):176-188.

https://doi.org/10.1681/asn.2006060597

240. Organisation for Economic Co-operation and Development (OECD). OECD guidelines for testing of chemicals. Test guideline 479. Test no. 479: Genetic toxicology: In vitro sister chromatid exchange assay in mammalian cells. 2014. https://www.oecdilibrary.org/environment/test-no-479-genetic-toxicology-in-vitro-sister-chromatid-exchangeassay-in-mammalian-cells 9789264071384-en

241. McFee AF, Lowe KW, San Sebastian JR. Improved sister-chromatid differentiation using paraffin-coated bromodeoxyuridine tablets in mice. Mutat Res. 1983; 119(1):83-88. https://doi.org/10.1016/0165-7992(83)90042-8

242. McFee AF, Tice RR, Shelby MD. In vivo cytogenetic activity of diphenylhydantoin in mice. Mutat Res. 1992; 278(1):61-68. https://doi.org/10.1016/0165-1218(92)90286-9

243. Shelby MD, Gulati DK, Tice RR, Wojciechowski JP. Results of tests for micronuclei and chromosomal aberrations in mouse bone marrow cells with the human carcinogens 4aminobiphenyl, treosulphan, and melphalan. Environ Mol Mutagen. 1989; 13(4):339-342. https://doi.org/10.1002/em.2850130410

244. Margolin BH, Resnick MA, Rimpo JY, Archer P, Galloway SM, Bloom AD, Zeiger E. Statistical analyses for in vitro cytogenetic assays using Chinese hamster ovary cells. Environ Mutagen. 1986; 8(2):183-204. https://doi.org/10.1002/em.2860080203

245. MacGregor JT, Wehr CM, Henika PR, Shelby MD. The in vivo erythrocyte micronucleus test: Measurement at steady state increases assay efficiency and permits integration with toxicity studies. Fundam Appl Toxicol. 1990; 14(3):513-522. https://doi.org/10.1016/0272-

0590(90)90255-i 


\section{Appendix A. Chemical Characterization and Dose Formulation Studies}

\section{Table of Contents}

A.1. Procurement and Characterization of Di(2-ethylhexyl) Phthalate

A-2

A.2. Preparation and Analysis of Dose Formulations....

\section{Tables}

Table A-1. Chromatography Systems Used in the Two-year Feed Studies of

Di(2-ethyhexyl) Phthalate

Table A-2. Preparation and Storage of Dose Formulations in the Two-year Feed Studies of Di(2-ethylhexyl) Phthalate

Table A-3. Results of Analyses of Dose Formulations Administered to Rats in the Perinatal and Postweaning Two-year Feed Study of Di(2-ethylhexyl)

Phthalate

Table A-4. Results of Analyses of Dose Formulations Administered to Rats in the Postweaning-only Two-year Feed Study of Di(2-ethylhexyl) Phthalate

\section{Figures}

Figure A-1. Reference Infrared Absorption Spectrum of Di(2-ethylhexyl) Phthalate

Figure A-2. Fourier Transformed ${ }^{1} \mathrm{H}$ Nuclear Magnetic Resonance Spectrum of Sample of Di(2-ethylhexyl) Phthalate (Lot 01514TH)

Figure A-3. Fourier Transformed ${ }^{13} \mathrm{C}$ Nuclear Magnetic Resonance Spectrum of Sample of Di(2-ethylhexyl) Phthalate (Lot 01514TH) 


\section{A.1. Procurement and Characterization of Di(2-ethylhexyl) Phthalate}

Di(2-ethylhexyl) phthalate (DEHP) was obtained from Aldrich Chemical Company Inc. (St. Louis, MO) in a single lot (lot 01514TH) received in two shipments. The first shipment $(10 \mathrm{~L})$ was received on December 12, 2008 and used for chemical characterization. The second shipment (250 L) was received on November 4, 2009 and used for the dose formulations in the 2-year studies and chemical reanalysis. Identity, purity, and stability analyses were conducted by the analytical chemistry laboratory at RTI International (Research Triangle Park, NC). Reports on analyses performed in support of the DEHP studies are on file at the National Institute of Environmental Health Sciences.

The appearance (clear liquid) and density of lot $01514 \mathrm{TH}\left(0.976 \mathrm{~g} / \mathrm{mL}\right.$ at $\left.21.9^{\circ} \mathrm{C}\right)$ matched that of DEHP $\left(0.985 \mathrm{~g} / \mathrm{mL}\right.$ at $\left.25^{\circ} \mathrm{C}\right)$. Galbraith Laboratories (Knoxville, TN) performed the boiling point and elemental analyses of lot $01514 \mathrm{TH}$. While the elemental analysis confirmed the anticipated relative ratios, the experimental boiling point $\left(330.9^{\circ} \mathrm{C}\right)$ was considerably lower than that reported in the literature $\left(384^{\circ} \mathrm{C}\right)$. Using a different method, the results $\left(335^{\circ} \mathrm{C}, 760 \mathrm{~mm} \mathrm{Hg}\right)$ from RTI International were consistent with Galbraith Laboratories. A precise molecular mass was measured using research-grade high-resolution mass spectrometry (HRMS) at the University of South Carolina Mass Spectrometry Facility (Columbia, SC). The observed mass values (390.2772) were within acceptable limits $(\leq 5 \mathrm{ppm})$ of the calculated mass (390.2770).

The identity of lot $01514 \mathrm{TH}$ was confirmed using infrared (IR) spectroscopy, ${ }^{1} \mathrm{H}$ and ${ }^{13} \mathrm{C}$ nuclear magnetic resonance (NMR) spectroscopy, and gas chromatography (GC) with MS detection. The IR spectrum was in good agreement with the structure of DEHP and with the reference spectrum from the National Institute of Advanced Industrial Science and Technology Spectral Database for Organic Compounds (SDBS No. 2266) for DEHP (Figure A-1). ${ }^{1} \mathrm{H}$ and ${ }^{13} \mathrm{C}$ NMR spectra (Figure A-2, Figure A-3) were consistent with the structure of DEHP and the prediction from the Advanced Chemistry Development Spectral Prediction Program (Version 10.02, Toronto, Ontario, Canada) for DEHP. GC/MS identified the major peak from the $10 \mathrm{~L}$ shipment of lot $01514 \mathrm{TH}$ as DEHP using fragmentation pattern analysis and comparison with the National Institute of Standards and Technology (NIST) reference spectrum (No. 311338) for DEHP (Table A-1, System A). The GC/MS spectra correlated well with the structure of DEHP.

The moisture content of lot $01514 \mathrm{TH}$ was determined by Karl Fisher titration. The purity was determined using ultra-performance liquid chromatography (UPLC) with photodiode array detector (PDA) and using GC with flame ionization detection (FID) (Table A-1, Systems B and $\mathrm{C}$, respectively). The Karl Fisher titration yielded a water content of $0.145 \%$. UPLC/PDA analysis demonstrated one major peak accounting for $99.7 \%$ and one minor peak accounting for $0.2 \%$ of the total integrated area. GC/FID analysis also found one major peak accounting for $99.7 \%$ and one minor peak accounting for $0.3 \%$ of the total integrated area. An additional $\mathrm{GC} / \mathrm{MS}$ analysis of the test chemical was performed in an attempt to identify the minor component in the chromatographic profile (Table A-1, System A). The fragmentation of the minor impurity peak agreed with the NIST reference spectrum (No. 312137) for mono(2-ethylhexyl) phthalate.

Accelerated stability studies were conducted by the analytical chemistry laboratory using samples of lot $01514 \mathrm{TH}$ stored at ambient temperature (approximately $22^{\circ} \mathrm{C}$ ), refrigerated temperature (approximately $5^{\circ} \mathrm{C}$ ), and elevated temperature (approximately $60^{\circ} \mathrm{C}$ ) in amber vials 
sealed with foil-lined caps. After 14 days, samples were analyzed by GC/FID (Table A-1, System C). Stability of DEHP was confirmed for at least 2 weeks when stored in sealed glass vials at temperatures from $5^{\circ} \mathrm{C}$ to $60^{\circ} \mathrm{C}$.

Upon receipt of the $250 \mathrm{~L}$ shipment used for the 2-year studies, the bulk chemical of lot $01514 \mathrm{TH}$ was homogenized by shaking each of the $550 \mathrm{~L}$ plastic jugs for approximately 2 minutes and transferred to $704 \mathrm{~L}$ amber glass storage bottles, which were stored at room temperature.

Prior to using the bulk chemical from the $250 \mathrm{~L}$ shipment of lot $01514 \mathrm{TH}$ for dose formulations, the identity was confirmed using the same GC/MS system with comparison to the reference spectrum and an aliquot of the test article from the 10L shipment (Table A-1, System A). The GC/MS analysis of the $250 \mathrm{~L}$ shipment of lot 01514TH demonstrated one major peak accounting for $99.9 \%$ of the total integrated area. Periodic reanalysis of the bulk chemical lot $01514 \mathrm{TH}$ was performed prior to and during the animal studies by the laboratory using high-performance liquid chromatography (HPLC) with ultraviolet (UV) detection (Table A-1, System D), and no degradation of the test chemical was detected.

\section{A.2. Preparation and Analysis of Dose Formulations}

The base diet was meal feed purchased from Zeigler Brothers, Inc. (Gardners, PA). The perinatal and postweaning study (Study 1) utilized NIH-07 feed (2 lots milled March and April 2011) in addition to NTP-2000 feed (25 lots milled April 2011-March 2013). The postweaning-only study (Study 2) utilized NTP-2000 feed (25 lots milled December 2010-December 2012). In addition to determining the suitability of the vehicles for feeding the animals, analysis of the NTP-2000 feed extract performed by the study laboratory using liquid chromatography mass spectrometry (LC/MS) confirmed that the vehicle did not contain the test article DEHP.

Dose formulations were prepared monthly by mixing DEHP with feed (Table A-2). For the perinatal and postweaning study, formulations were prepared at concentrations of $0,300,1,000$, 3,000 , and 10,000 ppm in both NIH-07 feed (May 4, May 24, and June 15, 2011) and in NTP-2000 feed (31 formulations; June 2011-June 2013). For the postweaning-only study, formulations were prepared in NTP-2000 feed at concentrations of $0,300,1,000,3,000$, and 10,000 ppm (31 formulations; February 2011-February 2013). The plastic bags used by the study laboratory in the preparation and storage of blank and dosed feed were determined to have no levels of DEHP above the limit of detection of the assay (1.27 ppm).

Homogeneity studies were performed on the 25 and $10,000 \mathrm{ppm}$ dose formulations in both $25 \mathrm{-kg}$ NIH-07 feed batch sizes and 25-kg NTP-2000 feed batch sizes by the analytical chemistry laboratory using UPLC/PDA (Table A-1, System B). Additional homogeneity studies of the 300 and 10,000 ppm dose formulations in a 72-kg NIH-07 feed batch size and 300, 3,000, and 10,000 ppm dose formulations in a 92-kg NTP-2000 feed batch sizes were performed before the animal studies by the study laboratory using HPLC/UV (Table A-1, System D). All formulations analyzed were determined to be homogenous and of appropriate concentration.

Stability studies conducted by the chemistry laboratory of the $25 \mathrm{ppm} \mathrm{NIH-07}$ and $25 \mathrm{ppm}$ NTP-2000 dose formulations confirmed the stability of DEHP after 42 days at room, refrigerated, or frozen temperatures. Stability was also confirmed under simulated dosing 
conditions (room temperature, exposure to air and light for 7 days, in absence of excreta, and in presence of excreta). Control and dosed formulations were stored in individual plastic bag-lined containers at room temperature (approximately $25^{\circ} \mathrm{C}$ ) and were used within 42 days of preparation.

Periodic analyses of the preadministration dose formulations of DEHP were conducted by the study laboratory every 1 to 3 months to determine purity, while postadministration (animal room) samples were analyzed about every 1 to 7 months (Table A-3, Table A-4). All preadministration formulations were within $10 \%$ of the target concentrations. For the perinatal and postweaning study, all postadministration dose formulations of DEHP were within 10\% of target concentrations. For the postweaning-only study (Study 2), all postadministration dose formulations of DEHP were within 10\% of target concentrations except for the 1,000 ppm dose formulation prepared on July 30, 2012, collected from residual feed in the feeder that was $12.3 \%$ below the target concentration.

Table A-1. Chromatography Systems Used in the Two-year Feed Studies of Di(2-ethyhexyl) Phthalate

\begin{tabular}{llll}
\hline Chromatography & Detection System & Column & Mobile Phase \\
\hline
\end{tabular}

System A

Gas chromatography

Mass selective detector

J\&W DB-1 $(25 \mathrm{~m} \times 0.32 \mathrm{~mm}$ ID, $0.25 \mu \mathrm{m}$ film thickness)

Helium, $1.65 \mathrm{~mL} / \mathrm{min}$ flow rate

System B

Ultra-performance liquid chromatography

Photodiode array detector (205 to $400 \mathrm{~nm}$, extracted at $225 \mathrm{~nm}$ )

A: Methanol

B: Water Gradient program:
Waters Acquity UPLC BEH Phenyl

$(50 \mathrm{~mm} \times 2.1 \mathrm{~mm}$ ID, $1.7 \mu \mathrm{m}$ particle size), with Waters Acquity In-Line Filter $(0.2 \mu \mathrm{m})$ A:B $25: 75$ to $75: 25$ in 3 min, hold at 78:22 for $1 \mathrm{~min}$, ramp to 100:0 in $1 \mathrm{~min}$, hold at 100:0 for $1 \mathrm{~min}$, reverse to $25: 75$ in $0.5 \mathrm{~min}$, hold at 25:75 for $1.5 \mathrm{~min}$ $0.6 \mathrm{~mL} / \mathrm{min}$ flow rate

\section{System C}

Gas chromatography

Flame ionization detection $\left(325^{\circ} \mathrm{C}\right)$
J\&W HP-5 (30 m × $0.32 \mathrm{~mm}$ ID, $0.25 \mu \mathrm{m}$ film thickness)
Helium, $1 \mathrm{~mL} / \mathrm{min}$ flow rate 


\begin{tabular}{|c|c|c|c|}
\hline Chromatography & Detection System & Column & Mobile Phase \\
\hline \multicolumn{4}{|l|}{ System D } \\
\hline $\begin{array}{l}\text { High-performance } \\
\text { liquid chromatography }\end{array}$ & Ultraviolet $(225 \mathrm{~nm})$ & $\begin{array}{l}\text { Thermo Scientific Hypersil Phenyl } \\
(250 \mathrm{~mm} \times 4.6 \mathrm{~mm} \text { ID, } 5 \mu \mathrm{m} \\
\text { particle size) with Hypersil Phenyl } \\
\text { guard ( } 5 \mu \mathrm{m} \text { particle size) }\end{array}$ & $\begin{array}{l}\text { A: Methanol } \\
\text { B: ASTM Type I } \\
\text { Water } \\
\text { Gradient program: } \\
\text { A:B } 70: 30 \text { to } 85: 15 \text { in } \\
5 \mathrm{~min}, \text { ramp to } 100: 0 \\
\text { in } 4 \mathrm{~min} \text {, hold at } 100: 0 \\
\text { for } 4 \mathrm{~min} \text {, reverse to } \\
70: 30 \text { in } 0.1 \mathrm{~min} \text {, hold } \\
\text { at } 70: 30 \text { for } 10.9 \text { min } \\
1.0 \mathrm{~mL} / \mathrm{min} \text { flow rate }\end{array}$ \\
\hline
\end{tabular}

$\overline{\mathrm{ID}}=$ internal diameter; UPLC $=$ ultra-performance liquid chromatography; ASTM = American Society for Testing and Materials.

Table A-2. Preparation and Storage of Dose Formulations in the Two-year Feed Studies of Di(2-ethylhexyl) Phthalate

\section{Preparation}

Stock solutions of di(2-ethylhexyl) phthalate (DEHP) were created by weighing an appropriate amount of lot $01514 \mathrm{TH}$ and adding it to a volumetric flask. Acetone was used to bring the solution to volume. Flasks of stocks solution were sealed and shaken until the chemical was dissolved (at least 10 inversions). An initial formulation premix was created by weighing an appropriate amount of feed (NIH-07 or NTP-2000) into a mixing bowl. A portion of the stock DEHP solution was slowly poured onto the feed and then stirred for 2 minutes at a low setting using a Hobart mixer. The mixer was stopped, and the remaining stock solution was poured onto the feed. The stock container was rinsed with acetone twice and the rinses were poured onto the feed. The premix feed was stirred under a nitrogen stream with a flow rate of 10 liters per minute for approximately an hour to encourage cyclonic flow and to ensure complete evaporation of the acetone. The formulation blends were prepared by adding half of the required blank feed to a twin shell blender and then evenly covering with the premix. The sides were "rinsed" twice with the remaining blank feed and added to the blender. The final formulation was mixed in the blender for 15 minutes. The dose formulations were prepared approximately every 4 weeks.

\section{Chemical Lot Number}

$01514 \mathrm{TH}$

\section{Maximum Storage Time}

42 days

\section{Storage Conditions}

Stored in sealed plastic bag-lined container at $\sim 25^{\circ} \mathrm{C}$

\section{Study Laboratory}

Battelle (Columbus, $\mathrm{OH}$ )

Table A-3. Results of Analyses of Dose Formulations Administered to Rats in the Perinatal and Postweaning Two-year Feed Study of Di(2-ethylhexyl) Phthalate

\begin{tabular}{lcccc}
\hline Date Prepared & Date Analyzed & $\begin{array}{c}\text { Target } \\
\text { Concentration (ppm) }\end{array}$ & $\begin{array}{c}\text { Determined } \\
\text { Concentration (ppm) }\end{array}$ & $\begin{array}{c}\text { Difference from } \\
\text { Target (\%) }\end{array}$ \\
\hline May 4, 2011 & May 5, 2011 & 0 & BLOQ & NA \\
& & 300 & $309 \pm 5$ & 3.0 \\
& 1,000 & $1,020 \pm 20$ & 2.0 \\
\hline
\end{tabular}


Di(2-ethylhexyl) Phthalate, NTP TR 601

\begin{tabular}{|c|c|c|c|c|}
\hline Date Prepared & Date Analyzed & $\begin{array}{c}\text { Target } \\
\text { Concentration (ppm) }\end{array}$ & $\begin{array}{c}\text { Determined } \\
\text { Concentration }(\mathbf{p p m})^{\mathrm{a}}\end{array}$ & $\begin{array}{l}\text { Difference from } \\
\text { Target }(\%)\end{array}$ \\
\hline \multirow{5}{*}{ June 6, 2011} & \multirow{5}{*}{ June 6, 2011} & 3,000 & $3,170 \pm 70$ & 5.7 \\
\hline & & 10,000 & $10,200 \pm 100$ & 2.0 \\
\hline & & 0 & BLOQ & NA \\
\hline & & 300 & 291.5 & -2.8 \\
\hline & & 1,000 & 959.5 & -4.1 \\
\hline \multirow{5}{*}{ August 17, 2011} & \multirow{5}{*}{ August 19, 2011} & 3,000 & 2,885 & -3.8 \\
\hline & & 10,000 & 9,790 & -2.1 \\
\hline & & 0 & BLOQ & NA \\
\hline & & 300 & 292.5 & -2.5 \\
\hline & & 1,000 & 982.5 & -1.8 \\
\hline \multirow{5}{*}{ October 31, 2011} & \multirow{5}{*}{ November 3, 2011} & 3,000 & 2,930 & -2.3 \\
\hline & & 10,000 & 9,805 & -2.0 \\
\hline & & 0 & BLOQ & NA \\
\hline & & 300 & 287.5 & -4.2 \\
\hline & & 1,000 & 922 & -7.8 \\
\hline \multirow{5}{*}{ January 12, 2012} & \multirow{5}{*}{ January 13, 2012} & 3,000 & 2,875 & -4.2 \\
\hline & & 10,000 & 9,560 & -4.4 \\
\hline & & 0 & BLOQ & NA \\
\hline & & 300 & 305 & 1.7 \\
\hline & & 1,000 & 993 & -0.7 \\
\hline \multirow{5}{*}{ March 26, 2012} & \multirow{5}{*}{ March 29, 2012} & 3,000 & 2,990 & -0.3 \\
\hline & & 10,000 & 10,100 & 1.0 \\
\hline & & 0 & BLOQ & NA \\
\hline & & 300 & 307.5 & 2.5 \\
\hline & & 1,000 & 1,020 & 2.0 \\
\hline \multirow{5}{*}{ June 6, 2012} & \multirow{5}{*}{ June 7, 2012} & 3,000 & 3,045 & 1.5 \\
\hline & & 10,000 & 10,350 & 3.5 \\
\hline & & 0 & BLOQ & NA \\
\hline & & 300 & 295 & -1.7 \\
\hline & & 1,000 & 983 & -1.7 \\
\hline \multirow{5}{*}{ July 30, 2012} & \multirow{5}{*}{ August 1, 2012} & 3,000 & 2,990 & -0.3 \\
\hline & & 10,000 & 9,850 & -1.5 \\
\hline & & 0 & BLOQ & NA \\
\hline & & 300 & 300.5 & 0.2 \\
\hline & & 1,000 & 997.5 & -0.3 \\
\hline
\end{tabular}


Di(2-ethylhexyl) Phthalate, NTP TR 601

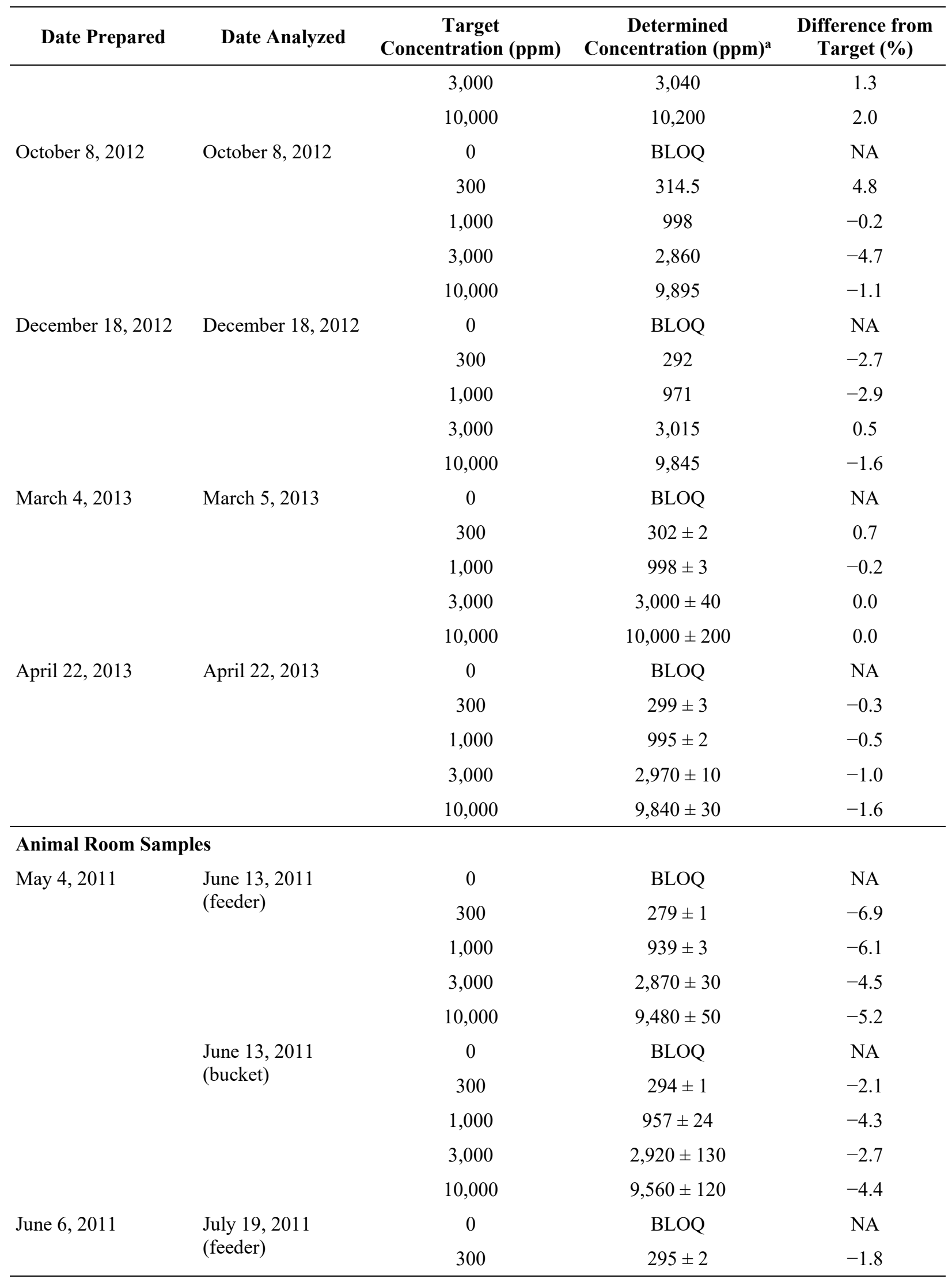


Di(2-ethylhexyl) Phthalate, NTP TR 601

\begin{tabular}{|c|c|c|c|c|}
\hline Date Prepared & Date Analyzed & $\begin{array}{c}\text { Target } \\
\text { Concentration (ppm) }\end{array}$ & $\begin{array}{c}\text { Determined } \\
\text { Concentration }(\mathbf{p p m})^{\mathrm{a}}\end{array}$ & $\begin{array}{l}\text { Difference from } \\
\text { Target }(\%)\end{array}$ \\
\hline \multirow{16}{*}{ January 12, 2012} & \multirow{7}{*}{$\begin{array}{l}\text { July 19, } 2011 \\
\text { (bucket) }\end{array}$} & 1,000 & $960 \pm 1$ & -4.0 \\
\hline & & 3,000 & $2,880 \pm 20$ & -4.0 \\
\hline & & 10,000 & $10,000 \pm 0$ & 0.0 \\
\hline & & 0 & BLOQ & NA \\
\hline & & 300 & $302 \pm 1$ & 0.5 \\
\hline & & 1,000 & $1,000 \pm 10$ & 0.3 \\
\hline & & 3,000 & $3,000 \pm 30$ & 0.1 \\
\hline & \multirow{6}{*}{$\begin{array}{l}\text { February 23, } 2012 \\
\text { (feeder) }\end{array}$} & 10,000 & $9,770 \pm 120$ & -0.3 \\
\hline & & 0 & BLOQ & NA \\
\hline & & 300 & $298 \pm 1$ & -0.6 \\
\hline & & 1,000 & $958 \pm 5$ & -4.2 \\
\hline & & 3,000 & $2,750 \pm 30$ & -8.3 \\
\hline & & 10,000 & $9,270 \pm 120$ & -7.3 \\
\hline & \multirow{3}{*}{$\begin{array}{l}\text { February 23, } 2012 \\
\text { (bucket) }\end{array}$} & 0 & BLOQ & NA \\
\hline & & 300 & $297 \pm 3$ & -0.9 \\
\hline & & 1,000 & $980 \pm 6$ & -2.0 \\
\hline \multirow{6}{*}{ July 30, 2012} & \multirow{6}{*}{$\begin{array}{l}\text { September 5, } 2012 \\
\text { (feeder) }\end{array}$} & 3,000 & $2,900 \pm 30$ & -3.4 \\
\hline & & 10,000 & $9,360 \pm 130$ & -6.4 \\
\hline & & 0 & BLOQ & NA \\
\hline & & 300 & $288 \pm 1$ & -4.1 \\
\hline & & 1,000 & $1,020 \pm 60$ & 2.2 \\
\hline & & 3,000 & $2,840 \pm 60$ & -5.3 \\
\hline \multirow{13}{*}{ March 4, 2013} & \multirow{3}{*}{$\begin{array}{l}\text { September 5, } 2012 \\
\text { (bucket) }\end{array}$} & 10,000 & $9,190 \pm 110$ & -8.1 \\
\hline & & 0 & BLOQ & NA \\
\hline & & 300 & $288 \pm 2$ & -4.1 \\
\hline & \multirow{7}{*}{$\begin{array}{l}\text { April 15, } 2013 \\
\text { (feeder) }\end{array}$} & 1,000 & $1,000 \pm 80$ & 0.0 \\
\hline & & 3,000 & $2,830 \pm 130$ & -5.7 \\
\hline & & 10,000 & $9,920 \pm 280$ & -0.8 \\
\hline & & 0 & BLOQ & NA \\
\hline & & 300 & $281 \pm 2$ & -6.2 \\
\hline & & 1,000 & $909 \pm 4$ & -9.1 \\
\hline & & 3,000 & $2,740 \pm 20$ & -8.6 \\
\hline & \multirow{3}{*}{$\begin{array}{l}\text { April 15, } 2013 \\
\text { (bucket) }\end{array}$} & 10,000 & $9,510 \pm 20$ & -4.9 \\
\hline & & 0 & BLOQ & NA \\
\hline & & 300 & $289 \pm 0$ & -3.7 \\
\hline
\end{tabular}




\begin{tabular}{|c|c|c|c|c|}
\hline Date Prepared & Date Analyzed & $\begin{array}{c}\text { Target } \\
\text { Concentration (ppm) }\end{array}$ & $\begin{array}{c}\text { Determined } \\
\text { Concentration }(\mathbf{p p m})^{\mathrm{a}}\end{array}$ & $\begin{array}{l}\text { Difference from } \\
\text { Target }(\%)\end{array}$ \\
\hline & & 1,000 & NS & NA \\
\hline & & 3,000 & $2,910 \pm 70$ & -3.0 \\
\hline & & 10,000 & $10,200 \pm 200$ & 2.0 \\
\hline
\end{tabular}

$\mathrm{BLOQ}=$ below the limit of quantification; $\mathrm{NA}=$ not applicable; $\mathrm{NS}=$ no sample collected.

aPreadministration samples are an average of triplicate analysis on two sample collections from the same preparation date.

Animal room samples are an average and standard deviation of triplicate analysis of a single sample.

Table A-4. Results of Analyses of Dose Formulations Administered to Rats in the Postweaning-only Two-year Feed Study of Di(2-ethylhexyl) Phthalate

\begin{tabular}{|c|c|c|c|c|}
\hline Date Prepared & Date Analyzed & $\begin{array}{c}\text { Target } \\
\text { Concentration (ppm) }\end{array}$ & $\begin{array}{c}\text { Determined } \\
\text { Concentration }(\mathbf{p p m})^{\mathrm{a}}\end{array}$ & $\begin{array}{l}\text { Difference from } \\
\text { Target }(\%)\end{array}$ \\
\hline \multirow[t]{5}{*}{ February 2, 2011} & February 5, 2011 & 0 & BLOQ & NA \\
\hline & & 300 & $295 \pm 5$ & -1.7 \\
\hline & & 1,000 & $987 \pm 38$ & -1.3 \\
\hline & & 3,000 & $3,040 \pm 10$ & 1.3 \\
\hline & & 10,000 & $10,300 \pm 300$ & 3.0 \\
\hline \multirow[t]{5}{*}{ March 28, 2011} & March 28, 2011 & 0 & BLOQ & NA \\
\hline & & 300 & $300 \pm 3$ & 0.0 \\
\hline & & 1,000 & $991 \pm 3$ & -0.9 \\
\hline & & 3,000 & $2,990 \pm 10$ & -0.3 \\
\hline & & 10,000 & $9,880 \pm 190$ & -1.2 \\
\hline \multirow[t]{5}{*}{ June 6, 2011} & June 6, 2011 & 0 & BLOQ & NA \\
\hline & & 300 & 291.5 & -2.8 \\
\hline & & 1,000 & 959.5 & -4.1 \\
\hline & & 3,000 & 2,885 & -3.8 \\
\hline & & 10,000 & 9,790 & -2.1 \\
\hline \multirow[t]{5}{*}{ August 17, 2011} & August 19, 2011 & 0 & BLOQ & NA \\
\hline & & 300 & 292.5 & -2.5 \\
\hline & & 1,000 & 982.5 & -1.8 \\
\hline & & 3,000 & 2,930 & -2.3 \\
\hline & & 10,000 & 9,805 & -2.0 \\
\hline \multirow[t]{5}{*}{ October 31, 2011} & November 3, 2011 & 0 & BLOQ & NA \\
\hline & & 300 & 287.5 & -4.2 \\
\hline & & 1,000 & 922 & -7.8 \\
\hline & & 3,000 & 2,875 & -4.2 \\
\hline & & 10,000 & 9,560 & -4.4 \\
\hline \multirow[t]{4}{*}{ January 12, 2012} & January 13, 2012 & 0 & BLOQ & NA \\
\hline & & 300 & 305 & 1.7 \\
\hline & & 1,000 & 993 & -0.7 \\
\hline & & 3,000 & 2,990 & -0.3 \\
\hline
\end{tabular}


Di(2-ethylhexyl) Phthalate, NTP TR 601

\begin{tabular}{|c|c|c|c|c|}
\hline Date Prepared & Date Analyzed & $\begin{array}{c}\text { Target } \\
\text { Concentration (ppm) }\end{array}$ & $\begin{array}{c}\text { Determined } \\
\text { Concentration (ppm) }\end{array}$ & $\begin{array}{l}\text { Difference from } \\
\text { Target }(\%)\end{array}$ \\
\hline \multirow{6}{*}{ March 26, 2012} & \multirow{6}{*}{ March 29, 2012} & 10,000 & 10,100 & 1.0 \\
\hline & & 0 & BLOQ & NA \\
\hline & & 300 & 307.5 & 2.5 \\
\hline & & 1,000 & 1,020 & 2.0 \\
\hline & & 3,000 & 3,045 & 1.5 \\
\hline & & 10,000 & 10,350 & 3.5 \\
\hline \multirow[t]{5}{*}{ June 6, 2012} & \multirow[t]{5}{*}{ June 7, 2012} & 0 & BLOQ & NA \\
\hline & & 300 & 295 & -1.7 \\
\hline & & 1,000 & 983 & -1.7 \\
\hline & & 3,000 & 2,990 & -0.3 \\
\hline & & 10,000 & 9,850 & -1.5 \\
\hline \multirow[t]{5}{*}{ July 30,2012} & \multirow[t]{5}{*}{ August 1, 2012} & 0 & BLOQ & NA \\
\hline & & 300 & 300.5 & 0.2 \\
\hline & & 1,000 & 997.5 & -0.3 \\
\hline & & 3,000 & 3,040 & 1.3 \\
\hline & & 10,000 & 10,200 & 2.0 \\
\hline \multirow[t]{5}{*}{ October 8, 2012} & \multirow[t]{5}{*}{ October 8, 2012} & 0 & BLOQ & NA \\
\hline & & 300 & 314.5 & 4.8 \\
\hline & & 1,000 & 998 & -0.2 \\
\hline & & 3,000 & 2,860 & -4.7 \\
\hline & & 10,000 & 9,895 & -1.1 \\
\hline \multirow[t]{5}{*}{ December 18, 2012} & \multirow[t]{5}{*}{ December 18, 2012} & 0 & BLOQ & NA \\
\hline & & 300 & 292 & -2.7 \\
\hline & & 1,000 & 971 & -2.9 \\
\hline & & 3,000 & 3,015 & 0.5 \\
\hline & & 10,000 & 9,845 & -1.6 \\
\hline \multicolumn{5}{|c|}{ Animal Room Samples } \\
\hline \multirow[t]{10}{*}{ February 2, 2011} & \multirow{5}{*}{$\begin{array}{l}\text { March 17, } 2011 \\
\text { (feeder) }\end{array}$} & 0 & BLOQ & NA \\
\hline & & 300 & $293 \pm 6$ & -2.2 \\
\hline & & 1,000 & $933 \pm 4$ & -6.7 \\
\hline & & 3,000 & $2,830 \pm 30$ & -5.6 \\
\hline & & 10,000 & $9,430 \pm 280$ & -5.7 \\
\hline & \multirow{5}{*}{$\begin{array}{l}\text { March 17, } 2011 \\
\text { (bucket) }\end{array}$} & 0 & BLOQ & NA \\
\hline & & 300 & $294 \pm 6$ & -1.9 \\
\hline & & 1,000 & $972 \pm 11$ & -2.8 \\
\hline & & 3,000 & $2,890 \pm 40$ & -3.6 \\
\hline & & 10,000 & $9,460 \pm 140$ & -5.4 \\
\hline \multirow[t]{2}{*}{ June 6, 2011} & \multirow{2}{*}{$\begin{array}{l}\text { July 19, } 2011 \\
\text { (feeder) }\end{array}$} & 0 & BLOQ & NA \\
\hline & & 300 & $283 \pm 3$ & -5.7 \\
\hline
\end{tabular}




\begin{tabular}{|c|c|c|c|c|}
\hline Date Prepared & Date Analyzed & $\begin{array}{c}\text { Target } \\
\text { Concentration (ppm) }\end{array}$ & $\begin{array}{c}\text { Determined } \\
\text { Concentration }(\mathbf{p p m})^{\mathrm{a}}\end{array}$ & $\begin{array}{l}\text { Difference from } \\
\text { Target }(\%)\end{array}$ \\
\hline & \multirow{8}{*}{$\begin{array}{l}\text { July 19, } 2011 \\
\text { (bucket) }\end{array}$} & 1,000 & $973 \pm 3$ & -2.7 \\
\hline & & 3,000 & $2,950 \pm 10$ & -1.7 \\
\hline & & 10,000 & $9,780 \pm 10$ & -2.2 \\
\hline & & 0 & BLOQ & NA \\
\hline & & 300 & $302 \pm 1$ & 0.5 \\
\hline & & 1,000 & $1,000 \pm 10$ & 0.3 \\
\hline & & 3,000 & $3,000 \pm 30$ & 0.1 \\
\hline & & 10,000 & $9,770 \pm 120$ & -0.3 \\
\hline \multirow[t]{10}{*}{ January 12, 2012} & \multirow{5}{*}{$\begin{array}{l}\text { February 23, } 2012 \\
\text { (feeder) }\end{array}$} & 0 & BLOQ & NA \\
\hline & & 300 & $299 \pm 4$ & -0.2 \\
\hline & & 1,000 & $925 \pm 1$ & -7.5 \\
\hline & & 3,000 & $2,790 \pm 30$ & -6.9 \\
\hline & & 10,000 & $9,260 \pm 110$ & -7.4 \\
\hline & \multirow{5}{*}{$\begin{array}{l}\text { February 23, } 2012 \\
\text { (bucket) }\end{array}$} & 0 & BLOQ & NA \\
\hline & & 300 & $297 \pm 3$ & -0.9 \\
\hline & & 1,000 & $980 \pm 6$ & -2.0 \\
\hline & & 3,000 & $2,900 \pm 30$ & -3.4 \\
\hline & & 10,000 & $9,360 \pm 130$ & -6.4 \\
\hline \multirow[t]{10}{*}{ July 30, 2012} & \multirow{5}{*}{$\begin{array}{l}\text { September 5, } 2012 \\
\text { (feeder) }\end{array}$} & 0 & BLOQ & NA \\
\hline & & 300 & $282 \pm 4$ & -6.1 \\
\hline & & 1,000 & $877 \pm 35$ & -12.3 \\
\hline & & 3,000 & $2,850 \pm 10$ & -5.1 \\
\hline & & 10,000 & $9,200 \pm 250$ & -8.0 \\
\hline & \multirow{5}{*}{$\begin{array}{l}\text { September 5, } 2012 \\
\text { (bucket) }\end{array}$} & 0 & BLOQ & NA \\
\hline & & 300 & $288 \pm 2$ & -4.1 \\
\hline & & 1,000 & $1,000 \pm 80$ & 0.0 \\
\hline & & 3,000 & $2,830 \pm 130$ & -5.7 \\
\hline & & 10,000 & $9,920 \pm 280$ & -0.8 \\
\hline
\end{tabular}

$\mathrm{BLOQ}=$ below the limit of quantification; $\mathrm{NA}=$ not applicable.

aPreadministration samples are an average of triplicate analysis on two sample collections from the same preparation date. Animal room samples are an average and standard deviation of triplicate analysis of a single sample. 


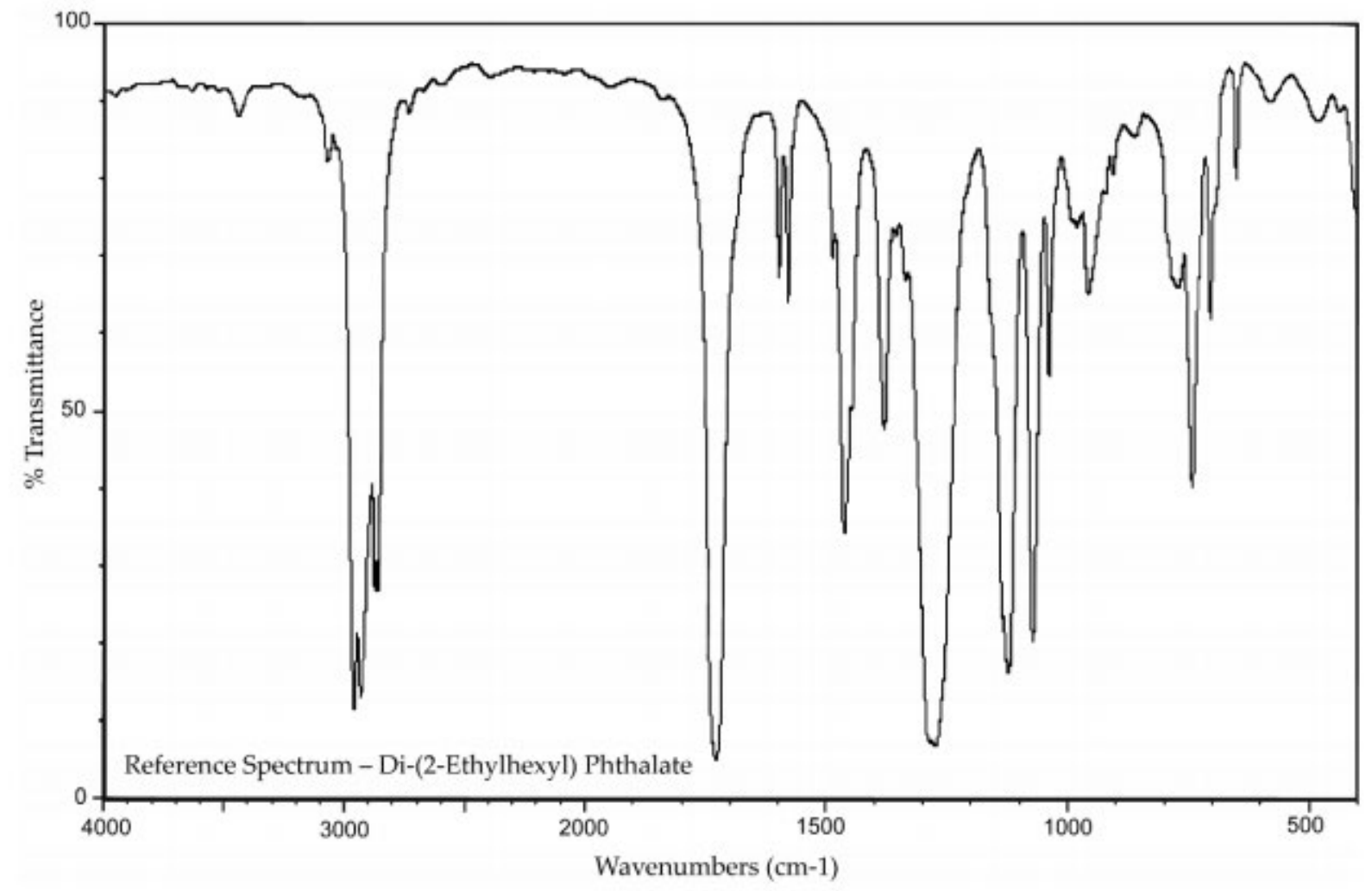

Figure A-1. Reference Infrared Absorption Spectrum of Di(2-ethylhexyl) Phthalate

Reference spectra: National Institute of Advanced Industrial Science and Technology Spectral Database for Organic Compounds. SDBS No. 2266, https://sdbs.db.aist.go.jp/sdbs/cgi-bin/landingpage?spcode=IR-NIDA-08692 (accessed Mar 19, 2009). 


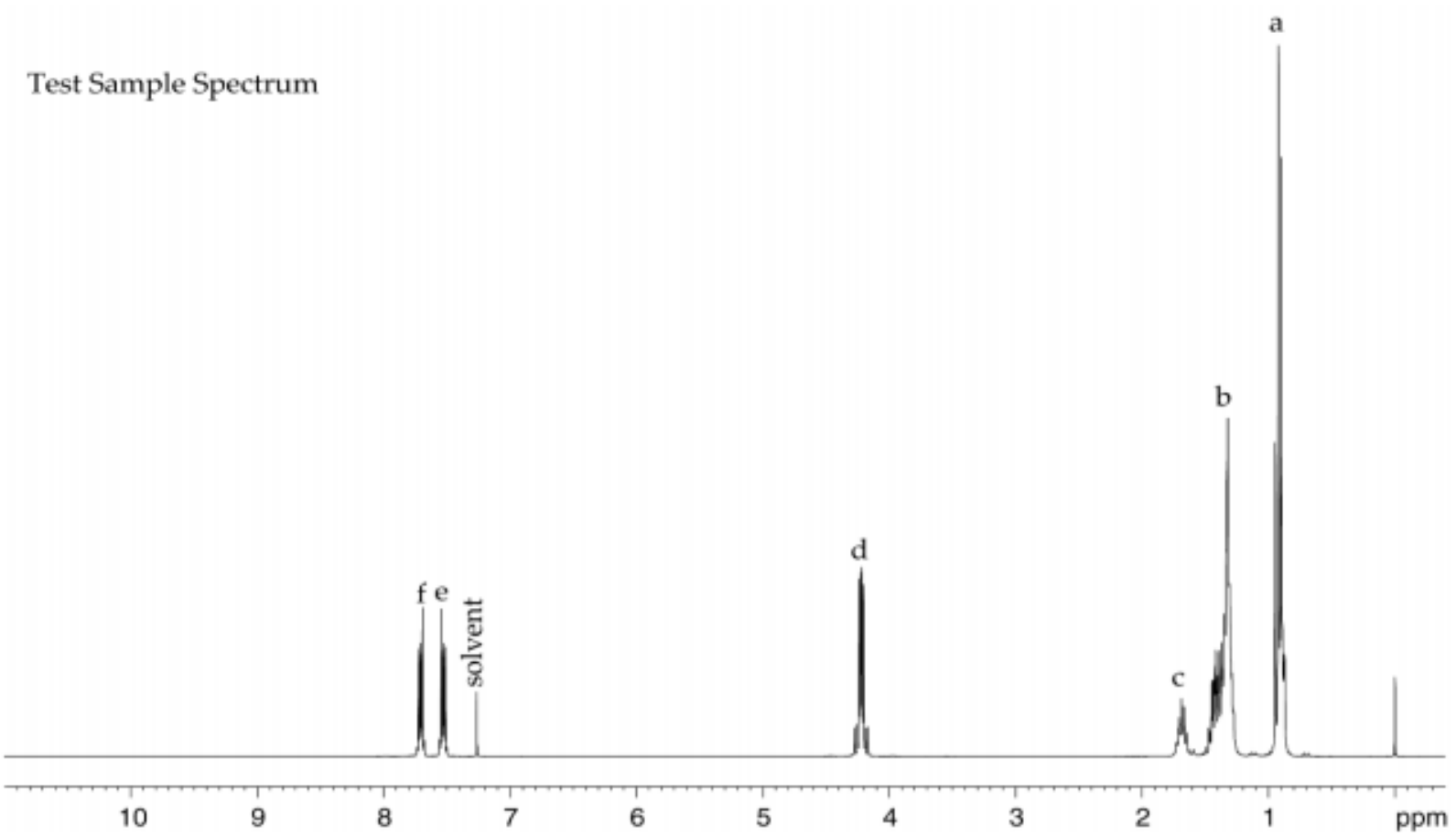

Figure A-2. Fourier Transformed ${ }^{1} \mathrm{H}$ Nuclear Magnetic Resonance Spectrum of Sample of Di(2-ethylhexyl) Phthalate (Lot 01514TH)

Reference spectra: National Institute of Advanced Industrial Science and Technology Spectral Database for Organic Compounds. SDBS No. 2266, https://sdbs.db.aist.go.jp/sdbs/cgi-bin/landingpage?spcode=NMR-HSP-03-645 (accessed Sep 22, 2009). 


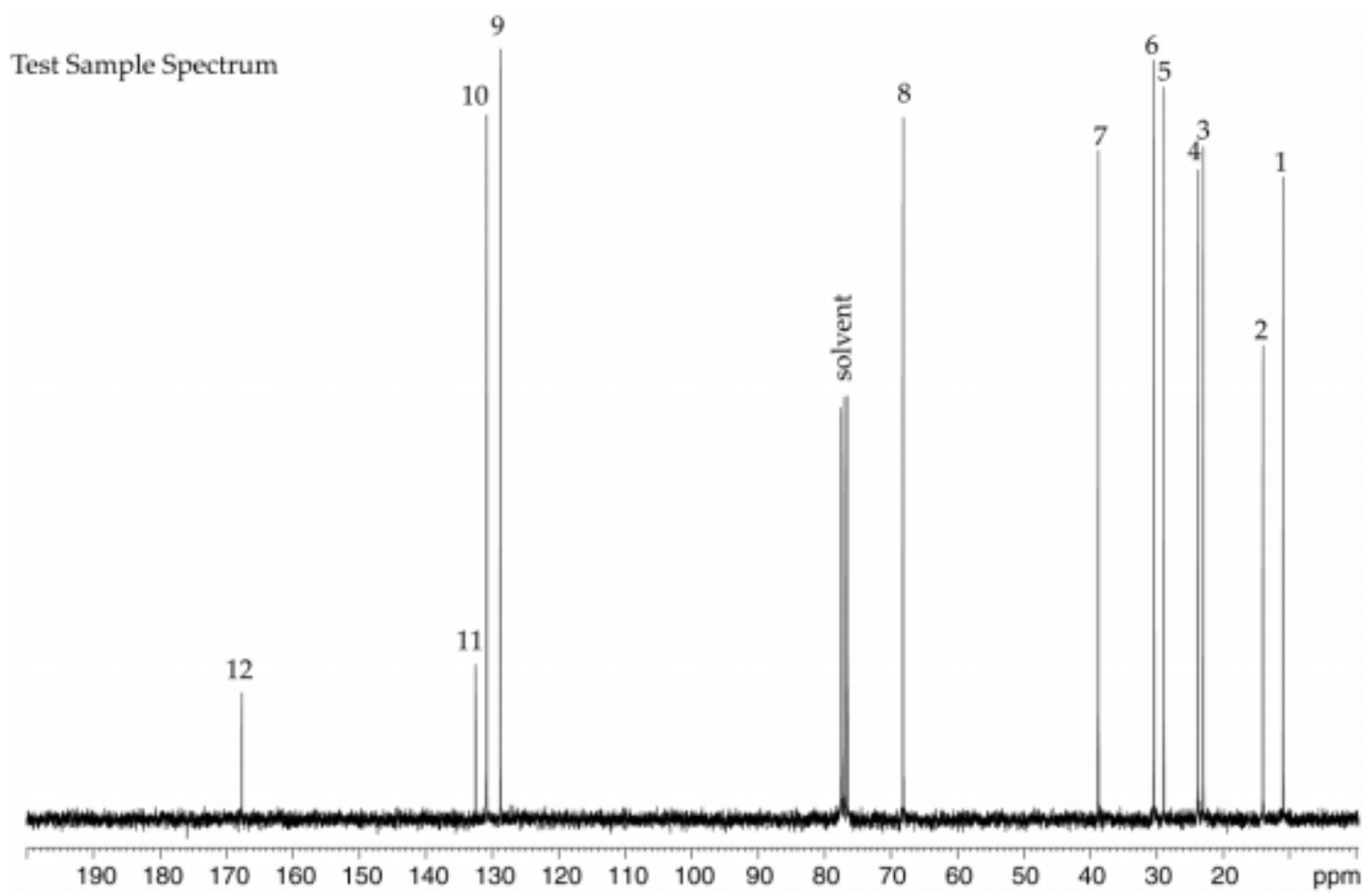

Figure A-3. Fourier Transformed ${ }^{13} \mathrm{C}$ Nuclear Magnetic Resonance Spectrum of Sample of Di(2-ethylhexyl) Phthalate (Lot 01514TH)

Reference spectra: National Institute of Advanced Industrial Science and Technology Spectral Database for Organic Compounds. SDBS No. 2266, https://sdbs.db.aist.go.jp/sdbs/cgi-bin/landingpage?spcode=NMR-CDS-05-148 (accessed Sep 22, 2009). 


\section{Appendix B. Ingredients, Nutrient Composition, and Contaminant Levels in NIH-07 and NTP-2000 Rat Rations \\ Table of Contents}

B.1. NIH-07 Feed.

B-2

B.2. NTP-2000 Feed

\section{Tables}

Table B-1. Ingredients of NIH-07 Rat Ration

B-2

Table B-2. Vitamins and Minerals in NIH-07 Rat Ration

Table B-3. Nutrient Composition of NIH-07 Rat Ration

Table B-4. Contaminant Levels in NIH-07 Rat Ration ...

Table B-5. Ingredients of NTP-2000 Rat Ration

Table B-6. Vitamins and Minerals in NTP-2000 Rat Ration

Table B-7. Nutrient Composition of NTP-2000 Rat Ration

Table B-8. Contaminant Levels in NTP-2000 Rat Ration 


\section{B.1. NIH-07 Feed}

Table B-1. Ingredients of NIH-07 Rat Ration

\begin{tabular}{lc}
\hline \multicolumn{1}{c}{ Ingredients } & Percent by Weight \\
\hline Ground Hard Winter Wheat & 23.00 \\
Ground \#2 Yellow Shelled Corn & 24.25 \\
Wheat Middlings & 10.0 \\
Oat Hulls & 0.0 \\
Alfalfa Meal (Dehydrated, 17\% Protein) & 4.0 \\
Purified Cellulose & 0.0 \\
Soybean Meal (49\% Protein) & 12.0 \\
Fish Meal (60\% Protein) & 10.0 \\
Corn Oil (without Preservatives) & 0.0 \\
Soy Oil (without Preservatives) & 2.5 \\
Dried Brewer's Yeast & 2.0 \\
Calcium Carbonate (USP) & 0.5 \\
Vitamin Premix & 0.25 \\
Mineral Premix & \\
Calcium Phosphate, Dibasic (USP) & 0.15 \\
Sodium Chloride & 1.25 \\
Choline Chloride (70\% Choline) & 0.5 \\
Dried Skim Milk & 0.10 \\
Dried Molasses & 5.00 \\
Corn Gluten Meal (60\% Protein) & 1.50 \\
Methionine & 3.00 \\
\hline USP Un & 0.0 \\
\hline
\end{tabular}

USP = United States Pharmacopeia

${ }^{a}$ Wheat middlings as carrier.

${ }^{\mathrm{b}}$ Calcium carbonate as carrier.

Table B-2. Vitamins and Minerals in NIH-07 Rat Ration

\begin{tabular}{lcl}
\hline & Amount $^{\mathbf{a}}$ & \multicolumn{1}{c}{ Source } \\
\hline Vitamins & & \\
Vitamin A & $6,062 \mathrm{IU}$ & Stabilized vitamin A palmitate or acetate \\
Vitamin D & $5,070 \mathrm{IU}$ & D-activated animal sterol \\
Vitamin K & $3.1 \mathrm{mg}$ & Menadione sodium bisulfite complex \\
Vitamin E & $22 \mathrm{IU}$ & $\alpha$-Tocopheryl acetate \\
Niacin & $33 \mathrm{mg}$ & - \\
Folic Acid & $2.4 \mathrm{mg}$ & - \\
\hline
\end{tabular}


Di(2-ethylhexyl) Phthalate, NTP TR 601

\begin{tabular}{lcl}
\hline & Amount $^{\mathrm{a}}$ & \multicolumn{1}{c}{ Source } \\
\hline d-Pantothenic Acid & $19.8 \mathrm{mg}$ & d-Calcium pantothenate \\
Riboflavin & $3.8 \mathrm{mg}$ & - \\
Thiamine & $11 \mathrm{mg}$ & Thiamine mononitrate \\
$\mathrm{B}_{12}$ & $50 \mu \mathrm{g}$ & - \\
Pyridoxine & $6.5 \mathrm{mg}$ & Pyridoxine hydrochloride \\
Biotin & $0.15 \mathrm{mg}$ & d-Biotin \\
Minerals & & \\
Iron & $132 \mathrm{mg}$ & Iron sulfate \\
Zinc & $18 \mathrm{mg}$ & Zinc oxide \\
Manganese & $66 \mathrm{mg}$ & Manganese oxide \\
Copper & $4.4 \mathrm{mg}$ & Copper sulfate \\
Iodine & $2.0 \mathrm{mg}$ & Calcium iodate \\
Cobalt & $0.44 \mathrm{mg}$ & Cobalt carbonate \\
\hline
\end{tabular}

aper kg of finished diet.

Table B-3. Nutrient Composition of NIH-07 Rat Ration

\begin{tabular}{lccc}
\hline \multicolumn{1}{c}{ Nutrient } & $\begin{array}{c}\text { Mean } \pm \text { Standard } \\
\text { Deviation }\end{array}$ & Range & Number of Samples \\
\hline Protein (\% by Weight) & $23.65 \pm 0.070$ & $23.6-23.7$ & 2 \\
Crude Fat (\% by Weight) & $5.15 \pm 0.212$ & $5.0-5.3$ & 2 \\
Crude Fiber (\% by Weight) & $3.29 \pm 0.042$ & $3.26-3.32$ & 2 \\
Ash (\% by Weight) & $6.015 \pm 0.092$ & $5.95-6.08$ & 2 \\
Amino Acids (\% of Total Diet) & & & 10 \\
Arginine & $1.380 \pm 0.06$ & $1.3-1.49$ & 10 \\
Cystine & $0.322 \pm 0.031$ & $0.274-0.372$ & 10 \\
Glycine & $1.150 \pm 0.070$ & $1.06-1.31$ & 10 \\
Histidine & $0.518 \pm 0.024$ & $0.497-0.553$ & 10 \\
Isoleucine & $0.984 \pm 0.024$ & $0.952-1.03$ & 10 \\
Leucine & $2.018 \pm 0.067$ & $1.93-2.13$ & 10 \\
Lysine & $1.243 \pm 0.051$ & $1.13-1.32$ & 10 \\
Methionine & $0.488 \pm 0.016$ & $0.468-0.515$ & 10 \\
Phenylalanine & $1.097 \pm 0.022$ & $1.07-1.12$ & 10 \\
Threonine & $0.918 \pm 0.031$ & $0.883-0.961$ & 10 \\
Tryptophan & $0.277 \pm 0.020$ & $0.265-0.326$ & 10 \\
Tyrosine & $0.860 \pm 0.037$ & $0.785-0.894$ & \\
Valine & $1.134 \pm 0.025$ & $1.11-1.17$ & \\
Essential Fatty Acids (\% of Total Diet) & $1.99-2.59$ & \\
Linoleic & $2.30 \pm 0.219$ & & \\
\hline & & & 10 \\
\hline
\end{tabular}


Di(2-ethylhexyl) Phthalate, NTP TR 601

\begin{tabular}{|c|c|c|c|}
\hline Nutrient & $\begin{array}{c}\text { Mean } \pm \text { Standard } \\
\text { Deviation }\end{array}$ & Range & Number of Samples \\
\hline Linolenic & $0.25 \pm 0.275$ & $0.217-0.296$ & 10 \\
\hline \multicolumn{4}{|l|}{ Vitamins } \\
\hline Vitamin A (IU/kg) & $6,020 \pm 65.05$ & $5,560-6,480$ & 2 \\
\hline$\alpha$-Tocopherol (ppm) & $6,704 \pm 21,045$ & $40.3-66,600$ & 10 \\
\hline Thiamine $(\mathrm{ppm})^{\mathrm{a}}$ & $14.2 \pm 0.566$ & $13.8-14.6$ & 2 \\
\hline Riboflavin (ppm) & $14.47 \pm 3.352$ & $10.0-19.8$ & 10 \\
\hline Niacin (ppm) & $99.33 \pm 8.235$ & $87.0-112.0$ & 10 \\
\hline Pantothenic Acid (ppm) & $44.38 \pm 3.806$ & $38.2-51.1$ & 10 \\
\hline Pyridoxine (ppm) ${ }^{\mathrm{a}}$ & $12.876 \pm 3.171$ & $9.63-19.7$ & 10 \\
\hline Folic Acid (ppm) & $2.482 \pm 0.487$ & $1.68-3.09$ & 10 \\
\hline Biotin (ppm) & $0.3283 \pm 0.172$ & $0.0-0.638$ & 10 \\
\hline $\mathrm{B}_{12}(\mathrm{ppb})$ & $49.4 \pm 6.83$ & $41.8-61.6$ & 10 \\
\hline Choline (as Chloride) (ppm) & $1,821.0 \pm 197.5$ & $1,570-2,200$ & 10 \\
\hline \multicolumn{4}{|l|}{ Minerals } \\
\hline Calcium (\%) & $1.004 \pm 0.008$ & $0.998-1.01$ & 2 \\
\hline Phosphorus (\%) & $0.910 \pm 0.002$ & $0.908-0.911$ & 2 \\
\hline Potassium (\%) & $0.830 \pm 0.036$ & $0.769-0.88$ & 10 \\
\hline Chloride (\%) & $0.652 \pm 0.106$ & $0.441-0.8$ & 10 \\
\hline Sodium (\%) & $0.378 \pm 0.46$ & $0.318-0.469$ & 10 \\
\hline Magnesium (\%) & $0.187 \pm 0.014$ & $0.17-0.218$ & 10 \\
\hline Iron (ppm) & $385.1 \pm 54.9$ & $276.0-469.0$ & 10 \\
\hline Manganese (ppm) & $90.81 \pm 7.566$ & $80.7-104.0$ & 10 \\
\hline Zinc (ppm) & $64.15 \pm 10.07$ & $52.4-89.2$ & 10 \\
\hline Copper (ppm) & $14.13 \pm 2.57$ & $11.9-21.1$ & 10 \\
\hline Iodine (ppm) & $1.811 \pm 0.992$ & $0.54-3.45$ & 10 \\
\hline Chromium (ppm) & $3.946 \pm 0.036$ & $3.89-4.0$ & 8 \\
\hline Cobalt (ppm) & $0.5155 \pm 0.267$ & $0.01-0.963$ & 10 \\
\hline
\end{tabular}

${ }^{\mathrm{a} A s}$ hydrochloride.

Table B-4. Contaminant Levels in NIH-07 Rat Ration

\begin{tabular}{lccc}
\hline & Mean \pm Standard Deviation & Range & $\begin{array}{c}\text { Number of } \\
\text { Samples }\end{array}$ \\
\hline Contaminants & & & \\
Arsenic (ppm) & $0.3865 \pm 0.013$ & $0.377-0.396$ & 2 \\
Cadmium (ppm) & $0.0875 \pm 0.004$ & $0.085-0.09$ & 2 \\
Lead (ppm) & $0.072 \pm 0.004$ & $0.069-0.074$ & 2 \\
Mercury (ppm) & $0.013 \pm 0.001$ & $0.012-0.014$ & 2 \\
Selenium (ppm) & $0.382 \pm 0.014$ & $0.372-0.392$ & 2 \\
\hline
\end{tabular}


Di(2-ethylhexyl) Phthalate, NTP TR 601

\begin{tabular}{|c|c|c|c|}
\hline & Mean \pm Standard Deviation & Range & $\begin{array}{c}\text { Number of } \\
\text { Samples }\end{array}$ \\
\hline Aflatoxins (ppb) ${ }^{\mathrm{a}}$ & 5 & - & 2 \\
\hline Nitrate Nitrogen $(\mathrm{ppm})^{\mathrm{b}}$ & $11.8 \pm 2.55$ & $10.0-13.6$ & 2 \\
\hline Nitrite Nitrogen (ppm) $)^{\mathrm{a}, \mathrm{b}}$ & $<0.61$ & - & 2 \\
\hline BHA (ppm) $)^{\mathrm{a}, \mathrm{c}}$ & $<1.0$ & - & 2 \\
\hline BHT (ppm) $)^{\mathrm{a}, \mathrm{c}}$ & $<1.0$ & - & 2 \\
\hline Aerobic Plate Count (CFU/gm) & $60 \pm 70.7$ & $10-110$ & 2 \\
\hline Coliform (MPN/gm) & $<3.0$ & - & 2 \\
\hline Escherichia coli (MPN/gm) & $<10$ & - & 2 \\
\hline Salmonella (MPN/gm) & Negative & - & 2 \\
\hline Total Nitrosamines (ppb) ${ }^{\mathrm{d}}$ & $5.5 \pm 1.768$ & $4.2-6.7$ & 2 \\
\hline N-Nitrosodimethylamine $(\mathrm{ppb})^{\mathrm{d}}$ & $4.5 \pm 1.768$ & $3.2-5.7$ & 2 \\
\hline N-Nitrosopyrrolidine $(\mathrm{ppb})^{\mathrm{d}}$ & $1 \pm 0.0$ & $1.0-1.0$ & 2 \\
\hline \multicolumn{4}{|l|}{ Pesticides (ppm) } \\
\hline$\alpha-\mathrm{BHC}^{\mathrm{a}}$ & $<0.01$ & - & 2 \\
\hline$\beta-\mathrm{BHC}^{\mathrm{a}}$ & $<0.02$ & - & 2 \\
\hline$\gamma-\mathrm{BHC}^{\mathrm{a}}$ & $<0.01$ & - & 2 \\
\hline$\delta-\mathrm{BHC}^{\mathrm{a}}$ & $<0.01$ & - & 2 \\
\hline Heptachlor $^{\mathrm{a}}$ & $<0.01$ & - & 2 \\
\hline Aldrin $^{\mathrm{a}}$ & $<0.01$ & - & 2 \\
\hline Heptachlor Epoxide ${ }^{a}$ & $<0.01$ & - & 2 \\
\hline $\mathrm{DDE}^{\mathrm{a}}$ & $<0.01$ & - & 2 \\
\hline $\mathrm{DDD}^{\mathrm{a}}$ & $<0.01$ & - & 2 \\
\hline $\mathrm{DDT}^{\mathrm{a}}$ & $<0.01$ & - & 2 \\
\hline $\mathrm{HCB}^{\mathrm{a}}$ & $<0.01$ & - & 2 \\
\hline Mirex $^{a}$ & $<0.01$ & - & 2 \\
\hline Methoxychlor ${ }^{\mathrm{a}}$ & $<0.05$ & - & 2 \\
\hline Dieldrin $^{\mathrm{a}}$ & $<0.01$ & - & 2 \\
\hline Endrin $^{\mathrm{a}}$ & $<0.01$ & - & 2 \\
\hline Telodrin $^{\mathrm{a}}$ & $<0.01$ & - & 2 \\
\hline Chlordane $^{\mathrm{a}}$ & $<0.05$ & - & 2 \\
\hline Toxaphene $^{\mathrm{a}}$ & $<0.10$ & - & 2 \\
\hline Estimated $\mathrm{PCBs}^{\mathrm{a}}$ & $<0.20$ & - & 2 \\
\hline Ronnel $^{\mathrm{a}}$ & $<0.01$ & - & 2 \\
\hline Ethion $^{\mathrm{a}}$ & $<0.02$ & - & 2 \\
\hline Trithion $^{\mathrm{a}}$ & $<0.05$ & - & 2 \\
\hline Diazinon $^{a}$ & $<0.10$ & - & 2 \\
\hline Methyl Chlorpyrifos & $<0.02$ & - & 2 \\
\hline Methyl Parathion ${ }^{\mathrm{a}}$ & $<0.02$ & - & 2 \\
\hline Ethyl Parathion ${ }^{\mathrm{a}}$ & $<0.02$ & - & 2 \\
\hline Malathion & $0.081 \pm 0.082$ & $0.024-0.139$ & 2 \\
\hline Endosulfan $\mathrm{I}^{\mathrm{a}}$ & $<0.01$ & - & 2 \\
\hline
\end{tabular}




\begin{tabular}{lccc}
\hline & Mean \pm Standard Deviation & Range & $\begin{array}{c}\text { Number of } \\
\text { Samples }\end{array}$ \\
\hline Endosulfan II $^{\mathrm{a}}$ & $<0.01$ & - & 2 \\
Endosulfane Sulfate $^{\mathrm{a}}$ & $<0.03$ & - & 2 \\
\hline
\end{tabular}

All samples were irradiated.

$\mathrm{BHA}=$ butylated hydroxyanisole; $\mathrm{BHT}=$ butylated hydroxytoluene; $\mathrm{CFU}=$ colony-forming units; MPN = most probable number; $\mathrm{BHC}=$ hexachlorocyclohexane or benzene hexachloride; $\mathrm{DDE}=$ dichlorodiphenyldichloroethylene;

$\mathrm{DDD}=$ dichlorodiphenyldichloroethane; DDT = dichlorodiphenyltrichloroethane; $\mathrm{HCB}=$ hexachlorobenzene;

$\mathrm{PCB}=$ polychlorinated biphenyl.

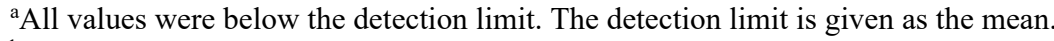

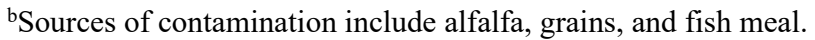

'Sources of contamination include soy oil and fish meal.

${ }^{\mathrm{d}}$ All values were corrected for percent recovery.

\section{B.2. NTP-2000 Feed}

Table B-5. Ingredients of NTP-2000 Rat Ration

\begin{tabular}{lc}
\hline \multicolumn{1}{c}{ Ingredients } & Percent by Weight \\
\hline Ground Hard Winter Wheat & 22.26 \\
Ground \#2 Yellow Shelled Corn & 22.18 \\
Wheat Middlings & 15.0 \\
Oat Hulls & 8.5 \\
Alfalfa Meal (Dehydrated, 17\% Protein) & 7.5 \\
Purified Cellulose & 5.5 \\
Soybean Meal (49\% Protein) & 5.0 \\
Fish Meal (60\% Protein) & 4.0 \\
Corn Oil (without Preservatives) & 3.0 \\
Soy Oil (without Preservatives) & 3.0 \\
Dried Brewer's Yeast & 1.0 \\
Calcium Carbonate (USP) & 0.9 \\
Vitamin Premix & 0.5 \\
Mineral Premix & 0.5 \\
Calcium Phosphate, Dibasic (USP) & 0.4 \\
Sodium Chloride & 0.3 \\
Choline Chloride (70\% Choline) & 0.26 \\
Methionine & 0.2 \\
\hline US $=$ Unt Stts & \\
\hline
\end{tabular}

USP = United States Pharmacopeia.

${ }^{a}$ Wheat middlings as carrier.

${ }^{\mathrm{b}}$ Calcium carbonate as carrier.

Table B-6. Vitamins and Minerals in NTP-2000 Rat Ration

\begin{tabular}{lll}
\hline & Amount $^{\mathbf{a}}$ & \multicolumn{1}{c}{ Source } \\
\hline Vitamins & & \\
Vitamin A & $4,000 \mathrm{IU}$ & Stabilized vitamin A palmitate or acetate \\
Vitamin D & $1,000 \mathrm{IU}$ & D-activated animal sterol \\
\hline
\end{tabular}


Di(2-ethylhexyl) Phthalate, NTP TR 601

\begin{tabular}{lcl}
\hline & Amount & \multicolumn{1}{c}{ Source } \\
\hline Vitamin K & $1.0 \mathrm{mg}$ & Menadione sodium bisulfite complex \\
$\alpha$-Tocopheryl Acetate & $100 \mathrm{IU}$ & - \\
Niacin & $23 \mathrm{mg}$ & - \\
Folic Acid & $1.1 \mathrm{mg}$ & - \\
d-Pantothenic Acid & $10 \mathrm{mg}$ & d-Calcium pantothenate \\
Riboflavin & $3.3 \mathrm{mg}$ & - \\
Thiamine & $4 \mathrm{mg}$ & Thiamine mononitrate \\
B $_{12}$ & $52 \mu \mathrm{g}$ & - \\
Pyridoxine & $6.3 \mathrm{mg}$ & Pyridoxine hydrochloride \\
Biotin & $0.2 \mathrm{mg}$ & d-Biotin \\
\hline Minerals & & \\
Magnesium & $514 \mathrm{mg}$ & Magnesium oxide \\
Iron & $35 \mathrm{mg}$ & Iron sulfate \\
Zinc & $12 \mathrm{mg}$ & Zinc oxide \\
Manganese & $10 \mathrm{mg}$ & Manganese oxide \\
Copper & $2.0 \mathrm{mg}$ & Copper sulfate \\
Iodine & $0.2 \mathrm{mg}$ & Calcium iodate \\
Chromium & $0.2 \mathrm{mg}$ & Chromium acetate \\
\hline
\end{tabular}

${ }^{\mathrm{a} P e r ~ k g}$ of finished diet.

Table B-7. Nutrient Composition of NTP-2000 Rat Ration

\begin{tabular}{lccc}
\hline \multicolumn{1}{c}{ Nutrient } & $\begin{array}{c}\text { Mean } \pm \text { Standard } \\
\text { Deviation }\end{array}$ & Range & Number of Samples \\
\hline Protein (\% by Weight) & $14.78 \pm 0.543$ & $13.9-16.8$ & 28 \\
Crude Fat (\% by Weight) & $8.63 \pm 0.387$ & $8.0-9.7$ & 28 \\
Crude Fiber (\% by Weight) & $9.37 \pm 0.534$ & $7.49-10.1$ & 28 \\
Ash (\% by Weight) & $5.23 \pm 1.767$ & $4.6-14.2$ & 28 \\
\hline Amino Acids (\% of Total Diet) & & & 29 \\
Arginine & $0.805 \pm 0.075$ & $0.67-0.97$ & 29 \\
Cystine & $0.220 \pm 0.021$ & $0.15-0.25$ & 29 \\
Glycine & $0.702 \pm 0.038$ & $0.62-0.80$ & 29 \\
Histidine & $0.342 \pm 0.070$ & $0.27-0.68$ & 29 \\
Isoleucine & $0.549 \pm 0.040$ & $0.43-0.66$ & 29 \\
Leucine & $1.100 \pm 0.063$ & $0.96-1.24$ & 29 \\
Lysine & $0.700 \pm 0.104$ & $0.31-0.86$ & 29 \\
Methionine & $0.409 \pm 0.042$ & $0.26-0.49$ & 29 \\
Phenylalanine & $0.623 \pm 0.047$ & $0.471-0.72$ & \\
\hline
\end{tabular}


Di(2-ethylhexyl) Phthalate, NTP TR 601

\begin{tabular}{|c|c|c|c|}
\hline Nutrient & $\begin{array}{c}\text { Mean } \pm \text { Standard } \\
\text { Deviation }\end{array}$ & Range & Number of Samples \\
\hline Threonine & $0.513 \pm 0.041$ & $0.43-0.61$ & 29 \\
\hline Tryptophan & $0.155 \pm 0.027$ & $0.11-0.2$ & 29 \\
\hline Tyrosine & $0.422 \pm 0.066$ & $0.28-0.54$ & 29 \\
\hline Valine & $0.666 \pm 0.040$ & $0.55-0.73$ & 29 \\
\hline \multicolumn{4}{|c|}{ Essential Fatty Acids (\% of Total Diet) } \\
\hline Linoleic & $3.94 \pm 0.235$ & $3.49-4.55$ & 29 \\
\hline Linolenic & $0.30 \pm 0.064$ & $0.005-0.368$ & 29 \\
\hline \multicolumn{4}{|l|}{ Vitamins } \\
\hline Vitamin A (IU/kg) & $3,886 \pm 81.3$ & $2,030-5,290$ & 28 \\
\hline$\alpha$-Tocopherol (ppm) & $2,456 \pm 12,817$ & $13.6-69,100$ & 29 \\
\hline Thiamine $(\mathrm{ppm})^{\mathrm{a}}$ & $7.96 \pm 0.484$ & $3.9-11.1$ & 28 \\
\hline Riboflavin (ppm) & $8.17 \pm 2.841$ & $4.2-17.5$ & 29 \\
\hline Niacin (ppm) & $78.66 \pm 8.11$ & $66.4-98.2$ & 29 \\
\hline Pantothenic Acid (ppm) & $26.42 \pm 11.05$ & $17.4-81.0$ & 29 \\
\hline Pyridoxine $(\mathrm{ppm})^{\mathrm{a}}$ & $9.75 \pm 2.045$ & $6.44-14.3$ & 29 \\
\hline Folic Acid (ppm) & $1.58 \pm 0.43$ & $1.15-3.27$ & 29 \\
\hline Biotin (ppm) & $0.323 \pm 0.093$ & $0.2-0.704$ & 29 \\
\hline $\mathrm{B}_{12}(\mathrm{ppb})$ & $50.41 \pm 34.89$ & $18.3-174$ & 29 \\
\hline Choline (as Chloride) (ppm) & $2,593 \pm 633.8$ & $1,160-3,790$ & 29 \\
\hline \multicolumn{4}{|l|}{ Minerals } \\
\hline Calcium (\%) & $0.905 \pm 0.041$ & $0.831-1.03$ & 28 \\
\hline Phosphorus (\%) & $0.540 \pm 0.097$ & $0.053-0.60$ & 28 \\
\hline Potassium (\%) & $0.668 \pm 0.029$ & $0.626-0.733$ & 29 \\
\hline Chloride (\%) & $0.392 \pm 0.044$ & $0.3-0.517$ & 29 \\
\hline Sodium (\%) & $0.195 \pm 0.027$ & $0.16-0.283$ & 29 \\
\hline Magnesium (\%) & $0.217 \pm 0.054$ & $0.185-0.49$ & 29 \\
\hline Iron (ppm) & $191.6 \pm 36.18$ & $135-311$ & 29 \\
\hline Manganese (ppm) & $50.11 \pm 9.42$ & $21-73.1$ & 29 \\
\hline Zinc (ppm) & $57.3 \pm 25.54$ & $43.3-184$ & 29 \\
\hline Copper (ppm) & $7.57 \pm 2.49$ & $3.21-16.3$ & 29 \\
\hline Iodine (ppm) & $0.513 \pm 0.221$ & $0-0.972$ & 29 \\
\hline Chromium (ppm) & $1.02 \pm 1.04$ & $0.33-3.97$ & 28 \\
\hline Cobalt (ppm) & $0.222 \pm 0.152$ & $0.0857-0.864$ & 27 \\
\hline
\end{tabular}

${ }^{a}$ As hydrochloride. 
Di(2-ethylhexyl) Phthalate, NTP TR 601

Table B-8. Contaminant Levels in NTP-2000 Rat Ration

\begin{tabular}{|c|c|c|c|}
\hline & Mean \pm Standard Deviation & Range & $\begin{array}{c}\text { Number of } \\
\text { Samples }\end{array}$ \\
\hline \multicolumn{4}{|l|}{ Contaminants } \\
\hline Arsenic (ppm) & $0.2 \pm 0.048$ & $0.147-0.383$ & 28 \\
\hline Cadmium (ppm) & $0.051 \pm 0.008$ & $0.038-0.082$ & 28 \\
\hline Lead (ppm) & $0.144 \pm 0.110$ & $0.064-0.474$ & 28 \\
\hline Mercury (ppm) & $0.0115 \pm 0.004$ & $0.01-0.03$ & 28 \\
\hline Selenium (ppm) & $0.161 \pm 0.034$ & $0.029-0.242$ & 28 \\
\hline Aflatoxins $(\mathrm{ppb})^{\mathrm{a}}$ & $<5.0$ & - & 28 \\
\hline Nitrate Nitrogen $(\mathrm{ppm})^{\mathrm{b}}$ & $15.7 \pm 5.98$ & $10.0-35.1$ & 28 \\
\hline Nitrite Nitrogen $(\mathrm{ppm})^{\mathrm{a}, \mathrm{b}}$ & $<0.61$ & - & 28 \\
\hline BHA $(p p m)^{\mathrm{a}, \mathrm{c}}$ & $<1.00$ & - & 28 \\
\hline BHT (ppm) $)^{\mathrm{a}, \mathrm{c}}$ & $<1.00$ & - & 28 \\
\hline Aerobic Plate Count (CFU/gm) & $<10.0$ & - & 28 \\
\hline Coliform (MPN/gm) & $<3$ & - & 28 \\
\hline Escherichia coli (MPN/gm) & $<10.0$ & - & 28 \\
\hline Salmonella (MPN/gm) & Negative & - & 28 \\
\hline Total Nitrosamines $(\mathrm{ppb})^{\mathrm{d}}$ & $10.5 \pm 6.01$ & $1.5-24.5$ & 28 \\
\hline N-Ndimethylamine $(\mathrm{ppb})^{\mathrm{d}}$ & $2.2 \pm 1.50$ & $0-6.6$ & 28 \\
\hline N-Npyrrolidine $(\mathrm{ppb})^{\mathrm{d}}$ & $8.3 \pm 5.47$ & $1.4-20.0$ & 28 \\
\hline \multicolumn{4}{|l|}{ Pesticides (ppm) } \\
\hline$\alpha-\mathrm{BHC}^{\mathrm{a}}$ & $<0.01$ & - & 28 \\
\hline$\beta-B H C^{a}$ & $<0.02$ & - & 28 \\
\hline$\gamma-\mathrm{BHC}^{\mathrm{a}}$ & $<0.01$ & - & 28 \\
\hline$\delta-\mathrm{BHC}^{\mathrm{a}}$ & $<0.01$ & - & 28 \\
\hline Heptachlor $^{\mathrm{a}}$ & $<0.01$ & - & 28 \\
\hline Aldrin $^{\mathrm{a}}$ & $<0.01$ & - & 28 \\
\hline Heptachlor Epoxide & $<0.01$ & - & 28 \\
\hline $\mathrm{DDE}^{\mathrm{a}}$ & $<0.01$ & - & 28 \\
\hline $\mathrm{DDD}^{\mathrm{a}}$ & $<0.01$ & - & 28 \\
\hline $\mathrm{DDT}^{\mathrm{a}}$ & $<0.01$ & - & 28 \\
\hline $\mathrm{HCB}^{\mathrm{a}}$ & $<0.01$ & - & 28 \\
\hline Mirex $^{\mathrm{a}}$ & $<0.01$ & - & 28 \\
\hline Methoxychlor ${ }^{\mathrm{a}}$ & $<0.05$ & - & 28 \\
\hline Dieldrin $^{\mathrm{a}}$ & $<0.01$ & - & 28 \\
\hline Endrin $^{\mathrm{a}}$ & $<0.01$ & - & 28 \\
\hline Telodrin $^{\mathrm{a}}$ & $<0.01$ & - & 28 \\
\hline Chlordane $^{a}$ & $<0.05$ & - & 28 \\
\hline
\end{tabular}




\begin{tabular}{lccc}
\hline & Mean \pm Standard Deviation & Range & $\begin{array}{c}\text { Number of } \\
\text { Samples }\end{array}$ \\
\hline Toxaphene $^{\mathrm{a}}$ & $<0.10$ & - & 28 \\
Estimated PCBs $^{\mathrm{a}}$ & $<0.20$ & - & 28 \\
Ronnel $^{\mathrm{a}}$ & $<0.01$ & - & 28 \\
Ethion $^{\mathrm{a}}$ & $<0.02$ & - & 28 \\
Trithion $^{\mathrm{a}}$ & $<0.05$ & - & 28 \\
Diazinon $^{\mathrm{a}}$ & $<0.10$ & - & 28 \\
Methyl Chlorpyrifos $^{\mathrm{a}}$ & $0.092 \pm 0.075$ & - & 28 \\
Methyl Parathion $^{\mathrm{a}}$ & $<0.02$ & - & 28 \\
Ethyl Parathion $^{\mathrm{a}}$ & $<0.02$ & $0.02-0.297$ & 28 \\
Malathion & $0.071 \pm 0.07$ & - & 28 \\
Endosulfan I $^{\mathrm{a}}$ & $<0.01$ & - & 28 \\
Endosulfan II $^{\mathrm{a}}$ & $<0.01$ & - & 28 \\
Endosulfane Sulfate $^{\mathrm{a}}$ & $<0.03$ & & \\
\hline Allsps & & - & 28 \\
\hline
\end{tabular}

All samples were irradiated.

$\mathrm{BHA}=$ butylated hydroxyanisole; $\mathrm{BHT}=$ butylated hydroxytoluene; $\mathrm{CFU}=$ colony-forming units; $\mathrm{MPN}=$ most probable number; $\mathrm{BHC}=$ hexachlorocyclohexane or benzene hexachloride; DDE = dichlorodiphenyldichloroethylene;

$\mathrm{DDD}=$ dichlorodiphenyldichloroethane; DDT = dichlorodiphenyltrichloroethane; $\mathrm{HCB}=$ hexachlorobenzene;

$\mathrm{PCB}=$ polychlorinated biphenyl.

${ }^{a}$ All values were below the detection limit. The detection limit is given as the mean.

bources of contamination include alfalfa, grains, and fish meal.

'Sources of contamination include soy oil and fish meal.

${ }^{\mathrm{d} A}$ All values were corrected for percent recovery. 


\section{Appendix C. Sentinel Animal Program \\ Table of Contents}

C.1. Methods

C-2

C.2. Results

\section{Tables}

Table C-1. Methods and Results for Sentinel Animal Testing in Male and Female Rats (Study 1)

Table C-2. Methods and Results for Sentinel Animal Testing in Male and Female Rats (Study 2) ..C-4 


\section{C.1. Methods}

Rodents used in the National Toxicology Program are produced in optimally clean facilities to eliminate potential pathogens that might affect study results. The Sentinel Animal Program is part of the periodic monitoring of animal health that occurs during the toxicological evaluation of test compounds. Under this program, the disease state of the rodents is monitored via sera or feces from extra (sentinel) or exposed animals in the study rooms. The sentinel animals and the study animals are subject to identical environmental conditions. Furthermore, the sentinel animals are from the same production source and weanling groups as the animals used for the studies of test compounds.

For these toxicology and carcinogenesis studies, blood samples were collected from each sentinel animal, allowed to clot, and the serum was separated. Additionally, fecal samples were collected and tested for endoparasites and Helicobacter species. All samples were processed appropriately with serology and Helicobacter testing performed by IDEXX BioResearch (formerly Rodent Animal Diagnostic Laboratory [RADIL], University of Missouri), Columbia, $\mathrm{MO}$, for determination of the presence of pathogens. Evaluation for endo- and ectoparasites was performed in-house by the testing laboratory.

The laboratory methods and agents for which testing was performed are tabulated in Table C-1, Table C-2 below; the times at which samples were collected during the studies are also listed.

\section{C.2. Results}

\section{C.2.1. Perinatal and Postweaning Study (Study 1)}

Rats: Positive for endoparasites, pinworms (Syphacia spp.). All other test results were negative.

\section{C.2.2. Postweaning-only Study (Study 2)}

Rats: Positive for endoparasites, pinworms (Syphacia spp.). All other test results were negative. 
Table C-1. Methods and Results for Sentinel Animal Testing in Male and Female Rats (Study 1)

\begin{tabular}{|c|c|c|c|c|c|c|c|}
\hline Collection Time Points & Quarantine $^{\mathbf{a}}$ & 3.5 Weeks ${ }^{b}$ & $\begin{array}{l}4 \text { Weeks Post- } \\
\text { Study Start }\end{array}$ & 6 Months & 12 Months & 18 Months & End of Study \\
\hline Number Examined (Males/Females) & $0 / 10$ & $0 / 10$ & $5 / 5$ & $5 / 5$ & $6 / 6^{\mathrm{d}}$ & $5 / 6^{\mathrm{e}}$ & $5 / 5$ \\
\hline \multicolumn{8}{|l|}{ Method/Test } \\
\hline \multicolumn{8}{|l|}{ Multiplex Fluorescent Immunoassay (MFI) } \\
\hline Kilham rat virus (KRV) & - & - & - & - & - & - & - \\
\hline Mycoplasma pulmonis & - & - & - & - & - & - & - \\
\hline Parvo NS-1 & - & - & - & - & - & - & - \\
\hline Pneumonia virus of mice (PVM) & - & - & - & - & - & - & - \\
\hline Rat coronavirus/sialodacryoadenitis virus (RCV/SDA) & - & - & - & - & - & - & - \\
\hline Rat minute virus (RMV) & - & - & - & - & - & - & - \\
\hline Rat parvo virus (RPV) & - & - & - & - & - & - & - \\
\hline Rat theilovirus (RTV) & - & - & - & - & - & - & - \\
\hline Sendai & - & - & - & - & - & - & - \\
\hline Theiler's murine encephalomyelitis virus (TMEV) & - & - & - & - & - & - & - \\
\hline Toolan's H-1 & - & - & - & - & - & - & - \\
\hline \multicolumn{8}{|l|}{ Immunofluorescence Assay (IFA) } \\
\hline Pneumocystis carinii & NT & NT & NT & - & NT & NT & NT \\
\hline \multicolumn{8}{|l|}{ In-house Evaluation } \\
\hline $\begin{array}{l}\text { Endoparasite evaluation (evaluation of } \\
\text { cecal content) }\end{array}$ & - & NT & NT & NT & + & + & NT \\
\hline $\begin{array}{l}\text { Ectoparasite evaluation (evaluation of } \\
\text { perianal surface) }\end{array}$ & - & NT & NT & NT & - & - & NT \\
\hline $\begin{array}{l}-=\text { negative; }+=\text { positive; } \mathrm{NT}=\text { not tested. } \\
\text { a Age-matched nonpregnant females. } \\
\text { bTime-mated females that did not have a litter; } 3.5 \text { weeks } \\
{ }^{\mathrm{b}} \mathrm{F}_{1} \text { sentinel animals tested } 4 \text { weeks after } 2 \text {-year study start } \\
\text { dIncludes samples from one male and one female euthaniz } \\
\text { Includes samples from one female euthanized as moribun }\end{array}$ & $\begin{array}{l}\text { er arrival. } \\
\text { as moribund. }\end{array}$ & & & & & & \\
\hline
\end{tabular}


Table C-2. Methods and Results for Sentinel Animal Testing in Male and Female Rats (Study 2)

\begin{tabular}{|c|c|c|c|c|c|}
\hline Collection Time Points & 4 Weeks $^{\mathrm{a}}$ & 6 Months & 12 Months & 18 Months & End of Study \\
\hline Number Examined (Males/Females) & $5 / 5$ & $5 / 5$ & $5 / 5$ & $5 / 5$ & $5 / 5$ \\
\hline \multicolumn{6}{|l|}{ Method/Test } \\
\hline \multicolumn{6}{|l|}{ Multiplex Fluorescent Immunoassay (MFI) } \\
\hline Kilham rat virus (KRV) & - & - & - & - & - \\
\hline Mycoplasma pulmonis & - & - & - & - & - \\
\hline Parvo NS-1 & - & - & - & - & - \\
\hline Pneumonia virus of mice (PVM) & - & - & - & - & - \\
\hline $\begin{array}{l}\text { Rat coronavirus/sialodacryoadenitis } \\
\text { virus (RCV/SDA) }\end{array}$ & - & - & - & - & - \\
\hline Rat minute virus (RMV) & - & - & - & - & - \\
\hline Rat parvo virus (RPV) & - & - & - & - & - \\
\hline Rat theilovirus (RTV) & - & - & - & - & - \\
\hline Sendai & - & - & - & - & - \\
\hline $\begin{array}{l}\text { Theiler's murine encephalomyelitis } \\
\text { virus (TMEV) }\end{array}$ & - & - & - & - & - \\
\hline Toolan's H-1 & - & - & - & - & - \\
\hline \multicolumn{6}{|l|}{ Immunofluorescence Assay (IFA) } \\
\hline Kilham rat virus (KRV) & - & NT & NT & NT & NT \\
\hline Pneumocystis carinii & NT & - & NT & NT & NT \\
\hline Number Examined (Males/Females) & $0 / 0$ & $0 / 0$ & $6 / 5$ & $6 / 5$ & $0 / 0$ \\
\hline \multicolumn{6}{|l|}{ Method/Test } \\
\hline \multicolumn{6}{|l|}{ In-house Evaluation } \\
\hline $\begin{array}{l}\text { Endoparasite evaluation } \\
\text { (evaluation of cecal content) }\end{array}$ & NT & NT & + & + & NT \\
\hline $\begin{array}{l}\text { Ectoparasite evaluation } \\
\text { (evaluation of perianal surface) }\end{array}$ & NT & NT & + & + & NT \\
\hline
\end{tabular}




\section{Appendix D. Genetic Toxicology}

\section{Table of Contents}

D.1. Rodent Chromosome Aberrations Test..................................................................... D-2

D.2. In Vivo Micronucleus Test ................................................................................. D

\section{Tables}

Table D-1. Chromosomal Aberrations in Mice Exposed to Di(2-ethylhexyl) Phthalate in Feed for 14 Days ..................................................................................... D-2

Table D-2. Frequency of Micronuclei in the Bone Marrow of Female B6C3F1 Mice Exposed to Di(2-ethylhexyl) Phthalate in Feed for 14 Days

Table D-3. Frequency of Micronuclei in Peripheral Blood Erythrocytes of Male and Female TgAC (FVB/N) Mice Exposed to Di(2-ethylhexyl) Phthalate in Feed for 26 Weeks

Table D-4. Frequency of Micronuclei in Peripheral Blood Erythrocytes of Male and Female TgAC (FVB/N) Mice Following Dermal Application of Di(2-ethylhexyl) Phthalate for 26 Weeks 


\section{D.1. Rodent Chromosome Aberrations Test}

\section{D.1.1. Methods}

Female B6C3F1 mice (four animals per exposure group) were exposed to di(2-ethylhexyl) phthalate (DEHP) $(0,3,000,6,000$, or 12,000 ppm) in dosed feed for 14 days. The animals were subcutaneously implanted with a bromodeoxyuridine (BrdU) tablet ${ }^{241} 18$ hours before the scheduled harvest to allow selection of the appropriate cell population for scoring. ${ }^{242 ; 243}$ Chromosomal aberrations induced by test article administration are present in maximum number at the first metaphase following exposure; they decline in number during subsequent nuclear divisions due to cell death. Two hours before sacrifice, the animals received an intraperitoneal injection of colchicine in saline. The animals were euthanized 18 hours after BrdU dosing. One or both femurs were removed, and the marrow was flushed out with phosphate-buffered saline $(\mathrm{pH}$ 7.0). Cells were treated with a hypotonic salt solution, fixed, and dropped onto chilled slides. After a 24-hour drying period, the slides were stained (with a modified fluorescence-plusGiemsa technique) and scored.

Fifty first-division metaphase cells were scored from each animal. Responses were evaluated as the percentage of aberrant metaphase cells, excluding gaps. The data were analyzed by a trend test ${ }^{244}$ with $\mathrm{p} \leq 0.025$ considered to be significant. Pairwise comparisons of each exposure group to the corresponding solvent control group were considered significant for $p \leq 0.025 / 3$ (the number of DEHP-exposed groups).

\section{D.1.2. Results}

In vivo, no significant increases were observed in chromosomal aberrations in bone marrow cells of female B6C3F1 mice following administration of DEHP (3,000-12,000 ppm) in dosed feed for 14 days (Table D-1).

Table D-1. Chromosomal Aberrations in Mice Exposed to Di(2-ethylhexyl) Phthalate in Feed for 14 Days $^{\mathrm{a}, \mathrm{b}}$

\begin{tabular}{|c|c|c|c|}
\hline & $\mathbf{n}$ & Percent Cells with Aberrations & P Value (Pairwise) \\
\hline \multicolumn{4}{|l|}{ DEHP (ppm) } \\
\hline 0 & 4 & $1.25 \pm 0.48$ & 0.0000 \\
\hline 3,000 & 4 & $2.75 \pm 1.11$ & 0.0649 \\
\hline 6,000 & 4 & $0.50 \pm 0.29$ & 0.8726 \\
\hline 12,000 & 4 & $1.50 \pm 0.87$ & 0.3807 \\
\hline Trend $^{\mathrm{c}}$ & & $\mathrm{p}=0.6617$ & \\
\hline
\end{tabular}

DEHP $=$ di(2-ethylhexyl) phthalate.

aData are presented as the mean frequency of aberrant cells \pm standard error. Gaps were not included in the calculation of the mean frequency of chromosomally aberrant cells.

${ }^{b}$ Pairwise comparisons to the vehicle control group performed using a t-test ( $\mathrm{p} \leq 0.025 /$ number of exposed groups).

${ }^{c}$ Exposure-related trends evaluated using the Cochran-Armitage trend test $(\mathrm{p} \leq 0.025)$. 


\section{D.2. In Vivo Micronucleus Test}

\section{D.2.1. Methods}

\section{D.2.1.1. Bone Marrow}

Female B6C3F1 mice (10 animals per exposure group) were exposed to DEHP $(0,3,000,6,000$, or 12,000 ppm) in dosed feed for 14 days. Bone marrow smears were prepared from cells obtained from the femurs as described for the chromosomal aberrations assay. Air-dried smears were fixed and stained with acridine orange; 1,000 polychromatic erythrocytes (PCEs) were scored per animal for the frequency of micronucleated cells.

The results were tabulated as the mean of the pooled results from all animals within an exposure group \pm the standard error of the mean. The frequency of micronucleated cells among PCEs was analyzed for positive trend over the four exposure groups using a one-tailed Cochran-Armitage trend test, followed by pairwise comparisons between each exposed group and the concurrent control group. In the presence of excess binomial variation, as detected by a binomial dispersion test, the binomial variance of the Cochran-Armitage test was adjusted upward in proportion to the excess variation. For a test to be considered positive, the trend test $p$ value $\leq 0.025$ is required, along with at least one significant exposure group $(\mathrm{p} \leq 0.025$ divided by the number of exposed groups). Ultimately, the final call is determined by scientific staff after considering the results of statistical analyses, the reproducibility of any effects observed, and the magnitudes of those effects.

\section{D.2.1.2. Peripheral Blood}

A detailed discussion of this assay is presented by MacGregor et al. ${ }^{245}$ Two 26-week exposure protocols were used. In the first study, male and female $\mathrm{TgAC}(\mathrm{FVB} / \mathrm{N})$ transgenic mice were exposed to DEHP $(0,1,500,3,000$, or $6,000 \mathrm{ppm})$ in dosed feed. In the second study, male and female $\operatorname{TgAC}(\mathrm{FVB} / \mathrm{N})$ transgenic mice were exposed to $\operatorname{DEHP}(0,100,200$, or $400 \mathrm{mg} / \mathrm{kg})$ via dermal application. In both studies, at the end of the 26-week exposure period, peripheral blood samples were obtained. Smears were immediately prepared and fixed in absolute methanol. The methanol-fixed slides were stained with acridine orange and coded. Slides were scanned to determine the frequency of micronuclei in 1,000 normochromatic erythrocytes (NCEs) and 1,000 PCEs per animal.

The results were tabulated as the mean of the pooled results from all animals within an exposure group \pm the standard error of the mean. The frequency of micronucleated NCEs was analyzed as described above for the bone marrow micronucleus test.

\section{D.2.1.3. Evaluation Protocol}

These are the basic guidelines for arriving at an overall assay result for assays performed by the National Toxicology Program. Statistical as well as biological factors are considered. For an individual assay, the statistical procedures for data analysis have been described in the preceding protocols. There have been instances, however, in which multiple aliquots of a chemical were tested in the same assay, and differing results were obtained among aliquots and/or among laboratories. Results from more than one aliquot or from more than one laboratory are not simply combined into an overall result. Rather, all the data are critically evaluated, particularly with regard to pertinent protocol variations, in determining the weight of evidence for an overall 
conclusion of chemical activity in an assay. For in vitro assays conducted with and without exogenous metabolic activation, results from each testing condition are evaluated and reported separately. The summary table in the abstract of this Technical Report presents a result that represents a scientific judgement of the overall evidence for activity of the chemical in an assay.

\section{D.2.2. Results}

DEHP was tested in three independent erythrocyte micronucleus tests and produced varying results (Table D-2, Table D-3, Table D-4). In an in vivo bone marrow micronucleus test, B6C3F1 female mice were exposed to DEHP (3,000-12,000 ppm) in dosed feed for 14 days; results were judged to be equivocal (Table D-2). In a second test, DEHP (1,500-6,000 ppm) induced an equivocal response in male $\mathrm{TgAC}(\mathrm{FVB} / \mathrm{N})$ mice and a positive response in female $\operatorname{TgAC}(\mathrm{FVB} / \mathrm{N})$ mice following exposure via dosed feed for 26 weeks (Table D-3). Another 26-week exposure test in TgAC (FVB/N) mice using dermal application of DEHP (100$400 \mathrm{mg} / \mathrm{kg}$ ) produced negative results in both male and female mice (Table D-4).

Table D-2. Frequency of Micronuclei in the Bone Marrow of Female B6C3F1 Mice Exposed to Di(2-ethylhexyl) Phthalate in Feed for 14 Days

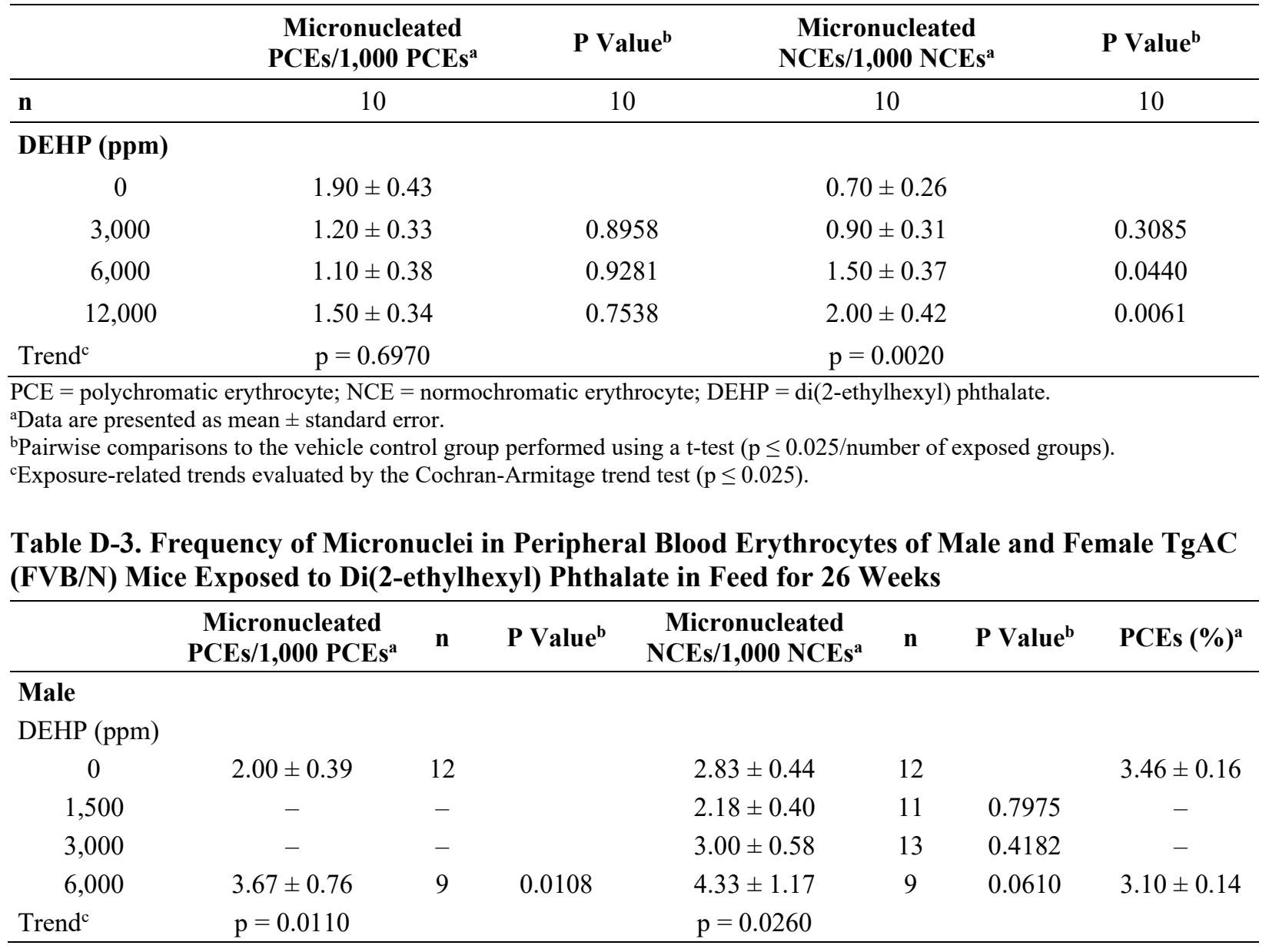




\begin{tabular}{lccccccc}
\hline & $\begin{array}{c}\text { Micronucleated } \\
\text { PCEs/1,000 PCEs }^{\mathbf{a}}\end{array}$ & $\mathbf{n}$ & P Value $^{\mathbf{b}}$ & $\begin{array}{c}\text { Micronucleated } \\
\text { NCEs/1,000 NCEs }\end{array}$ & $\mathbf{n}^{\mathbf{a}}$ & P Value $^{\mathbf{b}}$ & PCEs (\%) $^{\mathbf{a}}$ \\
\hline Female & & & & & & & \\
DEHP (ppm) & & & & & & & \\
$\quad 0$ & $2.50 \pm 0.56$ & 10 & & $1.40 \pm 0.27$ & 10 & & $3.47 \pm 0.63$ \\
1,500 & - & - & & $2.31 \pm 0.38$ & 13 & 0.0592 & - \\
3,000 & - & - & & $1.50 \pm 0.43$ & 6 & 0.4358 & - \\
$\quad 6,000$ & $1.27 \pm 0.33$ & 11 & 0.9804 & $3.27 \pm 0.51$ & 11 & 0.0027 & $3.18 \pm 0.18$ \\
Trend $^{\mathrm{c}}$ & $\mathrm{p}=0.9800$ & & & $\mathrm{p}=0.0040$ & & & \\
\hline
\end{tabular}

$\mathrm{PCE}=$ polychromatic erythrocyte; $\mathrm{NCE}=$ normochromatic erythrocyte; DEHP $=$ di(2-ethylhexyl) phthalate.

${ }^{a}$ Data are presented as mean \pm standard error.

${ }^{b}$ Pairwise comparisons to the vehicle control group performed using a t-test ( $\mathrm{p} \leq 0.025 /$ number of exposed groups).

${ }^{\mathrm{c}}$ Exposure-related trends evaluated by the Cochran-Armitage trend test $(\mathrm{p} \leq 0.025)$.

Table D-4. Frequency of Micronuclei in Peripheral Blood Erythrocytes of Male and Female TgAC (FVB/N) Mice Following Dermal Application of Di(2-ethylhexyl) Phthalate for 26 Weeks

\begin{tabular}{|c|c|c|c|c|c|c|c|}
\hline & $\begin{array}{l}\text { Micronucleated } \\
\text { PCEs/1,000 PCEs }{ }^{\mathrm{a}}\end{array}$ & $\mathbf{n}$ & P Value ${ }^{b}$ & $\begin{array}{c}\text { Micronucleated } \\
\text { NCEs/1,000 NCEs }{ }^{\mathrm{a}}\end{array}$ & $\mathbf{n}$ & P Value ${ }^{b}$ & $\operatorname{PCEs}(\%)^{\mathrm{a}}$ \\
\hline \multicolumn{8}{|l|}{ Male } \\
\hline \multicolumn{8}{|c|}{ DEHP (mg/kg) } \\
\hline 0 & $2.45 \pm 0.51$ & 11 & & $3.45 \pm 0.59$ & 11 & & $4.20 \pm 0.34$ \\
\hline 100 & - & - & & $3.17 \pm 0.34$ & 12 & 0.6480 & - \\
\hline 200 & - & - & & $3.36 \pm 0.37$ & 14 & 0.5522 & - \\
\hline 400 & $3.86 \pm 0.67$ & 7 & 0.0467 & $2.71 \pm 0.68$ & 7 & 0.8056 & $3.21 \pm 0.28$ \\
\hline Trend $^{\mathrm{c}}$ & $\mathrm{p}=0.0470$ & & & $\mathrm{p}=0.7700$ & & & \\
\hline \multicolumn{8}{|l|}{ Female } \\
\hline \multicolumn{8}{|c|}{ DEHP (mg/kg) } \\
\hline 0 & $2.55 \pm 0.47$ & 11 & & $1.64 \pm 0.36$ & 11 & & $4.86 \pm 0.34$ \\
\hline 100 & - & - & & $1.91 \pm 0.41$ & 11 & 0.3153 & - \\
\hline 200 & - & - & & $2.36 \pm 0.49$ & 11 & 0.1137 & - \\
\hline 400 & $4.09 \pm 0.56$ & 11 & 0.0231 & $2.55 \pm 0.45$ & 11 & 0.0700 & $4.24 \pm 0.19$ \\
\hline Trend $^{\mathrm{c}}$ & $\mathrm{p}=0.0230$ & & & $\mathrm{p}=0.0590$ & & & \\
\hline
\end{tabular}




\section{Appendix E. Mono(2-ethylhexyl) Phthalate Internal Dose Assessment}

\section{Table of Contents}

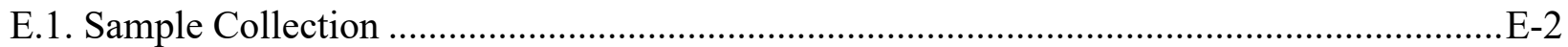

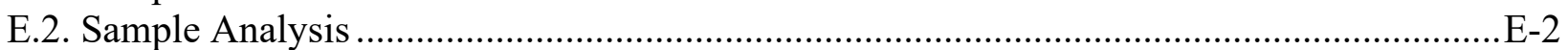

E.3. Instrumentation and Quantitation .......................................................................... E-2

\section{Tables}

Table E-1. Analytical Method Validation and Stability Data for Mono(2-ethylhexyl)

Phthalate in Plasma, Amniotic Fluid, and Fetal Homogenate 


\section{E.1. Sample Collection}

Select dams and their litters were removed on gestation day (GD) 18 to quantify mono(2-ethylhexyl) phthalate (MEHP) plasma and tissue concentrations. On GD 18, blood was collected from the retroorbital sinus of randomly selected dams ( $n=5$ per exposure group). Blood samples were collected in tubes containing K3 EDTA (tripotassium ethylene diamine tetraacetic acid), centrifuged, and the plasma harvested. Amniotic fluid was collected and pooled by dam, and each dam's fetuses were collected and pooled by litter. All samples were flash frozen in liquid nitrogen and stored frozen at approximately $-20^{\circ} \mathrm{C}$ before shipment to RTI International (Research Triangle Park, NC) for analysis.

\section{E.2. Sample Analysis}

MEHP, a metabolite of di(2-ethylhexyl) phthalate, was measured in dam plasma, amniotic fluid, and fetal homogenate using a validated analytical method. The analyte stability in each matrix was also confirmed; corresponding data are given in Table E-1.

MEHP stock solutions were prepared in methanol at $0.5 \mathrm{mg} / \mathrm{mL}$ and diluted in water to prepare working standard solutions. A working internal standard (deuterated MEHP $\left(\left[{ }^{2} \mathrm{H}_{4}\right] \mathrm{MEHP}\right)$, $\mathrm{C} / \mathrm{D} / \mathrm{N}$ Isotopes Inc., Pointe-Claire, Canada) solution was prepared similarly at $1 \mu \mathrm{g} / \mathrm{mL}$.

Plasma calibration standards were prepared at seven concentrations ( 25 to $5,000 \mathrm{ng} / \mathrm{mL})$ by spiking $25 \mu \mathrm{L}$ of plasma with an appropriate concentration of working MEHP standards. Plasma, amniotic fluid, and fetal homogenate quality control (QC) standards were prepared similarly at 100 and 2,500 ng/mL. Fetal homogenates were prepared by homogenizing fetuses in deionized water ( $1 \mathrm{~g}$ fetus in $3 \mathrm{~mL}$ water, equivalent to $250 \mathrm{mg} / \mathrm{mL}$ homogenate). Matrix blanks, method blanks, and study samples were prepared similarly to the matrix standards above but using $25 \mu \mathrm{L}$ of water in place of the MEHP working standard solution. To all samples, $25 \mu \mathrm{L}$ of internal standard solution and $425 \mu \mathrm{L}$ acetonitrile were added, vortexed, and centrifuged at approximately $8,000 \mathrm{~g}$ for 10 minutes at $4^{\circ} \mathrm{C}$. The supernatant was transferred to clean vials for analysis. Study samples with responses greater than the highest calibration standard were diluted with corresponding extracted blank matrix to the validated range prior to analysis.

\section{E.3. Instrumentation and Quantitation}

All samples were analyzed by ultra-performance liquid chromatography (UPLC) tandem mass spectrometry (MS/MS) using Waters ACQUITY UPLC (Milford, MA) coupled to an Applied Biosystems 4000 QTRAP (Sciex, Framingham, MA) mass spectrometer. Chromatography was performed using Waters ACQUITY UPLC BEH C18 column $(2.1 \times 50 \mathrm{~mm}, 1.8 \mu \mathrm{m})$. Mobile phases A (water) and B (acetonitrile) were run with a linear gradient from $20 \% \mathrm{~B}$ to $95 \% \mathrm{~B}$ in 4.5 minutes at a flow rate of $0.5 \mathrm{~mL} /$ minute. The electrospray ion source was operated in negative ion mode with a voltage of $-4,500$ volts and source temperature of $450^{\circ} \mathrm{C}$. Transitions monitored for MEHP and $\left[{ }^{1} \mathrm{H}_{4}\right]$ were $277.1 \rightarrow 133.8$ and $281.0 \rightarrow 137.8$, respectively.

Calibration curves relating the response ratio of analyte to internal standard and the concentration of MEHP in a matrix were constructed using a $1 / \mathrm{X}^{2}$ weighted linear regression. The concentrations of MEHP in the samples were calculated using response ratio, the regression 
equation, initial sample weight or volume, and dilution when applicable. The concentration was reported as $\mathrm{ng} / \mathrm{mL}$ for plasma and amniotic fluid. The fetal homogenate concentration estimated in $\mathrm{ng} / \mathrm{mL}$ of fetal homogenate was converted to $\mathrm{ng} / \mathrm{g}$ fetus by using a conversion factor of 4 (i.e., $4 \mathrm{~mL}$ homogenate $=1 \mathrm{~g}$ of fetus).

The performance of the calibration curve was evaluated before the analysis of each sample set. A successful calibration was indicated by the following: correlation coefficient $(r) \geq 0.99$; relative standard deviation (RSD) $\leq \pm 15 \%$ (except at the limit of quantitation [LOQ] where RSD is $\leq \pm 20 \%$ ); relative error (RE) $\leq \pm 15 \%$ (except at the LOQ where $\mathrm{RE}$ is $\leq \pm 20 \%$ ). Data from study samples were considered valid if they were bracketed by valid QC sets. In general, each sample set, method blanks, and controls were bracketed by two QC sets, which consisted of a calibration blank and two concentrations of calibration standards (QC low and QC high). A QC set passed when the measured concentration for QC standards was within 15\% of its nominal value. If the QC standard failed, it was necessary to reanalyze the bracketed samples. Correlation coefficient, $r$, for all calibration curves was $\geq 0.99$. All QC standards were within $15 \%$ of nominal concentrations for plasma, amniotic fluid, and fetal homogenates.

Low concentrations of MEHP were observed in plasma, amniotic fluid, and fetal homogenates used as blank matrices, some of which were attributed to background contributions from the reagents and vials used in the assay. The background contribution from the reagents/vials did not affect the quantitation of study samples because matrix calibration standards and study samples were prepared and quantified similarly. In general, the background concentrations estimated in amniotic fluid and fetal homogenates were slightly higher than those observed in plasma, although the reason is not clear.

Table E-1. Analytical Method Validation and Stability Data for Mono(2-ethylhexyl) Phthalate in Plasma, Amniotic Fluid, and Fetal Homogenate ${ }^{a}$

\begin{tabular}{|c|c|c|c|}
\hline Validation Parameter & Dam Plasma & Amniotic Fluid & Fetal Homogenate \\
\hline Matrix Concentration Range (ng/mL) & $25-5,000$ & - & - \\
\hline LOQ (ng/mL or $\mathrm{ng} / \mathrm{g}$ ) & 25.0 & 50.0 & 200 \\
\hline $\mathrm{LOD}^{\mathrm{b}}(\mu \mathrm{g} / \mathrm{g})$ & 5.2 & 12.0 & 10 \\
\hline Correlation Coefficient (r) & $\geq 0.998$ & - & - \\
\hline Selectivity $(\%)^{\mathrm{c}}$ & 40 & 53 & 54 \\
\hline Recovery $(\%)^{\mathrm{d}}$ & $95.0-102.0$ & - & - \\
\hline \multicolumn{4}{|l|}{ Precision and Accuracy ${ }^{\mathrm{e}, \mathrm{f}}$} \\
\hline Intra-day \% RSD & $\leq 4.1$ & $\leq 4.2$ & $\leq 7.1$ \\
\hline Intra-day \% RE & $\leq \pm 2.9$ & $\leq \pm 4.7$ & $\leq \pm 5.6$ \\
\hline Inter-day \% RSD & $\leq 5.2$ & - & - \\
\hline Inter-day \% RE & $\leq \pm 5.8$ & - & - \\
\hline \multicolumn{4}{|l|}{ Dilution Verification $(50,000 \mathrm{ng} / \mathrm{mL})$} \\
\hline$\%$ RSD & 0.8 & - & - \\
\hline$\% \mathrm{RE}$ & 1.7 & - & - \\
\hline Extracted Sample Storage Stability $(\% \text { of Day } 0)^{\mathrm{f}}$ & & & \\
\hline
\end{tabular}


Di(2-ethylhexyl) Phthalate, NTP TR 601

\begin{tabular}{lccc}
\hline \multicolumn{1}{c}{ Validation Parameter } & Dam Plasma & Amniotic Fluid & Fetal Homogenate \\
\hline Ambient & $102-105$ & - & - \\
Refrigerator & $97-107$ & - & - \\
Freeze-thaw (3 cycles) & $103-106$ & - & - \\
Matrix Storage Stability (\% of Day 0) & $80-113$ & $80-115$ & $84-113$ \\
\hline
\end{tabular}

$\mathrm{LOQ}=$ limit of quantitation; $\mathrm{LOD}=$ limit of detection; $\mathrm{RSD}=$ relative standard deviation; $\mathrm{RE}=$ relative error.

${ }^{a}$ Method was fully validated in Harlan Sprague Dawley rat plasma and assessed in amniotic fluid and fetal homogenate using quality control samples prepared in each matrix at three concentrations $(100,500$, and 2,500 ng/g).

${ }^{b}$ Estimated as the standard error of LOQ.

${ }^{\mathrm{c}}$ Estimated using six replicate blank matrix response relative to LOQ response.

${ }^{\mathrm{d} E s t i m a t e d ~ b y ~ c o m p a r i n g ~ r e s p o n s e ~ o f ~ m a t r i x ~ s t a n d a r d s ~ t o ~ s o l v e n t ~ s t a n d a r d s ~ o v e r ~ t h e ~ c o n c e n t r a t i o n ~ r a n g e ~} 25 \mathrm{to} 5,000 \mathrm{ng} / \mathrm{mL}$.

ePrecision was estimated as \% RSD. Accuracy was estimated as \% RE.

fDetermined for three replicate quality controls at three levels $(100,500$, and 2,500 ng/mL) for up to 3 days.

gDetermined for three replicate quality controls at two levels ( 100 and $3,750 \mathrm{ng} / \mathrm{mL})$ stored at $-20^{\circ} \mathrm{C}$ for up to 62 days. 


\section{Appendix F. Benchmark Dose Analysis}

\section{Table of Contents}

F.1. Methods

F-2

F.2. Results

\section{Tables}

Table F-1. Benchmark Dose Modeling Results for Hepatocellular Adenoma or

Carcinoma (Combined) in Male Rats in the Two-year Feed Studies of

Di(2-ethylhexyl) Phthalate

Table F-2. Benchmark Dose Modeling Results for Hepatocellular Adenoma or

Carcinoma (Combined) in Female Rats in the Two-year Feed Studies of

Di(2-ethylhexyl) Phthalate

Table F-3. Benchmark Dose Modeling Results for Pancreatic Acinar Adenoma or

Carcinoma (Combined) in Male Rats in the Two-year Feed Studies of

Di(2-ethylhexyl) Phthalate

F-6

Table F-4. Benchmark Dose Modeling Results for Uterine (Including Cervix)

Adenocarcinoma, Adenoma, Squamous Cell Carcinoma, or Squamous Cell

Papilloma (Combined) in Female Rats in the Two-year Feed Studies of

Di(2-ethylhexyl) Phthalate

\section{Figures}

Figure F-1. Benchmark Dose Modeling Results for Hepatocellular Adenoma or Carcinoma (Combined) in Male Rats in the Two-year Feed Studies of

Di(2-ethylhexyl) Phthalate. F-8

Figure F-2. Benchmark Dose Modeling Results for Hepatocellular Adenoma or

Carcinoma (Combined) in Female Rats in the Two-year Feed Studies of

Di(2-ethylhexyl) Phthalate.

Figure F-3. Benchmark Dose Modeling Results for Pancreatic Adenoma or Carcinoma

(Combined) in Male Rats in the Two-year Feed Studies of Di(2-ethylhexyl)

Phthalate

Figure F-4. Benchmark Dose Modeling Results for Uterine (Including Cervix)

Adenocarcinoma, Adenoma, Squamous Cell Carcinoma, or Squamous Cell

Papilloma (Combined) in Female Rats in the Two-year Feed Studies of

Di(2-ethylhexyl) Phthalate. 


\section{F.1. Methods}

Benchmark doses (BMDs) were calculated using the EPA Benchmark Dose Software (BMDS), version 3.1.2. ${ }^{169}$ The dose variable for the models was the amount of di(2-ethylhexyl) phthalate (DEHP) consumed $/ \mathrm{kg}$ body weight/day ( $\mathrm{mg} / \mathrm{kg} /$ day). Numbers of animals per exposure group were poly-3-adjusted survival numbers. The response variable was the incidence of the endpoint being modeled.

All of the frequentist dichotomous models in the BMDS were used. The logistic, log-probit, and probit models were used with no parameter restrictions. Other models (dichotomous Hill, gamma, log-logistic, multistage, and Weibull) were used with default restrictions on the ranges of some of the parameters, as described in the BMDS User Guide. ${ }^{170}$

The benchmark response (BMR) used in the models was $0.1(10 \%)$ extra risk, with estimated background levels. The benchmark dose lower confidence limit (BMDL 10$)$ was calculated using a 95\% confidence interval. The decision logic used to recommend one model from the fitted models was the default logic. ${ }^{170}$

\section{F.2. Results}

\section{F.2.1. Hepatocellular Adenoma or Carcinoma (Combined; Male Rats)}

\section{F.2.1.1. Perinatal and Postweaning Study}

All models provided an adequate fit to the data as assessed by a chi-square goodness-of-fit test $(p \geq 0.1)$ and by visual inspection of the respective plots of observed versus predicted values from the various models (Table F-1; Figure F-1). The dichotomous Hill, logistic, and probit models provided similar fits to the data. The probit model was judged to provide the best model fit based on the lowest Akaike information criterion (AIC) value. The BMDS dichotomous results for the probit and other models are available as supplemental data (Appendix $H$ ).

\section{F.2.1.2. Postweaning-only Study}

All models except for the dichotomous Hill and multistage degree 1 models provided an adequate fit to the data as assessed by a chi-square goodness-of-fit test $(p \geq 0.1)$ and by visual inspection of the respective plots of observed versus predicted values from the various models (Table F-1; Figure F-1). The multistage degree 4 and multistage degree 3 models provided similar fits to the data. The multistage degree 4 model was judged to provide the best model fit based on the lowest AIC value. The BMDS dichotomous results for the multistage degree 4 and other models are available as supplemental data (Appendix $\mathrm{H}$ ).

\section{F.2.2. Hepatocellular Adenoma or Carcinoma (Combined; Female Rats)}

\section{F.2.2.1. Perinatal and Postweaning Study}

All models except for the logistic and probit models provided adequate fits of the data as assessed by a chi-square goodness-of-fit test ( $\mathrm{p} \geq 0.1)$ and by visual inspection of the respective plots of observed versus predicted values from the various models (Table F-2; Figure F-2). The log-logistic and log-probit models provided similar fits of the data. The log-logistic model was 
judged to provide the best model fit based on the lowest AIC value. The BMDS dichotomous results for the log-logistic and other models are available as supplemental data (Appendix H).

\section{F.2.2.2. Postweaning-only Study}

All models provided adequate fits of the data as assessed by a chi-square goodness-of-fit test $(p \geq 0.1)$ and by visual inspection of the respective plots of observed versus predicted values from the various models (Table F-2; Figure F-2). The multistage degree 4, multistage degree 2, logistic, and probit models provided similar fits of the data. The multistage degree 4 model was judged to provide the best model fit based on the lowest AIC value. The BMDS dichotomous results for the multistage degree 4 and other models are available as supplemental data (Appendix H).

\section{F.2.3. Pancreatic Acinar Adenoma or Carcinoma (Combined; Male Rats)}

\section{F.2.3.1. Perinatal and Postweaning Study}

All models had poor goodness of fit $(\mathrm{p}<0.1)$. In addition, all models other than dichotomous Hill, log-logistic, and log-probit had high residuals near the $\mathrm{BMD}_{10}$. Of the models without high residuals near the $\mathrm{BMD}_{10}$, the dichotomous Hill model had the best fit based on AIC and was the only model with chi-square p value $>0.0001$ (Table F-3; Figure F-3). The dichotomous Hill model provided the best model fit based on the highest chi-square $\mathrm{p}$ value and lowest AIC value. The BMDS dichotomous results for the dichotomous Hill model and other models are available as supplemental data (Appendix H).

\section{F.2.3.2. Postweaning-only Study}

Only dichotomous Hill, log-logistic, and log-probit models provided adequate fits of the data as assessed by a chi-square goodness-of-fit test $(p \geq 0.1)$ and visual inspection of the respective plots of observed versus predicted values from the various models (Table F-3; Figure F-3). The dichotomous Hill, log-logistic, and log-probit models provided similar fits of the data. The loglogistic model provided the best model fit based on the highest chi-square $\mathrm{p}$ value and lowest AIC value. The BMDS dichotomous results for the log-logistic model and other models are available as supplemental data (Appendix $\mathrm{H}$ ).

\section{F.2.4. Uterine (Including Cervix) Adenocarcinoma, Adenoma, Squamous Cell Carcinoma, or Squamous Cell Papilloma (Combined; Female Rats)}

\section{F.2.4.1. Perinatal and Postweaning Study}

All models provided adequate fits of the data as assessed by a chi-square goodness-of-fit test $(p \geq 0.1)$ and visual inspection of the respective plots of observed versus predicted values from the various models (Table F-4; Figure F-4). The multistage degree 1, logistic, and probit models provided similar fits of the data. The logistic model provided the best model fit based on the lowest AIC value. The BMDS dichotomous results for the logistic and other models are available as supplemental data (Appendix $\mathrm{H}$ ).

\section{F.2.4.2. Postweaning-only Study}

All models provided adequate fits of the data as assessed by a chi-square goodness-of-fit test $(p \geq 0.1)$ and visual inspection of the respective plots of observed versus predicted values from the various models (Table F-4; Figure F-4). The multistage degree 1, logistic, and probit models 
provided similar fits of the data. The probit model provided the best model fit based on the lowest AIC value. The BMDS dichotomous results for the probit and other models are available as supplemental data (Appendix H).

Table F-1. Benchmark Dose Modeling Results for Hepatocellular Adenoma or Carcinoma (Combined) in Male Rats in the Two-year Feed Studies of Di(2-ethylhexyl) Phthalate

\begin{tabular}{|c|c|c|c|c|c|}
\hline Model & $\begin{array}{l}\text { Chi-square } \\
\text { P Value }^{\mathrm{a}}\end{array}$ & AIC & $\underset{(\mathrm{mg} / \mathrm{kg} / \mathrm{day})}{\mathrm{BMD}_{10}}$ & $\begin{array}{c}\text { BMDL }_{10} \\
(\mathrm{mg} / \mathrm{kg} / \mathrm{day})\end{array}$ & $\begin{array}{c}\text { BMDS } \\
\text { Recommendation }^{\mathrm{b}}\end{array}$ \\
\hline \multicolumn{6}{|c|}{ Perinatal and Postweaning Study (Study 1) } \\
\hline Dichotomous Hill & 0.55 & 92.9 & 199.5 & 168.2 & Viable-alternate \\
\hline Gamma & 0.48 & 93.4 & 328.0 & 199.7 & Viable-alternate \\
\hline Log-logistic & 0.48 & 93.4 & 326.1 & 194.6 & Viable-alternate \\
\hline Multistage Degree 4 & 0.45 & 93.6 & 356.5 & 198.5 & Viable-alternate \\
\hline Multistage Degree 3 & 0.45 & 93.6 & 356.5 & 198.5 & Viable-alternate \\
\hline Multistage Degree 2 & 0.45 & 93.6 & 356.5 & 198.5 & Viable-alternate \\
\hline Multistage Degree 1 & 0.38 & 93.6 & 249.9 & 161.3 & Viable-alternate \\
\hline Weibull & 0.47 & 93.5 & 336.2 & 198.8 & Viable-alternate \\
\hline Logistic & 0.61 & 92.0 & 413.6 & 335.9 & Viable-alternate \\
\hline Log-probit & 0.52 & 93.2 & 302.0 & 188.6 & Viable-alternate \\
\hline Probit & 0.63 & 91.8 & 382.9 & 306.1 & Viable-recommended \\
\hline \multicolumn{6}{|c|}{ Postweaning-only Study (Study 2) } \\
\hline Dichotomous Hill & 0.04 & 91.6 & 223.1 & 177.2 & Questionable \\
\hline Gamma & 0.14 & 89.6 & 416.2 & 262.1 & Viable-alternate \\
\hline Log-logistic & 0.14 & 89.6 & 428.0 & 259.7 & Viable-alternate \\
\hline Multistage Degree 4 & 0.26 & 87.6 & 434.4 & 263.5 & Viable-recommended \\
\hline Multistage Degree 3 & 0.26 & 87.61 & 434.4 & 263.5 & Viable-alternate \\
\hline Multistage Degree 2 & 0.19 & 88.0 & 375.0 & 248.1 & Viable-alternate \\
\hline Multistage Degree 1 & 0.07 & 91.0 & 273.0 & 173.8 & Questionable \\
\hline Weibull & 0.14 & 89.6 & 436.3 & 263.0 & Viable-alternate \\
\hline Logistic & 0.18 & 87.9 & 421.5 & 349.6 & Viable-alternate \\
\hline Log-probit & 0.13 & 89.6 & 393.9 & 250.8 & Viable-alternate \\
\hline Probit & 0.15 & 88.1 & 395.2 & 321.4 & Viable-alternate \\
\hline
\end{tabular}


Table F-2. Benchmark Dose Modeling Results for Hepatocellular Adenoma or Carcinoma (Combined) in Female Rats in the Two-year Feed Studies of Di(2-ethylhexyl) Phthalate

\begin{tabular}{|c|c|c|c|c|c|}
\hline Model & $\begin{array}{l}\text { Chi-square } \\
\text { P Value }\end{array}$ & AIC & $\begin{array}{c}\text { BMD }_{10} \\
\text { (mg/kg/day) }\end{array}$ & $\begin{array}{c}\text { BMDL }_{10} \\
\text { (mg/kg/day) }\end{array}$ & $\begin{array}{c}\text { BMDS } \\
\text { Recommendation }^{\mathrm{b}}\end{array}$ \\
\hline \multicolumn{6}{|c|}{ Perinatal and Postweaning Study (Study 1) } \\
\hline Dichotomous Hill & 0.14 & 143.1 & 77.6 & 41.5 & Viable-alternate \\
\hline Gamma & 0.14 & 142.7 & 153.7 & 105.9 & Viable-alternate \\
\hline Log-logistic & 0.24 & 141.5 & 122.9 & 79.7 & Viable-recommended \\
\hline Multistage Degree 4 & 0.14 & 142.7 & 153.7 & 105.9 & Viable-alternate \\
\hline Multistage Degree 3 & 0.14 & 142.7 & 153.7 & 105.9 & Viable-alternate \\
\hline Multistage Degree 2 & 0.14 & 142.7 & 153.7 & 105.9 & Viable-alternate \\
\hline Multistage Degree 1 & 0.14 & 142.7 & 153.7 & 105.9 & Viable-alternate \\
\hline Weibull & 0.14 & 142.7 & 153.7 & 105.9 & Viable-alternate \\
\hline Logistic & 0.02 & 148.2 & 348.5 & 274.2 & Questionable \\
\hline Log-probit & 0.27 & 142.1 & 92.0 & 41.5 & Viable-alternate \\
\hline Probit & 0.03 & 147.6 & 321.7 & 251.3 & Questionable \\
\hline \multicolumn{6}{|c|}{ Postweaning-only Study (Study 2) } \\
\hline Dichotomous Hill & 0.34 & 79.9 & 277.3 & 180.7 & Viable-alternate \\
\hline Gamma & 0.36 & 79.9 & 275.7 & 183.2 & Viable-alternate \\
\hline Log-logistic & 0.34 & 79.9 & 277.3 & 180.7 & Viable-alternate \\
\hline Multistage Degree 4 & 0.81 & 77.0 & 383.6 & 208.0 & Viable-recommended \\
\hline Multistage Degree 3 & 0.58 & 79.1 & 348.2 & 205.4 & Viable-alternate \\
\hline Multistage Degree 2 & 0.69 & 77.5 & 302.0 & 197.2 & Viable-alternate \\
\hline Multistage Degree 1 & 0.49 & 78.6 & 217.2 & 147.6 & Viable-alternate \\
\hline Weibull & 0.38 & 79.8 & 286.6 & 186.7 & Viable-alternate \\
\hline Logistic & 0.69 & 77.9 & 428.7 & 354.6 & Viable-alternate \\
\hline Log-probit & 0.36 & 80.2 & 338.5 & 168.0 & Viable-alternate \\
\hline Probit & 0.69 & 77.7 & 392.9 & 321.3 & Viable-alternate \\
\hline
\end{tabular}


Table F-3. Benchmark Dose Modeling Results for Pancreatic Acinar Adenoma or Carcinoma (Combined) in Male Rats in the Two-year Feed Studies of Di(2-ethylhexyl) Phthalate

\begin{tabular}{|c|c|c|c|c|c|}
\hline Model & $\begin{array}{l}\text { Chi-square } \\
\text { P Value }\end{array}$ & AIC & $\begin{array}{c}\text { BMD }_{10} \\
\text { (mg/kg/day) }\end{array}$ & $\begin{array}{c}\text { BMDL }_{10} \\
\text { (mg/kg/day) }\end{array}$ & $\begin{array}{c}\text { BMDS } \\
\text { Recommendation }^{\mathrm{b}}\end{array}$ \\
\hline \multicolumn{6}{|c|}{ Perinatal and Postweaning Study (Study 1) } \\
\hline Dichotomous Hill & 0.08 & 227.8 & 85.9 & 56.8 & Questionable \\
\hline Gamma & $<0.0001$ & 257.0 & 52.9 & 37.9 & Questionable \\
\hline Log-logistic & $<0.0001$ & 252.2 & 28.2 & 17.7 & Questionable \\
\hline Multistage Degree 4 & $<0.0001$ & 257.0 & 52.9 & 37.9 & Questionable \\
\hline Multistage Degree 3 & $<0.0001$ & 257.0 & 52.9 & 37.9 & Questionable \\
\hline Multistage Degree 2 & $<0.0001$ & 257.0 & 52.9 & 37.9 & Questionable \\
\hline Multistage Degree 1 & $<0.0001$ & 257.0 & 52.9 & 37.9 & Questionable \\
\hline Weibull & $<0.0001$ & 257.0 & 52.9 & 37.9 & Questionable \\
\hline Logistic & $<0.0001$ & 264.6 & 110.6 & 84.1 & Questionable \\
\hline Log-probit & $<0.0001$ & 251.5 & 33.1 & 13.5 & Questionable \\
\hline Probit & $<0.0001$ & 264.6 & 110.9 & 86.3 & Questionable \\
\hline \multicolumn{6}{|c|}{ Postweaning-only Study (Study 2) } \\
\hline Dichotomous Hill & 0.11 & 205.1 & 70.2 & 20.4 & Viable-alternate \\
\hline Gamma & 0.07 & 205.3 & 44.7 & 35.0 & Questionable \\
\hline Log-logistic & 0.13 & 204.6 & 31.0 & 20.2 & Viable-recommended \\
\hline Multistage Degree 4 & 0.07 & 205.3 & 44.7 & 35.0 & Questionable \\
\hline Multistage Degree 3 & 0.07 & 205.3 & 44.7 & 35.0 & Questionable \\
\hline Multistage Degree 2 & 0.07 & 205.3 & 44.7 & 35.0 & Questionable \\
\hline Multistage Degree 1 & 0.07 & 205.3 & 44.7 & 35.0 & Questionable \\
\hline Weibull & 0.07 & 205.3 & 44.7 & 35.0 & Questionable \\
\hline Logistic & 0.00 & 218.6 & 122.5 & 100.6 & Questionable \\
\hline Log-probit & 0.11 & 204.9 & 32.6 & 15.3 & Viable-alternate \\
\hline Probit & 0.00 & 217.6 & 115.8 & 96.9 & Questionable \\
\hline
\end{tabular}


Table F-4. Benchmark Dose Modeling Results for Uterine (Including Cervix) Adenocarcinoma, Adenoma, Squamous Cell Carcinoma, or Squamous Cell Papilloma (Combined) in Female Rats in the Two-year Feed Studies of Di(2-ethylhexyl) Phthalate

\begin{tabular}{|c|c|c|c|c|c|}
\hline Model & $\begin{array}{l}\text { Chi-square } \\
\text { P Value }\end{array}$ & AIC & $\begin{array}{c}\text { BMD }_{10} \\
\text { (mg/kg/day) }\end{array}$ & $\begin{array}{c}\text { BMDL }_{10} \\
\text { (mg/kg/day) }\end{array}$ & $\begin{array}{c}\text { BMDS } \\
\text { Recommendation }^{\text {b }}\end{array}$ \\
\hline \multicolumn{6}{|c|}{ Perinatal and Postweaning Study (Study 1) } \\
\hline Dichotomous Hill & 0.21 & 107.8 & 224.7 & 169.7 & Viable-alternate \\
\hline Gamma & 0.42 & 106.0 & 560.0 & 289.2 & Viable-alternate \\
\hline Log-logistic & 0.42 & 106.0 & 558.7 & 276.0 & Viable-alternate \\
\hline Multistage Degree 4 & 0.41 & 106.0 & 594.6 & 286.7 & Viable-alternate \\
\hline Multistage Degree 3 & 0.41 & 106.0 & 594.6 & 286.7 & Viable-alternate \\
\hline Multistage Degree 2 & 0.41 & 106.0 & 594.6 & 286.7 & Viable-alternate \\
\hline Multistage Degree 1 & 0.52 & 104.4 & 520.3 & 275.4 & Viable-alternate \\
\hline Weibull & 0.42 & 106.0 & 566.6 & 288.7 & Viable-alternate \\
\hline Logistic & 0.60 & 104.1 & 594.2 & 432.2 & Viable-recommended \\
\hline Log-probit & 0.43 & 105.9 & 524.7 & 254.6 & Viable-alternate \\
\hline Probit & 0.60 & 104.10 & 578.3 & 405.4 & Viable-alternate \\
\hline \multicolumn{6}{|c|}{ Postweaning-only Study (Study 2) } \\
\hline Dichotomous Hill & 0.33 & 147.6 & 180.6 & 116.6 & Viable-alternate \\
\hline Gamma & 0.25 & 148.4 & 273.3 & 144.1 & Viable-alternate \\
\hline Log-logistic & 0.25 & 148.4 & 267.5 & 127.5 & Viable-alternate \\
\hline Multistage Degree 4 & 0.25 & 148.5 & 272.6 & 143.1 & Viable-alternate \\
\hline Multistage Degree 3 & 0.25 & 148.5 & 272.9 & 143.1 & Viable-alternate \\
\hline Multistage Degree 2 & 0.25 & 148.5 & 272.6 & 143.1 & Viable-alternate \\
\hline Multistage Degree 1 & 0.41 & 146.6 & 224.4 & 141.7 & Viable-alternate \\
\hline Weibull & 0.25 & 148.4 & 272.6 & 143.9 & Viable-alternate \\
\hline Logistic & 0.39 & 146.7 & 344.0 & 268.9 & Viable-alternate \\
\hline Log-probit & 0.26 & 148.2 & 260.3 & 96.7 & Viable-alternate \\
\hline Probit & 0.41 & 146.6 & 324.1 & 249.0 & Viable-recommended \\
\hline
\end{tabular}


A.

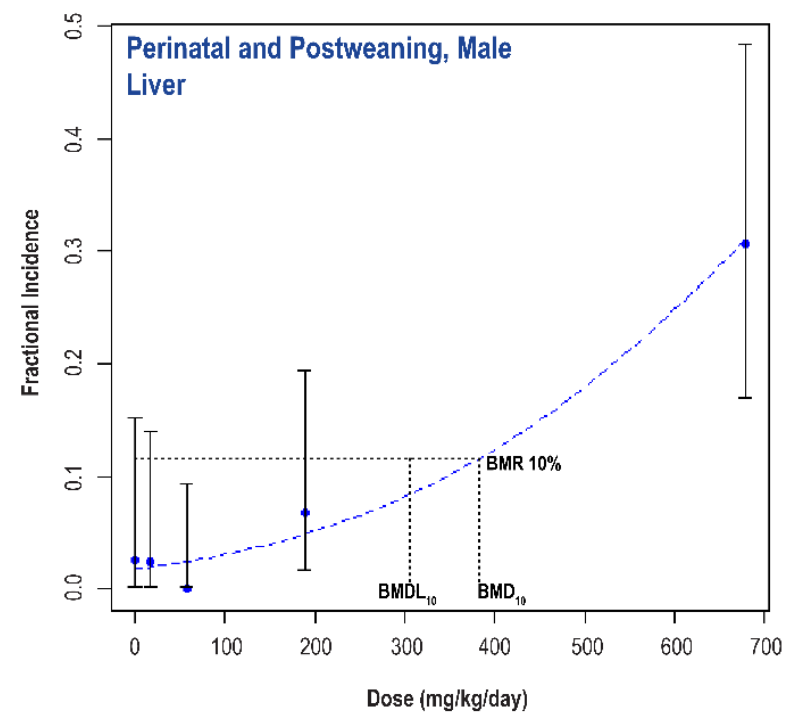

B.

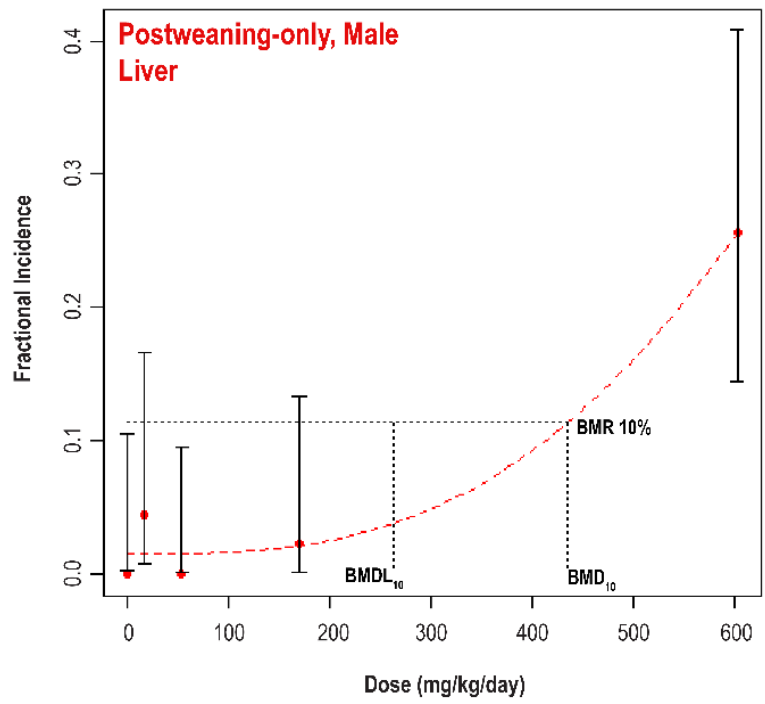

Figure F-1. Benchmark Dose Modeling Results for Hepatocellular Adenoma or Carcinoma (Combined) in Male Rats in the Two-year Feed Studies of Di(2-ethylhexyl) Phthalate

$\mathrm{BMR}=$ benchmark response; $\mathrm{BMD}_{10}=$ benchmark dose corresponding to a $10 \%$ extra risk; $\mathrm{BMDL}_{10}=95 \%$ lower bound on the benchmark dose corresponding to a $10 \%$ extra risk.

A.

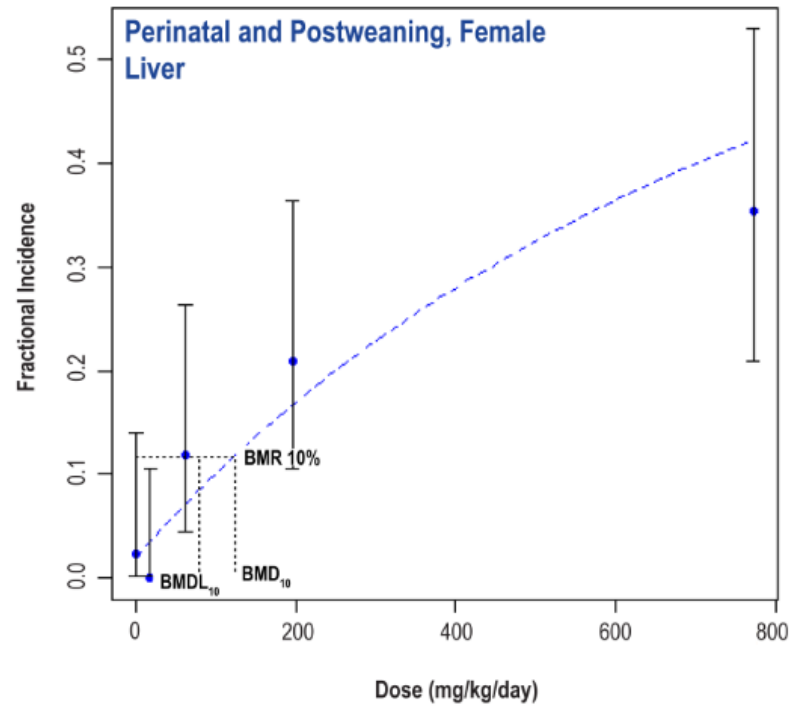

B.

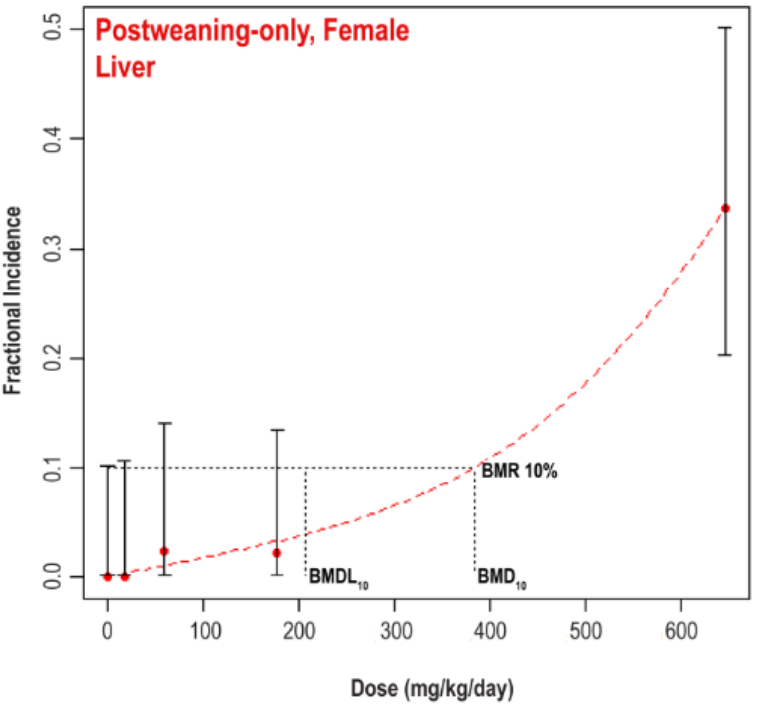

Figure F-2. Benchmark Dose Modeling Results for Hepatocellular Adenoma or Carcinoma (Combined) in Female Rats in the Two-year Feed Studies of Di(2-ethylhexyl) Phthalate

$\mathrm{BMR}=$ benchmark response; $\mathrm{BMD}_{10}=$ benchmark dose corresponding to a $10 \%$ extra risk; $\mathrm{BMDL}_{10}=95 \%$ lower bound on the benchmark dose corresponding to a $10 \%$ extra risk. 

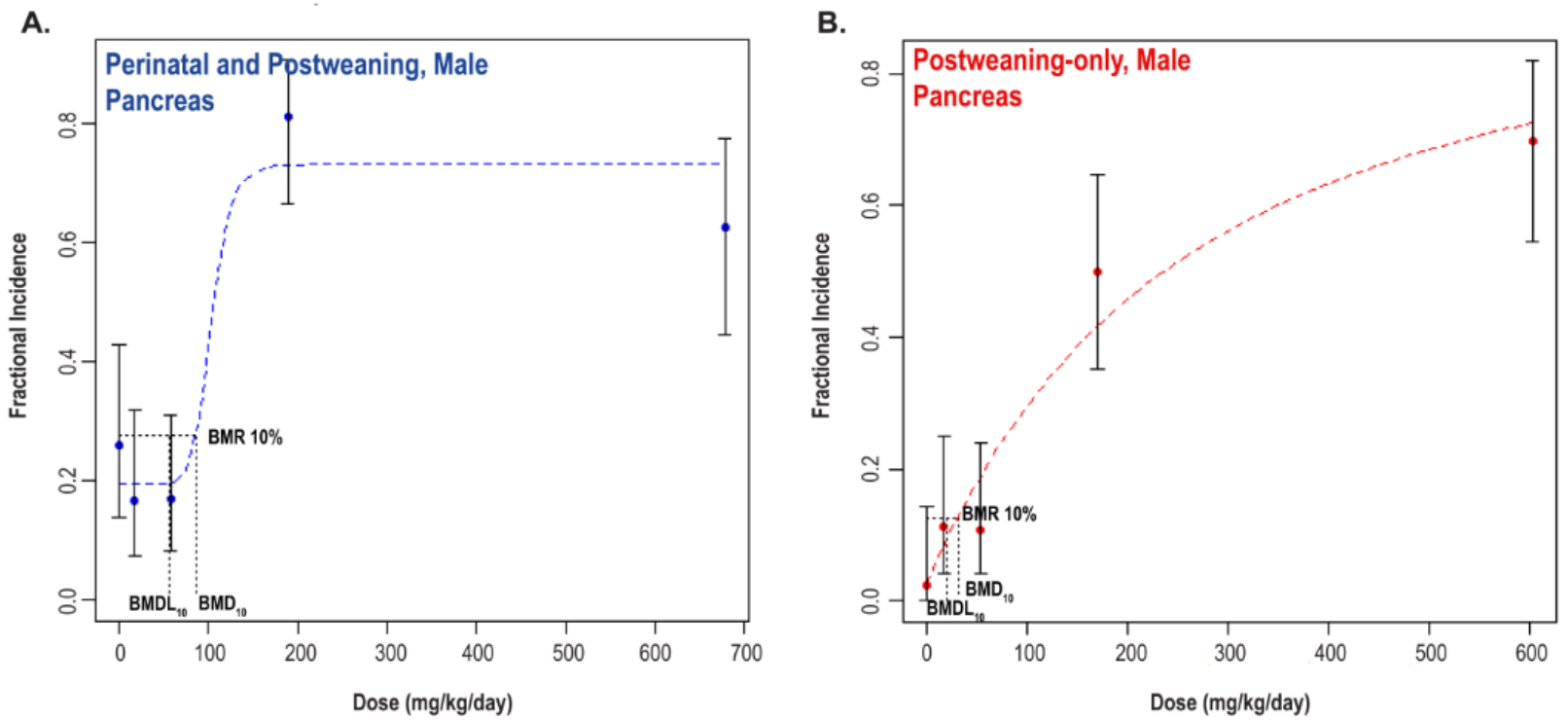

Figure F-3. Benchmark Dose Modeling Results for Pancreatic Adenoma or Carcinoma (Combined) in Male Rats in the Two-year Feed Studies of Di(2-ethylhexyl) Phthalate

$\mathrm{BMR}=$ benchmark response $\mathrm{BMD}_{10}=$ benchmark dose corresponding to a $10 \%$ extra risk; $\mathrm{BMDL}_{10}=95 \%$ lower bound on the benchmark dose corresponding to a $10 \%$ extra risk.

A.

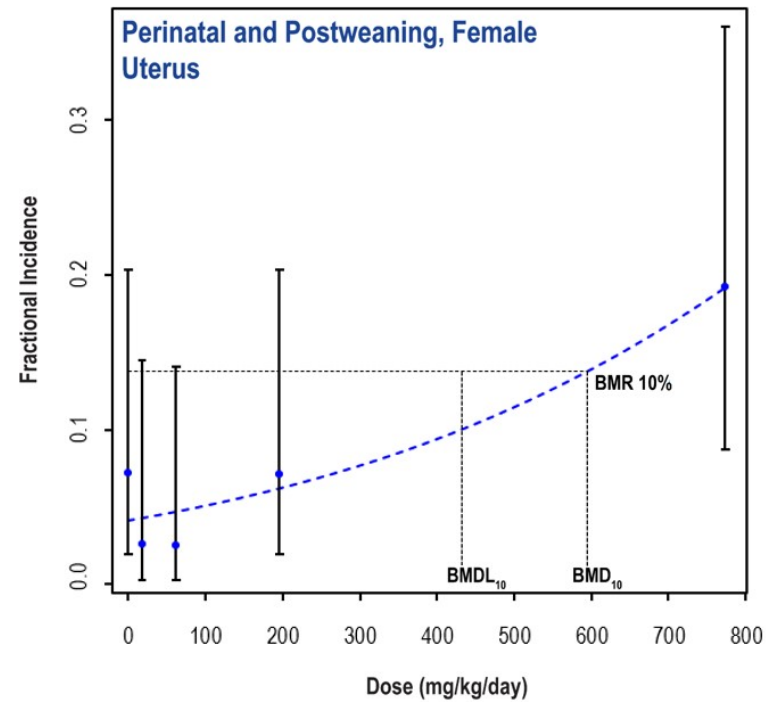

B.

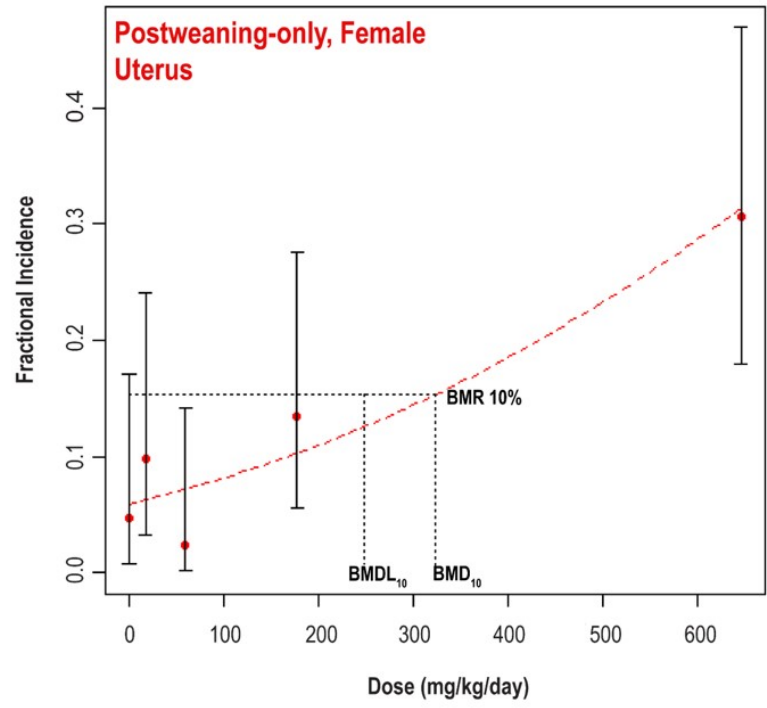

Figure F-4. Benchmark Dose Modeling Results for Uterine (Including Cervix) Adenocarcinoma, Adenoma, Squamous Cell Carcinoma, or Squamous Cell Papilloma (Combined) in Female Rats in the Two-year Feed Studies of Di(2-ethylhexyl) Phthalate

$\mathrm{BMR}=$ benchmark response; $\mathrm{BMD}_{10}=$ benchmark dose corresponding to a $10 \%$ extra risk; $\mathrm{BMDL}_{10}=95 \%$ lower bound on the benchmark dose corresponding to a $10 \%$ extra risk. 


\section{Appendix G. Peer-review Report}

\section{Table of Contents}

G.1. Attendees.

G-2

G.2. Peer Review of the Draft NTP Technical Reports on the Toxicology and

Carcinogenesis Studies of Sodium Tungstate Dihydrate, Di- $n$-butyl Phthalate, and

Di(2-ethylhexyl) Phthalate. 
The National Toxicology Program (NTP) virtually convened the NTP Technical Reports PeerReview Panel ("the Panel") on April 2, 2021, to peer review the Draft NTP Technical Reports on the Toxicology and Carcinogenesis Studies of Sodium Tungstate Dihydrate, Di-n-butyl Phthalate, and Di(2-ethylhexyl) Phthalate. Meeting information, including the draft reports, actions, and presentations is currently archived with NTP.

The panel peer reviewed the draft reports and provided its opinion on NTP's preliminary conclusions regarding the level of evidence of carcinogenic activity of sodium tungstate dihydrate, di-n-butyl phthalate, and di(2-ethylhexyl) phthalate. The panel's comments for the Draft NTP Technical Report on the Toxicology and Carcinogenesis Studies of Di(2-ethylhexyl) Phthalate (CASRN 117-81-7) Administered in Feed to Sprague Dawley (Hsd:Sprague Dawley ${ }^{\circledR}$ $\left.S D^{\circledR}\right)$ Rats begin at Section G.2.5. The panel's recommendations do not necessarily represent NTP's opinion.

\section{G.1. Attendees ${ }^{1}$}

\section{Peer-Review Panel}

Chair: Gabriele Ludewig, University of Iowa

Tracie E. Bunton, Eicarte, LLC

Michael R. Elwell, Apex Toxpath, LLC

Charles R. Mahrt, Retired, formerly with Flagship Biosciences

Daniel J. Spade, Brown University

John Pierce Wise, University of Louisville

\section{National Toxicology Program Board of Scientific Counselors Liaison}

Susan Felter, Procter \& Gamble

\section{National Institute of Environmental Health Sciences Staff}

Mamta Behl

Chad Blystone

Mark Cesta

Sheba Churchill

Helen Cunny

Susan Elmore

Dori Germolec

Michelle Hooth

Madelyn (Mimi) Huang

Angela King-Herbert

Barry McIntyre

Georgia Roberts

Sheena Scruggs, Designated Federal Official

Kelly Shipkowski

Keith Shockley

Robert Sills

Stephanie Smith-Roe

Suramya Waidyanatha

${ }^{1}$ The meeting was held via webcast. Individuals who viewed the webcast are not listed except as noted. 
Nigel Walker

Mary Wolfe

Other Federal Agency Staff

Shirisha Chittiboyina, National Institute for Occupational Safety and Health

Gonçalo Gamboa da Costa, U.S. Food and Drug Administration

Contract Support Staff

Amy Brix, EPL, Inc.

Canden Byrd, ICF

Lindsey Green, ICF

Shawn Harris, Social \& Scientific Systems, a DLH Company

Elizabeth Maull, Kelly Government Services

Megan Rooney, ICF

Alessandria Schumacher, ICF

Cynthia Willson, Integrated Laboratory Systems, LLC

\section{G.2. Peer Review of the Draft NTP Technical Reports on the Toxicology and Carcinogenesis Studies of Sodium Tungstate Dihydrate, Di-n-butyl Phthalate, and Di(2-ethylhexyl) Phthalate}

\section{G.2.1. Introductions and Welcome}

The National Toxicology Program (NTP) convened a peer-review panel for the Draft NTP Technical Reports on the Toxicology and Carcinogenesis Studies of Sodium Tungstate Dihydrate, Di-n-butyl Phthalate, and Di(2-ethylhexyl) Phthalate on April 2, 2021 via webcast.

- Dr. Gabriele Ludewig, panel chair, called the meeting to order at 10:00 a.m. and welcomed everyone to the meeting. She asked all attendees to introduce themselves, and reviewed the peer-review meeting format for the panel and audience.

- Dr. Mary Wolfe, Acting Deputy Director for Policy \& Communication, welcomed all participants to the meeting.

- Dr. Sheena Scruggs read the conflict-of-interest policy statement and briefed the attendees on meeting logistics.

- Dr. Susan Felter attended as the liaison to the NTP Board of Scientific Counselors.

- Dr. Shirisha Chittiboyina attended as the liaison for the National Institute for Occupational Safety and Health.

- Dr. Gonçalo Gamboa da Costa attended as the liaison for the U.S. Food and Drug Administration.

\section{G.2.2. Background and Charge to the Panel}

Dr. Chad Blystone briefly presented the NTP draft technical report objectives, including a review of the levels of evidence for the potential carcinogenic activity and factors considered for tested chemicals. He also described how NTP collects historical control data ${ }^{2}$ on neoplastic lesions and

\footnotetext{
${ }^{2}$ https://ntp.niehs.nih.gov/results/dbsearch/historical
} 
how these are utilized to provide context to report findings. Dr. Blystone provided the charge for the individual peer reviews:

- Review and evaluate the scientific and technical elements of the study and its presentation.

- Determine whether the study's experimental design, conduct, and findings support NTP's conclusions under the conditions of this study.

The peer-review meeting materials can be found on the NTP website.

\section{G.2.3. Toxicology and Carcinogenesis Studies of Sodium Tungstate Dihydrate}

\section{G.2.3.1. Presentation and Clarifying Questions}

Dr. Mamta Behl summarized the studies and conclusions reported in the Draft NTP Technical Report on the Toxicology and Carcinogenesis Studies of Sodium Tungstate Dihydrate (CASRN 10213-10-2) in Sprague Dawley (Hsd:Sprague Dawley $\left.{ }^{\circledR} S D^{\circledR}\right)$ Rats and B6C3F1/N Mice (Drinking Water Studies).

Tungsten occurs naturally in the environment and can enter waterways through the weathering of rocks and soil. It was nominated for study due to concerns about potential widespread human exposure via contaminated drinking water. Sodium tungstate dihydrate was selected because it is a naturally occurring, water-soluble form of tungsten. Drinking water was selected as the most likely route of exposure for the general population.

Dr. Behl first presented a summary of results from the perinatal and postweaning toxicity/carcinogenicity study in Hsd:Sprague Dawley ${ }^{\circledR} \mathrm{SD}^{\circledR}$ rats. NTP exposed time-mated female rats to $0,250,500$, or $1,000 \mathrm{mg} / \mathrm{L}$ sodium tungstate dihydrate in drinking water from gestational day (GD) 6 through postnatal day (PND) 21. NTP provided the $\mathrm{F}_{1}$ generation rats with the same respective sodium tungstate dihydrate concentrations as their dam for 2 years $\left(n=50 /\right.$ sex/concentration). In addition, $F_{1}$ generation rats were provided dosed drinking water or the vehicle control for 3, 6, 12, or 18 months for interim evaluations ( $n=40 /$ sex/concentration).

Dr. Behl then presented a summary of results from the chronic toxicity/carcinogenicity study in B6C3F1/N mice. NTP exposed mice to $0,500,1,000$, or $2,000 \mathrm{mg} / \mathrm{L}$ sodium tungstate dihydrate in drinking water for 2 years $(n=50 / \mathrm{sex} /$ concentration). An additional $40 \mathrm{mice} / \mathrm{sex} /$ concentration were included for interim evaluations at 3, 6, 12, and 18 months.

Under the conditions of these 2-year studies, NTP's draft conclusions were:

- No evidence of carcinogenic activity in male Hsd:Sprague Dawley ${ }^{\circledR} \mathrm{SD}^{\circledR}$ rats at 250 , 500 , and $1,000 \mathrm{mg} / \mathrm{L}$.

- Exposure to sodium tungstate dihydrate in drinking water caused increased incidences of nonneoplastic lesions in the kidney of male rats.

- Equivocal evidence of carcinogenic activity in female Hsd:Sprague Dawley ${ }^{\circledR} \mathrm{SD}^{\circledR}$ rats based on increased incidences of C-cell adenoma or carcinoma (combined) of the thyroid gland.

- Exposure to sodium tungstate dihydrate in drinking water caused increased incidences of nonneoplastic lesions in the kidney and uterus of female rats. 
- Equivocal evidence of carcinogenic activity in male $\mathrm{B} 6 \mathrm{C} 3 \mathrm{~F} 1 / \mathrm{N}$ mice based on the occurrences of renal tubule adenoma or carcinoma (combined) in exposed animals.

- Exposure to sodium tungstate dihydrate in drinking water caused increased incidences of nonneoplastic lesions in the kidney, testes, and bone marrow of male mice.

- No evidence of carcinogenic activity in female B6C3F1/N mice at 500, 1,000, and 2,000 mg/L sodium tungstate dihydrate.

- Exposure to sodium tungstate dihydrate in drinking water caused increased incidences of nonneoplastic lesions in the kidney and spleen of female mice.

There were no clarifying questions or comments about the presentation.

\section{G.2.3.2. Public Comments}

Dr. Ludewig acknowledged the receipt of one written public comment from Dr. Ranulfo Lemus on behalf of the International Tungsten Industry Association. Dr. Ludewig noted that the panel did not receive requests for oral public comments on the draft technical report.

\section{G.2.3.3. Peer-Review Comments and Panel Discussion}

\section{G.2.3.3.1. First Reviewer - Dr. Michael Elwell}

- Dr. Elwell indicated that the dose selection was appropriate, the studies were wellconducted, the results were discussed clearly, and the rationale was clearly presented for neoplastic findings of equivocal evidence and several nonneoplastic effects. The important sodium tungstate-related findings were well-described and represented by quality pathology images in the report.

- Dr. Elwell noted some inconsistencies across report sections on the relationship of atypical hyperplasia in the uterus of female rats to sodium tungstate dihydrate exposure. Text on page 104 of the draft report indicates that the relationship is unknown; however, text in the abstract, summary, and conclusions states that atypical hyperplasia in the uterus is a nonneoplastic effect caused by sodium tungstate dihydrate.

- Dr. Amy Brix stated that the sentence in the discussion about the unknown relationship to atypical hyperplasia was a typo and NTP may consider removing it. She stated that NTP believes that the effects were related to exposure and would consider make those edits.

- Dr. Elwell noted that on page 69 under "other tissues" for rats, several nonneoplastic findings were mentioned and considered to be of unknown biological significance. However, a significant decrease in fibroadenomas in the mammary gland was mentioned with no comment regarding biological significance or relationship to sodium tungstate dihydrate exposure. The fibroadenomas occurred in female rats with decreased body weights. He recommended that the report discuss the potential relevance of decreased body weight in relation to the tumors given that the effect of lower body weight on occurrence of this tumor has been noted in earlier NTP reports and in published studies.

- Dr. Brix said that information about the mammary gland would be considered by NTP along with citing Dr. Haseman's article that compares body weight 
changes to certain tumor incidences. For the nonneoplastic findings in other tissues, NTP can make it clear that they do not consider them toxicologically significant, treatment-related, or biologically significant.

- Dr. Elwell questioned the rationale for including two of the effects listed in the abstract, specifically increased spleen hematopoiesis and bone marrow hyperplasia. For both findings, the increased incidences occurred in the low and mid-exposure groups with no significant effect in the high-exposure group, and the average severity was similar across exposure groups. Given that the histopathology section states that the biological significance for the bone marrow and spleen is unknown, Dr. Elwell asked if there were other effects (e.g., inflammatory, hematologic, or hematopoietic) that supported listing these as effects in the abstract? As a point of reference, Dr. Elwell noted that NTP concluded that the kidney pigment findings, which were significantly higher in the high-exposure group, were of questionable toxicologic importance and therefore not brought into the abstract.

- Dr. Behl agreed with Dr. Elwell's comment on the spleen and explained that NTP noted an effect in bone marrow hyperplasia in males and hematopoietic cell proliferation in females at both low and mid doses. NTP is open to discussion about whether to bring these effects forward into the abstract. Dr. Behl asked if the panel recommended including spleen effects in the abstract.

- Dr. Elwell commented that the other effects in the abstract were doseor target organ-related; the spleen effects were weaker than what might be expected for a finding listed in the abstract.

- Dr. Brix noted that for the spleen and bone marrow, the incidences were two to three times higher in the low and mid-dose groups, but agreed that it is a weak connection and that NTP may be open to removing them from the abstract based on the panel discussion.

\section{G.2.3.3.2. Second Reviewer - Dr. John Pierce Wise}

- Dr. Wise concurred with Dr. Elwell. The report was well-written, and the study was well-designed.

- Given the pressure to evaluate environmentally relevant concentrations, Dr. Wise recommended that NTP provide language on dose selection rationale to help readers unfamiliar with the NTP approach.

- Dr. Behl noted that the comment on dose selection rationale was well-taken. She explained how NTP selected the concentrations and indicated that they have been criticized in the past for doses that failed to challenge the animals. The concentrations used in this study allowed NTP to state that tungstate at high levels does not result in overt toxicity. The effects observed in the kidney were common with this strain and species.

- Dr. Wise clarified that it was not that NTP should use different doses. Rather, he recommended that NTP specify that their intention is to determine whether a substance causes cancer, not to define whether a substance causes cancer at the most environmentally relevant dose. It 
would help to add that context for a reader who does not understand that approach.

- Dr. Behl said that NTP can add some clarifying language in the report.

- Dr. Wise recommended that NTP provide clarifying language to address the occurrence of pinworms in the rats for reader unfamiliar with rat use.

- Dr. Behl explained that the rats were positive for pinworms for the duration of the study, and they did not receive medication for elimination. At study termination, the incidences of pinworms were similar between the exposed groups. Based on histological sections, the pinworms were not associated with morphological changes in the large intestine and no inflammatory response was noted. NTP can clarify that point.

- Dr. Wise indicated it would be helpful to include that no medication was administered.

- Dr. Wise recommended that NTP add a second parameter in the table when presenting comet assay results, since it is standard to show two different measures such as tail length, olive moment, or tail moment.

- Dr. Stephanie Smith-Roe noted that NTP uses percent tail DNA, which is the OECD guideline for comet assay. Some comet assays report more than one measure, with those measures usually based on tail length. As there is variability in electrophoretic conditions that can influence tail length, NTP has found that percent tail DNA is a more reliable measure.

- Dr. Ludewig commented that she was unsure of how to interpret the significant comet effects even at the lowest concentration given the lack of pathology in the liver and other published positive comet assay results in the liver and other tissues.

- Dr. Smith-Roe replied that the results suggest that sodium tungstate may be capable of damaging DNA, but the liver was not a target organ for neoplastic effects.

\section{G.2.3.3.3. Third Reviewer - Dr. Charles Mahrt}

- Dr. Mahrt commented that the studies were well-designed, well-conducted, and the report was clear. However, he suggested NTP clarify whether the progression in rats from uterine atypical hyperplasia to adenocarcinomas (noted in Table 31) also included an increase in uterine adenomas.

- Dr. Brix agreed that it was unclear and indicated that NTP will consider adding language regarding the progression. From experience, adenomas are less common and NTP often sees a direct progression from atypical hyperplasia to adenocarcinomas.

\section{G.2.3.3.4. Panel Discussion}

Dr. Daniel Spade indicated he had no additional comments that were not already addressed by the other reviewers. 
Dr. Ludewig noted that the comet assay should be brought forward into the abstract since DNA damage was observed in the liver, despite no associated pathology.

Dr. Tracie Bunton asked about the justification for the large number of interim sacrifices and questioned whether NTP could have obtained information on tungsten accumulation using fewer sacrifices.

- Dr. Behl explained that the study was started several years ago when there were no data in the literature on the accumulation of tungsten in tissues following repeat dosing. Because the kidney is a target, NTP included multiple time points to determine if accumulation continued over the course of the study or if saturation eventually occurred. In addition, a question of sex differences required that NTP use males and females.

Dr. Bunton agreed with Dr. Mahrt's comment about Table 31 and thought the discussion was sufficient. She asked why Table 31 did not include incidence and statistics.

- Dr. Brix clarified that the adenocarcinomas and adenomas were included in Table 31 to show that the incidences were not significantly increased and that there was no progression from atypical hyperplasia. There were no statistics included because they were all negative.

Dr. Bunton noted that for female mice, there was a significant increase in the incidence of hepatocellular adenomas and carcinomas in the $500 \mathrm{mg} / \mathrm{L}$ group and that they were included in the "other tissues" groups. She asked why that was lumped into the "other tissues" category rather than brought into the tumor category.

- Dr. Brix stated that hepatocellular tumors are a common background lesion in this strain of mice, so it is not uncommon to have a dose group with significant differences due to biological variation. However, there was no dose response or reason to consider these treatments related.

Dr. Bunton asked for additional language or reorganization to be added in the discussion to explain how NTP came to an equivocal conclusion for female rats. NTP stated that the conclusion was based on increased incidences of C-cell adenoma and carcinoma (combined) of the thyroid gland, but the statement is incomplete as written because that same rationale could apply for a carcinogenic conclusion.

- Dr. Brix said that NTP could look at the discussion and clarify.

Dr. Ludewig asked if NTP had any information about adipose tissue or lipid content in the liver, given that absolute liver weight decreased, there were positive comet assay results in the males, and the text mentions that sodium tungstate dihydrate is an antidiabetic agent.

- Dr. Nigel Walker said that NTP does not have these data.

\section{G.2.3.4. Vote on NTP Conclusions}

\section{G.2.3.4.1. Male Hsd:Sprague Dawley ${ }^{\circledR} S D^{\circledR}$ rats}

Dr. Ludewig called for a motion from the panel to approve the conclusions as written. Dr. Wise so moved, and Dr. Mahrt seconded the motion. The panel voted unanimously ( 5 yes, 0 no, 0 abstentions) to approve the conclusions as written. 


\section{G.2.3.4.2. Female Hsd:Sprague Dawley ${ }^{\circledR} S D^{\circledR}$ rats}

Dr. Ludewig called for a motion from the panel to approve the conclusions as written. Dr. Wise so moved, and Dr. Elwell seconded the motion. The panel voted unanimously ( 5 yes, 0 no, 0 abstentions) to approve the conclusions as written.

\section{G.2.3.4.3. Male B6C3F1/N mice}

Dr. Ludewig called for a motion from the panel to approve the conclusions as written. Dr. Wise so moved, and Dr. Elwell seconded the motion. The panel voted unanimously ( 5 yes, 0 no, 0 abstentions) to approve the conclusions as written.

\section{G.2.3.4.4. Female B6C3F1/N mice}

Dr. Ludewig called for a motion from the panel to approve the conclusions as written. Dr. Wise so moved, and Dr. Mahrt seconded the motion. The panel voted unanimously ( 5 yes, 0 no, 0 abstentions) to approve the conclusions as written.

\section{G.2.4. Toxicology and Carcinogenesis Studies of Di-n-butyl Phthalate}

\section{G.2.4.1. Presentation and Clarifying Questions}

Dr. Madelyn (Mimi) Huang summarized the studies and conclusions reported in the Draft NTP Technical Report on the Toxicology and Carcinogenesis Studies of Di-n-butyl Phthalate (CASRN 84-74-2) Administered in Feed to Sprague Dawley (Hsd:Sprague Dawley ${ }^{\circledR} S D^{\circledR}$ ) Rats and B6C3F1/N Mice.

Di-n-butyl phthalate (DBP) is commonly used as a plasticizer and is found in a variety of consumer products, such as vinyl fabrics and flooring, personal care products, pharmaceuticals, and food packaging. Human exposure primarily occurs through ingestion of food packaged in materials containing DBP; some inhalation and dermal exposure occurs as well, but to a lesser extent. In the gut, DBP is rapidly metabolized to mono-n-butyl phthalate (MBP) and undergoes broad distribution throughout the body.

Dr. Huang presented a summary of results from the perinatal and postweaning toxicity and carcinogenicity study in Hsd:Sprague Dawley ${ }^{\circledR} \mathrm{SD}^{\circledR}$ rats. Time-mated female rats were fed diets containing $0,300,1,000,3,000$, or 10,000 ppm DBP from gestation day (GD) 6 through postnatal day (PND) 21. NTP provided F1 generation rats with the same respective DBP concentrations in feed as their dam for 2 years (generally two/sex/litter). In addition, select dams and their litters were removed on GD 18 and lactation day (LD) 4 to quantify the internal concentration of MBP.

Dr. Huang then presented a summary of results from the chronic toxicity and carcinogenicity study in B6C3F1/N mice. Mice were fed diets containing 0, 1,000, 3,000, or 10,000 ppm DBP for 2 years $(n=50 /$ sex/group).

Under the conditions of these 2-year studies, NTP's draft conclusions were:

- Equivocal evidence of carcinogenic activity in male Hsd:Sprague Dawley ${ }^{\circledR} \mathrm{SD}^{\circledR}$ rats based on marginal increases in the incidence of pancreatic acinus adenomas. 
- Exposure to DBP resulted in increased incidences of gross lesions of the male reproductive system and nonneoplastic lesions of the male reproductive system, liver, and pituitary gland pars distalis in male rats.

- No evidence of carcinogenic activity in female Hsd:Sprague Dawley ${ }^{\circledR} \mathrm{SD}^{\circledR}$ rats at $300,1,000,3,000$, or 10,000 ppm.

- Exposure to DBP resulted in increased incidences of nonneoplastic lesions of the liver in female rats.

- No evidence of carcinogenic activity in male B6C3F1/N mice at 1,000, 3,000, or $10,000 \mathrm{ppm}$.

- Exposure to DBP increased incidences of nonneoplastic lesions of the male reproductive system and liver in male mice.

- No evidence of carcinogenic activity in female B6C3F1/N mice at 1,000, 3,000, or $10,000 \mathrm{ppm}$.

- Exposure to DBP increased incidences of nonneoplastic lesions of the liver and kidney in female mice.

There were no clarifying questions or comments about the presentation.

\section{G.2.4.2. Public Comments}

Dr. Gabriele Ludewig acknowledged that there were no written public comments on the draft technical report. She also noted that the panel did not receive requests for oral public comments on the draft technical report.

\section{G.2.4.3. Peer-Review Comments and Panel Discussion}

\section{G.2.4.3.1. First Reviewer-Dr. Tracie Bunton}

- Dr. Bunton said the presentation of the rationale, methods, and results was clear and concise.

- She commented that she liked the introduction and found it helpful in getting up to speed on the properties, uses, and reduction in use of DBP. The rationale for the overall significance of the study is solid, especially given that perinatal exposure is a knowledge gap.

- She indicated that all the information about stability, homogeneity, dose selection, and number of animals per dose group is appropriate for the design of the study.

- Dr. Bunton suggested including the word "microscopic" or eliminate the word "gross" in the conclusion statement of the report as it currently specifies gross lesions in the male reproductive system but does not specify that the nonneoplastic lesions are not gross lesions.

○ Dr. Huang indicated that NTP is open to adding in the word "microscopic" to differentiate from gross lesions.

- Dr. Bunton noted that the findings to support the "equivocal" decision included an increased incidence in pancreatic acinar adenomas compared to controls, without a concurrent increase in hyperplasia, and a significant positive trend. 
- She stated the lesion in the liver was compatible with effects of other phthalates, namely increased cytoplasmic alteration. This was an important point that was noted in the pathology review and in the discussion.

- She also agreed that the findings fit into the "equivocal" category.

\section{G.2.4.3.2. Second Reviewer - Dr. Charles Mahrt}

- Dr. Mahrt agreed with Dr. Bunton's comments and noted that the report was welldesigned, conducted, and written.

- He appreciated the references to the literature to put the findings in perspective, especially the possibility of lesions related to peroxisome proliferation.

\section{G.2.4.3.3. Third Reviewer-Dr. Daniel Spade}

- Dr. Spade indicated that the study was well-conducted, and the report was clearly written.

- He noted that in the abstract, lines 39 to 40 indicate that there were fewer and less severe reproductive lesions in mice than in rats. He agreed with that conclusion, though suggested qualifying it by acknowledging the limits of cross-species comparison given that rats had perinatal exposure and mice did not.

- Dr. Huang agreed that it was important to qualify the rat versus mouse comparison and said NTP could clarify those statements.

- Dr. Nigel Walker explained that when NTP started adding the perinatal component to rat studies, people were trying to understand why the study design changed. With rat studies as perinatal and mouse studies as adults only, NTP has started to address that in the mid-2000s. In addition, it is difficult to do perinatal exposure on $\mathrm{F}_{1}$ generation $\mathrm{B} 6 \mathrm{C} 3 \mathrm{~F} 1 / \mathrm{N}$ mice.

- Dr. Spade built on Dr. Bunton's comment about the equivocal conclusion for pancreatic acinar adenomas and asked if there was a significant trend test but not pairwise test.

- Dr. Huang stated the affirmative.

- Dr. Spade appreciated the response and agreed with the conclusion.

- He also asked why images were not included in some cases.

- Dr. Huang clarified that NTP does not generally include images for common lesions or when the conclusion is equivocal.

- Dr. Spade appreciated the response and agreed with the approach.

\section{G.2.4.3.4. Panel Discussion}

Dr. Michael Elwell noted that the last line of the discussion mentions that 2,4dichlorophenoxyacetic acid and DBP did not produce hepatic lesions typical of peroxisome proliferators. DBP did result in hepatic lesions but did not produce hepatic neoplasms.

- Dr. Huang agreed that the last line should reference neoplasms instead of lesions, as Dr. Elwell suggested. 
Regarding voting, Dr. Elwell noted that the original draft report mentions hypertrophy in the pituitary and hyperplasia, but that it was not mentioned in the oral presentation. He asked if hypertrophy was included in the pituitary findings.

- Dr. Mark Cesta explained that NTP relooked at the data about the pituitary and hyperplasia. Hyperplasia often occurs with hypertrophy lesions, so they wanted to explain the observed hyperplasia. However, after a closer look, NTP could not make that conclusion and decided to remove it from the abstract.

Dr. Ludewig noted that page 78 of the draft report explains the concentrations found in the amniotic fluid. When humans and rats both take up $1 \mathrm{mg} / \mathrm{kg} / \mathrm{day}$, a historic report showed that there were $22 \mathrm{ng} / \mathrm{mL}$ of the metabolite in the amniotic fluid of humans. However, NTP only found $5 \mathrm{ng} / \mathrm{mL}$ in rats. Dr. Ludewig said she was initially surprised until she noticed that NTP did not measure the glucuronic acid conjugate. She wondered if that would also cross the placenta or if conjugation would protect the fetus. She suggested adding a statement that the gluconate conjugate was not measured.

- Dr. Huang agreed that there are differences in distribution and metabolism between rodents and humans. She was unsure if glucuronidation affects its ability to cross the placenta and said that was something NTP would look into.

\section{G.2.4.4. Vote on NTP Conclusions}

\section{G.2.4.4.1. Male Hsd:Sprague Dawley ${ }^{\circledR} S D^{\circledR}$ rats}

Dr. Ludewig called for a motion from the panel to approve the conclusions as written. Dr. Bunton so moved, and Dr. Mahrt seconded the motion. The panel voted unanimously (5 yes, 0 no, 0 abstentions) to approve the conclusions as written.

\section{G.2.4.4.2. Female Hsd:Sprague Dawley ${ }^{\circledR} S D^{\circledR}$ rats}

Dr. Ludewig called for a motion from the panel to approve the conclusions as written. Dr. John Pierce Wise so moved, and Dr. Elwell seconded the motion. The panel voted unanimously (5 yes, 0 no, 0 abstentions) to approve the conclusions as written.

\section{G.2.4.4.3. Male B6C3F1/N mice}

Dr. Ludewig called for a motion from the panel to approve the conclusions as written. Dr. Bunton so moved, and Dr. Wise seconded the motion. The panel voted unanimously ( 5 yes, 0 no, 0 abstentions) to approve the conclusions as written.

\section{G.2.4.4.4. Female B6C3F1/N mice}

Dr. Ludewig called for a motion from the panel to approve the conclusions as written. Dr. Wise so moved, and Dr. Bunton seconded the motion. The panel voted unanimously ( 5 yes, 0 no, 0 abstentions) to approve the conclusions as written.

\section{G.2.5. Toxicology and Carcinogenesis Studies of Di(2-ethylhexyl) Phthalate}

\section{G.2.5.1. Presentation and Clarifying Questions}

Dr. Chad Blystone summarized the studies and conclusions reported in the Draft NTP Technical Report on the Toxicology and Carcinogenesis Studies of Di(2-ethylhexyl) Phthalate (CASRN 117-81-7) Administered in Feed to Sprague Dawley (Hsd:Sprague Dawley $\left.{ }^{\circledR} S D^{\circledR}\right)$ Rats. 
Di(2-ethylhexyl) phthalate (DEHP) is a phthalate ester that was widely used in manufacturing of PVC polymers and corresponding products, such as cosmetics and toys. Over the years DEHP use has declined due to toxicity concerns, but chronic exposure throughout multiple life stages still occurs. The literature suggests that exposure to DEHP during an early life stage may result in chronic or carcinogenic health outcomes. However, previous DEHP chronic rodent studies did not include exposure during the gestational period up to weaning in rodents. To address this, NTP conducted two comparative DEHP carcinogenesis studies in rats to determine if including early life exposure would alter chronic toxicity or carcinogenicity outcomes.

Dr. Blystone presented a summary of results from the perinatal and postweaning toxicity/carcinogenicity study in Hsd:Sprague Dawley ${ }^{\circledR} \mathrm{SD}^{\circledR}$ rats. In the perinatal and postweaning study, time-mated female rats were fed diets containing $0,300,1,000,3,000$, or $10,000 \mathrm{ppm}$ DEHP from gestational day (GD) 6 through postnatal day (PND) 21 ( $\mathrm{n}=45 /$ group). Select dams were removed on GD 18 to quantify internal concentrations of a metabolite of DEHP, mono(2-ethylhexyl) phthalate, in plasma and tissue samples. NTP provided $\mathrm{F}_{1}$ generation rats with the same respective DEHP concentration in feed as their dam for 2 years ( $\mathrm{n}=2 / \mathrm{sex} /$ litter; $\mathrm{n}=50$ total $/$ sex/group).

Dr. Blystone then presented a summary of results from the postweaning toxicity/carcinogenicity study in Hsd:Sprague Dawley ${ }^{\circledR} \mathrm{SD}^{\circledR}$ rats. In the postweaning-only study, rats were fed diets containing $0,300,1,000,3,000$, or 10,000 ppm DEHP for 2 years ( $\mathrm{n}=50 / \mathrm{sex} /$ group).

Under the conditions of these 2-year studies, NTP's draft conclusions were:

- Perinatal and Postweaning Feed Study:

- Clear evidence of carcinogenic activity in male Hsd:Sprague Dawley ${ }^{\circledR} \mathrm{SD}^{\circledR}$ rats based on the increased incidences of hepatocellular adenoma or carcinoma (combined) and acinar adenoma or carcinoma (combined) neoplasms (predominantly adenomas) of the pancreas.

- Exposure to DEHP resulted in increased incidences of nonneoplastic lesions in the liver, heart, pituitary gland, testis, and epididymis and increased incidences of gross lesions of the reproductive tract, bone marrow, and kidney in male rats.

- Clear evidence of carcinogenic activity in female Hsd:Sprague Dawley ${ }^{\circledR} \mathrm{SD}^{\circledR}$ rats based on the increased incidence of hepatocellular adenoma or carcinoma (combined).

- The occurrence of pancreatic acinar adenoma or carcinoma (combined) was considered to be related to exposure. (Some evidence)

- The occurrence of uterine (including cervix) adenoma, adenocarcinoma, squamous cell carcinoma, or squamous cell papilloma (combined) in female rats may have been related to exposure. (Equivocal evidence)

- Exposure to DEHP resulted in increased incidences of nonneoplastic lesions in the liver and increased incidences of gross lesions of the kidney in female rats.

- Postweaning-only Feed Study 
- Clear evidence of carcinogenic activity in male Hsd:Sprague Dawley ${ }^{\circledR} \mathrm{SD}^{\circledR}$ rats based on the increased incidences of hepatocellular adenoma or carcinoma (combined) and increased incidences of acinar adenoma or carcinoma (combined) neoplasms (predominantly adenomas) of the pancreas.

- The occurrence of testicular interstitial cell adenoma in male rats may have been related to exposure. (Equivocal evidence)

- Exposure to DEHP resulted in increased incidences of nonneoplastic lesions in the liver, pancreas, bone marrow, heart, pituitary gland, testis, and epididymis.

○ Clear evidence of carcinogenic activity in female Hsd:Sprague Dawley ${ }^{\circledR} \mathrm{SD}^{\circledR}$ rats based on the incidences of hepatocellular adenoma or carcinoma (combined) and uterine (including cervix) adenoma, adenocarcinoma, squamous cell carcinoma, or squamous cell papilloma (combined).

- The occurrence of pancreatic acinar adenoma or carcinoma (combined) in female rats was considered to be related to exposure. (Some evidence)

- Exposure to DEHP resulted in increased incidences of nonneoplastic lesions in the liver, pancreas, bone marrow, and uterus in female rats.

- Comparative Carcinogenic Benchmark Dose Analyses

- No consistent pattern indicating that perinatal and postweaning exposure was more sensitive compared to postweaning-only exposure and modeled responses were within threefold of each other.

- However, there was a stronger carcinogenic response in the reproductive organs (uterus and testis) in the postweaning-only exposure study compared to the perinatal and postweaning exposure study.

Dr. Gabriele Ludewig asked a clarifying question about a shift in male to female fetus ratios that was not mentioned during Dr. Blystone's talk. Dr. Blystone responded that female fetuses were lost at the highest concentration. The reduction in litter size was due to this but was inconsistent and not considered related to DEHP exposure.

\section{G.2.5.2. Public Comments}

Dr. Ludewig acknowledged that there were no written public comments submitted on the draft technical report. She noted that the panel also did not receive requests for oral public comments on the draft technical report.

\section{G.2.5.3. Peer-Review Comments and Panel Discussion}

\section{G.2.5.3.1. First Reviewer - Dr. John Pierce Wise}

- Dr. Wise indicated that the study was well-designed, well-conducted, and clear in its data presentation.

- He suggested that NTP clarify that pinworm infections were not treated with medication.

○ Dr. Blystone said that NTP can add this clarification. 
- Dr. Wise also reiterated his comment from sodium tungstate dihydrate that NTP should explain that the choice of dose was deliberate and not intended to test the most environmentally relevant level of exposure, but rather to test whether the substance is carcinogenic.

- Dr. Blystone agreed that the point of these studies is hazard characterization and noted that NTP can clarify that in the report and include it in the lay summary.

- Dr. Wise suggested that NTP could add this in a dose selection rationale section.

○ Dr. Blystone responded that the reports have a section covering the technical aspects of the exposure concentration selection and that a sentence can be added to highlight rationale for exposure concentrations.

\section{G.2.5.3.2. Second Reviewer-Dr. Daniel Spade}

- Dr. Spade stated that the report was thorough and clear; it makes a massive amount of work easily understandable.

- He asked for clarification in how the reduction in litter size was presented, and asked whether there was an effort to address post-implantation loss as part of the reduced litter size. Reduced litter size could not be due to exposure related pre-implantation loss since the dosing window did not begin before implantation. However, Dr. Spade argued that with exposures beginning on GD 6, this overlaps with organogenesis and poses a risk for post-implantation loss which has been reported in the phthalate literature.

- Dr. Blystone said that NTP only evaluated post-implantation loss in females which did not deliver, so there are no more data available on postimplantation loss and litter size.

- Dr. Spade asked why pup survival data for PND 1-4 and PND 5-21 on page 29 of the draft report were analyzed separately. Dr. Spade thought that there could have been a trend in mortality if PND 1-21 were analyzed together.

- Dr. Blystone explained that NTP typically standardizes the litter size on PND 4, so the analysis looks at early (PND 1-4) and later (PND 5-21) mortality. Dr. Blystone acknowledged that based on Dr. Spade's written comment NTP reanalyzed the data after combining the two periods and there was still no significant trend or pairwise comparison.

- Dr. Spade also noted that it was unclear why some lesions of unknown biological significance on page 61 of the draft report were classified as such. Of note were the adrenal gland lesions, because of the known antiandrogenic effect of DEHP. Also, he questioned the ovarian atrophy as classified as unknown biological significance, because published data from academic studies indicate that phthalates change the rates of follicle maturation which could be related.

- Dr. Blystone indicated that NTP can clarify the language and focus more on toxicological significance. 
- Dr. Spade commented that 24 months is reproductively aged, so for certain findings such as seminiferous epithelium degeneration, the control levels are very high which makes it less likely that there will be a significant pairwise test. This limits the ability to know with certainty what the dose response would look like for an endpoint such as epithelial degeneration. If 4-month-old males were tested, you might see a significant response at lower levels. The study supports the conclusions within the constraints of the study, but this is a limitation.

- Dr. Blystone agreed that at this age, the model is not very sensitive. NTP can add a statement about that to the report.

- Dr. Spade noted that for gestational transfer, as discussed on page 33 of the draft report, one limitation is that DEHP has many secondary metabolites. He noted that without measuring these secondary metabolites it is difficult to determine the total transfer.

- Dr. Blystone agreed and said that NTP can clarify that metabolites can be transferred at different rates.

Dr. Ludewig stated that it was interesting that mono(2-ethylhexyl) phthalate was found in the amniotic fluid and the fetus of the control animals, but nothing in the serum of dams.

- Dr. Blystone agreed that this was unusual and noted that sometimes sample preparations can lead to irregularities.

- Dr. Suramya Waidyanatha said NTP concluded it was probably due to contamination of samples during collection or preparation and that this is likely due to the small volume of these samples.

\section{G.2.5.3.3. Third Reviewer - Dr. Michael Elwell}

- Dr. Elwell agreed with previous reviewers that the results are clearly presented and discussed.

- He noted that at the highest dose, body weights were reduced by approximately $30 \%$ in the perinatal postweaning and approximately $20 \%$ in the postweaning study. Although this did not affect survival, the decreased incidence of several neoplastic and nonneoplastic findings (especially for the high dose group of each study) might be attributable to significantly lower body weights. In the report (pages 61 and 77) these are indicated to be of unknown biological significance. If these findings are due to lower body weight, they should be addressed as such rather than reported as unknown significance. For example, neoplasms including the c-cell, pituitary, and mammary gland tumors can be affected by body weight. In females, a single pituitary gland neoplasm reported at the high dose is unexpected and notable in comparison to the control group where 16 animals were reported to have this tumor. The decreased incidence of the nonneoplastic lesions of testis polyarteritis and parathyroid hyperplasia could also be related to the lowered severity of chronic progressive nephropathy (CPN). In both studies, CPN was decreased in severity at the high dose in both studies and indicated as the cause of death in 0 and 1 animal while 16-18 animals in the control groups list CPN as the cause of death. This report cites other DEHP studies that attribute an increase in CPN to DEHP while this report suggests the opposite effect on CPN. This may be because the highest doses in this study were 
lower, the decreased body weight, or that the NTP 2000 diet was not used in earlier studies.

- Dr. Blystone indicated that NTP can clarify biological significance versus toxicological significance. Dr. Blystone agreed that some of the effects could be related to decreased body weight and stated that NTP can clarify.

- Dr. Elwell asked if page 87 of the draft report should state that it is unclear if any difference corresponded to developmental mechanisms when comparing the two studies, or if it is a typo. It appeared the kidney was affected by a developmental mechanism and DEHP was presumed to interfere with proper development.

- Dr. Blystone stated that NTP can fix the typo.

- Dr. Elwell noted that for the nonneoplastic conclusions, there are dozens of neoplastic and nonneoplastic findings clearly related to DEHP. He questioned why the acute inflammation in the uterus in the perinatal/postnatal study was considered an effect. He also asked why the bone marrow in females in the postweaning-only study was included as a finding and if it was possibly a false positive.

- Dr. Blystone said that although the response was not strong, NTP considered the bone marrow lesion exposure related since it was observed in males and females. The acute inflammation in the uterus was considered exposure related. NTP can review this and clarify.

○ Dr. Susan Elmore stated that she can see Dr. Elwell's point about the acute inflammation of the uterus possibly not being related to exposure, but that this was something NTP would need to discuss further.

\section{G.2.5.3.4. Panel Discussion}

Dr. Tracie Bunton said she wanted to see the presentation of the benchmark dose analysis, but beyond that, just had minor edits submitted in writing.

Dr. Spade asked if Dr. Elwell's comments about bone marrow and uterus related to one of the conclusions on which the panel would vote. He wondered if it was about including it in the abstract rather than the conclusion that there was a finding.

- Dr. Elwell said while the other findings listed in the abstract were convincing effects, the rationale to include the non-dose-related difference of acute uterus inflammation in the perinatal-post weaning study was not clear although there may have been reason to include it as chronic uterus inflammation was increased in the postweaningonly study. The question on including the bone marrow finding in females from the postweaning-only study in the abstract was based on very small group differences in incidence with no apparent effect on severity.

Dr. Bunton noted that a number of genetic toxicity tests were conducted and asked if they were related to this particular report or conducted over time.

- Dr. Blystone said they were not related to this report itself. They were accumulated over time and not published previously, so they were published with this report. 


\section{G.2.5.4. Vote on NTP Conclusions}

\section{G.2.5.4.1. Male Hsd:Sprague Dawley ${ }^{\circledR} S D^{\circledR}$ rats (perinatal and postweaning feed study)}

Dr. Ludewig called for a motion from the panel to approve the conclusions as written. Dr. Bunton so moved, and Dr. Wise seconded the motion. The panel voted unanimously ( 5 yes, 0 no, 0 abstentions) to approve the conclusions as written.

\section{G.2.5.4.2. Female Hsd:Sprague Dawley ${ }^{\circledR} S D^{\circledR}$ rats (perinatal and postweaning feed study)}

Dr. Ludewig called for a motion from the panel to approve the conclusions as written. Dr. Wise so moved, and Dr. Elwell seconded the motion. Dr. Elwell asked to amend the motion to vote and moved that NTP delete "and increased incidences of gross lesions of the" from the conclusion and add "and uterus." Dr. Wise motioned to accepted revisions to the conclusion and Dr. Spade seconded the motion. The panel voted unanimously ( 5 yes, 0 no, 0 abstentions) to approve the new conclusion.

In a second round of revisions, Dr. Blystone noted that the end of a sentence was cut off. He added "and gross observations in the female reproductive tract" to the end of the final conclusion. Dr. Ludewig called for a motion from the panel to approve the second round of revised conclusions. Dr. Wise so moved, and Dr. Elwell seconded the motion. The panel voted unanimously ( 5 yes, 0 no, 0 abstentions) to approve the new conclusion, below.

Revised Conclusion:

- Clear evidence of carcinogenic activity

- Increased incidence of hepatocellular adenoma or carcinoma (combined).

- The occurrence of pancreatic acinar adenoma or carcinoma (combined) was considered to be related to exposure. (Some evidence)

- The occurrence of uterine (including cervix) adenoma, adenocarcinoma, squamous cell carcinoma, or squamous cell papilloma (combined) in female rats may have been related to exposure. (Equivocal evidence)

- Exposure to DEHP resulted in increased incidences of nonneoplastic lesions in the liver, and increased incidences of gross lesions of the kidney, and uterus in female rats and gross observations in the female reproductive tract.

G.2.5.4.3. Male Hsd:Sprague Dawley ${ }^{\circledR} S D^{\circledR}$ rats (postweaning-only study)

Dr. Ludewig called for a motion from the panel to approve the conclusions as written. Dr. Wise so moved, and Dr. Elwell seconded the motion. The panel voted unanimously ( 5 yes, 0 no, 0 abstentions) to approve the conclusions as written.

\section{G.2.5.4.4. Female Hsd:Sprague Dawley ${ }^{\circledR} S D^{\circledR}$ rats (postweaning-only study)}

Dr. Ludewig called for a motion from the panel to approve the conclusions as written. The panel did not offer a motion. Dr. Elwell moved that NTP delete the reference to increased incidences of nonneoplastic lesions in the bone marrow from the conclusion. Dr. Wise seconded the motion. The panel voted unanimously ( 5 yes, 0 no, 0 abstentions) to approve the new conclusion, below.

Revised Conclusion: 
- Clear evidence of carcinogenic activity

- Increased incidences of hepatocellular adenoma or carcinoma (combined) and uterine (including cervix) adenoma, adenocarcinoma, squamous cell carcinoma, or squamous cell papilloma (combined).

- The occurrence of pancreatic acinar adenoma or carcinoma (combined) in female rats was considered to be related to exposure. (Some evidence)

- Exposure to DEHP resulted in increased incidences of nonneoplastic lesions in the liver, pancreas, bone marrow, and uterus in female rats.

G.2.5.4.5. Hsd:Sprague Dawley ${ }^{\circledR} S D^{\circledR}$ rats (comparative benchmark dose analyses)

Dr. Ludewig called for a motion from the panel to approve the conclusions as written. Dr. Wise so moved, and Dr. Elwell seconded the motion. The panel voted unanimously (5 yes, 0 no, 0 abstentions) to approve the conclusions as written.

\section{G.2.5.5. Final Conclusions}

Because revisions were proposed and approved during the meeting, the final approved conclusions are presented below:

- Perinatal and Postweaning Feed Study:

- Clear evidence of carcinogenic activity in male Hsd:Sprague Dawley ${ }^{\circledR} \mathrm{SD}^{\circledR}$ rats based on the increased incidences of hepatocellular adenoma or carcinoma (combined) and acinar adenoma or carcinoma (combined) neoplasms (predominantly adenomas) of the pancreas.

- Exposure to DEHP resulted in increased incidences of nonneoplastic lesions in the liver, kidney, bone marrow, heart, pituitary gland, testis, and epididymis and increased incidences of gross lesions of the reproductive tract

- Clear evidence of carcinogenic activity in female Hsd:Sprague Dawley ${ }^{\circledR} \mathrm{SD}^{\circledR}$ rats based on the increased incidence of hepatocellular adenoma or carcinoma (combined).

- The occurrence of pancreatic acinar adenoma or carcinoma (combined) was considered to be related to exposure. (Some evidence)

- The occurrence of uterine (including cervix) adenoma, adenocarcinoma, squamous cell carcinoma, or squamous cell papilloma (combined) in female rats may have been related to exposure. (Equivocal evidence)

- Exposure to DEHP resulted in increased incidences of nonneoplastic lesions in the liver, kidney, and uterus in female rats and gross observations in the female reproductive tract.

- Postweaning-only Feed Study

- Clear evidence of carcinogenic activity in male Hsd:Sprague Dawley ${ }^{\circledR} \mathrm{SD}^{\circledR}$ rats based on the increased incidences of hepatocellular adenoma or carcinoma (combined) and increased incidences of acinar adenoma or carcinoma (combined) neoplasms (predominantly adenomas) of the pancreas. 
- The occurrence of testicular interstitial cell adenoma in male rats may have been related to exposure. (Equivocal evidence)

- Exposure to DEHP resulted in increased incidences of nonneoplastic lesions in the liver, pancreas, bone marrow, heart, pituitary gland, testis, and epididymis.

- Clear evidence of carcinogenic activity in female Hsd:Sprague Dawley ${ }^{\circledR} \mathrm{SD}^{\circledR}$ rats based on the increased incidences of hepatocellular adenoma or carcinoma (combined) and uterine (including cervix) adenoma, adenocarcinoma, squamous cell carcinoma, or squamous cell papilloma (combined).

- The occurrence of pancreatic acinar adenoma or carcinoma (combined) in female rats was considered to be related to exposure. (Some evidence)

- Exposure to DEHP resulted in increased incidences of nonneoplastic lesions in the liver, pancreas, and uterus in female rats.

- Comparative Carcinogenic Benchmark Dose Analyses

$\circ$ No consistent pattern indicating that perinatal and postweaning exposure was more sensitive compared to postweaning-only exposure and modeled responses were within threefold of each other.

- However, there was a stronger carcinogenic response in the reproductive organs (uterus and testis) in the postweaning-only exposure study compared to the perinatal and postweaning exposure study.

\section{G.2.6. Closing Remarks on the Draft Reports}

Dr. Gabriele Ludewig welcomed additional panel comments on the draft report. There were no additional comments.

Closing the meeting, Dr. Sheena Scruggs thanked all the peer-review panelists.

Dr. Ludewig added her thanks to the NTP staff and the panel members for their efforts.

Dr. Ludewig adjourned the meeting at 2:00 p.m. EDT on April 2, 2021. 


\section{Appendix H. Supplemental Data}

Tables with supplemental data can be found here: https://doi.org/10.22427/NTP-DATA-TR$601 .{ }^{202}$

\section{H.1. Perinatal and Postweaning Study in Rats (Study 1)}

\section{H.1.1. Data Tables}

E01 - Animal Removal Summary by Treatment Group

1018802_E01_Animal_Removal_Summary_By_Treatment_Group.pdf

E02 - Animals Removed from Experiment

1018802_E02_Animals_Removed_from_Experiment.pdf

E03 - Growth Curves

1018802_E03_Growth_Curves_Litter_Based.pdf

E04 - Mean Body Weights and Survival Table

1018802_E04_Mean_Body_Weights_and_Survival_Table_Litter_Based.pdf

E05 - Clinical Observations Summary

1018802_E05_Clinical_Observations_Summary.pdf

E08 - Feed Water and Compound Consumption Table

1018802_E08_Feed_Water_and_Compound_Consumption_Table.pdf

Gestational Body Weights

1018802_Gestational_Body_Weights.pdf

Gestational Food Consumption

1018802_Gestational_Food_Consumption.pdf

Gestational and Lactational Chemical Consumption

1018802_Gestational_and_Lactational_Chemical_Consumption.pdf

Lactational Body Weights

1018802_Lactational_Body_Weights.pdf

Lactational Food Consumption

1018802_Lactational_Food_Consumption.pdf

P03 - Incidence Rates of Non-Neoplastic Lesions by Anatomic Site

1018802_P03_Incidence_Rates_of_Non-Neoplastic_Lesions_by_Anatomic_Site.pdf

P04 - Neoplasms by Individual Animal

1018802_P04_Neoplasms_by_Individual_Animal.pdf

P05 - Incidence Rates of Neoplasms by Anatomic Site (Systemic Lesions Abridged)

1018802_P05_Incidence_Rates_of_Neoplasms_by_Anatomic_Site_(Systemic_Lesions_Abridge d).pdf 
P08 - Statistical Analysis of Primary Tumors

1018802_P08_Statistical_Analysis_of_Primary_Tumors.pdf

P09 - Non-Neoplastic Lesions by Individual Animal

1018802_P09_Non-Neoplastic_Lesions_by_Individual_Animal.pdf

P10 - Statistical Analysis of Non-Neoplastic Lesions - Litter based

1018802_P10_Statistical_Analysis_of_Non-Neoplastic_Lesions.pdf

P11 - Statistical Analysis of Survival Data

1018802_P11_Statistical_Analysis_of_Survival_Data.pdf

P14 - Individual Animal Pathology Data

1018802_P14_Individual_Animal_Pathology_Data.pdf

P17 - Neoplasms by Individual Animal (Systemic Lesions Abridged)

1018802_P17_Neoplasms_By_Individual_Animal_(Systemic_Lesions_Abridged).pdf

P18 - Incidence Rates of Non-Neoplastic Lesions by Anatomic Site with Average Severity Grades

1018802_P18_Incidence_Rates_of_Non-

Neoplastic_Lesions_by_Anatomic_Site_with_Average_Severity_Grades.pdf

P22 - Cause of Death Summary

1018802_Cause_of_Death_Summary.pdf

P40 - Survival Curves

1018802_P40_Survival_Curves.pdf

PA46Rs - Summary of Gross Pathology with Litter Incidence

1018802_PA46Rs_Summary_of_Gross_Pathology_with_Litter_Incidence.pdf

PA48 - Summary of Tissue Concentration

1018802_PA48_Summary_of_Tissue_Concentration.pdf

PND 1 Litter Data

1018802_PND_1_Litter_Data.pdf

PND 4 Litter Size and Survival Data

1018802_PND_4_Litter_Size_and\%20_Survival.pdf

Pup Body Weights

1018802_Pup_Body_Weights.pdf

R02 - Reproductive Performance Summary

1018802_R02_Reproductive_Performance_Summary.pdf

R23 - Gubernaculum Length Summary

1018802_R23_Gubernaculum_Length_Summary.pdf 


\section{H.1.2. Individual Animal Data}

Female Individual Animal Body Weight Data All Animals

1018802_Female_Individual_Animal_Body_Weight_Data_All_Animals.xls

Female Individual Animal Body Weight Data All Animals - Lactation 1018898_Female_Individual_Animal_Body_Weight_Data_All_Animals.xls

Female Individual Animal Body Weight Data All Animals - Gestation 1018899_Female_Individual_Animal_Body_Weight_Data_All_Animals.xls

Female Individual Animal Clinical Observations

1018802_Female_Individual_Animal_Clinical_Observations.xls

Female Individual Animal Neoplastic Pathology Data

1018802_Female_Individual_Animal_Neoplastic_Pathology_Data.xls

Female Individual Animal Non-Neoplastic Pathology Data

1018802_Female_Individual_Animal_Nonneoplastic_Pathology_Data.xls

Female Individual Animal Survival Data

1018802_Female_Individual_Animal_Survival_Data.xls

Female Individual Animal Survival Data - Gestation

1018899_Female_Individual_Animal_Survival_Data.xls

Female Individual Animal Survival Data - Lactation

1018898_Female_Individual_Animal_Survival_Data.xls

Female Individual Animal Terminal Body Weight Data

1018802_Female_Individual_Animal_Terminal_Body_Weight_Data.xls

Female Individual Animal Terminal Body Weight Data - Gestation

1018899_Female_Individual_Animal_Terminal_Body_Weight_Data.xls

Female Individual Animal Terminal Body Weight Data - Lactation

1018898_Female_Individual_Animal_Terminal_Body_Weight_Data.xls

Male Individual Animal Body Weight Data All Animals

1018802_Male_Individual_Animal_Body_Weight_Data_All_Animals.xls

Male Individual Animal Clinical Observations

1018802_Male_Individual_Animal_Clinical_Observations.xls

Male Individual Animal Neoplastic Pathology Data

1018802_Male_Individual_Animal_Neoplastic_Pathology_Data.xls

Male Individual Animal Non-Neoplastic Pathology Data

1018802_Male_Individual_Animal_Nonneoplastic_Pathology_Data.xls

Male Individual Animal Survival Data

1018802_Male_Individual_Animal_Survival_Data.xls 
Male Individual Animal Terminal Body Weight Data 1018802_Male_Individual_Animal_Terminal_Body_Weight_Data.xls

Individual Animal Clinical Observations Data 1018802_Individual_Animal_Clinical_Observations_Data.xlsx

Individual Animal DamID and PupID Data

1018802_Individual_Animal_DamID_and_PupID_Data.xlsx

Individual Animal Reproductive Performance Data

1018802_Individual_Animal_Reproductive_Performance_Data.xlsx

Individual Animal Tissue Concentration Data

1018802_Individual_Animal_Tissue_Concentration_Data.xlsx

Individual Pup Census and Litter Weight by Sex Data

1018802_Individual_Pup_Census_and_Litter_Weight_by_Sex_Data.xlsx

Gubernaculum and Urogenital Findings Data

1018802 Gubernaculum and_Urogenital_Findings_Data.xls

\section{H.2. Postweaning-only Study in Rats (Study 2)}

\section{H.2.1. Data Tables}

E01 - Animal Removal Summary by Treatment Group

1018801_E01_Animal_Removal_Summary_By_Treatment_Group.pdf

E02 - Animals Removed from Experiment

1018801_E02_Animals_Removed_from_Experiment.pdf

E03 - Growth Curves

1018801_E03_Growth_Curves.pdf

E04 - Mean Body Weights and Survival Table

1018801_E04_Mean_Body_Weights_and_Survival_Table.pdf

E05 - Clinical Observations Summary

1018801_E05_Clinical_Observations_Summary.pdf

E08 - Feed Water and Compound Consumption Table

1018801_E08_Feed_Water_and_Compound_Consumption_Table.pdf

P03 - Incidence Rates of Non-Neoplastic Lesions by Anatomic Site

1018801_P03_Incidence_Rates_of_Non-Neoplastic_Lesions_by_Anatomic_Site.pdf

P04 - Neoplasms by Individual Animal

1018801_P04_Neoplasms_by_Individual_Animal.pdf

P05 - Incidence Rates of Neoplasms by Anatomic Site (Systemic Lesions Abridged)

1018801_P05_Incidence_Rates_of_Neoplasms_by_Anatomic_Site_(Systemic_Lesions_Abridge d).pdf 
P08 - Statistical Analysis of Primary Tumors

1018801_P08_Statistical_Analysis_of_Primary_Tumors.pdf

P09 - Non-Neoplastic Lesions by Individual Animal

1018801_P09_Non-Neoplastic_Lesions_by_Individual_Animal.pdf

P10 - Statistical Analysis of Non-Neoplastic Lesions

1018801_P10_Statistical_Analysis_of_Non-Neoplastic_Lesions.pdf

P11 - Statistical Analysis of Survival Data

1018801_P11_Statistical_Analysis_of_Survival_Data.pdf

P14 - Individual Animal Pathology Data

1018801_P14_Individual_Animal_Pathology_Data.pdf

P17 - Neoplasms by Individual Animal (Systemic Lesions Abridged)

1018801_P17_Neoplasms_By_Individual_Animal_(Systemic_Lesions_Abridged).pdf

P18 - Incidence Rates of Non-Neoplastic Lesions by Anatomic Site with Average Severity Grades

1018801_P18_Incidence_Rates_of_Non-

Neoplastic_Lesions_by_Anatomic_Site_with_Average_Severity_Grades.pdf

P22 - Cause of Death Summary

1018801_P22_Cause_of_Death_Summary

P40 - Survival Curves

1018801_P40_Survival_Curves.pdf

\section{H.2.2. Individual Animal Data}

Female Individual Animal Body Weight Data All Animals

1018801_Female_Individual_Animal_Body_Weight_Data_All_Animals.xls

Female Individual Animal Clinical Observations

1018801_Female_Individual_Animal_Clinical_Observations.xls

Female Individual Animal Neoplastic Pathology Data

1018801_Female_Individual_Animal_Neoplastic_Pathology_Data.xls

Female Individual Animal Non-Neoplastic Pathology Data

1018801_Female_Individual_Animal_Non_Neoplastic_Pathology_Data.xls

Female Individual Animal Survival Data

1018801_Female_Individual_Animal_Survival_Data.xls

Female Individual Animal Terminal Body Weight Data

1018801_Female_Individual_Animal_Terminal_Body_Weight_Data.xls

Male Individual Animal Body Weight Data All Animals

1018801_Male_Individual_Animal_Body_Weight_Data_All_Animals.xls 
Male Individual Animal Clinical Observations

1018801_Male_Individual_Animal_Clinical_Observations.xls

Male Individual Animal Neoplastic Pathology Data

1018801_Male_Individual_Animal_Neoplastic_Pathology_Data.xls

Male Individual Animal Non-Neoplastic Pathology Data

1018801_Male_Individual_Animal_Non_Neoplastic_Pathology_Data.xls

Male Individual Animal Survival Data

1018801_Male_Individual_Animal_Survival_Data.xls

Male Individual Animal Terminal Body Weight Data

1018801_Male_Individual_Animal_Terminal_Body_Weight_Data.xls

\section{H.3. Benchmark Dose Analysis}

\section{H.3.1. Results Summary}

Summary of BMD Results

BMD_Results_Summary.xlsx

\section{H.3.2. Perinatal and Postweaning Study in Rats (Study 1)}

Perinatal Female Liver BMD Analysis

Perinatal_Female_LiverBMD_analysis.xlsx

Perinatal Female Pancreas BMD Analysis

Perinatal_Female_Pancreas_BMD_analysis.xlsx

Perinatal Female Uterus BMD Analysis

Perinatal_Female_Uterus_BMD_analysis.xlsx

Perinatal Male Liver BMD Analysis

Perinatal_Male_Liver_BMD_analysis.xlsx

Perinatal Male Pancreas BMD Analysis

Perinatal_Male_Pancreas_BMD_analysis.xlsx

Perinatal Male Testes BMD Analysis

Perinatal_Male_Testes_BMD_analysis.xlsx

\section{H.3.3. Postweaning-only Study in Rats (Study 2)}

Adult Female Liver BMD Analysis

Adult_Female_Liver_BMD_analysis.xlsx

Adult Female Pancreas BMD Analysis

Adult_Female_Pancreas_BMD_analysis.xlsx

Adult Female Uterus BMD Analysis

Adult_Female_Uterus_BMD_analysis.xlsx 
Adult Male Liver BMD Analysis

Adult_Male_Liver_BMD_analysis.xlsx

Adult Male Pancreas BMD Analysis

Adult_Male_Pancreas_BMD_analysis.xlsx

Adult Male Testes BMD Analysis

Adult_Male_Testes_BMD_analysis.xlsx

\section{H.4. Genetic Toxicology}

\section{H.4.1. In Vivo Peripheral Blood Micronucleus Study A04384 (Dosed Feed)}

G04 - In Vivo Micronucleus Summary Data

A04384_G04_In_Vivo_Micronucleus_Summary_Data.pdf

Individual Animal In Vivo Micronucleus Data

A04384_Individual_Animal_In_Vivo_Micronucleus_Data.xlsx

\section{H.4.2. In Vivo Peripheral Blood Micronucleus Study A15851 (Dermal)}

G04 - In Vivo Micronucleus Summary Data

A15851_G04_In_Vivo_Micronucleus_Summary_Data.pdf

Individual Animal In Vivo Micronucleus Data

A15851_Individual_Animal_In_Vivo_Micronucleus_Data.xlsx

\section{H.4.3. Rodent Chromosome Aberrations Study A15927 (Dosed Feed)}

Data can be found here: https://manticore.niehs.nih.gov/cebssearch/genetox/002-01969-0036$\underline{0000-6 /}$

\section{H.4.4. In Vivo Bone Marrow Micronucleus Study A15927 (Dosed Feed)}

G04 - In Vivo Micronucleus Summary Data

A15927_G04_In_Vivo_Micronucleus_Summary_Data.pdf

Individual Animal In Vivo Micronucleus Data

A15927_Individual_Animal_In_Vivo_Micronucleus_Data.xlsx 


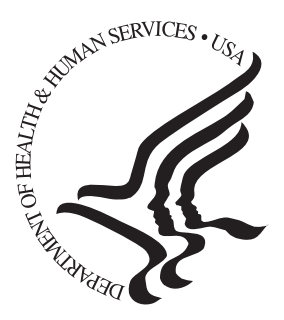

National Toxicology Program

National Institute of Environmental Health Sciences

National Institutes of Health

P.O. Box 12233, MD K2-05

Durham, NC 27709

Tel: 984-287-3211

ntpwebrequest@niehs.nih.gov 